\title{
Topics on Transient Dirichlet Forms: Fundamental Solutions, Potential Kernels and Metric Measure Spaces
}

\author{
Huw Thomas Fry \\ Submitted to Swansea University in fulfilment of the \\ requirements for the Degree of Doctor of Philosophy
}

Department of Mathematics

College of Science

Swansea University

2020 
(c) The copyright of this thesis belongs to the author under the terms of the United Kingdom Copyright Acts as qualified by the Swansea University Regulation 3.49. Due acknowledgment must always be made of the use of any material contained in, or derived from, this thesis. 


\section{DECLARATION}

This work has not previously been accepted in substance for any degree and is not being concurrently submitted in candidature for any degree.

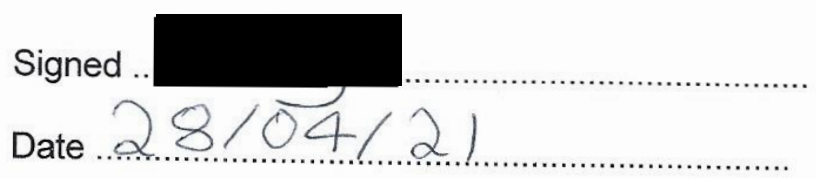

\section{STATEMENT 1}

This thesis is the result of my own investigations, except where otherwise stated. Where correction services have been used, the extent and nature of the correction is clearly marked in a footnote(s).

Other sources are acknowledged by footnotes giving explicit references. A bibliography is appended.

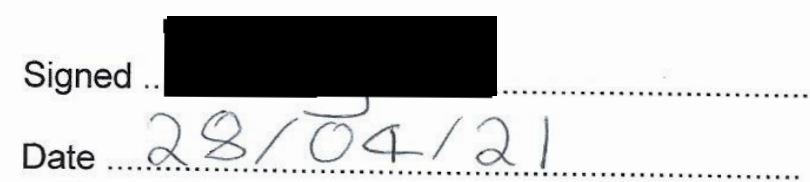

\section{STATEMENT 2}

I hereby give consent for my thesis, if accepted, to be available for photocopying and for inter-library loan, and for the title and summary to be made available to outside organisations.

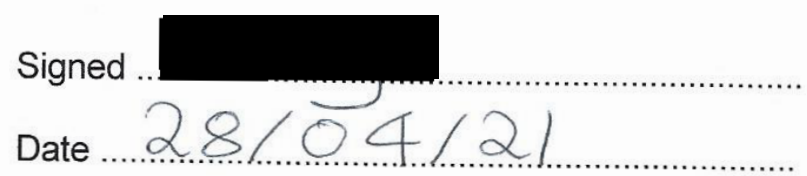




\section{Acknowledgements}

I would like to take this opportunity to thank my supervisor Prof. Neils Jacob for the discussions and proposals throughout the years and the time taken to read through my manuscripts. I would also like to thank all the staff in the department for their encouragement throughout the course of my research. To my examiners, Prof. Ian Davies and Dr. Alexei Daletskii I also owe thanks for the time taken to review my work.

A special acknowledgement goes to my former teachers: Michael Boulding and Steven Hamlyn for their continued help and guidance during the early stages of my academic life.

A heartfelt thank you goes to my family for all their encouragement, support and understanding during the last years.

Finally, I would like to thank the Swansea University Doctorial Training Centre at the College of Science and the EPSRC for their support in funding this work, without which this would not have been possible. 


\section{Abstract}

In this thesis we study the pseudo-differential operator $-\psi(D)$ where on $\mathcal{S}\left(\mathbb{R}^{n}\right)$ we have $\psi(D) u(x)=(2 \pi)^{-\frac{n}{2}} \int_{\mathbb{R}^{n}} e^{i x \xi} \psi(\xi) \hat{u}(\xi) d \xi$ with a continuous negative definite function $\psi: \mathbb{R}^{n} \rightarrow \mathbb{R}$ as the symbol. We find conditions for the existence of a fundamental solution to $\psi(D)$ which is given by $E=(2 \pi)^{-\frac{n}{2}} F^{-1}(\underline{1})$ where with the help of the scale of anisotropic Bessel potential spaces $H^{\psi, s}\left(\mathbb{R}^{n}\right), s \in \mathbb{R}$, we obtain $E \in H^{\psi,-\frac{s_{0}}{2}}\left(\mathbb{R}^{n}\right)$ for suitable $s_{0}$. This allows us to study regularity properties of $u:=E * f, f \in H^{\psi, t}\left(\mathbb{R}^{n}\right)$, which solves $\psi(D) u=f$.

We find that $d_{\psi}(\xi, \eta):=\frac{1}{2}(\xi-\eta)$ is a metric on $\mathbb{R}^{n}$ which arises from a continuous negative definite function $\psi: \mathbb{R}^{n} \rightarrow \mathbb{R}$ which satisfies $\psi(\xi)=0$ if, and only if, $\xi=0$. Given additional properties we show that the corresponding Dirichlet form $\left(\mathcal{E}^{\psi}, H^{\psi, 1}\left(\mathbb{R}^{n}\right)\right)$ is transient and its potential kernel $\kappa$ is given by $F^{-1}\left(\frac{1}{\psi}\right)$. We evaluate that the fundamental solution and the potential kernel coincide where the potential kernel is up to a constant equivalent to the fundamental solution of $\psi(D)$.

Finally, we discuss the fundamental solution in relation with the metric $d_{\psi}(\xi, \eta)=$ $\frac{1}{2}(\xi-\eta)$. We find in particular that the fundamental solution which is rotational invariant can be described with the help of $d_{\psi}(\xi-\eta)=g\left(\|\xi-\eta\|^{2}\right)$. Moreover, we obtain some further results in which the density of $\kappa$ is a function of $d_{\psi}$. 


\section{Contents}

$\begin{array}{ll}\text { Introduction } & 1\end{array}$

1 Fourier Analysis $\quad 7$

1.1 Fourier Transform . . . . . . . . . . . . . . . . . 7

1.2 Negative Definite Functions and Convolution Semigroups . . . . 14

1.3 Lévy-Khinchin Formula . . . . . . . . . . . . . . . . . . . . . 18

1.4 Bernstein Functions and Subordination . . . . . . . . . . . . 20

2 Semigroups and Their Generators 25

2.1 Results from Functional Analysis . . . . . . . . . . . . . . 25

2.2 One-Parameter Semigroups . . . . . . . . . . . . . . 32

2.3 Dirichlet Spaces . . . . . . . . . . . . . . . . . . 35

2.4 Pseudo-Differential Operators with Negative Definite Symbols . 40

3 Metric Measure Spaces $\quad 55$

3.1 Transition Densities and Metric Measure Space . . . . . . . . 55

3.2 Examples Involving some Transition Densities . . . . . . . . . . 58

4 Distributions in the Sense of L.Schwartz and Fundamental So- 
$\begin{array}{ll}\text { lutions } & 61\end{array}$

4.1 Distributions ....................... 61

4.2 Fundamental Solutions for Partial Differential Operators with Constant Coefficients . . . . . . . . . . . . 66

4.3 The Spaces $H^{\psi, s}\left(\mathbb{R}^{n}\right)$ and Related Spaces . . . . . . . . . . . 72

4.4 On Fundamental Solutions of Pseudo-Differential Operators with Negative Definite Symbols . . . . . . . . . . . . 80

5 Transient Semigroups, Potential Kernels and Fundamental So$\begin{array}{lr}\text { lutions } & 89\end{array}$

5.1 Translation Invariant Extended Dirichlet Spaces ........................... 90

5.2 Transient Convolution Semigroups . . . . . . . . . . . . . . . 97

5.3 Fundamental Solutions as Potential Kernels . . . . . . . . . . . 102

5.4 Comparison Results for Extended Dirichlet Spaces . . . . . . . . 105

6 Potential Kernels and Intrinsic Metrics 113

6.1 Some First Properties of Potential Kernels . . . . . . . . . . . . 113

6.2 Examples ....................... 116

6.3 Interpretations and Generalization . . . . . . . . . . . . 132

$\begin{array}{ll}\text { Bibliography } & 137\end{array}$ 


\section{Notation}

$\mathbb{N}$ natural numbers

$\mathbb{N}_{0}=\mathbb{N} \cup\{0\}$

$\mathbb{N}_{0}^{n}$ set of all multiindices

$\mathbb{R}$ real numbers

$\mathbb{R}^{n}$ Euclidean vector space

$\mathbb{C}$ complex numbers

$a \wedge b=\min (a, b)$

$a \vee b=\max (a, b)$

$u^{+}=u \vee 0$ positive part of $u$

$u^{-}=(-u \vee 0)$ negative part of $u$

$\bar{A}$ closure of a set

$\mathcal{B}^{(n)}$ Borel $\sigma$-algebra on $\mathbb{R}^{n}$

$\mathcal{B}(G)$ Borel sets of a topological space $G$

Re $f$ real part of a function

Im $f$ imaginary part of a function

$\left(u_{\nu}\right)_{\nu \in \mathbb{N}}$ sequence of functions $u_{\nu}$

$f \circ g$ composition of functions

$u * v$ convolution of functions

$S x=-x$ reflection at the origin

$T_{a} x=x+a$ translation by $a$

$F u$ Fourier transform of $u$

$F^{-1} u$ inverse Fourier transform of $u$ 
$\mathcal{L} u$ Laplace transform of $u$

$\lambda^{(n)}$ Lebesgue measure in $\mathbb{R}^{n}$

$\varepsilon_{a}$ Dirac measure at $a \in \mathbb{R}^{n}$

$|\alpha|=\alpha_{1}+\ldots+\alpha_{n}$ for $\alpha \in \mathbb{N}_{0}^{n}$

$\partial^{\alpha} u=\frac{\partial^{|\alpha|} u}{\partial x_{1}^{\alpha_{1} \ldots \partial x_{n}^{\alpha_{n}}}}$

$\mu_{1} \otimes \mu_{2}$ product of the measures $\mu_{1}$ and $\mu_{2}$

$\mu_{1} * \mu_{2}$ convolution of the measures $\mu_{1}$ and $\mu_{2}$

$\|u\|$ total mass of a measure $\mu$

supp $\mu$ support of a measure $\mu$

$\left(\mu_{t}\right)_{t \geq 0}$ convolution semigroup of subprobabilites

$\left(\mu_{t}^{f}\right)_{t \geq 0}$ subordinate convolution semigroup

$(X, d)$ metric space with metric $d(\cdot, \cdot)$ on $X \times X$

$(X, d, \mu)$ metric measure space with metric $d$ and measure $\mu$

$C(G)$ continuous functions

$C_{0}(G)$ continuous functions with compact support

$C_{\infty}(G)$ continuous functions vanishing at infinity

$C_{b}(G)$ bounded continuous functions

$C^{m}(G)$ m-times continuously differentiable functions

$C_{0}^{m}(G)=C^{m}(G) \cap C_{0}(G)$

$C^{\infty}(G)=\bigcap_{m \in \mathbb{N}} C^{m}(G)$

$C_{0}^{\infty}(G)=\bigcap_{m \in \mathbb{N}} C_{0}^{m}(G)$

$\mathscr{D}^{\prime}(G)$ vector space of distributions on $G$

$\mathcal{E}^{\prime}(G)$ space of all distributions with compact support

$L^{p}(\Omega, \mu)$ usual Lebesgue space over $(\Omega, \mathcal{A}, \mu)$

$L^{p}(G)=L^{p}\left(G, \lambda^{(n)}\right)$

$H^{\psi, s}\left(\mathbb{R}^{n}\right)=\left\{u \in \mathcal{S}^{\prime}\left(\mathbb{R}^{n}\right) ;\|u\|_{\psi, s}<\infty\right\}$

$\mathcal{M}_{b}^{+}(\Omega)$ bounded measures on $\Omega$

$\mathcal{S}\left(\mathbb{R}^{n}\right)$ Schwartz space of tempered functions

$\mathcal{S}^{\prime}\left(\mathbb{R}^{n}\right)$ tempered distributions

$\mathcal{S}$ Stieltjes functions 
$C N\left(\mathbb{R}^{n}\right)$ continuous negative definite functions

$N\left(\mathbb{R}^{n}\right)$ negative definite functions

MCN continuous negative definite functions that induce a metric on $\mathbb{R}^{n}$ which generates the Euclidean topology

$\|u\|_{X}$ norm of $u$ in the space $X$

$\|u\|_{0}$ norm in $L^{2}(\Omega, \mu)$

$\|u\|_{A}=\|u\|_{X}+\|A u\|_{X}$ graph norm of $A$

$\|u\|_{\infty}=\sup |u(x)|$ or $\operatorname{esssup}|u(x)|$

$\|u\|_{s}$ norm in the space $H^{s}\left(\mathbb{R}^{n}\right)$

$\|u\|_{\psi, s}$ norm in the space $H^{\psi, s}\left(\mathbb{R}^{n}\right)$

$(X,\|\cdot\|)_{X}$ Banach space with norm $\|\cdot\|_{X}$

$X \hookrightarrow Y$ continuous embedding of $X$ into $Y$

$\langle u, x\rangle$ duality pairing between $X^{*}$ and $X$

$p_{\alpha, \beta}(u)=\sup _{x \in \mathbb{R}^{n}}\left|x^{\beta} \partial^{\alpha} u(x)\right|$

$(A, D(A))$ linear operator with domain $D(A)$

$D(A)$ domain of an operator

$\Gamma(A)$ graph of an operator

$\bar{A}$ closure of an operator

$A^{*}$ conjugate operator or adjoint Hilbert space operator

$\rho(A)$ resolvent set of an operator

$R_{\lambda}=(\lambda-A)^{-1}$ resolvent of operator $A$

$B_{\lambda}(u, v)=B(u, v)+\lambda(u, v)_{L^{2}}$ for a bilinear form $B$

$q(x, D)$ pseudo-differential operator with symbol $q(x, \xi)$

$\psi(D)$ pseudo-differential operator with symbol $\psi(\xi)$

$|x|$ Euclidean distance in $\mathbb{R}^{n}$

$\mathcal{E}(\cdot, \cdot)$ Dirichlet form

$\left(T_{t}\right)_{t \geq 0}$ one parameter semigroup of operators

$\left(T_{t}^{(\infty)}\right)_{t \geq 0}$ semigroup on $C_{\infty}\left(\mathbb{R}^{n}\right)$

$A^{(\infty)}$ generator of $\left(T_{t}^{(\infty)}\right)_{t \geq 0}$

$\left(T_{t}^{(p)}\right)_{t \geq 0}$ semigroup on $L^{p}\left(\mathbb{R}^{n}\right), 1<p<\infty$

$A^{(p)}$ generator of $\left(T_{t}^{(p)}\right)_{t \geq 0}$ 


\section{Introduction}

We can study the Laplace operator $\Delta_{n}$ from many different points of view. These include as a classical second order partial differential operator, along with the starting point for investigating the Newton potential. Moreover they may include as an operator related to the Euler-Lagrange equation for a certain quadratic problem in the calculus of variations, hence as generator of a Dirichlet form, as well as generator of the Brownian semigroup, hence as generator of a diffusion process, and also as the Laplace-Beltrami-operator in flat space with corresponding Riemannian metric being the Euclidean as linear partial operator acting on distributions in the sense of Laurent Schwartz, etc. Of course there are plenty of relations of the results studied in each of these topics, indeed the richness of the theory of the Laplacian is due to these many connections. It is natural to ask which of the results valid for the Laplace operator can be transferred to other operator classes, and to which extent can we recover or interpret the relation of these results along the similar way we can for the Laplacian.

In this thesis we want to study generators of symmetric $L^{2}$ sub-Markovian semigroups on $L^{2}\left(\mathbb{R}^{n}\right)$ which are pseudo-differential operators $-\psi(D)$ where on $\mathcal{S}\left(\mathbb{R}^{n}\right)$ we have

$$
\psi(D) u(x)=(2 \pi)^{-\frac{n}{2}} \int_{\mathbb{R}^{n}} e^{i x \xi} \psi(\xi) u(\xi) d \xi
$$

with a continuous negative definite function $\psi: \mathbb{R}^{n} \rightarrow \mathbb{R}$ as the symbol. If we look at $-\psi(D)$ as a pseudo-differential operator with constant coefficients we may ask for the existence of a fundamental solution for $\psi(D)$. Moreover if 
we look at $-\psi(D)$ as generator of a translation invariant Dirichlet form or as generator of an $L^{2}$ sub-Markovian semigroup we may ask whether it allows an abstract potential operator which admits a potential kernel. We may now ask how this potential kernel is related to a fundamental solution for $\psi(D)$. In the case of the Laplacian for $n \geq 3$ this leads of course to the Newton kernel which is (with a suitable normalization) the potential kernel and a fundamental solution. We may move a step further and ask whether, as in the case of the Laplacian, the symbol $\psi(\xi)$ of $\psi(D)$ has the property that $d_{\psi}(\xi, \eta):=\psi^{\frac{1}{2}}(\xi-\eta)$ defines a metric on $\mathbb{R}^{n}$ and whether the potential kernel (or the fundamental solution) can be better understood with the help of this metric. The latter question relates to the investigations started in [23] where the densities of the transition function of the Lévy process associated with the operator $-\psi(D)$ under considerations were studied with the help of the metric $d_{\psi}$.

This programme does not work out without additional assumptions and indeed some technical difficulties lead us to some severe restrictions. Nonetheless, for some non-trivial larger classes of symbols we find that the suggested programme is a reasonable one leading to interesting results and examples. On the other hand, we are left with several open questions, partly due to technical difficulties with some of a fundamental nature.

Assume that $\psi: \mathbb{R}^{n} \rightarrow \mathbb{R}$ is a continuous negative definite function satisfying the following properties

i) $\psi(\xi)=0$ if and only if $\xi=0$;

ii) $\psi(\xi) \geq c_{0}|\xi|^{\rho_{0}},|\xi| \geq R_{0}, \rho_{0}>0, c_{0}>0, R_{0} \geq 0$;

iii) $\frac{1}{\psi} \in L_{l o c}^{2}\left(\mathbb{R}^{n}\right)$;

iv) $\psi(\xi) \leq c_{1}|\xi|^{\rho_{1}}$ for $|\xi| \geq R_{1}, c_{1} \geq 0,0 \leq \rho_{1} \leq 2, R_{1} \geq 0$,

where $R_{0}$ and $R_{1}$ are independent of each other. Note that not all these conditions are required for all results throughout the thesis. However they are all 
needed in order to prove various results. In particular the first condition implies that $d_{\psi}$ is a metric on $\mathbb{R}^{n}$ which induces the Euclidean topology. Further the conditions imply that the corresponding Dirichlet form $\left(\mathcal{E}^{\psi}, H^{\psi, 1}\left(\mathbb{R}^{n}\right)\right)$ is transient and that its potential kernel $\kappa$ is given by

$$
F^{-1}\left(\frac{1}{\psi}\right) \text {, }
$$

where $\hat{u}=F u$ denotes the Fourier transform of $u$ and $F^{-1} u$ its inverse.

Moreover, the associated operator semigroup $\left(T_{t}^{\psi}\right)_{t \geq 0}$ has densities

$$
p_{t}(x)=(2 \pi)^{-n} \int_{\mathbb{R}^{n}} e^{i x \xi} e^{-t \psi(\xi)} d \xi, t>0,
$$

with $p_{t} \in L^{1}\left(\mathbb{R}^{n}\right) \cap C_{\infty}\left(\mathbb{R}^{n}\right)$. Note that for all these results $\frac{1}{\psi} \in L_{l o c}^{1}\left(\mathbb{R}^{n}\right)$ would be sufficient. In fact, see [14], $\frac{1}{\psi} \in L_{l o c}^{1}\left(\mathbb{R}^{n}\right)$ would allow us to introduce the generalized Laplacian associated with $\psi$ which can be viewed as a convolution operator on certain classes of distributions, we refer again to [14] and the reference given there.

However, since $\psi$ is in general only continuous we have some problems to treat $\psi(D)$ as an operator on all $\mathscr{D}^{\prime}\left(\mathbb{R}^{n}\right)$ or $\mathcal{S}^{\prime}\left(\mathbb{R}^{n}\right)$. While for $u \in \mathcal{S}\left(\mathbb{R}^{n}\right)$ we can define

$$
\psi(D) u=F^{-1}(\psi \hat{u})
$$

the natural idea of extending $\psi(D)$ by duality to $\mathcal{S}^{\prime}\left(\mathbb{R}^{n}\right)$ (or even to $\mathscr{D}^{\prime}\left(\mathbb{R}^{n}\right)$ ) by

$$
\langle\psi(D) u, \varphi\rangle=\langle u, \psi(D) \varphi\rangle, \varphi \in \mathcal{S}\left(\mathbb{R}^{n}\right)
$$

causes some difficulties. Clearly, since $\psi$ is by assumption real-valued, we expect (1) to hold for all $u, \varphi \in \mathcal{S}\left(\mathbb{R}^{n}\right)$, but for $\varphi \in \mathcal{S}\left(\mathbb{R}^{n}\right)$ it does in general not follow that $\psi(D) \varphi \in \mathcal{S}\left(\mathbb{R}^{n}\right)$ nor does $\psi(D) \varphi$ have a compact support if $\varphi$ has. Thus for $u \in \mathcal{S}^{\prime}\left(\mathbb{R}^{n}\right)\left(\right.$ or $\left.u \in \mathscr{D}^{\prime}\left(\mathbb{R}^{n}\right)\right)$ the term

$$
\langle u, \psi(D) \varphi\rangle, \varphi \in \mathcal{S}\left(\mathbb{R}^{n}\right)
$$

is in general not defined. This observation is critical when searching for a fundamental solution to $\psi(D)$, i.e. a distribution $E$ with the property that

$$
\psi(D) E=\varepsilon_{0}
$$


where $\varepsilon_{0}$ is the Dirac measure at 0 . More precisely, we are searching for a fundamental solution which allows us to deduce regularity properties for $E * f$ in terms of function spaces being determined by $\psi$. This emphasis on regularity properties of solutions $E * f$ of $\psi(D)=f$ in terms of such function spaces goes much further than the observation made in [14]. Once $\psi(D) E$ is properly defined and we can prove that

$$
F(\psi(D) E)=\psi(\xi) F(E)
$$

then it follows that

$$
E=(2 \pi)^{-\frac{n}{2}} F^{-1}\left(\frac{1}{\psi}\right)
$$

and that $E$ can be identified with the potential kernel. Since $\frac{1}{\psi} \in L_{l o c}^{1}\left(\mathbb{R}^{n}\right)$ implies that $\frac{1}{\psi}$ is a positive definite distribution, the Bochner-Schwartz theorem yields already the existence of $F^{-1}\left(\frac{1}{\psi}\right)$ in this case. Moreover as a consequence of the Bochner-Schwartz theorem the potential kernel which is the Fourier transform of a positive definite measure is also a positive definite distribution. However, we aim for more information on $E$ and this requires more conditions on $\frac{1}{\psi}$. The condition we add is assumption $i i i)$, i.e. $\frac{1}{\psi} \in L_{l o c}^{2}\left(\mathbb{R}^{n}\right)$. Now we can use the scale of anisotropic Bessel potential spaces $H^{\psi, s}\left(\mathbb{R}^{n}\right), s \in \mathbb{R}$, and for $s_{0}>\frac{n}{2 \rho_{0}}$ we can prove that $E \in H^{\psi,-\frac{s_{0}}{2}}\left(\mathbb{R}^{n}\right) \subset \mathcal{S}^{\prime}\left(\mathbb{R}^{n}\right)$ which will act on certain function spaces $H^{\psi, t}\left(\mathbb{R}^{n}\right)$ by convolution allowing to study $u:=E * f, f \in H^{\psi, t}\left(\mathbb{R}^{n}\right)$. We may now apply regularity results such as

$$
\operatorname{sing} \operatorname{supp} u \subset \operatorname{sing} \operatorname{supp} E+\operatorname{sing} \operatorname{supp} f
$$

which have their roots in the theory of distributions. But we can also use the fact that $E$ which is equivalent to $\kappa$ is a non-negative potential kernel to derive further properties of $u$. Note that $\kappa$ is of at most polynomial growth and has a density with respect to $\lambda^{(n)}$ which however is in general not integrable over $\mathbb{R}^{n}$. Note that we can relax the condition $\frac{1}{\psi} \in L_{l o c}^{2}\left(\mathbb{R}^{n}\right)$ to $\frac{1}{\psi} \in L_{l o c}^{p}\left(\mathbb{R}^{n}\right)$ for some $1<p$. This requires non-trivial analysis in the frame of $\psi$-Bessel potential spaces $H_{p}^{\psi, s}\left(\mathbb{R}^{n}\right)$ as introduced in [9] and this is worked out in [8].

Next we consider the fact that $d_{\psi}(\xi, \eta)=\psi^{\frac{1}{2}}(\xi-\eta)$ is a metric on $\mathbb{R}^{n}$ and we 
ask whether the potential kernel $\kappa$ (which is up to a constant the fundamental solution to $\psi(D))$ can be studied with the help of $d_{\psi}$. For example we may look at regularity results for $E * h$ in relation to Hölder-conditions imposed on $h$ in terms of $d_{\psi}$. If for example $\psi$ is rotational invariant the density of $\kappa$ is also rotational invariant, hence the fundamental solution is rotational invariant and can be described with the help of $d_{\psi}(\xi-\eta)=g\left(\|\xi-\eta\|^{2}\right)$ for a suitable function $g:[0, \infty) \rightarrow \mathbb{R} \cup\{+\infty\}$ which in turn allows to study $E * h$ more closely. While in general we have no result stating that the density of $\kappa$ is a function of $d_{\psi}$, we can provide new and interesting non-trivial examples where this result holds. For example for $\psi(\xi, \eta)=|\xi|+|\eta|, \xi, \eta \in \mathbb{R}$, and symbols $f(\psi(\xi, \eta))=f(|\xi|+|\eta|)$, where $f$ belongs to a suitable class of Bernstein functions the result can be proved.

Hence, combining the theory of L.Schwartz distributions with potential theory, with the theory of $\psi$-Bessel potential spaces and with properties of the metric measure space $\left(\mathbb{R}^{n}, d_{\psi}, \lambda^{(n)}\right)$ leads to some (first) new insights on regularity properties of solutions to the equation $\psi(D) u=f$ for $\psi$ satisfying the assumptions i) $-i v)$.

Here is a brief summary of the main chapters: In Chapter 1 we recollect main results from Fourier analysis including the Fourier analytical treatment of convolution semigroups, e.g. negative definite functions and the Lévy-Khinchin formula where the proof can be found in [25], along with Bochner's theory of subordination where the original proof is found in [5]. Chapter 2 deals with the essential background knowledge from functional analysis, in particular oneparameter semigroups of operators. It also includes a section on Dirichlet spaces as well as one on pseudo-differential operators with negative definite symbols. The latter class of operators allows us to construct certain Dirichlet forms given certain additional assumptions. The example provided in Theorem 2.4.12 is new and seems to be helpful to extend results obtained later in this thesis for translation invariant operators to those with variable coefficients. The most useful books on semigroup theory are given by [26], [32] and [38] where we consider 
mainly [7]. In Chapter 3 we introduce and discuss metric measure spaces related to continuous negative definite functions. Here we rely mainly on [23] where an introduction to the analysis on metric measure spaces can also be found in the book by Heinonen [13].

In Chapter 4 we first summarise important results from the theory of distributions and their applications to fundamental solutions of linear partial differential operators with constant coefficients. The aim is to transfer some of these results to pseudo-differential operators with negative definite symbols. These operators we will deal within an $L^{2}$-theory and the corresponding function spaces are introduced in Paragraph 4.3. With these preparations we eventually can prove one of our main results as described above, i.e. Theorem 4.4.4 and Corollary 4.4.10. In Chapter 5 we make use that we are working with operators generating extended Dirichlet spaces. Background material is presented in the first two paragraphs of this chapter, in Paragraph 5.3 we then identify fundamental solutions with potential kernels, and derive related properties, see Theorem 5.3.1 and Theorem 5.3.2. In the last paragraph of this chapter we discuss some comparison results for transition densities, we in particular are interested in getting first ideas how freezing the coefficient techniques might work in our context, and for this we will use our example from Theorem 2.4.12. Our final chapter serves three purposes. Firstly, we use the results we obtained with the help of the theory of distributions where we combine this with those obtained with the help of the theory of (extended) Dirichlet spaces and the associated metric measure spaces to give a further insight with regard to the equation $\psi(D) u=f$. The main part of Chapter 6 consists of providing examples, where some of these examples allow us to handle rather anisotropic continuous negative definite functions, therefore are leading far away from existing results. In the examples we give details for the calculations leading to $\frac{1}{\psi} \in L_{l o c}^{1}\left(\mathbb{R}^{n}\right)$ and make a short remark to the changes needed to obtain $\frac{1}{\psi} \in L_{l o c}^{2}\left(\mathbb{R}^{n}\right)$. This is justified by the fact that we hereby also provide concrete (new) examples for extended Dirichlet spaces. Of particular interest are Example 6.2.10, and Example 6.2.11. In the final paragraph we outline some ideas for further investigations. 


\section{Chapter 1}

\section{Fourier Analysis}

\subsection{Fourier Transform}

We will begin by discussing the Fourier transform in $\mathcal{S}\left(\mathbb{R}^{n}\right)$. We need the following definition which is taken from [20].

Definition 1.1.1. A. The Schwartz space $\mathcal{S}\left(\mathbb{R}^{n}\right)$ of functions $\varphi: \mathbb{R}^{n} \rightarrow \mathbb{C}$ is defined as

$$
\mathcal{S}\left(\mathbb{R}^{n}\right)=\left\{\varphi \in \mathrm{C}^{\infty}\left(\mathbb{R}^{n}\right): p_{\alpha, \beta}(\varphi)<\infty, \forall \alpha, \beta \in \mathbb{N}_{0}^{n}\right\}
$$

where

$$
p_{\alpha, \beta}(\varphi):=\|\varphi\|_{\alpha, \beta}=\sup _{x \in \mathbb{R}^{n}}\left|x^{\beta} \partial^{\alpha} \varphi(x)\right| .
$$

B. A sequence of functions $\left(\varphi_{k}\right)_{k \in \mathbb{N}}, \varphi_{k} \in \mathcal{S}\left(\mathbb{R}^{n}\right)$, converges to a function $\varphi$ in $\mathcal{S}\left(\mathbb{R}^{n}\right)$ if for all multi-indices $\alpha, \beta$ we have

$$
\left\|\varphi_{k}-\varphi\right\|_{\alpha, \beta} \rightarrow 0 \text { as } k \rightarrow \infty
$$

We say that Schwartz space functions are rapidly decreasing. When there is no ambiguity we will write $\mathcal{S}\left(\mathbb{R}^{n}\right)$ as $\mathcal{S}$.

Example 1.1.2. The function $f(x)=e^{-|x|^{2}}$ belongs to $\mathcal{S}\left(\mathbb{R}^{n}\right)$. More generally, if $p$ is any polynomial, then $g(x)=p(x) e^{-|x|^{2}}$ belongs to $\mathcal{S}$. 
Example 1.1.3. The function

$$
f(x)=\frac{1}{\left(1+|x|^{2}\right)^{k}}
$$

does not belong to $\mathcal{S}$ for any $k \in \mathbb{N}$ since $|x|^{2 k} f(x)$ does not decay to zero as $|x| \rightarrow \infty$.

Now, we will look at the Fourier transform in $\mathcal{S}\left(\mathbb{R}^{n}\right)$.

Definition 1.1.4. Let $u \in \mathcal{S}\left(\mathbb{R}^{n}\right)$. The Fourier transform of $u$ is defined by

$$
\hat{u}(\xi):=(2 \pi)^{\frac{-n}{2}} \int_{\mathbb{R}^{n}} e^{-i x \cdot \xi} u(x) d x .
$$

We will also write $F(u)(\xi)$ for $\hat{u}(\xi)$ and state some properties of the Fourier transform taken from [20].

Lemma 1.1.5. A) Let $T_{a}: \mathbb{R}^{n} \rightarrow \mathbb{R}^{n}$ be the translation operator $T_{a} x=a+x$, $a \in \mathbb{R}^{n}$. For $u \in \mathcal{S}\left(\mathbb{R}^{n}\right)$ it follows that

$$
\left(\widehat{u \circ T_{a}}\right)(\xi)=e^{i a \cdot \xi} \hat{u}(\xi) .
$$

B) Let $T: \mathbb{R}^{n} \rightarrow \mathbb{R}^{n}$ be a bijective linear mapping, then for $u \in \mathcal{S}\left(\mathbb{R}^{n}\right)$ the function $u \circ T$ belongs to $\mathcal{S}\left(\mathbb{R}^{n}\right)$ too, and for its Fourier transform we find

$$
(\widehat{u \circ T})(\xi)=\frac{1}{|\operatorname{det} T|} \hat{u} \circ\left(T^{-1}\right)^{t}(\xi) \text {. }
$$

C) For $u \in \mathcal{S}\left(\mathbb{R}^{n}\right)$ we have

$$
\overline{\hat{u}(\xi)}=F^{-1}(\bar{u})(\xi)
$$

D) For $u, v \in \mathcal{S}\left(\mathbb{R}^{n}\right)$ we have the convolution theorem

$$
(\widehat{u * v})(\xi)=(2 \pi)^{\frac{n}{2}} \hat{u}(\xi) \cdot \hat{v}(\xi)
$$

Next, we will look at the Fourier transform in $L^{p}\left(\mathbb{R}^{n}\right)$. We begin by looking at the case where $p=1$. For $u \in L^{1}\left(\mathbb{R}^{n}\right)$ the function $x \mapsto e^{-i x \cdot \xi} u(x)$ is an element of $L^{1}\left(\mathbb{R}^{n}\right)$, therefore its integral is well defined and we set

$$
\hat{u}(\xi)=(2 \pi)^{\frac{-n}{2}} \int_{\mathbb{R}^{n}} e^{-i x \cdot \xi} u(x) d x .
$$


We call $\hat{u}$ the Fourier transform of $u \in L^{1}\left(\mathbb{R}^{n}\right)$, where we also note that $\mathcal{S}\left(\mathbb{R}^{n}\right) \subset$ $L^{1}\left(\mathbb{R}^{n}\right)$.

A key result regarding the Fourier transform in $L^{2}$ is that of Plancherel's theorem.

Theorem 1.1.6. If $u \in L^{2}\left(\mathbb{R}^{n}\right) \cap L^{1}\left(\mathbb{R}^{n}\right)$, then $\hat{u} \in L^{2}\left(\mathbb{R}^{n}\right)$ and

$$
\|\hat{u}\|^{2}=\|u\|^{2}
$$

We will now state a sesquilinear version of the Parseval's identity.

Corollary 1.1.7. If $u \in L^{2}\left(\mathbb{R}^{n}\right)$ and $v \in L^{2}\left(\mathbb{R}^{n}\right)$, then

$$
(\hat{u}, \hat{v})=(u, v)
$$

We will find the next result useful which is taken from [10].

Theorem 1.1.8. $C_{0}^{\infty}\left(\mathbb{R}^{n}\right)$ is dense in $L^{2}\left(\mathbb{R}^{n}\right)$.

Proof. Let $f \in L^{2}\left(\mathbb{R}^{n}\right)$ be given, and define $f_{\epsilon}$ by

$$
f_{\epsilon}(x)=f * j_{\epsilon}=\int f(y) j_{\epsilon}(x-y) d y,
$$

where

$$
j_{\epsilon}(x)=\epsilon^{-n} j\left(\frac{x}{\epsilon}\right), \epsilon>0 .
$$

Choose some $\phi \in C_{0}^{\infty}\left(\mathbb{R}^{n}\right)$ such that $0 \leq \phi \leq 1$ and $\phi=1$ for $|x| \leq 1$, and put $g_{\epsilon}(x)=\phi(\epsilon x) f_{\epsilon}(x)$. Then $g_{\epsilon} \in C_{0}^{\infty}\left(\mathbb{R}^{n}\right)$, and

$$
\begin{aligned}
\left\|f-g_{\epsilon}\right\| & \leq\left\|\left(f-f_{\epsilon}\right) \phi(\epsilon x)\right\|+\|(\phi(\epsilon x)-1) f\| \\
& \leq\left\|f-f_{\epsilon}\right\|+\left(\int_{|x|>\epsilon^{-1}}|f(x)|^{2} d x\right)^{\frac{1}{2}} .
\end{aligned}
$$

In the last expression, the first term tends to 0 , and the second one does so because $f \in L^{2}\left(\mathbb{R}^{n}\right)$.

For a function $u \in L^{2}\left(\mathbb{R}^{n}\right)$ the integral

$$
(2 \pi)^{\frac{-n}{2}} \int_{\mathbb{R}^{n}} e^{-i x \cdot \xi} u(x) d x
$$


does not in general converge. Therefore the Fourier transform cannot be defined for $u \in L^{2}\left(\mathbb{R}^{n}\right)$ by the above. To obtain an extension of the Fourier transform from $L^{1}\left(\mathbb{R}^{n}\right) \cap L^{2}\left(\mathbb{R}^{n}\right)$ onto $L^{2}\left(\mathbb{R}^{n}\right)$, we will make use of Plancherel's theorem, Theorem 1.1.6.

Theorem 1.1.9. The Fourier transform as it is defined on $L^{1}\left(\mathbb{R}^{n}\right) \cap L^{2}\left(\mathbb{R}^{n}\right)$ has an extension to $L^{2}\left(\mathbb{R}^{n}\right)$. This extension is an isometry on $L^{2}\left(\mathbb{R}^{n}\right)$ which is bijective and has a continuous inverse. We will denote this extension again by $\hat{u}$ or $F u$.

We want to extend the Fourier transform to $L^{p}\left(\mathbb{R}^{n}\right)$ and for this we need the Riesz-Thorin convexity theorem, see [6] or [39].

Theorem 1.1.10. A) Let $X$ be a Hausdorff topological space containing all spaces $L^{p}\left(\mathbb{R}^{n}\right), 1 \leq p \leq \infty$, in the sense of continuous embeddings. Let $T$ be a linear operator acting from $X$ into $X$ and for $1 \leq p_{1}, p_{2}, q_{1}, q_{2} \leq \infty$ suppose that $\left.T\right|_{L^{p_{j}}\left(\mathbb{R}^{n}\right)}: L^{p_{j}}\left(\mathbb{R}^{n}\right) \rightarrow L^{q_{j}}\left(\mathbb{R}^{n}\right)$ is continuous, $\|T\|_{L^{p_{j}, L^{q_{j}}}}=$ : $M_{j}, j=1,2$. For $\theta \in[0,1]$ define $p_{\theta}$ and $q_{\theta}$ by

$$
\frac{1}{p_{\theta}}=\frac{1-\theta}{p_{1}}+\frac{\theta}{p_{2}} \text { and } \frac{1}{q_{\theta}}=\frac{1-\theta}{q_{1}}+\frac{\theta}{q_{2}} .
$$

Then $\left.T\right|_{L^{p_{\theta}\left(\mathbb{R}^{n}\right)}}: L^{p_{\theta}}\left(\mathbb{R}^{n}\right) \rightarrow L^{q_{\theta}}\left(\mathbb{R}^{n}\right)$ is continuous with operator norm $M_{\theta}$ satisfying

$$
M_{\theta} \leq M_{1}^{1-\theta} M_{2}^{\theta}
$$

B) Let $1 \leq p_{1}, p_{2}, q_{1}, q_{2} \leq \infty$ and let $T$ be a linear operator from $L^{p_{1}}\left(\mathbb{R}^{n}\right) \cap$ $L^{p_{2}}\left(\mathbb{R}^{n}\right)$ to $L^{q_{1}}\left(\mathbb{R}^{n}\right)+L^{q_{2}}\left(\mathbb{R}^{n}\right)$ satisfying $\|T\|_{L^{p_{j}, L^{q_{j}}}}=M_{j}<\infty, j=1,2$. Then for $p_{\theta}$ and $q_{\theta}$, as defined above, the operator $\left.T\right|_{L^{p_{1}} \cap L^{p_{2}}}$ satisfies the estimate

$$
\|T u\|_{q_{\theta}} \leq M_{1}^{1-\theta} M_{2}^{\theta}\|u\|_{p_{\theta}}
$$

and therefore extends to a continuous operator from $L^{p_{\theta}}\left(\mathbb{R}^{n}\right)$ to $L^{q_{\theta}}\left(\mathbb{R}^{n}\right)$.

For $\theta \in[0,1]$ we set

$$
p_{\theta}=\frac{2}{1+\theta} \text { and } p_{\theta}^{\prime}=\frac{2}{1-\theta},
$$


hence, $1 \leq p_{\theta} \leq 2$ and $\frac{1}{p_{\theta}}+\frac{1}{p_{\theta}^{\prime}}=1$. Therefore using the Riesz-Thorin convexity theorem, Theorem 1.1.10, gives the Hausdorff-Young theorem which is given by

Theorem 1.1.11. The Fourier transform extends to a continuous linear mapping from $L^{p_{\theta}}\left(\mathbb{R}^{n}\right)$ into $L^{p_{\theta}^{\prime}}\left(\mathbb{R}^{n}\right)$ with norm less or equal to $(2 \pi)^{-n \frac{\theta}{2}}$, where $p_{\theta}$ is given by (1.1).

The inverse Fourier transform of $\hat{u}$ is given by:

Theorem 1.1.12. For $u \in \mathcal{S}\left(\mathbb{R}^{n}\right)$ we have for all $x \in \mathbb{R}^{n}$

$$
\left(F^{-1} u\right)(x)=(2 \pi)^{\frac{-n}{2}} \int_{\mathbb{R}^{n}} e^{i x \cdot \xi} \hat{u}(\xi) d \xi
$$

In general we call for $v \in \mathcal{S}\left(\mathbb{R}^{n}\right)$

$$
\left(F^{-1} v\right)(x):=(2 \pi)^{-\frac{n}{2}} \int_{\mathbb{R}^{n}} e^{i x \cdot \xi} v(\xi) d \xi
$$

the inverse Fourier transform of $v$.

A continuous functional on $\mathcal{S}$ is a linear map $f: \mathcal{S}\left(\mathbb{R}^{n}\right) \rightarrow \mathbb{C}, \varphi \rightarrow\langle f, \varphi\rangle$ such that for any sequence $\left(\varphi_{k}\right)_{k \in \mathbb{N}}, \varphi_{k} \in \mathcal{S}\left(\mathbb{R}^{n}\right)$, the property $p_{\alpha, \beta}\left(\varphi_{k}\right) \rightarrow 0$ for all $\alpha, \beta \in \mathbb{N}_{0}^{n}$, implies $\left\langle f, \varphi_{k}\right\rangle \rightarrow 0$. Given two topological vector spaces $X$ and $Y$, we say that $(A, D(A))$ is a linear operator from $X$ to $Y$ if $D(A) \subset X$ is a linear subspace and $A: D(A) \rightarrow Y$ is linear. Linear operators $(l, D(l))$ from a vector space $X$ to $\mathbb{C}($ or $\mathbb{R})$ are called linear functionals.

Definition 1.1.13. The space of continuous linear functionals on $\mathcal{S}\left(\mathbb{R}^{n}\right)$ is $\mathcal{S}^{\prime}\left(\mathbb{R}^{n}\right)$, the space of tempered distributions.

On $\mathcal{S}^{\prime}\left(\mathbb{R}^{n}\right)$ we will consider the weak-*-topology. Note that $L^{p}\left(\mathbb{R}^{n}\right) \subset \mathcal{S}^{\prime}\left(\mathbb{R}^{n}\right)$, as well as $C_{b}\left(\mathbb{R}^{n}\right) \subset \mathcal{S}^{\prime}\left(\mathbb{R}^{n}\right)$ and the bounded Borel measures belong to $\mathcal{S}^{\prime}\left(\mathbb{R}^{n}\right)$. We extend the Fourier transform from $\mathcal{S}\left(\mathbb{R}^{n}\right)$ to $\mathcal{S}^{\prime}\left(\mathbb{R}^{n}\right)$ by

Definition 1.1.14. For $u \in \mathcal{S}^{\prime}\left(\mathbb{R}^{n}\right)$, the Fourier transform $\hat{u}$ of $u$ is defined by

$$
\langle\hat{u}, \phi\rangle=\langle u, \hat{\phi}\rangle \text { for all } \phi \in \mathcal{S}\left(\mathbb{R}^{n}\right) \text {. }
$$


The Fourier transform is an automorphism of the space of tempered distributions, as well as of the Schwartz space.

Since the bounded Borel measures $\mu \in \mathcal{M}_{b}^{+}\left(\mathbb{R}^{n}\right)$ belong to $\mathcal{S}^{\prime}\left(\mathbb{R}^{n}\right)$, i.e. $\mathcal{M}_{b}^{+}\left(\mathbb{R}^{n}\right) \subset$ $\mathcal{S}^{\prime}\left(\mathbb{R}^{n}\right)$, the Fourier transform $\hat{\mu}$ of $\mu$ is well defined and for $\phi \in \mathcal{S}\left(\mathbb{R}^{n}\right)$ we have

$$
\langle\hat{\mu}, \phi\rangle=\langle\mu, \hat{\phi}\rangle=\int_{\mathbb{R}^{n}} \hat{\phi}(\xi) \mu(d \xi) .
$$

From (1.2) and by the definition of $\hat{\phi}$ we get, using a version of Fubini's theorem,

$$
\begin{aligned}
\langle\hat{\mu}, \phi\rangle & =(2 \pi)^{\frac{-n}{2}} \int_{\mathbb{R}^{n}} \int_{\mathbb{R}^{n}} e^{-i x \cdot \xi} \phi(x) d x \mu(d \xi) \\
& =(2 \pi)^{\frac{-n}{2}} \int_{\mathbb{R}^{n}}\left(\int_{\mathbb{R}^{n}} e^{-i x \cdot \xi} \mu(d \xi)\right) \phi(x) d x \\
& =\left\langle(2 \pi)^{\frac{-n}{2}} \int_{\mathbb{R}^{n}} e^{-i(., \xi)} \mu(d \xi), \phi\right\rangle .
\end{aligned}
$$

Hence we have

$$
\hat{\mu}(\xi)=(2 \pi)^{\frac{-n}{2}} \int_{\mathbb{R}^{n}} e^{-i x \cdot \xi} \mu(d x)
$$

which is the Fourier transform of bounded Borel measures $\mu \in \mathcal{M}_{b}^{+}\left(\mathbb{R}^{n}\right)$.

The following theorem states some properties of the Fourier transform on $\mathcal{M}_{b}^{+}\left(\mathbb{R}^{n}\right)$.

Theorem 1.1.15. A) For a linear mapping $T: \mathbb{R}^{n} \rightarrow \mathbb{R}^{n}$ we have for the image $T(\mu)$ of the measure $\mu \in \mathcal{M}_{b}^{+}\left(\mathbb{R}^{n}\right)$

$$
\widehat{[T(\mu)}]=\hat{\mu} \circ T^{t}
$$

B) For the translation $T_{a}: \mathbb{R}^{n} \rightarrow \mathbb{R}^{n}, x \mapsto x+a$, it follows that

$$
\left.\widehat{\left[T_{a}(\mu)\right.}\right]=e^{-i \xi \cdot a} \hat{\mu}
$$

C) For $\mu, \nu \in \mathcal{M}_{b}^{+}$the convolution theorem holds, i.e.

$$
\widehat{(\mu * \nu)}=(2 \pi)^{\frac{n}{2}} \hat{\mu} \cdot \hat{\nu}
$$

D) For $\mu \in \mathcal{M}_{b}^{+}\left(\mathbb{R}^{n}\right)$ and $\nu \in \mathcal{M}_{b}^{+}\left(\mathbb{R}^{m}\right)$ we have

$$
\widehat{(\mu \otimes \nu})(\xi, \eta)=\hat{\mu}(\xi) \cdot \hat{\nu}(\eta), \xi \in \mathbb{R}^{n} \text { and } \eta \in \mathbb{R}^{m}
$$


The positive definite functions are needed to characterise the Fourier transform of bounded measures.

Definition 1.1.16. A function $u: \mathbb{R}^{n} \rightarrow \mathbb{C}$ is called positive definite if for any choice of $k \in \mathbb{N}$ and vectors $\xi^{1}, \ldots ., \xi^{k} \in \mathbb{R}^{n}$ the matrix $\left(u\left(\xi^{j}-\xi^{l}\right)\right)_{j, l=1, \ldots, k}$ is positive Hermitian, i.e. for all $\lambda_{1}, \ldots ., \lambda_{k} \in \mathbb{C}$ we have

$$
\sum_{j, l=1}^{k} u\left(\xi^{j}-\xi^{l}\right) \lambda_{j} \bar{\lambda}_{l} \geq 0 .
$$

Lemma 1.1.17. Let $\mu \in \mathcal{M}_{b}^{+}\left(\mathbb{R}^{n}\right)$. Then $\hat{\mu}$ is a positive definite function.

Proof. For $k \in \mathbb{N}$ and $\xi^{1}, \ldots, \xi^{k} \in \mathbb{R}^{n}$ we find with $\lambda_{1}, \ldots, \lambda_{k} \in \mathbb{C}$

$$
\begin{aligned}
& \sum_{j, l=1}^{k} \lambda_{j} \overline{\lambda_{l}} \hat{\mu}\left(\xi^{j}-\xi^{l}\right)=(2 \pi)^{\frac{-n}{2}} \int_{\mathbb{R}^{n}} \sum_{j, l=1}^{k} \lambda_{j} \overline{\lambda_{l}} e^{-i\left(\xi^{j}-\xi^{l}\right) \cdot x} \mu(d x) \\
& =(2 \pi)^{\frac{-n}{2}} \int_{\mathbb{R}^{n}}\left(\sum_{j=1}^{k} \lambda_{j} e^{-i \xi^{j} \cdot x}\right) \cdot \overline{\left(\sum_{l=1}^{k} \lambda_{l} e^{-i \xi^{l} \cdot x}\right)} \mu(d x) \\
& =(2 \pi)^{\frac{-n}{2}} \int_{\mathbb{R}^{n}}\left|\sum_{j=1}^{k} \lambda_{j} e^{-i \xi^{j} \cdot x}\right|^{2} \mu(d x) \geq 0
\end{aligned}
$$

One of the most important results on positive definite functions and their characterisation in terms of Fourier transforms which was established by Bochner in 1932 , says that every continuous, positive definite function $u: \mathbb{R}^{n} \rightarrow \mathbb{C}$ is the Fourier transform of a measure $\mu \in \mathcal{M}_{b}^{+}\left(\mathbb{R}^{n}\right)$. This is stated formally in the following theorem; see Theorem 3.5.7 in [20]:

Theorem 1.1.18. A function $u: \mathbb{R}^{n} \rightarrow \mathbb{C}$ is the Fourier transform of a measure $\mu \in \mathcal{M}_{b}^{+}\left(\mathbb{R}^{n}\right)$ with total mass $\|\mu\|$, if and only if the following conditions are fulfilled

1) $u$ is continuous.

2) $u(0)=\hat{u}(0)=(2 \pi)^{\frac{-n}{2}}\|u\|$.

3) $u$ is positive definite. 


\subsection{Negative Definite Functions and Convolu- tion Semigroups}

We start with

Definition 1.2.1. The sequence $\left(\mu_{\nu}\right)_{\nu \in \mathbb{N}}$ of bounded measures $\mu_{k} \in \mathcal{M}_{b}$ converges weakly to $\mu_{0} \in \mathcal{M}_{b}$ if for all $u \in C_{b}(G ; \mathbb{R})$ we have

$$
\lim _{\nu \rightarrow \infty} \int_{G} u(x) \mu_{\nu}(d x)=\int_{G} u(x) \mu_{0}(d x) .
$$

Whenever (1.3) holds only for all $u \in C_{\infty}(G ; \mathbb{R})$, the sequence $\left(\mu_{\nu}\right)_{\nu \in \mathbb{N}}$ is said to converge with respect to $C_{\infty}$ to $\mu_{0}$, but if (1.3) holds only for all $u \in C_{0}(G ; \mathbb{R})$, we say that $\left(\mu_{\nu}\right)_{\nu \in \mathbb{N}}$ converges vaguely to $\mu_{0}$.

For $\mu_{1}, \mu_{2} \in \mathcal{M}_{b}^{+}\left(\mathbb{R}^{n}\right)$ and any non-negative measurable function on $\mathbb{R}^{n}$ we have

$$
\begin{aligned}
\int_{\mathbb{R}^{n}} f(x)\left(\mu_{1} * \mu_{2}\right)(d x) & =\int_{\mathbb{R}^{n}} \int_{\mathbb{R}^{n}} f(x+y) \mu_{1}(d x) \mu_{2}(d y) \\
& =\int_{\mathbb{R}^{n}} \int_{\mathbb{R}^{n}} f(x+y) \mu_{2}(d y) \mu_{1}(d x)
\end{aligned}
$$

which yields

$$
\left(\mu_{1} * \mu_{2}\right)(B)=\int_{\mathbb{R}^{n}} \mu_{1}(B-y) \mu_{2}(d y)=\int_{\mathbb{R}^{n}} \mu_{2}(B-z) \mu_{1}(d z)
$$

for all $B \in \mathcal{B}^{(n)}$.

Definition 1.2.2. A family $\left(\mu_{t}\right)_{t \geq 0}$ of bounded Borel measures on $\mathbb{R}^{n}$ is called a convolution semigroup on $\mathbb{R}^{n}$ if the following conditions are fulfilled

$$
\begin{aligned}
& \mu_{t}\left(\mathbb{R}^{n}\right) \leq 1 \text { for all } t \geq 0 \\
& \mu_{s} * \mu_{t}=\mu_{t+s} \text { s, } t \geq 0 \text { and } \mu_{0}=\varepsilon_{0} \\
& \mu_{t} \rightarrow \varepsilon_{0} \text { vaguely as } t \rightarrow 0
\end{aligned}
$$

where $\varepsilon_{0}$ is the Dirac measure at zero.

Next, we will look at the definition of a negative definite function, where the results are taken from [20]. The concept and definition of negative definite functions is due to I.J.Schoenberg [36], who introduced it in connection with isometric embeddings of metric spaces into Hilbert spaces. 
Definition 1.2.3. A function $\psi: \mathbb{R}^{n} \rightarrow \mathbb{C}$ is called negative definite if

$$
\psi(0) \geq 0
$$

and

$$
\xi \mapsto(2 \pi)^{\frac{-n}{2}} e^{-t \psi(\xi)} \text { is positive definite for } t \geq 0 \text {. }
$$

For any convolution semigroup $\left(\mu_{t}\right)_{t \geq 0}$, its Fourier transform can be characterised by a continuous negative definite function, where the following theorem holds.

Theorem 1.2.4. Let $\left(\mu_{t}\right)_{t \geq 0}$ be a convolution semigroup on $\mathbb{R}^{n}$. Then there exists an unique continuous negative definite function $\psi: \mathbb{R}^{n} \rightarrow \mathbb{C}$ such that

$$
\hat{\mu}_{t}(\xi)=(2 \pi)^{\frac{-n}{2}} e^{-t \psi(\xi)}
$$

holds for all $\xi \in \mathbb{R}^{n}$ and $t \geq 0$.

Note that $\psi$ is real-valued if and only if all measures $\mu_{t}$ are symmetric.

We will now introduce the classes $N\left(\mathbb{R}^{n}\right)$ and $C N\left(\mathbb{R}^{n}\right)$, so that we can study negative definite functions in more detail.

Definition 1.2.5. A function $\psi: \mathbb{R}^{n} \rightarrow \mathbb{C}$ belongs to the class $N\left(\mathbb{R}^{n}\right)$ if for any choice of $k \in \mathbb{N}$ and vectors $\xi^{1}, \ldots, \xi^{k} \in \mathbb{R}^{n}$ the matrix

$$
\left(\psi\left(\xi^{j}\right)+\overline{\psi\left(\xi^{l}\right)}-\psi\left(\xi^{j}-\xi^{l}\right)\right)_{j, l=1, \ldots, k}
$$

is positive Hermitian. Further we set

$$
C N\left(\mathbb{R}^{n}\right):=N\left(\mathbb{R}^{n}\right) \cap C\left(\mathbb{R}^{n}\right) .
$$

Theorem 1.2.6. [20] A function $\varphi: \mathbb{R}^{n} \rightarrow \mathbb{C}$ is an element of $N\left(\mathbb{R}^{n}\right)$ if and only if it is negative definite.

Corollary 1.2.7. Let $\psi: \mathbb{R}^{n} \rightarrow \mathbb{C}$ be a negative definite function. Then $\frac{1}{\psi+\varepsilon}$ is for all $\varepsilon>0$ a positive definite function. 
We will now give some elementary properties of negative definite functions. The set of all continuous negative definite functions is a convex cone which is closed under locally uniform convergence. Moreover, if $\psi$ is real-valued it is non-negative and further, for $\psi \in C N\left(\mathbb{R}^{n}\right)$ exists a constant $c_{\psi}$ such that for all $\xi \in \mathbb{R}^{n}$ we have $\psi(\xi) \leq c_{\psi}\left(1+|\xi|^{2}\right)$.

Lemma 1.2.8. For any $\psi \in N\left(\mathbb{R}^{n}\right)$ we have

$$
\begin{aligned}
& \sqrt{|\psi(\xi+\eta)|} \leq \sqrt{|\psi(\xi)|}+\sqrt{|\psi(\eta)|} \\
& \mid \sqrt{|\psi(\xi)|}-\sqrt{|\psi(\eta)|} \leq \sqrt{|\psi(\xi-\eta)|} \\
& \text { and } \\
& |\psi(\xi)+\psi(\eta)-\psi(\xi+\eta)| \leq 2(\operatorname{Re} \psi(\xi))^{\frac{1}{2}}(\operatorname{Re} \psi(\eta))^{\frac{1}{2}}
\end{aligned}
$$

Proof. We will give a proof of the first inequality. Firstly, for any $\xi, \eta \in \mathbb{R}^{n}$ we will take the matrix

$$
\left(\begin{array}{cc}
\psi(\xi)+\overline{\psi(\xi)}-\psi(0) & \psi(\xi)+\overline{\psi(\eta)}-\psi(\xi-\eta) \\
\psi(\eta)+\overline{\psi(\xi)}-\psi(\eta-\xi) & \psi(\eta)+\overline{\psi(\eta)}-\psi(0)
\end{array}\right)
$$

where the determinant is non-negative. Since $\psi=\bar{\psi}$ and $\psi(0) \geq 0$ it follows that

$$
|\psi(\xi)+\overline{\psi(\eta)}-\psi(\xi-\eta)|^{2} \leq 4 \operatorname{Re} \psi(\xi) \operatorname{Re} \psi(\eta) \leq 4|\psi(\xi)||\psi(\eta)|
$$

We may also take $-\eta$, and observing that $|\psi(\eta)|=|\psi(-\eta)|$ we get

$$
|\psi(\xi)+\psi( \pm \eta)-\psi(\xi \pm \eta)|^{2} \leq 4|\psi(\xi)||\psi(\eta)|
$$

and further we have

$$
\begin{aligned}
& |\psi(\xi \pm \eta)|-|\psi(\xi)|-|\psi( \pm \eta)| \leq|\psi(\xi \pm \eta)|-|\psi(\xi)+\psi( \pm \eta)| \\
& \leq|\psi(\xi)+\psi( \pm \eta)-\psi(\xi \pm \eta)| \leq 2|\psi(\xi)|^{\frac{1}{2}}|\psi(\eta)|^{\frac{1}{2}}
\end{aligned}
$$

which yields $|\psi(\xi+\eta)| \leq\left(|\psi(\xi)|^{\frac{1}{2}}+|\psi(\eta)|^{\frac{1}{2}}\right)^{2}$ implying (1.4).

We will now show that $d_{\psi}(\xi, \eta)=\sqrt{|\psi(\xi-\eta)|}$ is a metric provided $\psi(\xi)=0$ if and only if $\xi=0$. To do this we need to give the definition of a metric. 
Definition 1.2.9. Let $X \neq \emptyset$ be a set. We call a mapping $d: X \times X \rightarrow \mathbb{R} a$ metric on $X$ and $(X, d)$ a metric space if

i) $d(x, y) \geq 0$ for all $x, y \in X$ and $d(x, y)=0$ if and only if $x=y$;

ii) $d(x, y)=d(y, x)$ for all $x, y \in X$;

iii) $d(x, y) \leq d(x, z)+d(z, y)$ for all $x, y, z \in X$.

Lemma 1.2.10. Let $\psi: \mathbb{R}^{n} \rightarrow \mathbb{C}$ be a negative definite function. Then $d_{\psi}(\xi, \eta)=$ $\sqrt{|\psi(\xi-\eta)|}$ is a metric if and only if $\psi(\xi)=0$ implies $\xi=0$.

Proof. Firstly, we have $d_{\psi}(\xi, \eta) \geq 0$ and $d_{\psi}(\xi, \eta)=0$ if and only if $\sqrt{|\psi(\xi-\eta)|}=$ 0 , i.e. $\xi=\eta$. This requires the condition where $\psi(\xi)=0$ if and only if $\xi=0$. Moreover, we find

$$
d_{\psi}(\xi, \eta)=\sqrt{|\psi(\xi-\eta)|}=\sqrt{|\psi(-(\xi-\eta))|}=\sqrt{|\psi(\eta-\xi)|}=d_{\psi}(\eta, \xi)
$$

We find further that

$$
\begin{aligned}
d_{\psi}(\xi, \gamma) & =\sqrt{|\psi(\xi-\gamma)|}=\sqrt{|\psi(\xi-\eta+\eta-\gamma)|} \\
& \leq \sqrt{|\psi(\xi-\eta)|}+\sqrt{|\psi(\eta-\gamma)|}=d_{\psi}(\xi, \eta)+d_{\psi}(\eta, \gamma)
\end{aligned}
$$

Hence, the triangle inequality is satisfied proving that $d_{\psi}(\xi, \eta)=\sqrt{|\psi(\xi-\eta)|}$ is a metric on $\mathbb{R}^{n}$.

The following lemma, often called Peetre's inequality which is taken from [9], indicates the closeness of continuous negative definite functions to metrics.

Lemma 1.2.11. Let $\psi: \mathbb{R}^{n} \rightarrow \mathbb{C}$ be a negative definite function. Then we have

$$
\frac{1+|\psi(\xi)|}{1+|\psi(\eta)|} \leq 2(1+|\psi(\xi-\eta)|)
$$

We also have,

$$
1+|\psi(\xi \pm \eta)| \leq(1+|\psi(\xi)|)(1+\sqrt{|\psi(\eta)|})^{2}
$$




\subsection{Lévy-Khinchin Formula}

The Lévy-Khinchin formula was presented by Paul Lévy in (1934) and later a much simpler proof was given by Aleksandr Khinchin (1937). Every continuous negative definite function admits a Lévy Khinchin representation, as is stated in the theorem below; see Theorem 1.1.5 in [20].

Theorem 1.3.1. The Lévy-Khinchin formula states that every continuous negative definite function $\psi: \mathbb{R}^{n} \rightarrow \mathbb{C}$ has the representation

$$
\begin{aligned}
\psi(\xi) & =c+i(d \cdot \xi)+q(\xi)+ \\
& +\int_{\mathbb{R}^{n} \backslash\{0\}}\left(1-e^{-i x \xi}-\frac{i x \cdot \xi}{1+|x|^{2}}\right) \frac{1+|x|}{|x|^{2}} \mu(d x)
\end{aligned}
$$

with a non-negative constant $c \geq 0$, a vector $d \in \mathbb{R}^{n}$, a symmetric positive semidefinite quadratic form $q$, and a finite Borel measure $\mu$ on $\mathbb{R}^{n} \backslash\{0\}$.

Definition 1.3.2. Let $\mu$ be the measure in the Lévy-Khinchin representation of the continuous negative definite function $\psi: \mathbb{R}^{n} \rightarrow \mathbb{C}$. The measure

$$
\nu(d x)=\frac{1+|x|^{2}}{|x|^{2}} \mu(d x)
$$

defined on $\mathcal{B}\left(\mathbb{R}^{n} \backslash\{0\}\right)$ is called the Lévy measure associated with $\psi$.

The measure $\nu$ is a Radon measure on $\mathbb{R}^{n} \backslash\{0\}$ satisfying the integrability condition

$$
\int_{\mathbb{R}^{n} \backslash\{0\}}\left(|x|^{2} \wedge 1\right) \nu(d x)<\infty
$$

We can use the Lévy measure when working with the Lévy-Khinchin formula and write

$$
\psi(\xi)=c+i(d \cdot \xi)+q(\xi)+\int_{\mathbb{R}^{n} \backslash\{0\}}\left(1-e^{i x \xi}-\frac{i d \cdot \xi}{1+|x|^{2}}\right) \nu(d x) .
$$

Let $\psi: \mathbb{R}^{n} \rightarrow \mathbb{R}$ be a real valued continuous negative definite function. Then we have the representation

$$
\psi(\xi)=c+q(\xi)+\int_{\mathbb{R}^{n} \backslash\{0\}}(1-\cos (x \cdot \xi)) \nu(d x)
$$


where we denote by $\nu$ its Lévy measure which is not symmetric.

The following theorem is due to W.Hoh, [15], which relates the smoothness and the growth of $\psi$ to integrability properties of $\nu$.

Theorem 1.3.3. Let $\psi: \mathbb{R}^{n} \rightarrow \mathbb{R}$ be a continuous negative definite function with Lévy-Khinchin representation (1.5). Suppose that for $2 \leq l \leq m$ all absolute moments of the Lévy measure $\nu$ exist, i.e.

$$
M_{l}:=\int_{\mathbb{R}^{n} \backslash\{0\}}|x|^{l} \nu(d x)<\infty, 2 \leq l \leq m .
$$

Then, $\psi$ is of class $C^{m}\left(\mathbb{R}^{n}\right)$, and for $\alpha \in \mathbb{N}_{0}^{n},|\alpha| \leq m$, we have the estimates

$$
\left|\partial_{\xi}^{\alpha} \psi(\xi)\right| \leq c_{|\alpha|} \cdot \begin{cases}\psi(\xi), & \alpha=0, \\ \psi^{\frac{1}{2}}(\xi), & |\alpha|=1, \\ 1, & |\alpha| \geq 2,\end{cases}
$$

with $c_{0}=1, c_{1}=\left(2 M_{2}\right)^{\frac{1}{2}}+2 \lambda^{\frac{1}{2}}, c_{2}=M-2+2 \lambda$ and $c_{l}=M_{l}, 3 \leq l \leq m$, where $\lambda$ is the maximal eigenvalue of the quadratic form $q$ in (1.5).

The following examples of continuous negative definite functions are taken from [9] where the first example in particular will be useful with regard to further examples later in the thesis.

Example 1.3.4. For any $\alpha, \beta \in(0,1]$, the functions $\xi \mapsto|\xi|^{2 \alpha}$ and $\xi \mapsto|\xi|^{2 \alpha}+$ $|\xi|^{2 \beta}, \xi \in \mathbb{R}^{n}$, are continuous and negative definite.

Example 1.3.5. Fix any $\lambda \in(0,2)$ and choose $M=M(\lambda) \in \mathbb{N}$ such that $M>\frac{2}{(2-\lambda)}$. Then the measure

$$
\nu(d x):=\sum_{j=1}^{\infty} 2^{\lambda M^{j}-j} \varepsilon_{2^{-M^{j}}}(d x)
$$

is easily seen to be a Lévy measure. Therefore the function $\psi: \mathbb{R} \rightarrow \mathbb{R}$,

$$
\psi(\xi):=\int_{\mathbb{R}^{n} \backslash\{0\}}(1-\cos (x \cdot \xi)) \nu(d x)=\sum_{j=1}^{\infty} 2^{\lambda M^{j}-j}\left(1-\cos \left(2^{-M^{j}} \xi\right)\right),
$$

is continuous and negative definite. 
Example 1.3.6. Pick $0<\kappa<\lambda<2$ and denote by $\psi_{\lambda}(\xi)$ the function constructed in Example 1.3.5. Then

$$
\psi(\xi):=\psi_{\lambda}(\xi)+|\xi|^{\kappa}
$$

is a continuous negative function that oscillates for $|\xi| \rightarrow \infty$ between the curves $\xi \mapsto|\xi|^{\kappa}$ and $\xi \mapsto 2|\xi|^{\lambda}$. Moreover $\psi(\xi)=\mathcal{O}\left(|\xi|^{\lambda}\right)$ as $|\xi| \rightarrow \infty$.

\subsection{Bernstein Functions and Subordination}

Let us begin with

Definition 1.4.1. A real-valued function $f \in C^{\infty}((0, \infty))$ is said to be completely monotone if

$$
(-1)^{k} \frac{d^{k} f(x)}{d x^{k}} \geq 0
$$

holds for all $k \in \mathbb{N}$.

Definition 1.4.2. A real-valued function $f \in C^{\infty}((0, \infty))$ is called a Bernstein function if

$$
f \geq 0 \text { and }(-1)^{k} \frac{d^{k} f(x)}{d x^{k}} \leq 0
$$

holds for all $k \in \mathbb{N}$.

A Bernstein function is positive, increasing and concave and the set of all Bernstein functions forms a convex cone containing the positive constants. Bernstein functions are closely related to completely monotone functions, where the following theorem gives the link between these two classes of functions which is taken from [35].

Theorem 1.4.3. For a function $f:(0, \infty) \rightarrow \mathbb{R}$ the following two assertions are equivalent:

1) $f$ is a Bernstein function;

2) $f \geq 0$ and for all $t>0$ the function $x \mapsto e^{-t f(x)}$ is completely monotone. 
We will now look at an integral representation for Bernstein functions which is analogous to the Lévy-Khinchin formula, where the following theorem is taken from $[35]$.

Theorem 1.4.4. Let $f$ be a Bernstein function. Then there exist constants $a, b \geq 0$ and a measure $\mu$ on $(0, \infty)$ verifying

$$
\int_{0+}^{\infty} \frac{s}{1+s} \mu(d s)<\infty
$$

such that

$$
f(x)=a+b x+\int_{0+}^{\infty}\left(1-e^{-x s}\right) \mu(d s), x>0 .
$$

The triple $(a, b, \mu)$ is uniquely determined by $f$. Conversely, given $a, b \geq 0$ and a measure $\mu$ on $(0, \infty)$ satisfying (1.7), then (1.8) defines a Bernstein function.

A Borel measure $\mu$ is called a Radon measure if it is locally finite and inner regular. The support $\operatorname{supp} \mu$ of a Radon measure $\mu$ is the complement of the largest open set $G$ such that $\mu(G)=0$. Now, we want to relate Bernstein functions to certain semigroups of measures.

Definition 1.4.5. Let $\left(\eta_{t}\right)_{t \geq 0}$ be a convolution semigroup of measures on $\mathbb{R}$. It is said to be supported by $[0, \infty)$ if supp $\eta_{t} \subset[0, \infty)$ for all $t \geq 0$.

Convolution semigroups of measures $\left(\eta_{t}\right)_{t \geq 0}$ supported in $[0, \infty)$ are of particular interest and are better described by their Laplace transform $\mathcal{L}\left(\eta_{t}\right)$ than by their Fourier transform which is illustrated in the following theorem from [35].

Theorem 1.4.6. Let $f:(0, \infty) \rightarrow \mathbb{R}$ be a Bernstein function. Then there exists a unique convolution semigroup $\left(\eta_{t}\right)_{t \geq 0}$ supported by $[0, \infty)$ such that

$$
\mathcal{L}\left(\eta_{t}\right)(x)=\int_{0-}^{\infty} e^{-s x} \eta_{t}(d s)=e^{-t f(x)}, x>0 \text { and } t>0 .
$$

Conversely, for any convolution semigroup $\left(\eta_{t}\right)_{t \geq 0}$ supported by $[0, \infty)$ there exists a unique Bernstein function $f$ such that (1.9) holds.

Every Bernstein function $f$ extends to the half-plane $\operatorname{Re} z \geq 0$, and from this, we may state important properties of Bernstein functions and how they operate on negative definite functions. 
Lemma 1.4.7. For any Bernstein function $f$ and any continuous negative definite function $\psi: \mathbb{R}^{n} \rightarrow \mathbb{C}$, the function $f \circ \psi$ is also continuous and negative definite.

Let $\psi$ and $f$ to be the same as in Lemma 1.4.7. Since $f \circ \psi$ is a continuous negative definite function on $\mathbb{R}^{n}$, there exists a convolution semigroup $\left(\mu_{t}^{f}\right)_{t \geq 0}$ associated with $f \circ \psi$.

Proposition 1.4.8. Let $\psi \in C N\left(\mathbb{R}^{n}\right)$ be a continuous negative definite function with associated convolution semigroup $\left(\mu_{t}\right)_{t \geq 0}$ on $\mathbb{R}^{n}$. Further let $f$ be a Bernstein function with associated semigroup $\left(\eta_{t}\right)_{t \geq 0}$ supported on $[0, \infty)$. The convolution semigroup $\left(\mu_{t}^{f}\right)_{t \geq 0}$ on $\mathbb{R}^{n}$ associated with the continuous negative definite function $f \circ \psi$ is characterised by

$$
\int_{\mathbb{R}^{n}} \phi(x) \mu_{t}^{f}(d x)=\int_{0}^{\infty} \int_{\mathbb{R}^{n}} \phi(x) \mu_{s}(d x) \eta_{t}(d s), \phi \in C_{0}\left(\mathbb{R}^{n}\right) .
$$

Remark 1.4.9. Instead of (1.10) we will sometimes write

$$
\mu_{t}^{f}=\int_{0}^{\infty} \mu_{s} \eta_{t}(d s) \text { vaguely. }
$$

Definition 1.4.10. In the case of Proposition 1.4 .8 we call the convolution semigroup $\left(\mu_{t}^{f}\right)_{\geq 0}$ the semigroup subordinate (in the sense of Bochner) to $\left(\mu_{t}\right)_{t \geq 0}$ with respect to $\left(\eta_{t}\right)_{t \geq 0}$. The convolution semigroup $\left(\eta_{t}\right)_{t \geq 0}$ is sometimes called a subordinator.

Next, we will look at some examples of Bernstein functions and associated semigroups which is taken from [20].

Example 1.4.11. The function $x \mapsto a, a \geq 0$, is a Bernstein function, as is the funcion $x \mapsto b x, b \geq 0$. The associated semigroups are $\left(e^{-a t} \varepsilon_{0}\right)_{t \geq 0}$ and $\left(\varepsilon_{b t}\right)_{t \geq 0}$, respectively.

Example 1.4.12. If $s \geq 0$ the function $f(x)=1-e^{-x s}$ is a Bernstein function. It corresponds to the Poisson semigroup with jumps of size s, i.e.

$$
\eta_{t}=\sum_{k=0}^{\infty} e^{-t} \frac{t^{k}}{k !} \varepsilon_{s k}, \quad t \geq 0
$$


Example 1.4.13. The function $f(x)=\log (1+x)$ is a Bernstein function since

$$
\log (1+x)=\int_{0}^{\infty}\left(1-e^{-x s}\right) s^{-1} e^{-s} d s, x>0
$$

The semigroup associated with this Bernstein function is called the $\Gamma$-semigroup which is given by

$$
\eta_{t}=g_{t}(\cdot) \lambda^{(1)}
$$

where

$$
g_{t}(x)=\chi_{(0, \infty)}(x) \frac{1}{\Gamma(t)} x^{t-1} e^{-x} .
$$

Clearly, $x \mapsto \frac{1}{2} \log (1+x)$ is also a Bernstein function with corresponding convolution semigroup

$$
\eta_{t}(d s)=\chi_{(0, \infty)}(s) \frac{1}{\Gamma\left(\frac{t}{2}\right)} s^{\frac{t}{2}-1} e^{-s} \lambda^{(1)}(d s)
$$

We call this semigroup the modified $\Gamma$-semigroup.

Example 1.4.14. For any $\alpha \in[0,1]$ the function $f_{\alpha}(x)=x^{\alpha}$ is a Bernstein function. For $\alpha=0$ and $\alpha=1$ this is trivial but for $\alpha \in(0,1)$ we have

$$
x^{\alpha}=\frac{\alpha}{\Gamma(1-\alpha)} \int_{0}^{\infty}\left(1-e^{-x s}\right) s^{-\alpha-1} d s, x \geq 0 .
$$

The corresponding semigroup is called the one-sided stable semigroup of order $\alpha$ and is denoted by $\left(\sigma_{t}^{\alpha}\right)_{t \geq 0}$. For $\alpha=0$ we find $\sigma_{t}^{0}=e^{-t} \varepsilon_{0}$, and for $\alpha=1$ it follows that $\sigma_{t}^{1}=\varepsilon_{t}$. Only for $\alpha=\frac{1}{2}$ a closed expression of the density of $\sigma_{t}^{\alpha}$ is known where we have a formula for the density of $\sigma_{t}^{\frac{1}{2}}$ with respect to $\lambda^{(1)}$ :

$$
\sigma_{t}^{\frac{1}{2}}=g_{t}(\cdot) \lambda^{(1)}
$$

with

$$
g_{t}(s)=\chi_{(0, \infty)}(s) \frac{1}{\sqrt{4 \pi}} t s^{\frac{-3}{2}} e^{\frac{t^{2}}{4 s}}
$$

The following lemma is also taken from [20].

Lemma 1.4.15. A) For every Bernstein function $f$ we have

$$
\frac{f^{\prime}(s)}{f(s)} \leq \frac{1}{s} \text { for } s>0
$$


B) For every Bernstein function $f$ and all $c \geq 1$ it follows that

$$
\frac{1}{c} f(s) \leq f(c s) \leq c f(s)
$$

C) If $f$ is a complete Bernstein function, then

$$
\left|\frac{f^{(k+1)}(s)}{f^{(k)}(s)}\right| \leq \frac{k+1}{s}, s>0,
$$

holds for all $k \in \mathbb{N}$.

D) For the derivatives of any Bernstein function we have

$$
\left|f^{(k)}(s)\right| \leq \frac{k !}{s^{k}} f(s), \quad s>0 \text { and } k \in \mathbb{N}_{0} .
$$

Corollary 1.4.16. Let $f$ and $g$ be two Bernstein functions. Then $f \circ g$ is also a Bernstein function. 


\section{Chapter 2}

\section{Semigroups and Their}

\section{Generators}

\subsection{Results from Functional Analysis}

We begin with some general results from functional analysis. Firstly, we look at linear operators $A$ on topological vector spaces. Let $X, Y$ be two topological vector spaces. Recall that $(A, D(A))$ is a linear operator from $X$ to $Y$ if $D(A) \subset$ $X$ is a linear subspace and $A: D(A) \rightarrow Y$ is linear, where $D(A)$ is the domain of $A$. The following definitions are taken from [20].

Definition 2.1.1. Consider two linear operators $T$ and $S$ from $X$ into $Y$ with domains $D(T) \subset D(S)$. If $T u=S u$ for each $u \in D(T)$, then $S$ is called an extension of $T$ and we write $T \subset S$.

Definition 2.1.2. Let $(A, D(A))$ be a linear operator from $X$ to $Y$, both being topological vector spaces.

A) If $D(A) \subset X$ is dense we call $D(A)$ a dense subspace and $(A, D(A))$ densely defined.

B) We call $A$ a closed operator if the graph $\Gamma(A):=\{(u, A u) \mid u \in D(A)\}$ is closed in $X \times Y$. 
C) The operator $A$ is closable if it has a closed extension.

The smallest closed extension of $(A, D(A))$ is called its closure and denoted by $(\bar{A}, D(\bar{A}))$.

In the case of an operator acting between Banach spaces, $A^{*}$ stands for the conjugate operator, but in the case of an operator acting between Hilbert spaces, $A^{*}$ stands for the adjoint operator. For operators in a Hilbert space we have the following.

Definition 2.1.3. Let $(A, D(A))$ be a densely defined operator on a Hilbert space $\left(H,(\cdot, \cdot)_{H}\right)$.

A) The operator $A$ is called symmetric if $A^{*}$ is an extension of $A$.

B) We call $A$ self-adjoint if $A=A^{*}$.

For a symmetric operator we have $(A x, y)_{H}=(x, A y)_{H}$ for all $x, y \in D(A)$, whereas for self-adjoint operators we have in addition $D(A)=D\left(A^{*}\right)$.

Self-adjoint operators play a vital role in spectral theory and we indicate some results below, we follow from [33].

We denote $L(H)$ to be the space of bounded operators on a Hilbert space $H$. An important class of operators on Hilbert spaces is that of the projections.

Definition 2.1.4. If $P \in L(H)$ and $P^{2}=P$, then $P$ is called a projection. If in addition $P=P^{*}$, then $P$ is called an orthogonal projection.

The following definition is needed before discussing the spectral theorem.

Definition 2.1.5. Two Hilbert spaces $H_{1}$ and $H_{2}$ are said to be isomorphic if there is a linear operator $U$ from $H_{1}$ onto $H_{2}$ such that $(U x, U y)_{H_{2}}=(x, y)_{H_{1}}$ for all $x, y \in H_{1}$. Such an operator is called unitary.

In [33] a class of unbounded self-adjoint operators are discussed where the following result is given. 
Theorem 2.1.6. (Spectral theorem-multiplication operator form): Let $A$ be a self-adjoint operator on a Hilbert space $H$ with domain $D(A)$. Then there is a measure space $\langle M, \mu\rangle$ with $\mu$ a finite measure, a unitary operator $U: H \rightarrow$ $L^{2}(M, d \mu)$, and a real-valued function $f$ on $M$ which is finite a.e. so that

a) $\varphi \in D(A)$ if and only if $f(\cdot)(U \varphi)(\cdot) \in L^{2}(M, d \mu)$.

b) If $\varphi \in U(D(A))$, then $\left(U A U^{-1} \varphi\right)(m)=f(m) \varphi(m)$.

Using this theory we can define functions of a self-adjoint operator. Given a bounded Borel function $h$ on $\mathbb{R}$ we define

$$
h(A)=U^{-1} T_{h(f)} U
$$

where $T_{h(f)}$ is the operator on $L^{2}(M, d \mu)$ which acts by multiplication by the function $h(f(m))$. Therefore we obtain the following theorem given in [33].

Theorem 2.1.7. (Spectral theorem-functional calculus form): Let $A$ be a selfadjoint operator on $H$. Then there is a unique map $\hat{\phi}$ from the bounded Borel functions on $\mathbb{R}$ into $L(H)$ so that

a) $\hat{\phi}$ is an algebraic *-homomorphism, that is,

$$
\begin{gathered}
\phi(h g)=\phi(h) \phi(g) \phi(\lambda h)=\lambda \phi(h) \\
\phi(1)=I \phi(\bar{h})=\phi(h)^{*}
\end{gathered}
$$

b) $\hat{\phi}$ is norm continuous, that is, $\|\hat{\phi}(h)\|_{L(H)} \leq\|h\|_{\infty}$.

c) Let $h_{n}(x)$ be a sequence of bounded Borel functions with $h_{n}(x) \rightarrow x$ as $n \rightarrow \infty$ for $x$ and $\left|h_{n}(x)\right| \leq|x|$ for all $x$ and $n$. Then, for any $\varphi \in D(A)$, $\lim _{n \rightarrow \infty} \hat{\phi}\left(h_{n}\right) \varphi=A \varphi$.

d) If $h_{n}(x) \rightarrow h(x)$ pointwise and if the sequence $\left\|h_{n}\right\|_{\infty}$ is bounded, then $\hat{\phi}\left(h_{n}\right) \rightarrow \hat{\phi}(h)$ strongly. 
Functional calculus is useful in many cases. In particular the spectral theorem in its projection-valued measure form follows easily from the functional calculus. By definition given in [33] $P_{\Omega}$ is called the spectral projection of an unbounded self-adjoint operator $A$ where $\Omega$ is a Borel set of $\mathbb{R}$. The family of operators $\left\{P_{\Omega}\right\}$ is called a projection-valued measure with properties stated in the following proposition.

Proposition 2.1.8. The family $\left\{P_{\Omega}\right\}$ of spectral projections of unbounded selfadjoint operator, A, has the following properties:

a) Each $P_{\Omega}$ is an orthogonal projection;

b) $P_{\Omega}=0, P_{(-\infty, \infty)}=I$;

c) If $\Omega=\cup_{n=1} \Omega_{n}$ with $\Omega_{n} \cap \Omega_{m}=\emptyset$ if $n \neq m$, then $P_{\Omega}=s-\lim _{N \rightarrow \infty} \sum_{n=1}^{N} P_{\Omega_{n}}$;

d) $P_{\Omega_{1}} P_{\Omega_{2}}=P_{\Omega_{1} \cap \Omega_{2}}$.

For $\varphi \in H, \Omega \mapsto\left(\varphi, P_{\Omega} \varphi\right)$ is a well defined Borel measure $\nu$ on $\mathbb{R}$. If $P_{\Omega}$ is a projection-value measure, then $\left(\varphi, P_{\Omega} \varphi\right)$ is an ordinary measure for any $\varphi$. We will use the symbol $d\left(\varphi, P_{\lambda} \varphi\right)$ to mean integration with respect to this measure. Thus, given a bounded Borel function $g$ we can define $g(A)$, where $A$ is a selfadjoint operator, by

$$
(\varphi, g(A) \varphi)=\int_{-\infty}^{\infty} g(\lambda) d\left(\varphi, P_{\lambda} \varphi\right)
$$

Suppose $g$ is an unbounded complex-valued Borel function and let

$$
D_{g}=\left\{\left.\varphi\left|\int_{-\infty}^{\infty}\right| g(\lambda)\right|^{2} d\left(\varphi, P_{\lambda} \varphi\right)<\infty\right\} \text {. }
$$

Then, $D_{g}$ is dense in $H$ and an operator $g(A)$ is defined on $D_{g}$ by $(2.1)$.

We can write

$$
g(A)=\int g(\lambda) d P_{\lambda}
$$


In particular, for $\varphi, \psi \in D(A)$,

$$
(\varphi, A \psi)=\int_{-\infty}^{\infty} \lambda d\left(\varphi, P_{\lambda} \varphi\right)
$$

If $g$ is real-valued, then $g(A)$ is self-adjoint on $D_{g}$. We summarise these results in the following theorem.

Theorem 2.1.9. (Spectral theorem-projection valued measure form): There is a one-to-one correspondence between self-adjoint operators $(A, D(A))$ and projection-valued measures $\left\{P_{\Omega}\right\}$ on $H$, the correspondence being given by

$$
A=\int_{-\infty}^{\infty} \lambda d P_{\lambda}
$$

i.e. for all $\varphi \in D(A)$ we have $A \varphi=\int_{-\infty}^{\infty} \lambda d\left(\varphi, P_{\lambda} \varphi\right)$. If $g(\cdot)$ is a real-valued Borel function on $\mathbb{R}$, then

$$
g(A)=\int_{-\infty}^{\infty} g(\lambda) d P_{\lambda}
$$

defined on $D_{g}$ given by $(2.2)$ is self-adjoint.

If $A$ is unbounded and self-adjoint we can use the functional calculus to define $e^{i t A}$ and $e^{-t A}$.

Theorem 2.1.10. Let $A$ be a self-adjoint operator then we can use spectral calculus to define the unitary operator group

$$
e^{i t A}=\int_{-\infty}^{\infty} e^{i t \lambda} d P_{\lambda}
$$

and the contraction semigroup

$$
e^{-t A}=\int_{0}^{\infty} e^{-t \lambda} d P_{\lambda}
$$

We will now look at some results regarding quadratic forms, which can be found in detail in [33].

Definition 2.1.11. A quadratic form is a map $q: Q(q) \times Q(q) \rightarrow \mathbb{C}$, where $Q(q)$ is a dense linear subset of a Hilbert space $H$ called the form domain, such that $q(\cdot, \psi)$ is conjugate linear and $q(\varphi, \cdot)$ is linear for $\varphi, \psi \in Q(q)$. 
Moreover, if $q(\varphi, \psi)=\overline{q(\varphi, \psi)}$ we say that $q$ is symmetric. If $q(\varphi, \varphi) \geq 0$ for all $\varphi \in Q(q), q$ is called positive, and if $q(\varphi, \varphi) \geq-M\|\varphi\|^{2}$ for some $M$, we say that $q$ is semibounded. Additionally, if $q$ is semibounded, then it is automatically symmetric if $H$ is complex.

Recall that an operator $A$ is closed if and only if its graph is closed. Extending this notion to closed quadratic forms is defined as follows.

Definition 2.1.12. Let $q$ be a semibounded quadratic form, $q(\varphi, \varphi) \geq-M\|\varphi\|^{2}$. $q$ is called closed if $Q(q)$ is complete under the norm

$$
\|\varphi\|=\sqrt{q(\varphi, \varphi)+(M+1)\|\varphi\|^{2}} .
$$

The following theorem which characterizes $H^{*}$ is known as the Fréchet-Riesz theorem.

Theorem 2.1.13. Let $H$ be a Hilbert space. For each $T \in H^{*}$, there is an unique $y_{T} \in H$ such that $T(x)=\left(x, y_{T}\right)$ for all $x \in H$, where we also have $\left\|y_{T}\right\|_{H}=\|T\|_{H^{*}}$.

Every quadratic from defined in Definition 2.1.11 can be expressed in terms of a symmetric bilinear form. We define a bilinear form as follows.

Definition 2.1.14. Let $\left(H,(\cdot, \cdot)_{H}\right)$ be a Hilbert space. Further let $D(B) \subset H$ be a dense linear subspace. We call $B: D(B) \times D(B) \rightarrow \mathbb{C}$ a bilinear form on $H$ with domain $D(B)$ if for all $u, v, w \in D(B)$ and $\lambda, \mu \in \mathbb{C}$,

$$
B(\lambda u+\mu v, w)=\lambda B(u, w)+\mu B(v, w)
$$

and

$$
B(u, \lambda v+\mu w)=\bar{\lambda} B(u, v)+\bar{\mu} B(u, w)
$$

hold.

In order to discuss the form domain we need to discuss the spectral theorem for bounded operators which is taken from [33]. Firstly we need a definition. 
Definition 2.1.15. Let $(A, D(A))$ be a densely defined closed operator on $(X,\|\cdot\|)$, $R_{\lambda}=(\lambda-A)^{-1}$. The operator $R_{\lambda}$ is called the resolvent of $A$ at $\lambda$ if it is defined on $X$ and bounded. The set

$$
\begin{aligned}
\rho(A):= & \{\lambda \in \mathbb{C} \mid \lambda I-A \text { is surjective and has a continuous inverse defined on } \\
& R(\lambda-A)=X\}
\end{aligned}
$$

is called the resolvent set of $A$. If $\lambda \notin \rho(A)$, then $\lambda$ is said to be in the spectrum $\sigma(A)$ of $A$.

We are now in a position to state the following.

Theorem 2.1.16. Let $A$ be a bounded self-adjoint operator on $H$, a separable Hilbert space. Then, there exists measures $\left\{\mu_{n}\right\}_{n=1}^{N}(N=1,2, \ldots, \infty)$ on $\sigma(A)$ and a unitary operator

$$
U: H \rightarrow \oplus_{n=1}^{N} L^{2}\left(\mathbb{R}, \mu_{n}\right)
$$

so that

$$
\left(U A U^{-1} \psi\right)_{n}(\lambda)=\lambda \psi_{n}(\lambda)
$$

where we write an element $\psi \in \oplus_{n=1}^{N} L^{2}\left(\mathbb{R}, \mu_{n}\right)$ as an $N$-tuple $\left\langle\psi_{1}(\lambda), \ldots, \psi_{N}(\lambda)\right\rangle$. The realization of $A$ is called a spectral representation.

We will define the form domain of an operator through the following example.

Example 2.1.17. Let $A$ be a self-adjoint operator on a Hilbert space $H$. We pass to a spectral representation of $A$, so that $A$ is multiplication by $x$ on $\oplus_{n=1}^{N} L^{2}\left(\mathbb{R}, \mu_{n}\right)$. Let

$$
Q(q)=\left\{\left.\left\{\psi_{n}(x)\right\}_{n=1}^{N}\left|\sum_{n=1}^{N} \int_{-\infty}^{\infty}\right| x|| \psi_{n}(x)\right|^{2} d \mu_{n}<\infty\right\}
$$

and for $\psi, \varphi \in Q(q)$ define

$$
q(\varphi, \psi)=\sum_{n=1}^{N} \int_{-\infty}^{\infty} x \overline{\varphi_{n}(x)} \psi_{n}(x) d \mu_{n}
$$

We call $q$ the quadratic form associated with $A$ and write $Q(q)=Q(A)$, where $Q(A)$ is called the form domain of the operator $A$. 
We will find the Friedrichs' extension theorem useful which is taken from [34].

Theorem 2.1.18. Let $A$ be a positive symmetric operator and let $q(\varphi, \psi)=$ $(\varphi, A \psi)$ for $\varphi, \psi \in D(A)$. Then $q$ is a closable quadratic form and its closure $\hat{q}$ is the quadaratic form of an unique self-adjoint operator $\hat{A}$, where $\hat{A}$ is a positive extension of $A$. Moreover, $\hat{A}$ is the only self-adjoint extension of $A$ whose domain is contained in the form of $\hat{q}$.

\subsection{One-Parameter Semigroups}

We will now look at some theory on one-parameter semigroups which is taken from [20]. We begin by defining a sub-Markovian semigroup on $L^{p}\left(\mathbb{R}^{n}\right), 1 \leq p<$ $\infty$. Firstly, we have

Definition 2.2.1. A) A one parameter family $\left(T_{t}\right)_{t \geq 0}$ of bounded linear operators $T_{t}: X \rightarrow X$ on a Banach space $\left(X,\|\cdot\|_{X}\right)$ is called a (one parameter) semigroup of linear operators, if $T_{0}=i d$ and $T_{s+t}=T_{s} \circ T_{t}$ hold for all $s, t \geq 0$.

B) We call $\left(T_{t}\right)_{t \geq 0}$ strongly continuous if

$$
\lim _{t \rightarrow 0}\left\|T_{t} u-u\right\|_{X}=0
$$

for all $u \in X$.

C) The semigroup $\left(T_{t}\right)_{t \geq 0}$ is called a contraction semigroup, if for all $t \geq 0$

$$
\left\|T_{t}\right\| \leq 1
$$

holds, i.e. if each of the operators $T_{t}$ is a contraction.

Definition 2.2.2. Let $\left(T_{t}\right)_{t \geq 0}$ be a strongly continuous contraction semigroup on $L^{p}\left(\mathbb{R}^{n}\right), 1 \leq p<\infty$. We call $\left(T_{t}\right)_{t \geq 0}$ a sub-Markovian semigroup on $L^{p}$, $1 \leq p<\infty$, if for $u \in L^{p}\left(\mathbb{R}^{n}\right)$ such that $0 \leq u \leq 1$ almost everywhere it follows that $0 \leq T_{t} u \leq 1$ almost everywhere. 
Here, we have defined a $L^{p}$ sub-Markovian semigroup. However, we are mainly interested in $L^{2}$ sub-Markovian semigroups.

We will now state an important definition in the theory of one parameter semigroups of operators which is that of a generator.

Definition 2.2.3. Let $\left(T_{t}\right)_{t \geq 0}$ be a strongly continuous semigroup of operators on a Banach space $\left(X,\|\cdot\|_{X}\right)$. The generator $A$ of $\left(T_{t}\right)_{t \geq 0}$ is defined by

$$
A u:=\lim _{t \rightarrow 0} \frac{T_{t} u-u}{t} \text { (strong limit) }
$$

with domain

$$
D(A):=\left\{u \in X \mid \lim _{t \rightarrow 0} \frac{T_{t} u-u}{t} \text { exists as strong limit }\right\} .
$$

We look at further results from the general theory of semigroups where the following lemma taken from [20] discusses the relation of $A$ and $\left(T_{t}\right)_{t \geq 0}$.

Lemma 2.2.4. Let $\left(T_{t}\right)_{t \geq 0}$ be a strongly continuous semigroup on the Banach space $\left(X,\|\cdot\|_{X}\right)$ and denote by $A$ its generator with domain $D(A) \subset X$.

A. For any $u \in X$ and $t \geq 0$ it follows that $\int_{0}^{t} T_{s} u d s \in D(A)$ and

$$
T_{t} u-u=A \int_{0}^{t} T_{s} u d s .
$$

B. For $u \in D(A)$ and $t \geq 0$ we have $T_{t} u \in D(A)$, i.e. $D(A)$ is invariant under $T_{t}$, and

$$
\frac{d}{d t} T_{t} u=A T_{t} u=T_{t} A u
$$

$C$. For $u \in D(A)$ and $t \geq 0$ we always get

$$
T_{t} u-u=\int_{0}^{t} A T_{s} u d s=\int_{0}^{t} T_{s} A u d s
$$

Lemma 2.2.5. Let $\left(T_{t}\right)_{t \geq 0}$ be a strongly continuous semigroup on $\left(X,\|\cdot\|_{X}\right)$. Then there exists constants $w \geq 0$ and $M_{w} \geq 1$ such that

$$
\left\|T_{t}\right\| \leq M_{w} e^{w t} .
$$


We will introduce the Hille-Yosida theorem which allows us to characterise generators of strongly continuous contraction semigroups. Firstly, we will state some definitions.

Definition 2.2.6. A linear operator $A: D(A) \rightarrow X, D(A) \subset X$, is called dissipative if

$$
\|\lambda u-A u\|_{X} \geq \lambda\|u\|_{X}
$$

holds for all $\lambda>0, u \in D(A)$.

Definition 2.2.7. Let $A: D(A) \rightarrow B\left(\mathbb{R}^{n} ; \mathbb{R}\right)$ be a linear operator, where $B\left(\mathbb{R}^{n}\right)$ is a Borel measurable function, $D(A) \subset B\left(\mathbb{R}^{n} ; \mathbb{R}\right)$. We say that $(A, D(A))$ satisfies the positive maximum principle if for any $u \in D(A)$ such that for some $x_{0} \in \mathbb{R}^{n}, u\left(x_{0}\right)=\sup _{x \in \mathbb{R}^{n}} u(x) \geq 0$ it follows that $A u\left(x_{0}\right) \leq 0$.

Lemma 2.2.8. Suppose that a linear operator $(A, D(A)), D(A) \subset C_{\infty}\left(\mathbb{R}^{n} ; \mathbb{R}\right)$, on $C_{\infty}\left(\mathbb{R}^{n} ; \mathbb{R}\right)$ satisfies the positive maximum principle on $D(A)$. Then $A$ is dissipative.

Definition 2.2.9. Let $\left(T_{t}\right)_{t \geq 0}$ be a strongly continuous contraction semigroup on $\left(C_{\infty}\left(\mathbb{R}^{n} ; \mathbb{R}\right),\|\cdot\|_{\infty}\right)$ which is positivity preserving. Then $\left(T_{t}\right)_{t \geq 0}$ is called a Feller semigroup.

We can now formulate the Hille-Yosida-Ray theorem which is taken from [20].

Theorem 2.2.10. A linear operator $(A, D(A)), D(A) \subset C_{\infty}\left(\mathbb{R}^{n}\right)$, on $C_{\infty}\left(\mathbb{R}^{n}\right)$ is closable and its closure is the generator of a Feller semigroup if and only if the following conditions hold

1. $D(A) \subset C_{\infty}\left(\mathbb{R}^{n}\right)$ is dense;

2. A satisfies the positive maximum principle;

3. $R(\lambda-A)$ is dense in $C_{\infty}\left(\mathbb{R}^{n}\right)$ for some $\lambda>0$. 


\subsection{Dirichlet Spaces}

We begin by looking at some general results involving Dirichlet spaces, where the following definition is taken from [30], see also [11].

Definition 2.3.1. A closed form $(\mathcal{E}, D(\mathcal{E}))$ on $L^{2}\left(\mathbb{R}^{n}\right)$ is called a Dirichlet form if for all $u \in D(\mathcal{E})$ it follows that $u^{+} \wedge 1 \in D(\mathcal{E})$ and

$$
\begin{aligned}
& \mathcal{E}\left(u+\left(u^{+} \wedge 1\right), u-\left(u^{+} \wedge 1\right)\right) \geq 0, \\
& \mathcal{E}\left(u-\left(u^{+} \wedge 1\right), u+\left(u^{+} \wedge 1\right)\right) \geq 0 .
\end{aligned}
$$

If $(\mathcal{E}, D(\mathcal{E}))$ is in addition symmetric, in which case we have

$$
\mathcal{E}\left(u^{+} \wedge 1, u^{+} \wedge 1\right) \leq \mathcal{E}(u, u)
$$

it is called a symmetric Dirichlet form.

The notion of a symmetric Dirichlet form was introduced in [3] and [4].

The translation invariant symmetric Dirichlet forms are given by

$$
\mathcal{E}^{\psi}(u, v)=\int_{\mathbb{R}^{n}} \psi(\xi) \hat{u}(\xi) \overline{\hat{v}(\xi)} d \xi, u, v \in \mathcal{S}\left(\mathbb{R}^{n}\right),
$$

where $\psi: \mathbb{R}^{n} \rightarrow \mathbb{R}$ is a continuous negative definite function, see [9].

The domain $\mathcal{F}^{\psi}$ of $\mathcal{E}^{\psi}$ is given by the Hilbert spaces $H^{\psi, 1}\left(\mathbb{R}^{n}\right)$ where for $s \geq 0$, $H^{\psi, s}\left(\mathbb{R}^{n}\right)$ is defined as follows

$$
H^{\psi, s}\left(\mathbb{R}^{n}\right):=\left\{u \in L^{2}\left(\mathbb{R}^{n}\right): \int_{\mathbb{R}^{n}}(1+\psi(\xi))^{s}|\hat{u}(\xi)|^{2} d \xi<\infty\right\} .
$$

Note, that $\mathcal{S}\left(\mathbb{R}^{n}\right)$ is a dense subset of $H^{\psi, s}$. Moreover, we can associate with $\left(\mathcal{F}^{\psi}, \mathcal{E}^{\psi}\right)$ the operator semigroup $\left(T_{t}\right)_{t \geq 0}$ on $L_{2}\left(\mathbb{R}^{n}\right)$ defined by

$$
T_{t} u(x)=(2 \pi)^{\frac{-n}{2}} \int_{\mathbb{R}^{n}} e^{i x \cdot \xi} e^{-t \psi(\xi)} \hat{u}(\xi) d \xi=\int_{\mathbb{R}^{n}} u(x-y) \mu_{t}(d y),
$$

where $\left(\mu_{t}\right)_{t \geq 0}$ is the vaguely continuous convolution semigroup of sub-probability measures on $\mathbb{R}^{n}$ such that

$$
\hat{\mu}_{t}(\xi)=(2 \pi)^{\frac{-n}{2}} e^{-t \psi(\xi)}
$$


It can be shown that the one parameter family $\left(T_{t}\right)_{t \geq 0}$ of bounded linear operators $T_{t}: L^{2}\left(\mathbb{R}^{n}\right) \rightarrow L^{2}\left(\mathbb{R}^{n}\right)$ gives a strongly continuous one-parameter semigroup on $L^{2}\left(\mathbb{R}^{n}\right)$, where we find the following example in [20].

Example 2.3.2. Let $\left(\mu_{t}\right)_{t \geq 0}$ ba a convolution semigroup on $\mathbb{R}^{n}$. For $u \in \mathcal{S}\left(\mathbb{R}^{n}\right)$ we define

$$
T_{t} u(x)=\int_{\mathbb{R}^{n}} u(x-y) \mu_{t}(d y)
$$

and find with a continuous negative definite function $\psi: \mathbb{R}^{n} \rightarrow \mathbb{C}$ that

$$
\left(T_{t} u\right)^{\wedge}(\xi)=e^{-t \psi(\xi)} \hat{u}(\xi)
$$

We find

$$
\begin{aligned}
\left\|T_{t} u-u\right\|_{0}^{2} & =\int_{\mathbb{R}^{n}}\left|e^{-t \psi(\xi)} \hat{u}(\xi)-\hat{u}(\xi)\right|^{2} d \xi \\
& =\int_{\mathbb{R}^{n}}\left|e^{-t \psi(\xi)}-1\right|^{2}|\hat{u}(\xi)|^{2} d \xi
\end{aligned}
$$

implying the strong continuity of $\left(T_{t}\right)_{t \geq 0}$ as $t$ tends to 0. From (2.3) we can see that $\left(T_{t}\right)_{t \geq 0}$ is a semigroup. Hence, $\left(T_{t}\right)_{t \geq 0}$ extends to a strongly continuous semigroup on $L^{2}\left(\mathbb{R}^{n}\right)$.

We now look at some estimates involving the Dirichlet forms.

Lemma 2.3.3. Given the translation invariant symmetric Dirichet form defined by

$$
\mathcal{E}^{\psi}(u, v)=\int_{\mathbb{R}^{n}} \psi(\xi) \hat{u}(\xi) \overline{\hat{v}(\xi)} d \xi
$$

where $\psi(\xi)$ is real-valued. Then for every $u, v \in H^{\psi, 1}$, the following estimate holds

$$
\left|\mathcal{E}^{\psi}(u, v)\right| \leq\|u\|_{\psi, 1}\|v\|_{\psi, 1} .
$$


Proof. For $u, v \in \mathcal{S}\left(\mathbb{R}^{n}\right)$,

$$
\begin{aligned}
\left|\mathcal{E}^{\psi}(u, v)\right| & =\left|\int_{\mathbb{R}^{n}} \psi(\xi) \hat{u}(\xi) \overline{\hat{v}(\xi)} d \xi\right| \\
& \leq \int_{\mathbb{R}^{n}}|\psi(\xi)||\hat{u}(\xi)||\hat{v}(\xi)| d \xi \\
& \leq\left(\left(\int_{\mathbb{R}^{n}} \psi(\xi)^{\frac{1}{2}}|\hat{u}(\xi)|\right)^{2} d \xi\right)^{\frac{1}{2}}\left(\left(\int_{\mathbb{R}^{n}} \psi(\xi)^{\frac{1}{2}}|\hat{v}(\xi)|\right)^{2} d \xi\right)^{\frac{1}{2}} \\
& =\left(\int_{\mathbb{R}^{n}} \psi(\xi)|\hat{u}(\xi)|^{2} d \xi\right)^{\frac{1}{2}}\left(\int_{\mathbb{R}^{n}} \psi(\xi)|\hat{v}(\xi)|^{2} d \xi\right)^{\frac{1}{2}} \\
& \leq\left(\int_{\mathbb{R}^{n}}(1+\psi(\xi))|\hat{u}(\xi)|^{2} d \xi\right)^{\frac{1}{2}}\left(\int_{\mathbb{R}^{n}}(1+\psi(\xi))|\hat{v}(\xi)|^{2} d \xi\right)^{\frac{1}{2}} \\
& =\|u\|_{\psi, 1}\|v\|_{\psi, 1}
\end{aligned}
$$

where we used that $\psi(\xi) \leq c_{\psi}(1+\psi(\xi))$, making use of the fact that a real-valued continuous negative definite function is non-negative.

We want to get a better understanding of Example 2.3.2 in relation to Lemma 2.3.3. For this let $u, v \in \mathcal{S}\left(\mathbb{R}^{n}\right)$ and consider

$$
\left(\frac{T_{t} u-u}{t}, v\right)_{L^{2}}=\left(\frac{e^{-t \psi(\cdot)} \hat{u}-\hat{u}}{t}, \hat{v}\right)_{L^{2}}
$$

which follows from Plancherel's theorem. Passing on the right hand side to the limit $t \rightarrow 0$ we obtain, assuming for simplicity $\psi(\xi) \in \mathbb{R}$,

$$
\lim _{t \rightarrow 0}\left(\frac{e^{-t \psi(\cdot)} \hat{u}-\hat{u}}{t}, \hat{v}\right)_{L^{2}}=(-\psi(\cdot) \hat{u}, \hat{v})_{L^{2}}
$$

or

$$
\lim _{t \rightarrow 0}\left(-\left(\frac{e^{-t \psi(\cdot)} \hat{u}-\hat{u}}{t}, \hat{v}\right)_{L^{2}}=(\psi(\cdot) \hat{u}, \hat{v})_{L^{2}}=\mathcal{E}^{\psi}(u, v)\right.
$$

for all $u, v \in \mathcal{S}\left(\mathbb{R}^{n}\right)$. For this calculation we have used the estimate

$$
\left|\frac{e^{-a t}-1+a t}{t}\right| \leq t \frac{a^{2}}{2}, a>0
$$

and the growth bound $|\psi(\xi)| \leq c_{\psi}\left(1+|\xi|^{2}\right)$.

Thus for $u, v \in \mathcal{S}\left(\mathbb{R}^{n}\right)$ we find

$$
\begin{aligned}
\lim _{t \rightarrow 0}\left(\frac{T_{t} u-u}{t}, v\right)_{L^{2}} & =\lim _{t \rightarrow 0}\left(\frac{e^{-t \psi(\cdot)} \hat{u}-\hat{u}}{t}, \hat{v}\right)_{L^{2}} \\
& =(-\psi(\cdot) \hat{u}, \hat{v})_{L^{2}} .
\end{aligned}
$$


With $A u:-\psi(D) u, u \in \mathcal{S}\left(\mathbb{R}^{n}\right)$ it follows

$$
\lim _{t \rightarrow 0}\left(\frac{T_{t} u-u}{t}, v\right)_{L^{2}}=(A u, v)_{L^{2}} .
$$

In Chapter 5 we will pick up the ideas outlined in this paragraph and will discuss as a central topic extended Dirichlet spaces.

Furthermore, we have

Lemma 2.3.4. Given the Dirichlet form, $\mathcal{E}^{\psi}$, we define

$$
\mathcal{E}_{\lambda_{0}}^{\psi}(u, v)=\mathcal{E}^{\psi}(u, v)+\lambda_{0}(u, v)_{L^{2}}
$$

where we consider $\lambda_{0}>0$ and $\psi(\xi)$ is real-valued. Then for every $u, v \in H^{\psi, 1}$ the following estimate holds

$$
\left|\mathcal{E}_{\lambda_{0}}^{\psi}(u, v)\right| \leq c\left(\lambda_{0}\right)\|u\|_{\psi, 1}\|v\|_{\psi, 1} .
$$

Proof. For every $u, v \in \mathcal{S}\left(\mathbb{R}^{n}\right)$,

$$
\begin{aligned}
\left|\mathcal{E}_{\lambda_{0}}^{\psi}(u, v)\right| & =\left|\int_{\mathbb{R}^{n}} \psi(\xi) \hat{u}(\xi) \overline{\hat{v}(\xi)}+\int_{\mathbb{R}^{n}} \lambda_{0} \hat{u}(\xi) \overline{\hat{v}(\xi)} d \xi\right| \\
& \leq \int_{\mathbb{R}^{n}}(1+\psi(\xi))|\hat{u}(\xi)||\hat{v}(\xi)| d \xi+\left|\lambda_{0}\right| \int_{\mathbb{R}^{n}}|\hat{u}(\xi)||\hat{v}(\xi)| d \xi \\
& =\|u\|_{\psi, 1}\|v\|_{\psi, 1}+\left|\lambda_{0}\right|\|u\|_{0}\|v\|_{0} \\
& \leq c\left(\lambda_{0}\right)\|u\|_{\psi, 1}\|v\|_{\psi, 1} .
\end{aligned}
$$

There is a one-to-one correspondence between Dirichlet forms and strongly continuous symmetric semigroups $\left(T_{t}\right)_{t \geq 0}$ on $L^{2}$. In order to illustrate this in a theorem, we need to define a Dirichlet operator which we find in [20]. Firstly, we have

Lemma 2.3.5. Let $\left(T_{t}\right)_{t \geq 0}$ be a sub-Markovian semigroup on $L^{p}\left(\mathbb{R}^{n}\right), 1<p<$ $\infty$, with generator $(A, D(A))$. Then for all $u \in D(A)$ we have

$$
\int_{\mathbb{R}^{n}}(A u)\left((u-1)^{+}\right)^{p-1} d x \leq 0 .
$$


Definition 2.3.6. A closed, densely defined linear operator $A: D(A) \rightarrow L^{p}\left(\mathbb{R}^{n}\right)$, $1<p<\infty, D(A) \subset L^{p}\left(\mathbb{R}^{n}\right)$, is called a Dirichlet operator if for all $u \in D(A)$ the relation (2.4) holds.

The following result is taken from both [20] and [30].

Proposition 2.3.7. Suppose that a Dirichlet operator $(A, D(A))$ on $L^{p}\left(\mathbb{R}^{n} ; \mathbb{R}\right)$, $1<p<\infty$, generates a strongly continuous contraction semigroup $\left(T_{t}\right)_{t \geq 0}$ on $L^{p}\left(\mathbb{R}^{n} ; \mathbb{R}\right)$ with corresponding resolvent $\left(R_{\lambda}\right)_{\lambda>0}$. Then $\left(T_{t}\right)_{t \geq 0}$ and $\left(R_{\lambda}\right)_{\lambda>0}$ are sub-Markovian. Moreover, we can state that the following are equivalent.

i) $\left(R_{\lambda}\right)_{\lambda>0}$ is sub-Markovian.

ii) $\left(T_{t}\right)_{t \geq 0}$ is sub-Markovian.

iii) $(A, D(A))$ is a Dirichlet operator.

We can now deduce that the following theorem holds.

Theorem 2.3.8. Let $(A, D(A))$ be a Dirichlet operator on $L^{2}\left(\mathbb{R}^{n}\right)$ which generates a sub-Markovian semigroup $\left(T_{t}\right)_{t \geq 0}$. If $(A, D(A))$ is self-adjoint, then $(\mathcal{E}, D(\mathcal{E}))$ is a symmetric Dirichlet form. Conversely, for a symmetric Dirichlet form it follows that $(A, D(A))$ is a self-adjoint operator. Moreover the semigroup has an analytic extension to $L^{2}\left(\mathbb{R}^{n}\right)$.

If we combine Theorem 2.3.8 with the calculation following Lemma 2.3.3 we see that at least on $\mathcal{S}\left(\mathbb{R}^{n}\right)$ we have for the generator of $\left(T_{t}^{\psi}\right)_{t \geq 0}$, where $\psi: \mathbb{R}^{n} \rightarrow \mathbb{R}$ and $\left(T_{t}^{\psi}\right)_{t \geq 0}$ denotes the semigroup from Example 2.3.2, the formula

$$
A^{\psi} u(x)=-\psi(D) u(x)=(2 \pi)^{-\frac{n}{2}} \int_{\mathbb{R}^{n}} e^{i x \xi}(-\psi(\xi)) \hat{u}(\xi) d \xi .
$$

The following results will be helpful when discussing extended Dirichlet spaces which we will do later on.

Lemma 2.3.9. Let $(\mathcal{E}, D(\mathcal{E}))$ be a symmetric Dirichlet form. Then every normal contraction operates on $(\mathcal{E}, D(\mathcal{E}))$, i.e. if $T: \mathbb{R} \rightarrow \mathbb{R}$ such that $T(0)=0$ and 
$|T(s)-T(t)| \leq|s-t|$ then $T \circ u \in D(\mathcal{E})$ for every $u \in D(\mathcal{E})$ and $\mathcal{E}(T \circ u, T \circ u) \leq$ $\mathcal{E}(u, u)$.

\subsection{Pseudo-Differential Operators with Nega- tive Definite Symbols}

In this section we look at some results with regard to the pseudo-differential operator with negative definite symbols. A linear operator $A: C_{0}^{\infty}\left(\mathbb{R}^{n}\right) \rightarrow C(\mathbb{R})$ which satisfies the positive maximum principle, has a representation as a pseudodifferential operator

$$
A u(x)=q(x, D) u(x)=(2 \pi)^{\frac{-n}{2}} \int_{\mathbb{R}^{n}} e^{i x \cdot \xi} q(x, \xi) \hat{u}(\xi) d \xi,
$$

where we assume that $q: \mathbb{R}^{n} \times \mathbb{R}^{n} \rightarrow \mathbb{C}$ is a locally bounded funtion such that for every $x \in \mathbb{R}^{n}$ the function $q(x, \cdot): \mathbb{R}^{n} \rightarrow \mathbb{C}$ is a negative definite and continuous. The following definition and assumptions are taken from [21].

Definition 2.4.1. We call a function $q: \mathbb{R}^{n} \times \mathbb{R}^{n} \rightarrow \mathbb{C}$ a (continuous) negative definite symbol if $q$ is locally bounded (continuous) and for each $x \in \mathbb{R}^{n}$ the function $q(x, \cdot): \mathbb{R}^{n} \rightarrow \mathbb{C}$ is negative definite and continuous.

For a negative definite symbol $q$ for every compact set $K \subset \mathbb{R}^{n}$ there exists a constant $c_{K}$ such that

$$
|q(x, \xi)| \leq c_{K}\left(1+|\xi|^{2}\right)
$$

holds for all $x \in K$ and $\xi \in \mathbb{R}^{n}$.

For a (negative definite) symbol $q(x, \xi)$ we set

$$
\hat{q}(\eta, \xi):=(2 \pi)^{\frac{-n}{2}} \int_{\mathbb{R}^{n}} e^{-i x \cdot \eta} q(x, \xi) d x=F_{x \rightarrow \eta}(q(x, \xi))(\eta),
$$

whenever this Fourier transform exists.

We will also consider the following decomposition of the symbol $q(x, \xi)$

$$
q(x, \xi)=q\left(x_{0}, \xi\right)+\left(q(x, \xi)-q\left(x_{0}, \xi\right)\right)=q_{1}(\xi)+q_{2}(x, \xi)
$$


which we obtain when freezing the coefficients with the grid point $x_{0}$.

Before moving on we will state some assumptions which will be essential in the following.

Assumption 2.4.2. We assume that the function $q: \mathbb{R}^{n} \times \mathbb{R}^{n} \rightarrow \mathbb{C}$ is a continuous negative definite symbol having the decomposition (2.5) into a continuous negative definite function $q_{1}: \mathbb{R}^{n} \rightarrow \mathbb{C}$ and a continuous function $q_{2}: \mathbb{R}^{n} \times \mathbb{R}^{n} \rightarrow \mathbb{C}$

A.1. The function $q_{1}$ is continuous and negative definite and satisfies the following

$$
\frac{1}{\tau_{1}}(1+\psi(\xi)) \leq 1+\left|q_{1}(\xi)\right| \leq \tau_{1}(1+\psi(\xi))
$$

for some constant $\tau_{1}>0$ and a fixed continuous negative definite function $\psi(\xi)$ such that for large $|\xi|, \xi \in \mathbb{R}^{n}, \psi(\xi) \geq c|\xi|^{r_{0}}$ holds with some constants $c>0$, $r_{0}>0$.

A.2. For $m \in \mathbb{N}_{0}$ the function $x \mapsto q_{2}(x, \xi)$ belongs to $C^{m}\left(\mathbb{R}^{n}\right)$ and we have the estimate

$$
\left|\partial_{x}^{\alpha} q_{2}(x, \xi)\right| \leq \varphi_{\alpha}(x)(1+\psi(\xi))
$$

for all $\alpha \in \mathbb{N}_{0}^{n},|\alpha| \leq m$, with function $\varphi_{\alpha} \in L^{1}\left(\mathbb{R}^{n}\right)$. Moreover, we have for all $k \in \mathbb{N}_{0},|k| \leq m$, the estimate

$$
|\hat{q}(\eta, \xi)| \leq \tilde{\gamma}_{k, n} \sum_{|\alpha| \leq m}\left\|\varphi_{\alpha}\right\|_{L^{1}}\left(1+|\eta|^{2}\right)^{\frac{-m}{2}}(1+\psi(\xi)) .
$$

We will now look at the bilinear form in conjunction with the operator $q(x, D)$, with [21] being our main reference.

We introduce the bilinear form

$$
B(u, v):=(q(x, D) u, v)_{0}
$$

which is assosciated to $q(x, D)$ and defined on $C_{0}^{\infty}\left(\mathbb{R}^{n}\right)$ where we have the decomposition

$$
B(u, v)=B^{q_{1}}(u, v)+B^{q_{2}}(u, v),
$$


with $B^{q_{1}}(u, v):=\left(q_{1}(D) u, v\right)_{0}$ and $B^{q_{2}}(u, v):=\left(q_{2}(x, D) u, v\right)_{0}$.

Note, that since $\mathcal{S}\left(\mathbb{R}^{n}\right)$ is a dense subset of $H^{\psi, s}$, we can prove the following estimates for $u, v \in \mathcal{S}\left(\mathbb{R}^{n}\right)$, and then conclude that they hold first in $H^{\psi, s}$.

Theorem 2.4.3. Suppose that $q_{1}(\xi)$ and $q_{2}(x, \xi)$ satisfy $A .1$ and $A .2$ with $m \geq$ $n+2$. Then $B$ is continuous on $H^{\psi, 1}\left(\mathbb{R}^{n}\right)$, i.e. for all $u, v \in H^{\psi, 1}\left(\mathbb{R}^{n}\right)$ we have

$$
|B(u, v)| \leq c\|u\|_{\psi, 1}\|v\|_{\psi, 1}
$$

Proof. Firstly, we show that

$$
\left|B^{q_{1}}(u, v)\right| \leq \tau_{1}\|u\|_{\psi, 1}\|v\|_{\psi, 1}
$$

with $\tau_{1}$ as in (2.6). To do this it is sufficient to look at the case for all $u, v \in$ $\mathcal{S}\left(\mathbb{R}^{n}\right)$. From (2.6) we obtain

$$
\begin{aligned}
\left|B^{q_{1}}(u, v)\right| & =\left|\int_{\mathbb{R}^{n}} q_{1}(\xi) \hat{u}(\xi) \overline{\hat{v}(\xi)} d \xi\right| \\
& \leq \tau_{1} \int_{\mathbb{R}^{n}}(1+\psi(\xi))|\hat{u}(\xi) \| \hat{v}(\xi)| d \xi \\
& \leq \tau_{1}\|u\|_{\psi, 1}\|v\|_{\psi, 1} .
\end{aligned}
$$

For the second half of the proof it remains to show that

$$
\left|B^{q_{2}}(u, v)\right| \leq \kappa_{2} \sum_{|\alpha| \leq n+2}\left\|\varphi_{\alpha}\right\|_{L^{1}}\|u\|_{\psi, 1}\|v\|_{\psi, 1} .
$$


For all $u, v \in \mathcal{S}\left(\mathbb{R}^{n}\right)$ we deduce

$$
\begin{aligned}
& \left|B^{q_{2}}(u, v)\right|=\left|\left(q_{2}(x, D) u, v\right)_{0}\right| \\
& =\left|\int_{\mathbb{R}^{n}} q_{2}(x, D) u(x) v(x) d x\right| \\
& =\left|\int_{\mathbb{R}^{n}} \int_{\mathbb{R}^{n}} \hat{q}_{2}(\xi-\eta, \eta) \hat{u}(\eta) \overline{\hat{v}(\xi)} d \eta d \xi\right| \\
& \leq \tilde{\gamma}_{n+2, n} \sum_{|\alpha| \leq n+2}\left\|\varphi_{\alpha}\right\|_{L^{1}} \int_{\mathbb{R}^{n}} \int_{\mathbb{R}^{n}}\left(1+|\xi-\eta|^{2}\right)^{\frac{-(n+2)}{2}} \\
& \times(1+\psi(\eta))|\hat{u}(\eta)||\hat{v}(\xi)| d \eta d \xi \\
& \leq \tilde{\gamma}_{n+2, n} \sum_{|\alpha| \leq n+2}\left\|\varphi_{\alpha}\right\|_{L^{1}} \int_{\mathbb{R}^{n}} \int_{\mathbb{R}^{n}}\left(1+|\xi-\eta|^{2}\right)^{\frac{-(n+2)}{2}}\left(\frac{1+\psi(\eta)}{1+\psi(\xi)}\right)^{\frac{1}{2}} \\
& \times(1+\psi(\eta))^{\frac{1}{2}}|\hat{u}(\eta)|(1+\psi(\xi))^{\frac{1}{2}}|\hat{v}(\xi)| d \eta d \xi \\
& \leq \sqrt{2} \tilde{\gamma}_{n+2, n}\left(1 \vee c_{\psi}\right)^{\frac{1}{2}} \sum_{|\alpha| \leq n+2}\left\|\varphi_{\alpha}\right\|_{L^{1}} \int_{\mathbb{R}^{n}} \int_{\mathbb{R}^{n}}\left(1+|\xi-\eta|^{2}\right)^{\frac{-(n+2)}{2}} \\
& \times(1+\psi(\eta))^{\frac{1}{2}}|\hat{u}(\eta)|(1+\psi(\xi))^{\frac{1}{2}}|\hat{v}(\xi)| d \eta d \xi \\
& \leq \kappa_{2} \sum_{|\alpha| \leq n+2}\left\|\varphi_{\alpha}\right\|_{L^{1}}\|u\|_{\psi, 1}\|v\|_{\psi, 1}
\end{aligned}
$$

with

$$
\kappa_{2}=\sqrt{2}\left(1 \vee c_{\psi}\right)^{\frac{1}{2}} \tilde{\gamma}_{n+2, n} \tilde{c}_{n, n+1}
$$

This implies (2.7).

Theorem 2.4.4. Suppose that $q_{1}(\xi)$ and $q_{2}(x, \xi)$ satisify $A .1$ and $A .2$ with $m \geq$ $n+2$. Assume further with $\kappa_{2}$ from (2.8) that

$$
\delta_{1}:=\gamma_{0}-\kappa_{2} \sum_{|\alpha| \leq n+2}\left\|\varphi_{\alpha}\right\|_{L^{1}}>0
$$

Then we have for all $u \in H^{\psi, 1}\left(\mathbb{R}^{n}\right)$

$$
|B(u, u)| \geq \operatorname{ReB}(u, u) \geq \delta_{1}\|u\|_{\psi, 1}^{2}-\tilde{\lambda}_{0}\|u\|_{0}^{2}
$$

where $\tilde{\lambda}_{0} \geq 0$.

Proof. We begin by proving that we have with some $\tilde{\lambda}_{0} \geq 0$

$$
\left|B^{q_{1}}(u, u)\right| \geq \operatorname{Re} B^{q_{1}}(u, u) \geq \gamma_{0}\|u\|_{\psi, 1}^{2}-\tilde{\lambda}_{0}\|u\|_{0}^{2} .
$$


For all $u, v \in \mathcal{S}\left(\mathbb{R}^{n}\right)$

$$
\begin{aligned}
\left|B^{q_{1}}(u, u)\right| & \geq \operatorname{Re} B^{q_{1}}(u, u)=\int_{\mathbb{R}^{n}} \operatorname{Re} q_{1}(\xi)|\hat{u}(\xi)|^{2} d \xi \\
& \geq \gamma_{0} \int_{B_{1}^{c}(0)} \psi(\xi)|\hat{u}(\xi)|^{2} d \xi+\int_{B_{1}(0)} \operatorname{Re} q_{1}(\xi)|\hat{u}(\xi)|^{2} d \xi \\
& \geq \gamma_{0}\|u\|_{\psi, 1}^{2}-\gamma_{0}\|u\|_{0}^{2}-\sup _{|\xi| \leq 1}\left(\operatorname{Re} q_{1}(\xi)-\gamma_{0} \psi(\xi) \mid\|u\|_{0}^{2}\right.
\end{aligned}
$$

Therefore, we have proven (2.9). Hence using the above and part of the proof from Theorem 2.4.3, we obtain for $u \in H^{\psi, 1}\left(\mathbb{R}^{n}\right)$

$$
\begin{aligned}
|B(u, u)| & \geq \operatorname{Re} B(u, u) \geq B^{q_{1}}(u, u)-\left|B^{q_{2}}(u, u)\right| \\
& \geq \gamma_{0}\|u\|_{\psi, 1}^{2}-\tilde{\lambda}_{0}\|u\|_{0}^{2}-\kappa_{2} \sum_{|\alpha| \leq n+2}\left\|\varphi_{\alpha}\right\|_{L^{1}}\|u\|_{\psi, 1}^{2} \\
& =\delta_{1}\|u\|_{\psi, 1}^{2}-\tilde{\lambda}\|u\|_{0}^{2}
\end{aligned}
$$

which proves our theorem.

We will now discuss a lengthy example extending some results. For $u, v \in \mathcal{S}\left(\mathbb{R}^{n}\right)$ we consider

$$
B(u, v)=\left(q\left(x, D_{x}\right) u, v\right)_{0}
$$

where we define the operator $q\left(x, D_{x}\right)$ in the following way

$$
q\left(x, D_{x}\right) u(x)=a(y) \psi_{1}\left(D_{z}\right) u(x)+b(z) \psi_{2}\left(D_{y}\right) u(x)
$$

for functions $u: \mathbb{R}^{n} \rightarrow \mathbb{C}$, where $x=(y, z) \in \mathbb{R}^{n_{1}} \times \mathbb{R}^{n_{2}}, n=n_{1}+n_{2}$ and $\psi(\xi)=\psi(\eta, \zeta)=\psi_{1}(\eta)+\psi_{2}(\zeta)$.

Then, for $\mathbb{R}^{n}=\mathbb{R}^{n_{1}} \times \mathbb{R}^{n_{2}}$ and $u(x)=u(y, z)$ the following holds for $u, v \in \mathcal{S}\left(\mathbb{R}^{n}\right)$, where we look at the Fourier transform of $u$ with respect to two variables $y$ and $z$ which we define in the following way.

$$
\begin{aligned}
\left(F_{(x, \xi)} u\right)(x) & =(2 \pi)^{\frac{-n}{2}} \int_{\mathbb{R}^{n}} e^{-i x \xi} u(x) d x \\
& =(2 \pi)^{\frac{-n_{1}}{2}}(2 \pi)^{\frac{-n_{2}}{2}} \int_{\mathbb{R}^{n_{1}}} \int_{\mathbb{R}^{n_{2}}} e^{-i y \eta} e^{-i z \zeta} u(y, z) d z d y \\
& =\left(F_{(y, \eta)}^{(1)} \circ F_{(z, \zeta)}^{(2)} u\right)(x)=\left(F_{(z, \zeta)}^{(2)} \circ F_{(y, \eta)}^{(1)} u\right)(x) .
\end{aligned}
$$


Our aim is to show that

$$
a(y) \psi_{1}\left(D_{z}\right) u(x)=\psi_{1}\left(D_{z}\right)(a u)(x)
$$

and

$$
b(z) \psi_{2}\left(D_{y}\right) u(x)=\psi_{2}\left(D_{y}\right)(b u)(x) .
$$

To prove these equalities we need to show that the commutator described below is equal to 0 , e.g.

$$
\left[a(y), \psi_{1}\left(D_{z}\right)\right] u=0
$$

Theorem 2.4.5. Given an operator $\psi_{1}\left(D_{z}\right)$ on $\mathcal{S}\left(\mathbb{R}^{n}\right)$ defined by

$$
\psi_{1}\left(D_{z}\right) u(x)=(2 \pi)^{\frac{-n_{2}}{2}} \int_{\mathbb{R}^{n_{2}}} e^{-i z \zeta} \psi_{1}(\zeta)(2 \pi)^{\frac{-n_{2}}{2}} \int_{\mathbb{R}^{n_{2}}} e^{i w \zeta} u(y, w) d w d \zeta
$$

where $\psi_{1}: \mathbb{R}^{n_{2}} \rightarrow \mathbb{R}$ is a continuous negative definite function. Then, for the coefficient $a(y)$, we have

$$
\left[a(y), \psi_{1}\left(D_{z}\right)\right]=0 .
$$

for all $y \in \mathbb{R}^{n_{1}}$.

Proof. We find, for two variables $y$ and $z$, where $x=(y, z)$,

$$
\begin{aligned}
a(y) \psi_{1}\left(D_{z}\right) u(y, z) & =a(y)\left(F_{(z, \zeta)}^{(2)}\right)^{-1}\left(\psi_{1}(\zeta) F_{(w, \zeta)}^{(2)} u\right)(y, z) \\
& =a(y)(2 \pi)^{\frac{-n_{2}}{2}} \int_{\mathbb{R}^{n_{2}}} e^{i z \zeta} \psi_{1}(\zeta)\left((2 \pi)^{\frac{-n_{2}}{2}} \int_{\mathbb{R}^{n_{2}}} e^{i w \zeta} u(y, w) d w\right) d \zeta \\
& =(2 \pi)^{\frac{-n_{2}}{2}} \int_{\mathbb{R}^{n_{2}}} e^{i z \zeta} a(y) \psi_{1}(\zeta)\left((2 \pi)^{\frac{-n_{2}}{2}} \int_{\mathbb{R}^{n_{2}}} e^{i w \zeta} u(y, w) d w\right) d \zeta \\
& =(2 \pi)^{\frac{-n_{2}}{2}} \int_{\mathbb{R}^{n_{2}}} e^{i z \zeta} \psi_{1}(\zeta)(2 \pi)^{\frac{-n_{2}}{2}} \int_{\mathbb{R}^{n_{2}}} e^{i w \zeta} a(y) u(y, w) d w d \zeta \\
& =(2 \pi)^{\frac{-n_{2}}{2}} \int_{\mathbb{R}^{n_{2}}} e^{i z \zeta} \psi_{1}(\zeta) F_{(w, \zeta)}^{(2)}(a u)(y, \zeta) d \zeta \\
& =\psi_{1}\left(D_{z}\right)(a u)(y, z) .
\end{aligned}
$$

Hence, we have

$$
\left[a(y), \psi_{1}\left(D_{z}\right)\right] u=a(y) \psi_{2}\left(D_{z}\right) u(y, z)-\psi_{1}\left(D_{z}\right)(a u)(y, z)=0 .
$$


Similarly, we have $\left[b(z), \psi_{2}\left(D_{y}\right)\right] u=0$. We may use these results to look at some estimates regarding the bilinear form $B$. Firstly, we look at the symmetry of the bilinear form.

Theorem 2.4.6. Assume that $|a(y)|,|b(x)| \leq c$. Given the bilinear form

$$
B(u, v)=\left(q\left(x, D_{x}\right) u, v\right)_{0}
$$

Then, the bilinear form is symmetric, i.e. $B(u, v)=B(v, u)$.

Proof. For $u, v \in \mathcal{S}\left(\mathbb{R}^{n}\right)$, due to Theorem 2.4.5 we have the following

$$
\begin{aligned}
\left(q\left(x, D_{x}\right) u, v\right)_{0} & =\left(a \psi_{1}\left(D_{z}\right)+b \psi_{2}\left(D_{y}\right) u, v\right)_{0} \\
& =\left(a \psi_{1}\left(D_{z}\right) u, v\right)_{0}+\left(b \psi_{2}\left(D_{y}\right) u, v\right)_{0} \\
& =\left(u, \psi_{1}\left(D_{z}\right)(a v)\right)_{0}+\left(u, \psi_{2}\left(D_{y}\right)(b v)\right)_{0} \\
& =\left(u, a \psi_{1}\left(D_{z}\right) v\right)_{0}+\left(u, b \psi_{2}\left(D_{y}\right) v\right)_{0} \\
& =\left(u, q\left(x, D_{x}\right) v\right)_{0} .
\end{aligned}
$$

Hence, our statement is proven.

We will now look at some estimates with regard to the bilinear form, where we use the results we obtained in Theorem 2.4.5.

Theorem 2.4.7. Given that $\left[a(y), \psi_{1}\left(D_{z}\right)\right] u=\left[b(z), \psi_{2}\left(D_{y}\right)\right] u=0$, then for every $u, v \in H^{\psi, 1}\left(\mathbb{R}^{n}\right)$, the following estimate holds

$$
|B(u, v)| \leq c\|u\|_{\psi, 1}\|v\|_{\psi, 1}
$$

where $c=\|a\|_{\infty}+\|b\|_{\infty}$. 
Proof. For $u, v \in \mathcal{S}\left(\mathbb{R}^{n}\right)$, we have

$$
\begin{aligned}
|B(u, v)| & \leq\left|\left(a(\cdot) \psi_{1}\left(D_{z}\right) u, v\right)_{0}\right|+\left|\left(b(\cdot) \psi_{2}\left(D_{y}\right) u, v\right)_{0}\right| \\
& =\left|\int_{\mathbb{R}^{n_{1}}} \int_{\mathbb{R}^{n_{2}}} a(y) \psi_{1}\left(D_{z}\right) u(y, z) v(y, z) d z d y\right| \\
& +\left|\int_{\mathbb{R}^{n_{1}}} \int_{\mathbb{R}^{n_{2}}} b(z) \psi_{2}\left(D_{y}\right) u(y, z) v(y, z) d z d y\right| \\
& =\left|\int_{\mathbb{R}^{n_{1}}} \int_{\mathbb{R}^{n_{2}}} a(y) \psi_{1}^{\frac{1}{2}}\left(D_{z}\right) u(y, z) \psi_{1}^{\frac{1}{2}}\left(D_{z}\right) v(y, z) d z d y\right| \\
& +\left|\int_{\mathbb{R}^{n_{1}}} \int_{\mathbb{R}^{n_{2}}} b(z) \psi_{2}^{\frac{1}{2}}\left(D_{y}\right) u(y, z) \psi_{2}^{\frac{1}{2}}\left(D_{y}\right) v(y, z) d z d y\right| \\
& \leq\|a\|_{\infty}\left\|\psi_{1}^{\frac{1}{2}}\left(D_{z}\right) u\right\|_{0}\left\|\psi_{1}^{\frac{1}{2}}\left(D_{z}\right) v\right\|_{0} \\
& +\|b\|_{\infty}\left\|\psi_{2}^{\frac{1}{2}}\left(D_{y}\right) u\right\|_{0}\left\|\psi_{2}^{\frac{1}{2}}\left(D_{y}\right) v\right\|_{0} .
\end{aligned}
$$

We have for $\xi=(\eta, \zeta)$,

$$
\begin{aligned}
\left\|\psi_{1}^{\frac{1}{2}}\left(D_{z}\right) u\right\|_{0}^{2} & =\int_{\mathbb{R}^{n}} \psi_{1}(\zeta)|\hat{u}(\xi)|^{2} d \xi \\
& \leq \int_{\mathbb{R}^{n}} \psi(\xi)|\hat{u}(\xi)|^{2} d \xi \leq\|u\|_{\psi, 1}^{2} .
\end{aligned}
$$

Similarly, we have

$$
\left\|\psi_{2}^{\frac{1}{2}}\left(D_{y}\right) u\right\|_{0}^{2} \leq\|u\|_{\psi, 1}^{2}
$$

Therefore, this implies

$$
\begin{aligned}
|B(u, v)| & \leq\|a\|_{\infty}\|u\|_{\psi, 1}\|v\|_{\psi, 1}+\|b\|_{\infty}\|u\|_{\psi, 1}\|v\|_{\psi, 1} \\
& =\left(\|a\|_{\infty}+\|b\|_{\infty}\right)\|u\|_{\psi, 1}\|v\|_{\psi, 1} .
\end{aligned}
$$

Hence, this proves our estimate for $c=\|a\|_{\infty}+\|b\|_{\infty}$.

Theorem 2.4.8. Given that $\left[a(y) \psi_{1}\left(D_{z}\right)\right] u=\left[b(z), \psi_{2}\left(D_{y}\right)\right] u=0$, then for every $u \in H^{\psi, 1}\left(\mathbb{R}^{n}\right)$, the following estimate holds

$$
B(u, u) \geq \kappa\|u\|_{\psi, 1}^{2}-\kappa\|u\|_{0}^{2}
$$

where we assume that $|a(y)|,|b(z)| \geq \kappa>0$. 
Proof. For $u \in \mathcal{S}\left(\mathbb{R}^{n}\right)$, we have

$$
\begin{aligned}
B(u, u) & =\int_{\mathbb{R}^{n_{1}}} \int_{\mathbb{R}^{n_{2}}} a(y)\left(\psi_{1}\left(D_{z}\right) u(y, z)\right) u(y, z) d z d y \\
& +\int_{\mathbb{R}^{n_{1}}} \int_{\mathbb{R}^{n_{2}}} b(z)\left(\psi_{2}\left(D_{y}\right) u(y, z)\right) u(y, z) d z d y \\
& =\int_{\mathbb{R}^{n_{1}}} \int_{\mathbb{R}^{n_{2}}} a(y)\left(\psi_{1}^{\frac{1}{2}}\left(D_{z}\right) \psi_{1}^{\frac{1}{2}}\left(D_{z}\right) u(y, z)\right) u(y, z) d z d y \\
& +\int_{\mathbb{R}^{n_{1}}} \int_{\mathbb{R}^{n_{2}}} b(z)\left(\psi_{2}^{\frac{1}{2}}\left(D_{y}\right) \psi_{2}^{\frac{1}{2}}\left(D_{y}\right) u(y, z)\right) u(y, z) d z d y \\
& =\int_{\mathbb{R}^{n_{1}}} \int_{\mathbb{R}^{n_{2}}} a(y)\left(\psi_{1}^{\frac{1}{2}}\left(D_{z}\right) u(y, z)\right)^{2} d y d z \\
& +\int_{\mathbb{R}^{n_{1}}} \int_{\mathbb{R}^{n_{2}}} b(z)\left(\psi_{2}^{\frac{1}{2}}\left(D_{y}\right) u(y, z)\right)^{2} d y d z \\
& \geq \kappa\left(\int_{\mathbb{R}^{n_{1}}} \int_{\mathbb{R}^{n_{2}}}\left|\psi_{1}^{\frac{1}{2}}\left(D_{z}\right) u(y, z)\right|^{2} d z d y\right. \\
& \left.+\int_{\mathbb{R}^{n_{1}}} \int_{\mathbb{R}^{n_{2}}}\left|\psi_{2}^{\frac{1}{2}}\left(D_{y}\right) u(y, z)\right|^{2} d y d z\right) \\
& =\kappa\left(\int_{\mathbb{R}^{n_{1}}} \int_{\mathbb{R}^{n_{2}}}\left|\psi_{1}^{\frac{1}{2}}(\zeta)\left(F_{(z, \zeta)} u\right)(y, \zeta)\right|^{2} d \zeta d y\right. \\
& \left.+\int_{\mathbb{R}^{n_{1}}} \int_{\mathbb{R}^{n_{2}}}\left|\psi_{2}^{\frac{1}{2}}(\eta)\left(F_{(y, \eta)} u\right)(\eta, z)\right|^{2} d \eta d z\right) .
\end{aligned}
$$

Calculating the first part, since $\psi_{1}(\zeta) \in \mathbb{R}$ implies $\psi_{1}(\zeta) \geq 0$, we obtain

$$
\begin{aligned}
& \int_{\mathbb{R}^{n_{1}}} \int_{\mathbb{R}^{n_{2}}}\left|\psi_{1}^{\frac{1}{2}}(\zeta)\left(F_{(z, \zeta)} u\right)(y, \zeta)\right|^{2} d \zeta d y \\
& =\int_{\mathbb{R}^{n_{1}}}\left|\psi_{1}^{\frac{1}{2}}(\zeta)\right|^{2} \int_{\mathbb{R}^{n_{2}}}\left|\left(F_{(z, \zeta)} u\right)(y, \zeta)\right|^{2} d y d \zeta \\
& =\int_{\mathbb{R}^{n_{1}}} \int_{\mathbb{R}^{n_{2}}}\left|\psi_{1}^{\frac{1}{2}}(\zeta)\right|^{2}\left|\left(F_{(x, \xi)} u\right)(\eta, \zeta)\right|^{2} d \eta \zeta \\
& =\int_{\mathbb{R}^{n_{1}}} \int_{\mathbb{R}^{n_{2}}}\left|\psi_{1}^{\frac{1}{2}}(\zeta)\right|^{2}|\hat{u}(\eta, \zeta)|^{2} d \eta d \zeta \\
& =\int_{\mathbb{R}^{n_{1}}} \int_{\mathbb{R}^{n_{2}}} \psi_{1}(\zeta)|\hat{u}(\xi)|^{2} d \eta d \zeta .
\end{aligned}
$$

Similary, we have

$$
\begin{aligned}
& \int_{\mathbb{R}^{n_{1}}} \int_{\mathbb{R}^{n_{2}}}\left|\psi_{2}^{\frac{1}{2}}(\eta)\left(F_{(y, \eta)} u\right)(y, z)\right|^{2} d \eta d z \\
& =\int_{\mathbb{R}^{n_{1}}} \int_{\mathbb{R}^{n_{2}}} \psi_{2}(\eta)|\hat{u}(\xi)|^{2} d \eta d \zeta .
\end{aligned}
$$

Hence, we conclude that

$$
\begin{aligned}
B(u, u) & \geq\left(\int_{\mathbb{R}^{n_{1}}} \int_{\mathbb{R}^{n_{2}}}\left(\psi_{1}(\zeta)+\psi_{2}(\eta)\right)|\hat{u}(\xi)|^{2} d \eta d \zeta\right) \\
& =\kappa\|u\|_{\psi, 1}^{2}-\kappa\|u\|_{0}^{2} .
\end{aligned}
$$


We have shown that $B(u, v)$ is bounded, i.e. $|B(u, v)| \leq c\|u\|_{\psi, 1}\|v\|_{\psi, 1}$ for all $u, v \in \mathcal{S}\left(\mathbb{R}^{n}\right)$. Further, we have shown that for some $\kappa$, that the following inequality holds for all $u \in \mathcal{S}\left(\mathbb{R}^{n}\right)$

$$
B(u, u) \geq \kappa\|u\|_{\psi, 1}^{2}-\kappa\|u\|_{0}^{2}
$$

Since the bilinear form on the domain $H^{\psi, 1}$ is bounded from above and below we can conclude that $\left(B, H^{\psi, 1}\right)$ is a closed form on $L^{2}\left(\mathbb{R}^{n}\right)$.

We will now look at some estimates with regard to the operator $q\left(x, D_{x}\right)$.

Theorem 2.4.9. Given that $\left[a(y), \psi_{1}\left(D_{z}\right)\right] u=\left[b(z), \psi_{2}\left(D_{y}\right)\right] u=0$, then for every $u \in H^{\psi, 2}\left(\mathbb{R}^{n}\right)$, the following estimate holds

$$
\left\|q\left(x, D_{x}\right) u\right\|_{\psi, 0} \leq c\|u\|_{\psi, 2}
$$

where $c=\|a\|_{\infty}+\|b\|_{\infty}$

Proof. Here, we follow the structure of the proof for Theorem 2.4.7. For $u \in$ $\mathcal{S}\left(\mathbb{R}^{n}\right)$,

$$
\begin{aligned}
\left\|q\left(x, D_{x}\right) u\right\|_{0} & =\left|\int_{\mathbb{R}^{n_{1}}} \int_{\mathbb{R}^{n_{2}}} a(y) \psi_{1}\left(D_{z}\right) u(y, z) d z d y\right| \\
& +\left|\int_{\mathbb{R}^{n_{1}}} \int_{\mathbb{R}^{n_{2}}} b(z) \psi_{1}\left(D_{y}\right) u(y, z) d z d y\right| \\
& \leq\|a\|_{\infty}\left\|\psi_{1}\left(D_{z}\right) u\right\|_{0} \\
& +\|b\|_{\infty}\left\|\psi_{2}\left(D_{y}\right) u\right\|_{0} .
\end{aligned}
$$

We have for $\xi=(\eta, \zeta)$

$$
\begin{aligned}
\left\|\psi_{1}\left(D_{z}\right) u\right\|_{0}^{2} & =\int_{\mathbb{R}^{n}} \psi_{1}^{2}(\zeta)|\hat{u}(\xi)|^{2} d \xi \\
& \leq \int_{\mathbb{R}^{n}} \psi^{2}(\xi)|\hat{u}(\xi)|^{2} d \xi \\
& \leq \int_{\mathbb{R}^{n}}(1+\psi(\xi))^{2}|\hat{u}(\xi)|^{2} d \xi \\
& =\|u\|_{\psi, 2}^{2} .
\end{aligned}
$$


Hence, we have

$$
\begin{aligned}
\left\|q\left(x, D_{x}\right) u\right\|_{0}^{2} & \leq\|a\|_{\infty}\|u\|_{\psi, 2}^{2}+\|b\|_{\infty}\|u\|_{\psi, 2}^{2} \\
& =\left(\|a\|_{\infty}+\|b\|_{\infty}\right)\|u\|_{\psi, 2}^{2} .
\end{aligned}
$$

This proves our estimate for $c=\|a\|_{\infty}+\|b\|_{\infty}$.

We may now look at the case for the lower bound.

Theorem 2.4.10. Given that $\left[a(y), \psi_{1}\left(D_{z}\right)\right] u=\left[b(z), \psi_{2}\left(D_{y}\right)\right] u=0$, then for every $u \in H^{\psi, 2}\left(\mathbb{R}^{n}\right)$, the following estimate holds

$$
\left\|q\left(x, D_{x}\right) u\right\|_{\psi, 0} \geq \kappa(1-2 \gamma)\|u\|_{\psi, 2}-\lambda_{0}\|u\|_{0}
$$

for $\gamma<1$, where we assume that $\sup _{y \in \mathbb{R}^{n_{1}}}\left|a(y)-a\left(y_{0}\right)\right|<\gamma \kappa$ and $\sup _{y \in \mathbb{R}^{n_{2}}} \mid b(y)$ $b\left(y_{0}\right) \mid<\gamma \kappa$.

Proof. Firstly, we rewrite the operator as follows

$$
q\left(x, D_{x}\right)=q\left(x_{0}, D_{x}\right)-\left(q\left(x_{0}, D_{x}\right)-q\left(x, D_{x}\right)\right)
$$

where we "freeze" the coefficients at $x_{0}$. Therefore, we have

$$
\left\|q\left(x, D_{x}\right) u\right\|_{0} \geq\left\|q\left(x_{0}, D_{x}\right) u\right\|_{0}-\left\|\left(q\left(x_{0}, D_{x}\right)-q\left(x, D_{x}\right)\right) u\right\|_{0}
$$

Following the calculations as in Theorem 2.4.8, we obtain

$$
\left\|q\left(x_{0}, D_{x}\right) u\right\|_{0} \geq \kappa\|u\|_{\psi, 2}-\lambda_{0}\|u\|_{0}
$$

Thus, we have remaining the norm $\left\|\left(q\left(x_{0}, D_{x}\right)-q\left(x, D_{x}\right)\right) u\right\|_{0}$ to estimate. For $u \in \mathcal{S}\left(\mathbb{R}^{n}\right)$, we have

$$
\begin{aligned}
\left\|\left(q\left(x_{0}, D_{x}\right)-q\left(x, D_{x}\right)\right) u\right\|_{0}^{2} & =\int_{\mathbb{R}^{n_{1}}} \int_{\mathbb{R}^{n_{2}}} \mid a(y) \psi_{1}\left(D_{z}\right) u(y, z)+b(z) \psi_{2}\left(D_{y}\right) u(y, z) \\
& -a\left(y_{0}\right) \psi_{1}\left(D_{z}\right) u(y, z)-\left.b\left(z_{0}\right) \psi_{2}\left(D_{y}\right) u(y, z)\right|^{2} d z d y \\
& =\int_{\mathbb{R}^{n_{1}}} \int_{\mathbb{R}^{n_{2}}} \mid\left(a(y)-a\left(y_{0}\right)\right) \psi_{1}\left(D_{z}\right) u(y, z) \\
& +\left.\left(b(z)-b\left(z_{0}\right)\right) \psi_{2}\left(D_{y}\right) u(y, z)\right|^{2} d z d y \\
& \leq\left. 2 \int_{\mathbb{R}^{n_{1}}} \int_{\mathbb{R}^{n_{2}}}\left(\mid a(y)-a\left(y_{0}\right) \psi_{1}\left(D_{z}\right)\right) u(y, z)\right|^{2} \\
& \left.+\left|b(y)-b\left(y_{0}\right) \psi_{2}\left(D_{y}\right) u(y, z)\right|^{2}\right) d z d y .
\end{aligned}
$$


Due to the fact that we have the estimates

$$
\sup _{y \in \mathbb{R}^{n_{1}}}\left|a(y)-a\left(y_{0}\right)\right|<\gamma \kappa
$$

and

$$
\sup _{y \in \mathbb{R}^{n_{2}}}\left|b(y)-b\left(y_{0}\right)\right|<\gamma \kappa,
$$

from the calculation obtained in the previous theorem, we conclude that

$$
\begin{aligned}
\left\|\left(q\left(x_{0}, D_{x}\right)-q\left(x, D_{x}\right)\right) u\right\|_{0}^{2} & \leq 2 \gamma^{2} \kappa^{2} \int_{\mathbb{R}^{n_{1}}} \int_{\mathbb{R}^{n_{2}}}\left|\psi_{1}\left(D_{z}\right) u(y, z)\right|^{2}+\left|\psi_{2}\left(D_{y}\right) u(y, z)\right|^{2} d z d y \\
& \leq 2 \gamma^{2} \kappa^{2}\|u\|_{\psi, 2}^{2} .
\end{aligned}
$$

Hence, we have

$$
\left\|q\left(x, D_{x}\right) u\right\|_{0} \geq \kappa\|u\|_{\psi, 2}-\lambda_{0}\|u\|_{0}-2 \gamma \kappa\|u\|_{\psi, 2}=\kappa(1-2 \gamma)\|u\|_{\psi, 2}-\lambda_{0}\|u\|_{0}
$$

where $\gamma<1$.

We want to show that as a result of these estimates we have that this operator has an extension generating a $L^{2}$ sub-Markovian semigroup. In order to do this, since we have shown in Theorem 2.3.8 that there is a one-to-one correspondence between Dirichlet forms and $L^{2}$ sub-Markovian semigroups, we need to show that $\left(B, H^{\psi, 1}\right)$ is a Dirichlet form. Firstly, we will show for the general case that the following equality holds.

$$
\int_{\mathbb{R}^{n}} \psi(\xi) \hat{u}(\xi) \overline{\hat{v}(\xi)} d \xi=c \int_{\mathbb{R}^{n}} \int_{\mathbb{R}^{n}}(u(x+y)-u(x))(v(x+y)-v(x)) \nu(d y) d x .
$$

For the symmetric case, $\psi$ has a Lévy-Khinchin representation

$$
\psi(\xi)=\int_{\mathbb{R}^{n}}(1-\cos (y \cdot \xi)) \tilde{\nu}(d y)
$$

where we have no diffusion along with no killing term regarding the quadratic form and constant.

Note, that for $f, g \in \mathcal{S}\left(\mathbb{R}^{n}\right)$,

$$
\int_{\mathbb{R}^{n}} f(t) g(-t) d t=(2 \pi)^{-n} \int_{\mathbb{R}^{n}} \int_{\mathbb{R}^{n}} \int_{\mathbb{R}^{n}} e^{i(x+y) z} f(x) g(y) d x d y d z .
$$


Hence, we have

$$
\begin{aligned}
& \int_{\mathbb{R}^{n}} \int_{\mathbb{R}^{n}}(1-\cos (y \cdot \xi)) F u(\xi) \overline{F v(\xi)} \tilde{\nu}(d y) d \xi \\
& =\frac{1}{2} \int_{\mathbb{R}^{n}} \int_{\mathbb{R}^{n}}\left(e^{i y \cdot \xi}-1\right)\left(e^{-i y \cdot \xi}-1\right) F u(\xi) F v(-\xi) d \xi \tilde{\nu}(d y) \\
& =\frac{(2 \pi)^{-n}}{2} \int_{\mathbb{R}^{n}} \int_{\mathbb{R}^{n}} \int_{\mathbb{R}^{n}} \int_{\mathbb{R}^{n}} e^{i \sigma \cdot x}\left(e^{i y \cdot \sigma}-1\right) F u(\sigma) \\
& \times e^{i \tau \cdot x}\left(e^{i y \cdot \tau}-1\right) F v(\tau) d \sigma d \tau \tilde{\nu}(d y) d x \\
& =\frac{1}{2} \int_{\mathbb{R}^{n}} \int_{\mathbb{R}^{n}}(u(x+y)-u(x))(v(x+y)-v(x)) \tilde{\nu}(d y) d x .
\end{aligned}
$$

We may use this to rewrite the bilinear form in the following way.

For $u, v \in H^{\psi, 1}\left(\mathbb{R}^{n}\right)$,

$$
\begin{aligned}
B(u, v) & =\int_{\mathbb{R}^{n}} \int_{\mathbb{R}^{n}} a(y) \psi_{1}\left(D_{z}\right) u(y, z) v(y, z) d z d y \\
& +\int_{\mathbb{R}^{n}} \int_{\mathbb{R}^{n}} b(z) \psi_{2}\left(D_{y}\right) u(y, z) v(y, z) d z d y \\
& =\frac{1}{2} \int_{\mathbb{R}^{n}} \int_{\mathbb{R}^{n}} a(y)(u(y, z+\tilde{z})-u(y, z))(v(y, z+\tilde{z})-v(y, z)) \nu_{2}(d \tilde{z}) d z d y \\
& +\frac{1}{2} \int_{\mathbb{R}^{n}} \int_{\mathbb{R}^{n}} b(z)(u(y+\tilde{y}, z)-u(y, z))(v(y+\tilde{y}, z)-v(y, z)) \nu_{1}(d \tilde{y}) d y d z
\end{aligned}
$$

Note, for all $u, v \in L^{2}\left(\mathbb{R}^{n}\right)$, we have

$$
((u \vee 0) \wedge 1)(x+y)-((u \vee 0) \wedge 1)(x))^{2} \leq(u(x+y)-u(x))^{2}
$$

This implies that $\left(B, H^{\psi, 1}\right)$ is a symmetric Dirichlet form. We can state this formally as a theorem.

Theorem 2.4.11. Given the bilinear form B defined by (2.10) on the domain $H^{\psi, 1}$ where (2.14) and (2.15) holds, then $\left(B, H^{\psi, 1}\right)$ is a symmetric Dirichlet form.

Due to the fact that within the proof of Theorem 2.4.6 we have shown that

$$
\left(q\left(x, D_{x}\right) u, v\right)=\left(u, q\left(x, D_{x}\right) v\right)
$$

which holds for all $u, v \in H^{\psi, 2}$, we can say that the operator $q\left(x, D_{x}\right)$ is symmetric. Therefore, by Friedrichs' extension theorem, Theorem 2.1.18, the operator 
has a self-adjoint extension. In particular, from our estimates, along with the analysis obtained in the beginning of the chapter, we find that the operator $q\left(x, D_{x}\right)$ has a self-adjoint extension generating a $L^{2}$ sub-Markovian semigroup on the domain $H^{\psi, 2}$. Moreover we have

Theorem 2.4.12. Given that $\left[a(y), \psi_{1}\left(D_{z}\right)\right] u=\left[b(z), \psi_{2}\left(D_{y}\right)\right] u=0$ then the estimates from Theorems 2.4.7-2.4.10 hold. Suppose that in addition Theorem 2.4.11 holds. Then we can say that the operator, $q\left(x, D_{x}\right)$, has a self-adjoint extension generating a strongly continuous symmetric sub-Markovian semigroup on $L^{2}\left(\mathbb{R}^{n}\right)$.

Proof. Firstly, given an operator defined by

$$
q\left(x, D_{x}\right) u(x)=a(y) \psi_{1}\left(D_{z}\right) u(x)+b(z) \psi_{2}\left(D_{y}\right) u(x),
$$

where we have shown within the proof of Theorem 2.4.6 that

$$
\left(q\left(x, D_{x}\right) u, v\right)=\left(u, q\left(x, D_{x}\right) v\right)
$$

holds for all $u, v \in H^{\psi, 2}$. Therefore, we can say that the operator $q\left(x, D_{x}\right)$ is symmetric. As a result, due to Friedrichs' extension theorem, the operator has a self-adjoint extension. From our estimates earlier in the chapter, we can conclude that $\left(q\left(x, D_{x}\right), H^{\psi, 2}\left(\mathbb{R}^{n}\right)\right)$ is a densely defined closed operator on $L^{2}\left(\mathbb{R}^{n}\right)$. Since we have stated that the operator has a self-adjoint extension, we can conclude that the operator, $q\left(x, D_{x}\right)$, has a self-adjoint extension which generates a strongly continuous symmetric sub-Markovian semigroup on $L^{2}\left(\mathbb{R}^{n}\right)$.

Note that this example extends the results in [21] since we do not need any differentiability assumption on the coefficients. 


\section{Chapter 3}

\section{Metric Measure Spaces}

\subsection{Transition Densities and Metric Measure Space}

We want to study transition densities of Lévy and Lévy-type processes and we aim to relate them to some geometry induced by the symbol of their generator. In order to do this we need to study metric measure spaces whose metric is induced by a continuous negative definite function. Let $\psi: \mathbb{R}^{n} \rightarrow \mathbb{R}$ be a locally bounded continuous negative definite function. Note that real-valued continuous negative definite functions are non-negative,

$$
0 \leq \psi(\xi) \leq c_{\psi}\left(1+|\xi|^{2}\right) \text { with } c_{\psi}+2 \sup _{|\eta| \leq 1} \psi(\eta)
$$

and subadditive, i.e.

$$
\sqrt{\psi(\xi+\eta)} \leq \sqrt{\psi(\xi)}+\sqrt{\psi(\eta)}
$$

The defining property of continuous negative definite functions imply that whenever $\psi\left(\xi_{0}\right)=0$ for some $\xi_{0} \neq 0$ then $\psi$ is periodic with period $\xi_{0}$. In fact, for a continuous negative definite function $\psi$ to be non-periodic (and non-constant) is equivalent to $\psi(\xi)=0$ implies $\xi=0$. Since $\psi(-\xi)=\overline{\psi(\xi)}$ and by the subadditive property we can see that every locally bounded, non-periodic negative 
definite function with $\psi(0)=0$ induces a metric on $\mathbb{R}^{n}$ by

$$
d_{\psi}: \mathbb{R}^{n} \times \mathbb{R}^{n} \rightarrow[0, \infty), d_{\psi}(\xi, \eta):=\sqrt{\psi(\xi-\eta)} .
$$

Thus, as we have shown in Lemma 1.2.10 if we require that $\psi(\xi)=0$ if, and only if, $\xi=0$, then $d_{\psi}(\xi, \eta):=\sqrt{\psi(\xi-\eta)}$ generates a metric on $\mathbb{R}^{n}$.

Let $\psi \in \mathcal{M C N}$, where we denote by $\mathcal{M C N}$ the continuous negative definite functions that induce a metric on $\mathbb{R}^{n}$ which generates the Euclidean topology. We are interested in the metric measure space $\left(\mathbb{R}^{n}, d_{\psi}, \lambda^{(n)}\right)$ and the notion of volume doubling plays a vital role in the analysis on metric measure spaces. A metric measure space is a triple $(X, d, \mu)$ where $(X, d)$ is a metric space and $\mu$ is a measure on the Borel sets of the space $X$. The following definition is taken from $[23]$.

Definition 3.1.1. Let $(X, d, \mu)$ be a metric measure space. We say that $(X, d, \mu)$ or $\mu$ has the volume doubling property if there exists a constant $c_{2}$ such that

$$
\mu\left(B^{d}(x, 2 r)\right) \leq c_{2} \mu\left(B^{d}(x, r)\right)
$$

holds for all metric balls $B^{d}(x, r)=\{y \in X: d(y, x)<r\} \subset X$.

The volume doubling property will be useful in proving Theorem 3.1.2. Let $\psi \in \mathcal{M C N}$ and denote by $\left(\mu_{t}\right)_{t \geq 0}$ the corresponding convolution semigroup with $F^{-1} \mu_{t}=e^{-t \psi}$. We assume that $e^{-t \psi} \in L^{1}\left(\mathbb{R}^{n}, \lambda^{(n)}\right)$, so that the measures $\mu_{t}$ are absolutely continuous with respect to the Lebesgue measure. The (transition) density is given by

$$
p_{t}(x)=(2 \pi)^{-n} \int_{\mathbb{R}^{n}} e^{-i x \xi} e^{-t \psi(\xi)} d \xi=(2 \pi)^{-\frac{n}{2}} F e^{-t \psi}(x), \quad t>0,
$$

i.e. $\mu_{t}=p_{t}(\cdot) \lambda^{(n)}$. We will discuss the relation of the transition density at $0, p_{t}(0)$, in relation to the geometry induced by the metric measure space $\left(\mathbb{R}^{n}, d_{\psi}, \lambda^{(n)}\right)$. The proof of the following theorem is taken from [23], a similar result can essentially be found in [27].

Theorem 3.1.2. Let $\psi \in \mathcal{M C N}\left(\mathbb{R}^{n}\right)$ and assume that $e^{-t \psi} \in L^{1}\left(\mathbb{R}^{n}, \lambda^{(n)}\right)$. Then

$$
p_{t}(0)=(2 \pi)^{-n} \int_{0}^{\infty} \lambda^{(n)}\left(B^{d_{\psi}}\left(0, \sqrt{\frac{r}{t}}\right)\right) e^{-r} d r, t>0
$$


If the metric measure space $\left(\mathbb{R}^{n}, d_{\psi}, \lambda^{(n)}\right)$ has the volume doubling property, then $e^{-t \psi} \in L^{1}\left(\mathbb{R}^{n}, \lambda^{(n)}\right)$ and

$$
p_{t}(0) \asymp \lambda^{(n)}\left(B^{d_{\psi}}\left(0, \frac{1}{\sqrt{t}}\right)\right) \text { for all } t>0 .
$$

Proof. Observe that $\lambda^{(n)}\left(B^{d_{\psi}}(0, \sqrt{\rho})\right)=\lambda^{(n)}\left\{\xi \in \mathbb{R}^{n}: \psi(\xi)<\rho\right\}$ is the distribution function of $\psi$. Using Fubini's theorem and the change of variable $\rho=\frac{r}{t}$ we get

$$
\begin{aligned}
(2 \pi)^{n} p_{t}(0) & =\int_{\mathbb{R}^{n}} e^{-t \psi(\xi)} d \xi=-t \int_{0}^{\infty} \lambda^{(n)}\left\{\xi \in \mathbb{R}^{n}: \psi(\xi) \geq \rho\right\} e^{-t \rho} d \rho \\
& =t \int_{0}^{\infty} \lambda^{(n)}\left\{\xi \in \mathbb{R}^{n}: \psi(\xi)<\rho\right\} e^{-t \rho} d \rho=t \int_{0}^{\infty} \lambda^{(n)}\left(B^{d_{\psi}}(0, \sqrt{\rho})\right) e^{-t \rho} d \rho \\
& =\int_{0}^{\infty} \lambda^{(n)}\left(B^{d_{\psi}}\left(0, \sqrt{\frac{r}{t}}\right)\right) e^{-r} d r
\end{aligned}
$$

and (3.1) follows.

Using (3.1) and the monotonicity of the function $r \mapsto \lambda^{(n)}\left(B^{d_{\psi}}(0, r)\right)$ we get

$$
\begin{aligned}
(2 \pi)^{n} p_{t}(0) & \geq \int_{1}^{\infty} \lambda^{(n)}\left(B^{d_{\psi}}\left(0, \sqrt{\frac{r}{t}}\right)\right) e^{-r} d r \geq \lambda^{(n)}\left(B^{d_{\psi}}\left(0, \frac{1}{\sqrt{t}}\right)\right) \int_{1}^{\infty} e^{-r} d r \\
& =\frac{1}{e} \lambda^{(n)}\left(B^{d_{\psi}}\left(0, \frac{1}{\sqrt{t}}\right)\right) .
\end{aligned}
$$

This proves the first inequality of (3.2). The upper estimate requires that $d_{\psi}$ enjoys the volume doubling property stated in Definition 3.1.1 which gives

$$
\lambda^{(n)}\left(B^{d_{\psi}}(0, c r)\right) \leq \gamma_{0}(c) \lambda^{(n)}\left(B^{d_{\psi}}(0, r)\right), c>1, r>0,
$$

for some function $\gamma_{0}$ such that $\gamma_{0}(c) \leq \gamma_{0}(1) c^{\alpha}$ for all $c \geq 1$ with some suitable constant $\alpha \geq 0$. Combining this with (3.1) gives

$$
\begin{aligned}
(2 \pi)^{n} p_{t}(0) & =\int_{0}^{1} \lambda^{(n)}\left(B^{d_{\psi}}\left(0, \sqrt{\frac{r}{t}}\right)\right) e^{-r} d r+\int_{1}^{\infty} \lambda^{(n)}\left(B^{d_{\psi}}\left(0, \sqrt{\frac{r}{t}}\right)\right) e^{-r} d r \\
& \leq\left(1-e^{-1}\right) \lambda^{(n)}\left(B^{d_{\psi}}\left(0, \frac{1}{\sqrt{t}}\right)\right)+\lambda^{(n)}\left(B^{d_{\psi}}\left(0, \frac{1}{\sqrt{t}}\right)\right) \int_{1}^{\infty} \gamma_{0}(1) r^{\frac{\alpha}{2}} e^{-r} d r \\
& =\kappa_{1} \lambda^{(n)}\left(B^{d_{\psi}}\left(0, \frac{1}{\sqrt{t}}\right)\right)
\end{aligned}
$$

this is the upper estimate in (3.2). The statement that the volume doubling property implies $e^{-t \psi} \in L^{1}\left(\mathbb{R}^{n} ; \lambda^{(n)}\right)$ is proved in [27]. 


\subsection{Examples Involving some Transition Den- sities}

We will now give some examples which illustrate Theorem 3.1.2, where we take part of the calculation from [29].

Example 3.2.1. Let $f(s)=s^{\alpha}, 0<\alpha<1$ be a Bernstein function. Since we know that $\psi:=f\left(|\cdot|^{2}\right)$ is in $\mathcal{M C N}\left(\mathbb{R}^{n}\right)$ it follows that

$$
\lambda\left(B^{d_{\left.|\cdot|\right|^{2 \alpha}}}(0, c r)\right)=c^{\frac{n}{\alpha}} \lambda\left(B^{d_{|\cdot| 2 \alpha}}(0, r)\right) .
$$

Since $\lambda\left(B^{d_{\left.1 \cdot\right|^{2 \alpha}}}(0, r)\right)=c_{n, \alpha} r^{\frac{n}{\alpha}}$ we obtain the estimate

$$
p_{t}^{(2 \alpha)}(0) \asymp t^{-\frac{n}{2 \alpha}},
$$

where $p_{t}^{(2 \alpha)}(x)$ is the transition density of the symmetric $2 \alpha$-stable Lévy process.

Example 3.2.2. Consider on $\mathbb{R}^{n_{1}} \times \mathbb{R}^{n_{2}}$ the function $\psi(\xi, \eta)=|\xi|^{\alpha}+|\eta|^{\beta}$ with $0<\alpha<\beta<2$. Then $\psi \in \mathcal{M C N}\left(\mathbb{R}^{n_{1}} \times \mathbb{R}^{n_{2}}\right)$. We have

$$
\begin{aligned}
B^{\psi}(0, \rho) & =\left\{(x, y) \in \mathbb{R}^{n_{1}} \times \mathbb{R}^{n_{2}}:\left(|x|^{\alpha}+|y|^{\beta}\right)^{\frac{1}{2}}<\rho\right\} \\
& =\left\{(x, y) \in \mathbb{R}^{n_{1}} \times \mathbb{R}^{n_{2}}:\left|\frac{x}{\rho^{\frac{2}{\alpha}}}\right|^{\alpha}+\left|\frac{y}{\rho^{\frac{2}{\beta}}}\right|^{\beta}<1\right\} .
\end{aligned}
$$

The Lebegue measure of $B^{\psi}(0, \rho)$ is given by:

$$
\begin{aligned}
\lambda^{(n)}\left(B^{\psi}(0, \rho)\right) & =\int_{\mathbb{R}^{n_{1}}} \int_{\mathbb{R}^{n_{2}}} \chi_{B_{1}^{\psi}}\left(\frac{x}{\rho^{\frac{2}{\alpha}}}, \frac{y}{\rho^{\frac{2}{\beta}}}\right) d y d x \\
& =\rho^{\frac{2 n_{1}}{\alpha}} \rho^{\frac{2 n_{2}}{\beta}} \int_{\mathbb{R}^{n_{1}}} \int_{\mathbb{R}^{n_{2}}} \chi_{B_{1}^{\psi}}(\xi, \eta) d \eta d \xi=\rho^{2\left(\frac{n_{1}}{\alpha}+\frac{n_{2}}{\beta}\right)} \lambda^{(n)}\left(B^{\psi}(0,1)\right)
\end{aligned}
$$

where we used the change of variables $\xi:=\frac{x}{\rho^{\frac{2}{\alpha}}}, \eta:=\frac{y}{\rho^{\frac{2}{\beta}}}$, therefore $d x=\rho^{\frac{2 n_{1}}{\alpha}} d \xi$ and $d y=\rho^{\frac{2 n_{2}}{\beta}} d \eta$.

Consequently, we get for $p_{t}^{\psi}(0)=(2 \pi)^{-n_{1}-n_{2}} \iint_{\mathbb{R}^{n_{1}} \times \mathbb{R}^{n_{2}}} e^{-t\left(|\xi|^{\alpha}+|\eta|^{\beta}\right)} d \xi d \eta$ that

$$
p_{t}^{\psi}(0) \asymp t^{-\frac{n_{1}}{\alpha}-\frac{n_{2}}{\beta}} .
$$


We have seen above that $p_{t}(0)$ has a natural meaning in the metric measure space $\left(\mathbb{R}^{n}, d_{\psi}, \lambda\right)$. Moreover, we have

$$
\frac{p_{t}(x)}{p_{t}(0)}=\int_{\mathbb{R}^{n}} e^{-i x \xi} \frac{e^{-d_{\psi}^{2}(\xi, 0)}}{(2 \pi)^{n} p_{t}(0)} d \xi=\int_{\mathbb{R}^{n}} e^{-i x \xi} \frac{e^{-t \psi(\xi)}}{(2 \pi)^{n} p_{t}(0)} d \xi,
$$

where we want to understand $\frac{p_{t}(x)}{p_{t}(0)}$. We have some examples to illustrate this.

Example 3.2.3. Let $\psi(\xi)=\frac{1}{2}|\xi|^{2}$. Then $p_{t}(x)$ is the Gauss kernel in $\mathbb{R}^{n}$,

$$
\frac{p_{t}(x)}{p_{t}(0)}=\exp \left(-\frac{|x|^{2}}{2 t}\right) \text {. }
$$

and for the metric $\phi_{t}(x, y)=\frac{|x-y|}{\sqrt{2 t}}$ we obtain

$$
\frac{p_{t}(x)}{p_{t}(0)}=\exp \left(-\phi_{t}^{2}(x, 0)\right)
$$

Example 3.2.4. Let $\psi(\xi)=|\xi|$. Then $p_{t}(x)$ is the density of the Cauchy process in $\mathbb{R}^{n}$ and we find

$$
\frac{p_{t}(x)}{p_{t}(0)}=\exp \left(-\phi_{t}^{2}(x, 0)\right)
$$

with

$$
\phi_{t}(x, y)=\sqrt{\frac{n+1}{2} \ln \left(\frac{|x-y|^{2}+t^{2}}{t^{2}}\right)} .
$$

We will now give an interpretation of (3.3). We have

$$
p_{t}(x)=p_{t}(0) \exp \left(-\delta_{\psi}^{2}(t, x, 0)\right)
$$

where the map $\delta_{\psi}(t, \cdot, \cdot): \mathbb{R}^{n} \times \mathbb{R}^{n} \rightarrow \mathbb{R}$ is a metric and if $\psi$ satisfies the volume doubling condition, we get from Theorem 3.1.2.

$$
p_{t}(x) \asymp \lambda\left(B^{d_{\psi}}\left(0, \frac{1}{\sqrt{t}}\right)\right) \exp \left(-\delta_{\psi}^{2}(t, x, 0)\right)
$$

with the balls $B^{d_{\psi}}(0, r)=\left\{y \in \mathbb{R}^{n}: d_{\psi}(y, 0)<r\right\}$.

Example 3.2.5. The symmetric Meixner process on $\mathbb{R}$ has the Lévy exponent, i.e. the symbol, $\psi(\xi)=\ln (\cosh \xi)$ and the transition density

$$
p_{t}(x)=\frac{2^{t-1}}{\pi \Gamma(t)}\left|\Gamma\left(\frac{t+i x}{2}\right)\right|^{2} .
$$


Using the representation of the Gamma function as an infinite product, we find

$$
\frac{p_{t}(x)}{p_{t}(0)}=\left|\frac{\Gamma\left(\frac{t+i x}{2}\right)}{\Gamma\left(\frac{t}{2}\right)}\right|^{2}=\prod_{j=1}^{\infty}\left(1+\frac{x^{2}}{(t+2 j)^{2}}\right)^{-1} .
$$

Since for a sequence $\left(a_{j}\right)_{j \geq 1}$ of positive numbers the convergence of $\prod_{j=1}^{\infty}\left(1+a_{j}\right), \sum_{j=1}^{\infty} \ln \left(1+a_{j}\right)$ and $\sum_{j=1}^{\infty} a_{j}$ is equivalent, we find that

$$
\delta_{t}^{2}(x, 0)=-\ln \left|\frac{\Gamma\left(\frac{t+i x}{2}\right)}{\Gamma\left(\frac{t}{2}\right)}\right|^{2}=\sum_{j=1}^{\infty} \ln \left(1+\frac{x^{2}}{(t+2 j)^{2}}\right) .
$$

For every $j \geq 1$ and $t>0$ the function $x \mapsto \ln \left(1+x^{2}(t+2 j)^{-2}\right)$ is continuous and negative definite. Since the series $\sum_{j=1}^{\infty} \ln \left(1+x^{2}(t+2 j)^{-2}\right)$ converges locally uniformly as a function of $x$, its sum $\delta_{t}^{2}(x, 0)$ is again a continuous negative definite function. This shows that the transition density of a symmetric Meixner process satisifies

$$
p_{t}(x)=p_{t}(0) e^{\left(-\delta_{t}^{2}(x, 0)\right)}
$$

We can also write $p_{t}(0)$ as

$$
p_{t}(0)=(2 \pi)^{-n} \int_{\mathbb{R}^{n}} e^{-t \ln (\cosh \xi)} d \xi
$$

Thus if $\left(\mathbb{R}^{n}, d_{\psi}, \lambda\right)$ has the volume doubling property, $p_{t}(x)$ is controlled by two geometric expressions. Therefore when discussing examples of the corresponding density of certain continuous negative definite functions which we relate to certain metrics in chapter 6 we may consider whether we have control with regard to two geometric expressions as we do above. 


\section{Chapter 4}

\section{Distributions in the Sense of L.Schwartz and Fundamental Solutions}

\subsection{Distributions}

We introduce the notion of a distribution which is taken from [10].

Definition 4.1.1. Let $G \subset \mathbb{R}^{n}$ be an open set. A linear form $u: C_{0}^{\infty}(G) \rightarrow \mathbb{C}$ is called a distribution if, for every compact set $K \subset G$, there is a real number $C \geq 0$ and a non negative integer $N$ such that

$$
|\langle u, \phi\rangle| \leq C \sum_{|\alpha| \leq N} \sup \left|\partial^{\alpha} \phi\right|
$$

for all $\phi \in C_{0}^{\infty}(G)$ with supp $\phi \subset K$. The vector space of distributions on $G$ is called $\mathscr{D}^{\prime}(G)$.

We will now give a simple example to illustrate this.

Theorem 4.1.2. Let $G \subset \mathbb{R}^{n}$ be an open set, and let $g \in C^{0}(G)$. Then

$$
\langle g, \phi\rangle=\int g \phi d x, \phi \in C_{0}^{\infty}(G)
$$


is a distribution. Moreover, for a locally finite measure $\mu$ on $\mathcal{B}(G)$ we may define

$$
\langle\mu, \phi\rangle=\int \phi \mu(d x)
$$

which also gives a distribution.

This includes the Dirac distribution which belongs to $\mathscr{D}^{\prime}\left(\mathbb{R}^{n}\right)$ defined by

$$
\left\langle\varepsilon_{0}, \phi\right\rangle=\phi(0), \phi \in C_{0}^{\infty}\left(\mathbb{R}^{n}\right)
$$

There is an equivalent way of defining distributions.

Theorem 4.1.3. A linear form $u$ on $C_{0}^{\infty}(G)$ is a distribution if and only if $\lim _{j \rightarrow \infty}\left\langle u, \phi_{j}\right\rangle=0$ for every sequence $\phi_{j}$ that has the following properties.

(i) The supports of all the $\phi_{j}$ are in a fixed compact set,

(ii) $\partial^{\alpha} \phi_{j} \rightarrow 0$, uniformly as $j \rightarrow \infty$, for every multi-index $\alpha$.

We can state Definition 4.1.1 differently:

Theorem 4.1.4. Let $G \subset \mathbb{R}^{n}$ be an open set. The topological dual space $\mathscr{D}^{\prime}(G)$ of $C_{0}^{\infty}(G)$ is the space of distributions on $G$.

A basic operation of distributions going beyond the vector space operations is that of differentiation.

Let $G \subset \mathbb{R}^{n}$ be an open set. If $u \in C^{1}(G)$, then the distribution which is equal to the derivative $\partial_{i} u$, where $i=1, \ldots, n$, is

$$
\left\langle\partial_{i} u, \phi\right\rangle=\int \phi \partial_{i} u d x=-\int u \partial_{i} \phi d x=-\left\langle u, \partial_{i} \phi\right\rangle, \quad \phi \in C_{0}^{\infty}(G)
$$

where we have used integration by parts.

Definition 4.1.5. Let $u \in \mathscr{D}^{\prime}(G)$. The distributions $\partial_{i} u, i=1, \ldots, n$, given by (4.1), are called the first order derivatives of $u$. 
We can iterate (4.1) to obtain for $u \in \mathscr{D}^{\prime}(G)$

$$
\left\langle\partial^{\alpha} u, \phi\right\rangle=(-1)^{|\alpha|}\left\langle u, \partial^{\alpha} \phi\right\rangle, \phi \in C_{0}^{\infty}(G),
$$

and therefore a distribution has derivatives of all orders. An example is that of the Heaviside function.

Example 4.1.6. Given the Heaviside function defined by

$$
H(x)=1, x>0 ; H(x)=0, x \leq 0 .
$$

Its distributional derivative is given by

$$
\langle\partial H, \phi\rangle=-\langle H, \partial \phi\rangle=-\int_{0}^{\infty} \partial \phi(x) d x=\phi(0), \phi \in C_{0}^{\infty}(\mathbb{R}) .
$$

Hence $\partial H=\varepsilon_{0}$ in the sense of distributions. For a fixed $a \in \mathbb{R}$ a similar manipulation gives

$$
\partial H(\cdot-a)=\varepsilon_{a} .
$$

By (4.2) the higher order derivatives of the Dirac distribution are

$$
\left\langle\partial^{k} \varepsilon_{a}, \phi\right\rangle=(-1)^{k} \partial^{k} \phi(a), \quad k=1, \ldots, \phi \in C_{0}^{\infty}(\mathbb{R}) .
$$

We will now look at a subspace of the vector space of distributions. In particular, the distributions with compact support which is taken from [10].

Definition 4.1.7. Let $G \subset \mathbb{R}^{n}$ be an open set. A linear form $u$ on the vector space $C^{\infty}(G)$ is called continuous if there are a compact set $K \subset G$, a constant $C \geq 0$ and a non negative integer $N$ such that, for all $\phi \in C^{\infty}(G)$

$$
|\langle u, \phi\rangle| \leq C \sum_{|\alpha| \leq N} \sup \left\{\left|\partial^{\alpha} \phi\right|: x \in K\right\} .
$$

The vector space of continuous linear forms on $C^{\infty}(G)$ is called $\mathcal{E}^{\prime}(G)$.

We know that $C_{0}^{\infty}(G) \subset C^{\infty}(G)$. Therefore it follows from Definition 4.1.7 that the restriction of any $u \in \mathcal{E}^{\prime}(G)$ to $C_{0}^{\infty}(G)$ is a distribution. (4.3) shows that $\langle u, \phi\rangle=0$ if the support of $\phi$ is disjoint from $K$, so $u$ has compact support, when regarded as a member of $\mathscr{D}^{\prime}(G)$. Therefore, we can conclude that $\mathcal{E}^{\prime}(G) \subset \mathscr{D}^{\prime}(G)$. Moreover, 
Theorem 4.1.8. $C_{0}^{\infty}(G)$ is dense in $C^{\infty}(G)$, and $\mathcal{E}^{\prime}(G)$ is dense in $\mathscr{D}^{\prime}(G)$.

Note that the space $\mathcal{S}^{\prime}\left(\mathbb{R}^{n}\right)$ as introduced in Chapter 1 is a further subspace of $\mathscr{D}^{\prime}\left(\mathbb{R}^{n}\right)$, in fact we have as continuous embeddings $\mathcal{E}^{\prime}\left(\mathbb{R}^{n}\right) \subset \mathcal{S}^{\prime}\left(\mathbb{R}^{n}\right) \subset \mathscr{D}^{\prime}\left(\mathbb{R}^{n}\right)$.

The following theorem regarding convolution of distributions is taken from [10]. The convolution of two functions $f$ and $g$ on $\mathbb{R}^{n}$ is defined by

$$
(f * g)(x)=\int f(y) g(x-y) d y=\int f(x-y) g(y) d y, x \in \mathbb{R}^{n},
$$

provided the integral on the right hand side exists, e.g. $f, g \in L^{1}$ or $f \in L^{1}$ and $g \in L^{p}$ as it follows from Young's inequality. Assuming that both functions are continuous and that at least one has compact support, then $f * g$ exists and is continuous, therefore it determines a distribution which can be seen to be

$$
\langle f * g, \varphi\rangle=\iint f(x) g(y) \varphi(x+y) d x d y, \varphi \in C_{0}^{\infty}\left(\mathbb{R}^{n}\right) .
$$

Before we define the convolution of distributions formally we will discuss several situations for the convolution of functions with distributions which we take from $[17]$.

Theorem 4.1.9. For a distribution $u$ and a function $\phi$ the convolution is defined by

$$
(u * \phi)(x):=u(\phi(x-\cdot))
$$

in each of the following situations:

A) $u \in \mathscr{D}^{\prime}(G)$ and $\phi \in C_{0}^{\infty}(G)$;

B) $u \in \mathcal{E}^{\prime}(G)$ and $\phi \in C^{\infty}(G)$;

C) $u \in \mathcal{S}^{\prime}\left(\mathbb{R}^{n}\right)$ and $\phi \in \mathcal{S}\left(\mathbb{R}^{n}\right)$.

Moreover, in each case $u * \phi$ is a $C^{\infty}$-function and we have

$$
\partial^{\alpha}(u * \phi)=\left(\partial^{\alpha} u\right) * \phi=u *\left(\partial^{\alpha} \phi\right)
$$

and for $u \in \mathcal{S}^{\prime}\left(\mathbb{R}^{n}\right), \phi \in \mathcal{S}\left(\mathbb{R}^{n}\right)$, the function $u * \phi$ has at most polynomial growth. 
Formally, we can define the convolution of two distributions $u$ and $v$ by

$$
(u * v)(\varphi):=u *(v * \varphi)
$$

provided one of them has compact support.

We can look at some theory regarding convolution equations which we take from [10]. Given a distribution $k \in \mathcal{E}^{\prime}\left(\mathbb{R}^{n}\right)$ we have a map $K: \mathscr{D}^{\prime}\left(\mathbb{R}^{n}\right) \rightarrow \mathscr{D}^{\prime}\left(\mathbb{R}^{n}\right)$ given by $u \mapsto k * u$. Note that if $k$ does not have compact support, we can still consider the mapping $u \mapsto k * u$ when restricting the domain of the corresponding operator, for example to the set of all $u \in \mathscr{D}^{\prime}\left(\mathbb{R}^{n}\right)$ such that the restriction of $(x, y) \mapsto x+y$ to $\operatorname{supp} k \times \operatorname{supp} u$ is a proper map. When $k$ has compact support, this is of course all of $\mathscr{D}^{\prime}\left(\mathbb{R}^{n}\right)$. If we choose a cut-off function $\rho \in C_{0}^{\infty}\left(\mathbb{R}^{n}\right)$ such that $\rho=1$ on a neighbourhood of the support of $k$ then we can define a linear form $k * u$ on $C_{0}^{\infty}\left(\mathbb{R}^{n}\right)$ by setting

$$
\langle k * u, \phi\rangle=\langle k(x) \otimes u(y), \rho(x) \phi(x+y)\rangle, \phi \in C_{0}^{\infty}\left(\mathbb{R}^{n}\right)
$$

which is the formal representation of (4.4) along with $\rho$ where $\otimes$ denotes the tensor product. For the convolution of $k$ and $u$ the above defines a distribution given by

$$
\begin{aligned}
\langle k * u, \phi\rangle & =\langle u(y),\langle k(x), \phi(x+y)\rangle\rangle \\
& =\langle k(x),\langle u(y), \phi(x+y)\rangle\rangle,
\end{aligned}
$$

where $\phi \in C_{0}^{\infty}\left(\mathbb{R}^{n}\right)$. Note, that the map $K$ commutes with translation. The fundamental solution of the convolution operator $u \mapsto k * u$ is a distribution $E$ such that

$$
K E=k * E=\varepsilon_{0} .
$$

If $E \in D^{\prime}(G)$ and $v \in \mathcal{E}^{\prime}\left(\mathbb{R}^{n}\right)$ then we obtain with $u=E * v$

$$
k * u=(k * E) * v=\varepsilon_{0} * v=v .
$$

Therefore $u=E * v$ is a solution of the convolution equation $k * u=v$, given that $v$ has compact support. We return to convolution equations in the end of this chapter. 
In order to discuss partial differential operators we need a definition which is taken from [18].

Definition 4.1.10. Let $A$ be a finite set of multi-indices, and let $a_{\alpha} \in C^{\infty}(G)$, $\alpha \in A$. Then

$$
P(x, D)=\sum_{\alpha \in A} a_{\alpha}(x) D^{\alpha}
$$

where we denote $D=-i \partial$ and $D^{\alpha}=(-i)^{|\alpha|} \partial^{\alpha}$, is a linear differential operator with $C^{\infty}$ coefficients, defined on $G$.

The order of a differential operator $P$ is defined by $\max \left\{|\alpha|: \alpha \in A, a_{\alpha} \neq 0\right\}$ which we denote by $m$ and we can write

$$
P(x, D)=\sum_{|\alpha| \leq m} a_{\alpha}(x) D^{\alpha}
$$

Moreover, we have

$$
\langle P u, \phi\rangle=\left\langle u,{ }^{t} P \phi\right\rangle, \phi \in C_{0}^{\infty}(G),
$$

where ${ }^{t} P$ is the differential operator known as the formal adjoint or formal transposed of $P$ defined by

$$
{ }^{t} P(x, D) \phi=\sum_{|\alpha| \leq m}(-1)^{\alpha} D^{\alpha}\left(a_{\alpha} \phi\right) .
$$

A differential operator with constant coefficients

$$
P(D)=\sum_{|\alpha| \leq m} a_{\alpha} D^{\alpha}
$$

defined on $\mathbb{R}^{n}$ can be regarded as a convolution operator. If we take $k=P(D) \varepsilon_{0}$ then we obtain

$$
P(D) \varepsilon_{0} * u=\varepsilon_{0} * P(D) u=P(D) u
$$

\subsection{Fundamental Solutions for Partial Differen- tial Operators with Constant Coefficients}

Definition 4.2.1. A fundamental solution for the partial differential operator $P(D)=\sum_{|\alpha| \leq m} a_{\alpha} D^{\alpha}$ with constant complex coefficients $a_{\alpha} \in \mathbb{C}$ is a distribution 
$E \in \mathscr{D}^{\prime}\left(\mathbb{R}^{n}\right)$ such that $P(D) E=\varepsilon_{0}$, where $\varepsilon_{0}$ is the Dirac measure at $0 \in \mathbb{R}^{n}$.

Recall that with $D=-i \partial$ we have $D^{\alpha}=(-i \partial)^{\alpha}=(-i)^{|\alpha|} \partial^{\alpha}$ and from

$$
e^{-i x \cdot \xi} P\left(D_{x}\right) e^{i x \xi}=P(\xi)=\sum_{|\alpha| \leq m} a_{\alpha} \xi^{\alpha}
$$

we deduce that $P(\xi)$ is determined by $P(D)$. Recall that $P(D) u=v$ means for two distributions $u, v \in \mathscr{D}^{\prime}\left(\mathbb{R}^{n}\right)$ that for all $\varphi \in \mathscr{D}\left(\mathbb{R}^{n}\right)$ we have

$$
\langle P(D) u, \varphi\rangle=\left\langle u, P^{t}(D) \varphi\right\rangle=\langle v, \varphi\rangle
$$

where $P^{t}(D)$ is the formal transposed operator

$$
P^{t}(D)=\sum_{|\alpha| \leq m} a_{\alpha}(-1)^{|\alpha|} D^{\alpha}
$$

It is clear that if $E$ is a fundamental solution of $P(D)$ then $E+u, P(D) u=0$ is a further one. In order to understand basic properties of fundamental solutions we first note that for all $u \in \mathscr{D}^{\prime}\left(\mathbb{R}^{n}\right)$ and all $x \in \mathbb{R}^{n}$ the convolution $u * \varepsilon_{x}$ is defined where $\varepsilon_{x}$ is the Dirac measure at $x$. Moreover, if $v \in \mathcal{E}^{\prime}\left(\mathbb{R}^{n}\right)$, i.e. $v$ is a distribution with compact support, then $u * v=v * u$ is well defined. In particular, for a fundamental solution $E$ to $P(D)$ we obtain for $f \in \mathcal{E}^{\prime}\left(\mathbb{R}^{n}\right)$

$$
P(D)(E * f)=(P(D) E) * f=\varepsilon_{0} * f=f,
$$

i.e. $E * f$ solves the equation $P(D) u=f$ in $\mathscr{D}^{\prime}\left(\mathbb{R}^{n}\right)$ for $f \in \mathcal{E}^{\prime}\left(\mathbb{R}^{n}\right)$.

Next we note that differential operators are local and pseudo-local, i.e. for every $u \in \mathscr{D}^{\prime}\left(\mathbb{R}^{n}\right)$ we have

$$
\operatorname{supp} P(D) u \subset \operatorname{supp} u
$$

and

$$
\text { sing supp } P(D) u \subset \operatorname{sing} \operatorname{supp} u \text {. }
$$

It is well known that for two distributions $u_{1}, u_{2} \in \mathscr{D}^{\prime}\left(\mathbb{R}^{n}\right)$ such that at least one has compact support it follows that

$$
\operatorname{supp}\left(u_{1} * u_{2}\right) \subset \operatorname{supp} u_{1}+\operatorname{supp} u_{2}
$$


and

$$
\operatorname{sing} \operatorname{supp}\left(u_{1} * u_{2}\right) \subset \operatorname{sing} \operatorname{supp} u_{1}+\operatorname{sing} \operatorname{supp} u_{2} \text {. }
$$

As a first result of importance to us we may state from [17]

Theorem 4.2.2. If $P(D)$ has a fundamental solution $E$ with the property that sing supp $E=\{0\}$ then for every $u \in \mathscr{D}^{\prime}\left(\mathbb{R}^{n}\right)$ we have

$$
\text { sing supp } P(D) u=\operatorname{sing} \operatorname{supp} u \text {. }
$$

Proof. Since sing supp $P(D) u \subset$ sing supp $u$ it remains to prove that sing supp $u \subset \operatorname{sing} \operatorname{supp} P(D) u$. For $u \in \mathcal{E}^{\prime}\left(\mathbb{R}^{n}\right)$ we find with $u=E * P(D) u$ that

$\operatorname{sing} \operatorname{supp} u=\operatorname{sing} \operatorname{supp} E * P(D) u \subset \operatorname{sing} \operatorname{supp} E+\operatorname{sing} \operatorname{supp} P(D) u$

and therefore sing supp $u \subset\{0\}+\operatorname{sing} \operatorname{supp} P(D) u=\operatorname{sing} \operatorname{supp} P(D) u$. Now for arbitrary $u \in \mathscr{D}^{\prime}\left(\mathbb{R}^{n}\right)$ and $\psi \in C_{0}^{\infty}\left(\mathbb{R}^{n}\right),\left.\psi\right|_{G^{\prime}}=1$ where $G^{\prime} \subset \mathbb{R}^{n}$ is open, we find

$$
\begin{aligned}
G^{\prime} \cap \operatorname{sing} \operatorname{supp} P(D) u & =G^{\prime} \cap \operatorname{sing} \operatorname{supp} P(D)(\psi u) \\
& =G^{\prime} \cap \operatorname{sing} \operatorname{supp}(\psi u)=G^{\prime} \cap \operatorname{sing} \operatorname{supp} u
\end{aligned}
$$

implying the general case.

This result implies for a fundamental solution $E$ of $P(D)$ such that sing supp $E=$ $\{0\}$ that

$$
\operatorname{sing} \operatorname{supp} u=\operatorname{sing} \operatorname{supp} P(D) u=\operatorname{sing} \operatorname{supp} g
$$

if $g \in \mathcal{E}^{\prime}\left(\mathbb{R}^{n}\right)$ and $u=E * g$ is a solution to $P(D) u=g$. The meaning of (4.6) is that the solution $u=E * g$ is a $C^{\infty}$-function in every open set where $g$ is a $C^{\infty}$ function, i.e. (4.6) is a rather sharp regularity result for distributional solutions of $P(D) u=g$. Of course, in general $P(D)$ will not have such a property.

Definition 4.2.3. A differential operator $P(D)$ is called hypoelliptic if for every $u \in \mathscr{D}^{\prime}\left(\mathbb{R}^{n}\right)$ the inclusion

$$
\text { sing supp } u \subset \text { sing supp } P(D) u
$$

holds. 
Corollary 4.2.4. If $P(D)$ has a fundamental solution $E$ such that sing suppE $=$ $\{0\}$ then $P(D)$ is hypoelliptic.

The following famous result due to L.Hörmander gives a characterization of hypoelliptic differential operators with constant coefficients.

Theorem 4.2.5. The differential operator $P(D)$ is hypoelliptic if and only if for every $\alpha \in \mathbb{N}_{0}^{n}$ we have

$$
\frac{\left|P^{(\alpha)}(\xi)\right|}{|P(\xi)|} \leq c|\xi|^{-\sigma|\alpha|},|\xi| \geq \rho
$$

where $c>0, \rho \geq 0$ and $\sigma>0$ are independent of $\alpha$.

Definition 4.2.6. A polynomial satisfying (4.7) is called a hypoelliptic polynomial.

We have not discussed so far whether a fundamental solution exists. This is the content of the Theorem of Malgrange and Ehrenpreis which is taken from [10]:

Theorem 4.2.7. Every partial differential operator with constant coefficients has a fundamental solution.

Corollary 4.2.8. For every partial differential operator $P(D)$ with constant coefficients the differential equation $P(D) u=g$ has for $g \in \mathcal{E}^{\prime}\left(\mathbb{R}^{n}\right)$ in $\mathscr{D}^{\prime}\left(\mathbb{R}^{n}\right)$ a solution.

We need a refinement of the Malgrange-Ehrenpreis theorem. For a polynomial $P(\xi)$ we denote by $\tilde{P}(\xi)$ the term defined by

$$
\tilde{P}^{2}(\xi)=\sum_{\alpha \in \mathbb{N}_{0}^{n}}\left|P^{(\alpha)}(\xi)\right|^{2}
$$

with $P^{(\alpha)}(\xi)=\partial^{\alpha} P(\xi)$. Furthermore, for $1 \leq p \leq \infty$ we introduce

$$
\mathscr{B}_{p, \tilde{P}}\left(\mathbb{R}^{n}\right)=\left\{u \in \mathcal{S}^{\prime}\left(\mathbb{R}^{n}\right) \mid \tilde{P}(\cdot) \hat{u} \in L^{p}\left(\mathbb{R}^{n}\right)\right\}
$$

hence $\mathcal{S}\left(\mathbb{R}^{n}\right) \subset \mathscr{B}_{p, \tilde{P}}\left(\mathbb{R}^{n}\right) \subset \mathcal{S}^{\prime}\left(\mathbb{R}^{n}\right)$ and $\mathscr{B}_{p, \tilde{P}}^{\text {loc }}\left(\mathbb{R}^{n}\right)$ is the space

$$
\mathscr{B}_{p, \tilde{P}}^{l o c}\left(\mathbb{R}^{n}\right)=\left\{u \in \mathscr{D}^{\prime}\left(\mathbb{R}^{n}\right) \mid \varphi u \in \mathscr{B}_{p, \tilde{P}}\left(\mathbb{R}^{n}\right) \text { for every } \varphi \in C_{0}^{\infty}\left(\mathbb{R}^{n}\right\}\right.
$$


Theorem 4.2.9. (L. Hörmander [18]): Every partial differential operator with constant coefficients admits a fundamental solution in $\mathscr{B}_{p, \tilde{P}}^{l o c}\left(\mathbb{R}^{n}\right)$.

It will be useful to state some examples of fundamental solutions.

Theorem 4.2.10. Put $E(x)=(2 \pi)^{-1} \log |x|$ if $x \in \mathbb{R}^{2} \backslash 0$ and for $n>2$

$$
E(x)=\frac{-|x|^{2-n}}{(n-2) c_{n}}, x \in \mathbb{R}^{n} \backslash 0,
$$

where $|x|$ is the Euclidean norm and $c_{n}$ the area of the unit sphere. Then

$$
\Delta E=\sum_{1}^{n} \partial_{j}^{2} E=\varepsilon_{0}
$$

Moreover the Laplacian is an example of a hypoelliptic operator. We note in addition that $\partial_{j} E$ is also generated by a locally integrable function, namely $x \mapsto$ $\frac{x_{j}|x|^{-n}}{c}$, but $\partial_{j} \partial_{k} E$ not.

The following example is with regard to the heat equation in $\mathbb{R}^{n+1}$.

Theorem 4.2.11. Denote the variables in $\mathbb{R}^{n+1}$ by $(x, t) \in \mathbb{R}^{n} \times \mathbb{R}$ and set

$$
E(x, t)=(4 \pi t)^{-\frac{n}{2}} \exp \left(-\frac{|x|^{2}}{4 t}\right), t>0, E(x, t)=0, t \leq 0 .
$$

Then $E$ is locally integrable in $\mathbb{R}^{n+1}, E \in C^{\infty}\left(\mathbb{R}^{n+1} \backslash 0\right)$, and

$$
\left(\frac{\partial}{\partial t}-\Delta_{x}\right) E=\varepsilon_{0}
$$

The heat equation operator is hypoelliptic.

We also give the fundamental solution with regard to the wave equation in $\mathbb{R}$.

Theorem 4.2.12. Denote $E=\frac{1}{2} H(t-x) H(t+x)$ where $H$ is the Heaviside function as defined in Example 4.1.6 and also $E$ is supported in $t \geq|x|$. Then $E$ is the fundamental solution to the wave equation where

$$
\left(\frac{\partial^{2}}{\partial t^{2}}-c^{2} \Delta\right) E=\left(\frac{\partial^{2}}{\partial t^{2}}-c^{2} \sum_{1}^{n} \frac{\partial^{2}}{\partial x_{j}^{2}}\right) E=\varepsilon_{0}
$$

Moreover the wave equation operator is not hypoelliptic. 
Example 4.2.13. On $\mathbb{R}$ define the function $x \mapsto x_{+}$where

$$
x_{+}=x H(x), x \in \mathbb{R},
$$

so that $x_{+}=x$ if $x>0$ and $x_{+}=0$ if $x \leq 0$. Then $\partial x_{+}=H$ and so

$$
\partial^{k}\left(\frac{x_{+}^{k-1}}{(k-1) !}\right)=\varepsilon_{0}, \quad k=1,2, \ldots
$$

If we set

$$
E_{k}=\frac{\left(x_{1}\right)_{+}^{k-1} \ldots\left(x_{n}\right)_{+}^{k-1}}{((k-1) !)^{n}}, x \in \mathbb{R}^{n}
$$

then we have, in $\mathscr{D}^{\prime}\left(\mathbb{R}^{n}\right)$, for $k=1,2, \ldots$,

$$
\left(\partial_{1} \ldots \partial_{n}\right)^{k} E_{k}=\varepsilon_{0}
$$

The results in this chapter are all taken from L.Hörmander [17] and [18], but our presentation is also influenced by [20]. It should be mentioned that the proof of the Malgrange-Ehrenpreis theorem as well as proofs establishing estimates or other results for fundamental solutions for the partial differential operator $P(D)$ make use of the fact that the symbol of $P(D)$ is a polynomial $P(\xi)$ hence an entire function and in addition, in order to study $E * g, g \in \mathcal{E}^{\prime}\left(\mathbb{R}^{n}\right)$, it is used that $\hat{g}$ is an entire function of controlled growth. We refer to Hörmander [17] and in particular [18] for studies of solutions of partial differential equations with constant coefficients using the fundamental solution.

Differential operators with constant coefficients are translation invariant and hence convolution operators. This suggests to study more general convolution equations $K u=k * u=g$ along the lines of the study of partial differential equations with constant coefficients. Such a theory is well developed for $k \in$ $\mathcal{E}^{\prime}\left(\mathbb{R}^{n}\right)$ and again complex analysis of functions of several complex variables becomes the main tool we refer to our previous considerations in this chapter. As a standard reference we refer to Hörmander [18]. As we will see soon, most of the results on fundamental solutions for partial differential operators with constant coefficients and/or convolution equations we cannot apply to our problems.

Consider once more the equation

$$
P(D) E=\varepsilon_{0} .
$$


Since $\varepsilon_{0} \in \mathcal{S}^{\prime}\left(\mathbb{R}^{n}\right)$ and $\hat{\varepsilon}_{0}=(2 \pi)^{-\frac{n}{2}}$ this equation is equivalent to

$$
(P(D) E)^{\wedge}=(2 \pi)^{-\frac{n}{2}}
$$

However for $E \in \mathscr{D}^{\prime}\left(\mathbb{R}^{n}\right) \backslash \mathcal{S}^{\prime}\left(\mathbb{R}^{n}\right)$ we cannot manipulate the left hand side of (4.8) to obtain $P(\cdot) \hat{E}=(2 \pi)^{-\frac{n}{2}}$ and try to find the Fourier inverse of $\hat{E}=(2 \pi)^{-\frac{n}{2}} \frac{1}{P(\cdot)}$. Now let $\psi: \mathbb{R}^{n} \rightarrow \mathbb{C}$ be a continuous function with of at most polynomial growth. As already discussed before (for continuous negative definite functions) we can define on $\mathcal{S}\left(\mathbb{R}^{n}\right)$

$$
\psi(D) u(x):=(2 \pi)^{-\frac{n}{2}} \int_{\mathbb{R}^{n}} e^{i x \xi} \psi(\xi) \hat{u}(\xi) d \xi
$$

with the obvious consequence that

$$
(\psi(D) u)^{\wedge}(\xi)=\psi(\xi) \hat{u}(\xi)
$$

where the right hand side is at least an element in $L^{1}\left(\mathbb{R}^{n}\right) \cap C_{b}\left(\mathbb{R}^{n}\right)$, in fact $\psi(\cdot) \hat{u}$ must decay faster than any polynomial. Defining a fundamental solution to $\psi(D)$ as a distribution $E \in \mathcal{S}^{\prime}\left(\mathbb{R}^{n}\right)$, and not as a distribution $E \in \mathscr{D}^{\prime}\left(\mathbb{R}^{n}\right)$, satisfying

$$
\psi(\xi) \hat{E}(\xi)=(2 \pi)^{-\frac{n}{2}}
$$

makes sense, and depending on the zeroes of $\psi$ we may also use

$$
\hat{E}(\xi)=\frac{1}{(2 \pi)^{\frac{n}{2}} \psi(\xi)} .
$$

If $\frac{1}{\psi(\cdot)} \in \mathcal{S}^{\prime}\left(\mathbb{R}^{n}\right)$ then we can find $E=F^{-1}\left(\frac{1}{(2 \pi)^{\frac{n}{2}} \psi(\cdot)}\right)$ in $\mathcal{S}^{\prime}\left(\mathbb{R}^{n}\right)$ and $(4.9)$ or $\psi(D) E=\varepsilon_{0}$ are satisfied. We do not follow this idea directly, but we want to use more of the properties of $\psi$ in order to get better properties of $E$ "automatically" from the definition. For continuous negative definite functions such an approach is rather satisfying.

\subsection{The Spaces $H^{\psi, s}\left(\mathbb{R}^{n}\right)$ and Related Spaces}

In order to study operators $\psi(D), \psi$ being a continuous negative definite function or a more general function such as a tempered weight function introduced by 
Hörmander, [18], or an even less regular function, we need to introduce an appropriate scale of function spaces defined with the help of $\psi$. Although our definition is along the line of that of classical Bessel potential spaces due to the lack of regularity and homogeneity of continuous negative definite functions certain new aspects enter into our considerations. The space $H^{\psi, s}\left(\mathbb{R}^{n}\right)$ had been first introduced and studied in [19], the standard reference in [20] and extension to an $L^{p}$-theory is given in [9]. In the end of this chapter we will extend our considerations to more general functions than continuous negative definite functions. At the moment, in this chapter if not stated otherwise $\psi: \mathbb{R}^{n} \rightarrow \mathbb{R}$ is always a real-valued continuous negative definite function, hence it is nonnegative and satisfies Peetre's inequality

$$
\frac{1+\psi(\xi)}{1+\psi(\eta)} \leq 2(1+\psi(\xi-\eta))
$$

for all $\xi, \eta \in \mathbb{R}^{n}$ which is a consequence of the "triangle inequality"

$$
\psi^{\frac{1}{2}}(\xi+\eta) \leq \psi^{\frac{1}{2}}(\xi)+\psi^{\frac{1}{2}}(\eta) .
$$

We note once more that if $\psi: \mathbb{R}^{n} \rightarrow \mathbb{R}$ is a continuous negative definite function such that $\psi(0)=0$ and that $\psi(\xi)=0$ implies $\xi=0$, then $\psi^{\frac{1}{2}}(\xi-\eta)$ defines a metric on $\mathbb{R}^{n}$ which generates the Euclidean topology if and only if

$$
\lim _{|\xi| \rightarrow \infty} \psi(\xi)>0
$$

see [23] and our discussion in section 3.1. We start with

Definition 4.3.1. Let $\psi: \mathbb{R}^{n} \rightarrow \mathbb{R}$ be a continuous negative definite function and $s \in \mathbb{R}$. We introduce the norm

$$
\|u\|_{\psi, s}:=\left\|(1+\psi(\cdot))^{\frac{s}{2}} \hat{u}\right\|_{L^{2}}
$$

and we introduce the space

$$
H^{\psi, s}\left(\mathbb{R}^{n}\right)=\left\{u \in \mathcal{S}^{\prime}\left(\mathbb{R}^{n}\right) \mid\|u\|_{\psi, s}<\infty\right\}
$$


Since $\left\|(1+\psi(\cdot))^{\frac{s}{2}} \hat{u}\right\|_{L^{2}}<\infty$ implies $(1+\psi(\cdot))^{\frac{s}{2}} \hat{u} \in L^{2}\left(\mathbb{R}^{n}\right)$ it follows that $F^{-1}\left((1+\psi(\cdot))^{\frac{s}{2}} \hat{u}\right) \in L^{2}\left(\mathbb{R}^{n}\right)$. Moreover, since $\psi(\xi) \geq 0$ it follows that $\mathcal{S}\left(\mathbb{R}^{n}\right) \subset$ $H^{\psi, s}\left(\mathbb{R}^{n}\right)$ for every $s \in \mathbb{R}$ and for $u \in \mathcal{S}\left(\mathbb{R}^{n}\right)$ we have

$$
\|u\|_{\psi, s}^{2}=\int_{\mathbb{R}^{n}}(1+\psi(\xi))^{s}|\hat{u}(\xi)|^{2} d \xi .
$$

We observe that $H^{\psi, s}\left(\mathbb{R}^{n}\right)$ is isometrically isomorphic to the weighted space $L^{2}\left(\mathbb{R}^{n} ;(1+\psi(\cdot))^{\frac{s}{2}} \lambda^{(n)}\right)$, we just need to consider the mapping $u \mapsto(1+\psi(\cdot))^{\frac{s}{2}} \hat{u}$ and apply a variant of Plancherel's theorem. This also gives the density of $\mathcal{S}\left(\mathbb{R}^{n}\right)$ in $H^{\psi, s}\left(\mathbb{R}^{n}\right)$. Hence we have proved

Theorem 4.3.2. The space $H^{\psi, s}\left(\mathbb{R}^{n}\right)$ is a Hilbert space with dense subspace $\mathcal{S}\left(\mathbb{R}^{n}\right)$ and the scalar product given by

$$
(u, v)_{\psi, s}:=\left((1+\psi(\cdot))^{\frac{s}{2}} \hat{u},(1+\psi(\cdot))^{\frac{s}{2}} \hat{v}\right)_{L^{2}}
$$

Note that for $u, v \in \mathcal{S}\left(\mathbb{R}^{n}\right)$ we can rewrite (4.10) as

$$
(u, v)_{\psi, s}:=\int_{\mathbb{R}^{n}}(1+\psi(\xi))^{s} \hat{u}(\xi) \overline{\hat{v}(\xi)} d \xi .
$$

In the case where $\psi(\xi)=|\xi|^{2}$ we have of course

$$
\|u\|_{s}=\|u\|_{\psi, s} \text { and } H^{s}\left(\mathbb{R}^{n}\right)=H^{\psi, s}\left(\mathbb{R}^{n}\right)
$$

where $H^{s}\left(\mathbb{R}^{n}\right)$ is the classical Bessel potential space which for $s \in \mathbb{N}_{0}$ coincides with the Sobolev space $W^{s, 2}\left(\mathbb{R}^{n}\right)$. As elementary results we state

Theorem 4.3.3. Let $\psi: \mathbb{R}^{n} \rightarrow \mathbb{R}$ be a continuous negative definite function. The estimate

$$
\|u\|_{\psi, t} \leq\|u\|_{\psi, s}
$$

holds for $s \geq t$.

Proof. The proof follows easily from the fact that for $s \geq t$ and for $u \in \mathcal{S}\left(\mathbb{R}^{n}\right)$ we have

$$
\begin{aligned}
\|u\|_{\psi, t}^{2} & =\int_{\mathbb{R}^{n}}(1+\psi(\xi))^{t}|\hat{u}(\xi)|^{2} d \xi \\
& \leq \int_{\mathbb{R}^{n}}(1+\psi(\xi))^{s}|\hat{u}(\xi)|^{2} d \xi \\
& =\|u\|_{\psi, s}^{2} .
\end{aligned}
$$


A consequence of the previous two theorems is the following.

Corollary 4.3.4. $H^{\psi, s}\left(\mathbb{R}^{n}\right) \subset H^{\psi, t}\left(\mathbb{R}^{n}\right)$ if $s \geq t$, and the inclusion mapping is continuous.

The following result is analogue to the classical Sobolev embedding theorem.

Proposition 4.3.5. Let $\psi: \mathbb{R}^{n} \rightarrow \mathbb{R}$ be a continuous negative definite function satisfying

$$
\psi(\xi) \geq c_{0}|\xi|^{\rho_{0}}
$$

for $c_{0}, \rho_{0}>0$ and all $\xi \in \mathbb{R}^{n},|\xi| \geq R$. Then we have for all $u \in H^{\psi, s}\left(\mathbb{R}^{n}\right)$, $s>\frac{n}{\rho_{0}}$,

$$
\|u\|_{\infty} \leq c_{\psi, s, n}\|u\|_{\psi, s}
$$

and $u \in C_{\infty}\left(\mathbb{R}^{n}\right)$.

Proof. For $u \in \mathcal{S}\left(\mathbb{R}^{n}\right)$,

$$
\begin{aligned}
|u(x)| & =\left|\left(F^{-1} F u\right)(x)\right| \\
& =\left|(2 \pi)^{\frac{-n}{2}} \int e^{i x \xi} \frac{(1+\psi(\xi))^{\frac{s}{2}}}{(1+\psi(\xi))^{\frac{s}{2}}} \hat{u}(\xi) d \xi\right| \\
& \leq(2 \pi)^{\frac{-n}{2}} \int \frac{(1+\psi(\xi))^{\frac{s}{2}}}{(1+\psi(\xi))^{\frac{s}{2}}}|\hat{u}(\xi)| d \xi \\
& \leq(2 \pi)^{\frac{-n}{2}}\left(\int \frac{1}{(1+\psi(\xi))^{s}} d \xi\right)^{\frac{1}{2}}\left(\int(1+\psi(\xi))^{s}|\hat{u}(\xi)|^{2} d \xi\right)^{\frac{1}{2}} \\
& =(2 \pi)^{\frac{-n}{2}}\left(\int \frac{1}{(1+\psi(\xi))^{s}} d \xi\right)^{\frac{1}{2}}\|u\|_{\psi, s} .
\end{aligned}
$$

For the above to hold we need

$$
\left(\int \frac{1}{(1+\psi(\xi))^{s}} d \xi\right)<\infty .
$$

Firstly we split the integral into two parts.

$$
\begin{aligned}
\left(\int \frac{1}{(1+\psi(\xi))^{s}} d \xi\right) & =\left(\int_{\overline{B_{R}(0)}}+\int_{\mathbb{R}^{n} \backslash \overline{B_{R}(0)}}\right) \frac{1}{(1+\psi(\xi))^{s}} d \xi \\
& =I_{1}(s, R)+I_{2}(s, R) .
\end{aligned}
$$


Clearly, $I_{1}(s, R)<\infty$ since it is the integral of a continuous function over a compact set. $I_{2}$ needs a little more work.

$$
I_{2}=\int_{\mathbb{R}^{n} \backslash \overline{B_{R}(0)}} \frac{1}{(1+\psi(\xi))^{s}} d \xi \leq \int_{\mathbb{R}^{n} \backslash \overline{B_{R}(0)}} \frac{1}{\psi(\xi)^{s}} d \xi
$$

If we use the assumption,

$$
\frac{1}{\psi(\xi)} \leq \frac{1}{c_{0}}|\xi|^{-\rho_{0}} \text { for all }|\xi| \geq R
$$

we have

$$
I_{2} \leq \frac{1}{c_{0}^{s}} \int_{\mathbb{R}^{n} \backslash \overline{B_{r}(0)}}|\xi|^{-\rho_{0} s} d \xi=\frac{w_{n}}{c_{0}^{s}} \int_{R}^{\infty} r^{-\rho_{0} s} r^{n-1} d r .
$$

Therefore, we need

$$
\int_{R}^{\infty} r^{n-1-\rho_{0} s} d r<\infty \Longleftrightarrow n-1-\rho_{0} s<-1 \Longleftrightarrow s>\frac{n}{\rho_{0}}
$$

We conclude that taking the condition that

$$
\psi(\xi) \geq c_{0}|\xi|^{\rho_{0}}, \quad c_{0}, \rho_{0}>0
$$

for all $\xi \in \mathbb{R}^{n},|\xi| \geq R$, if $s>\frac{n}{\rho_{0}}$ then for all $u \in \mathcal{S}\left(\mathbb{R}^{n}\right)$

$$
\|u\|_{\infty}=\sup _{x \in \mathbb{R}^{n}}|u(x)| \leq(c+\tilde{c})\|u\|_{\psi, s} .
$$

Since, $\mathcal{S}$ is dense in $C_{\infty}\left(\mathbb{R}^{n}\right)$ and $H^{\psi, s}\left(\mathbb{R}^{n}\right)$, implies $(4.11)$ for all $u \in H^{\psi, s}\left(\mathbb{R}^{n}\right)$.

Moreover we have the following result.

Proposition 4.3.6. Let $\psi: \mathbb{R}^{n} \rightarrow \mathbb{R}$ be a continuous negative definite function. If $\left(1+|\xi|^{2}\right)^{\frac{\rho_{0}}{2}} \leq c(1+\psi(\xi))$, then

$$
\left|\partial^{\alpha} u(x)\right| \leq c\|u\|_{\psi, k+\frac{2|\alpha|}{\rho_{0}}}
$$

for all $u \in H^{\psi, k+\frac{2|\alpha|}{\rho_{0}}}\left(\mathbb{R}^{n}\right)$. In particular $\partial^{\alpha} u \in C_{\infty}\left(\mathbb{R}^{n}\right)$ for $k>\frac{n}{\rho_{0}}$ where $k=k\left(n, \rho_{0}\right)$. 
Proof. For $u \in \mathcal{S}\left(\mathbb{R}^{n}\right)$, we have

$$
\begin{aligned}
\left|\partial^{\alpha} u(x)\right| & =\left|F^{-1} F\left(\partial^{\alpha} u\right)\right| \\
& \leq(2 \pi)^{-\frac{n}{2}} \int_{\mathbb{R}^{n}}\left|F\left(\partial^{\alpha} u\right)(\xi)\right| d \xi \\
& =(2 \pi)^{-\frac{n}{2}} \int_{\mathbb{R}^{n}}\left|\xi^{\alpha} \hat{u}(\xi)\right| d \xi \\
& \leq(2 \pi)^{-\frac{n}{2}} \int_{\mathbb{R}^{n}}\left(1+|\xi|^{2}\right)^{\frac{|\alpha|}{2}}|\hat{u}(\xi)| d \xi \\
& \leq(2 \pi)^{-\frac{n}{2}} c \int_{\mathbb{R}^{n}}(1+\psi(\xi))^{\frac{|\alpha|}{\rho_{0}}}|\hat{u}(\xi)| d \xi \\
& \leq(2 \pi)^{-\frac{n}{2}}\left(\int_{\mathbb{R}^{n}} \frac{1}{(1+\psi(\xi))^{k}} d \xi\right)^{\frac{1}{2}}\left(\int_{\mathbb{R}^{n}}(1+\psi(\xi))^{\frac{2|\alpha|}{\rho_{0}}+k}|\hat{u}(\xi)|^{2} d \xi\right)^{\frac{1}{2}} \\
& \leq c\|u\|_{\psi, k+\frac{2|\alpha|}{\rho_{0}}}
\end{aligned}
$$

where we have used the Cauchy-Schwarz inequality. Note we also have

$$
\left(\int_{\mathbb{R}^{n}} \frac{1}{(1+\psi(\xi))^{k}} d \xi\right)^{\frac{1}{2}}<\infty
$$

for $k>\frac{n}{\rho_{0}}$ which holds from the previous proposition.

From the previous two propositions we can conclude that we have an embedding for the intersection of all $H^{\psi, s}\left(\mathbb{R}^{n}\right)$ into $C^{\infty}\left(\mathbb{R}^{n}\right)$, i.e. $\bigcap_{s \in \mathbb{R}} H^{\psi, s}\left(\mathbb{R}^{n}\right) \hookrightarrow C_{\infty}^{\infty}\left(\mathbb{R}^{n}\right)$.

We also know that $H^{\psi, s}\left(\mathbb{R}^{n}\right) \subset \mathcal{S}^{\prime}\left(\mathbb{R}^{n}\right)$, therefore from the above results we can conclude that we also have $\bigcup_{s \in \mathbb{R}} H^{\psi, s}\left(\mathbb{R}^{n}\right) \subset \mathcal{S}^{\prime}\left(\mathbb{R}^{n}\right)$. This is summarised in the following corollary.

Corollary 4.3.7. Given a continuous negative definite function $\psi: \mathbb{R}^{n} \rightarrow \mathbb{R}$ which satisfies

$$
\psi(\xi) \geq c_{0}|\xi|^{\rho}
$$

for $c_{0}, \rho_{0}>0$ and all $\xi \in \mathbb{R}^{n},|\xi| \geq R$, then the estimates in Proposition 4.3.5 and 4.3.6 hold and we can conclude that

$$
\bigcap_{s \in \mathbb{R}} H^{\psi, s} \hookrightarrow C_{\infty}^{\infty}
$$

The proof of Proposition 4.3.5 and Proposition 4.3.6 will work under the assumption $\frac{1}{(1+\psi(\cdot))^{k}} \in L^{1}\left(\mathbb{R}^{n}\right)$. In fact we essentially use the classical proof of Sobolev's embedding theorem combined with the following useful observation. 
Proposition 4.3.8. Let $\psi_{1}, \psi_{2}: \mathbb{R}^{n} \rightarrow \mathbb{R}$ be two continuous negative definite functions such that for some $\kappa_{0}>0$ we have $\left(1+\psi_{1}(\xi)\right) \leq \kappa_{0}\left(1+\psi_{2}(\xi)\right)$ for all $\xi \in \mathbb{R}^{n}$. Then we have for all $s$ the estimate

$$
\|u\|_{\psi_{1}, s} \leq \kappa_{0}^{\frac{s}{2}}\|u\|_{\psi_{2}, s}
$$

and $H^{\psi_{2}, s}\left(\mathbb{R}^{n}\right)$ is continuously embedded into $H^{\psi_{1}, s}\left(\mathbb{R}^{n}\right)$.

Proof. For $u \in \mathcal{S}\left(\mathbb{R}^{n}\right)$ we find

$$
\begin{aligned}
\|u\|_{\psi_{1}, s}^{2} & =\int_{\mathbb{R}^{n}}\left(1+\psi_{1}(\xi)\right)^{s}|\hat{u}(\xi)|^{2} d \xi \\
& \leq \kappa_{0} \int_{\mathbb{R}^{n}}\left(1+\psi_{2}(\xi)\right)^{s}|\hat{u}(\xi)|^{2} d \xi=\kappa_{0}^{s}\|u\|_{\psi_{2}, s}^{2}
\end{aligned}
$$

or $\|u\|_{\psi_{1}, s} \leq \kappa_{0}^{\frac{s}{2}}\|u\|_{\psi_{2}, s}$.

Finally we want to characterize the dual space of $H^{\psi, s}\left(\mathbb{R}^{n}\right)$. We can construct a pairing of $H^{\psi,-s}\left(\mathbb{R}^{n}\right)$ and $H^{\psi, s}\left(\mathbb{R}^{n}\right)$. Let $u \in \mathcal{S}\left(\mathbb{R}^{n}\right) \subset \mathcal{S}^{\prime}\left(\mathbb{R}^{n}\right)$ be a distribution and $\phi \in \mathcal{S}\left(\mathbb{R}^{n}\right)$ a test function. Then, using Parseval's identity, we obtain

$$
\begin{aligned}
\langle u, \phi\rangle & =\int u(x) \phi(x) d x \\
& =\int \hat{u}(\xi) \hat{\phi}(-\xi) d \xi \\
& =\int(1+\psi(\xi))^{\frac{-s}{2}} \hat{u}(\xi)(1+\psi(\xi))^{\frac{s}{2}} \hat{\phi}(-\xi) d \xi
\end{aligned}
$$

Therefore, we conclude from Cauchy-Schwarz's inequality that

$$
|\langle u, \phi\rangle| \leq\|u\|_{H^{\psi,-s}}\|\phi\|_{H^{\psi, s}} .
$$

By the density of $\mathcal{S}\left(\mathbb{R}^{n}\right)$ in $\mathcal{S}^{\prime}\left(\mathbb{R}^{n}\right)$, we have a pairing of $H^{\psi,-s}\left(\mathbb{R}^{n}\right)$ and $H^{\psi, s}\left(\mathbb{R}^{n}\right)$, i.e. by Theorem 4.3.2, we can extend $\langle u, \phi\rangle$ to a bilinear form on $H^{\psi,-s}\left(\mathbb{R}^{n}\right) \times$ $H^{\psi, s}\left(\mathbb{R}^{n}\right)$,

$$
\langle u, v\rangle=(2 \pi)^{-n} \int_{\mathbb{R}^{n}} \hat{u}(\xi) \hat{v}(-\xi) d \xi, u \in H^{\psi,-s}\left(\mathbb{R}^{n}\right), \quad v \in H^{\psi, s}\left(\mathbb{R}^{n}\right)
$$


which is continuous since

$$
|\langle u, v\rangle| \leq\|u\|_{\psi,-s}\|v\|_{\psi, s}
$$

The bilinear form above turns $H^{\psi, s}$ and $H^{\psi,-s}$ into the dual of each other which we see in more detail in the following theorem, the proof of which follows the ideas of the analogous result for the space $H^{s}\left(\mathbb{R}^{n}\right)$, see [10], we refer also to [9].

Theorem 4.3.9. The pairing (4.12) gives an isometric isomorphism of $H^{\psi,-s}\left(\mathbb{R}^{n}\right)$ and $\left(H^{\psi, s}\left(\mathbb{R}^{n}\right)\right)^{*}$, the dual of $H^{\psi, s}\left(\mathbb{R}^{n}\right)$.

Proof. From (4.13) we have for fixed $u \in H^{\psi,-s}\left(\mathbb{R}^{n}\right)$, that $v \mapsto\langle u, v\rangle$ is a continuous linear form on $H^{\psi, s}\left(\mathbb{R}^{n}\right)$ whose norm does not exceed $\|u\|_{H^{\psi,-s}}$. Taking

$$
v=v_{0}=F^{-1}\left((1+\psi(\xi))^{-s} \hat{u}\right) \in H^{\psi, s}\left(\mathbb{R}^{n}\right)
$$

we obtain $\left\langle u, v_{0}\right\rangle=\|u\|_{H^{\psi,-s}}$. Hence the norm $v \mapsto\langle u, v\rangle$ is equal to $\|u\|_{H^{\psi,-s}}$. Thus we obtain an isometry $H_{\psi,-s}\left(\mathbb{R}^{n}\right) \rightarrow\left(H^{\psi, s}\left(\mathbb{R}^{n}\right)\right)^{*}$. In order to prove that we have an isomorphism, we need to prove that the isometry is surjective. Firstly, we let $u^{*} \in\left(H^{\psi, s}\left(\mathbb{R}^{n}\right)\right)^{*}$. Then, by the Riesz representation theorem, Theorem 2.1.13, we have for some $w \in H^{\psi, s}\left(\mathbb{R}^{n}\right)$,

$$
u^{*}(v)=(v, w)_{s}=\int(1+\psi(\xi))^{s} \hat{v}(\xi) \overline{\hat{w}(\xi)} d \xi
$$

where we have equipped $H^{\psi, s}\left(\mathbb{R}^{n}\right)$ with its inner product.

If we set $u \in F^{-1}\left((1+\psi(\xi))^{s} \overline{\hat{w}(-\xi)}\right)$ then $u \in H^{\psi,-s}\left(\mathbb{R}^{n}\right)$ and $u^{*}(v)=\langle u, v\rangle$ for all $v \in H^{\psi, s}\left(\mathbb{R}^{n}\right)$. Therefore, we have proven that we have an isomorphism. 
We summarize the following dual pairings we have encountered so far:

$$
\begin{gathered}
\mathscr{D}\left(\mathbb{R}^{n}\right)=C_{0}^{\infty}\left(\mathbb{R}^{n}\right) \text { and } \mathscr{D}^{\prime}\left(\mathbb{R}^{n}\right) ; \\
\mathcal{S}\left(\mathbb{R}^{n}\right) \text { and } \mathcal{S}^{\prime}\left(\mathbb{R}^{n}\right) ; \\
\mathcal{E}\left(\mathbb{R}^{n}\right)=C^{\infty}\left(\mathbb{R}^{n}\right) \text { and } \mathcal{E}^{\prime}\left(\mathbb{R}^{n}\right) ; \\
H^{\psi, s}\left(\mathbb{R}^{n}\right) \text { and } H^{\psi,-s}\left(\mathbb{R}^{n}\right) .
\end{gathered}
$$

In the case of $\mathscr{D}\left(\mathbb{R}^{n}\right)$ and $\mathscr{D}^{\prime}\left(\mathbb{R}^{n}\right)$ as well as $\mathcal{E}\left(\mathbb{R}^{n}\right)$ and $\mathcal{E}^{\prime}\left(\mathbb{R}^{n}\right)$ we may replace $\mathbb{R}^{n}$ by an open set $G \subset \mathbb{R}^{n}$.

Note that we can replace in the definition of $H^{\psi, s}\left(\mathbb{R}^{n}\right)$ the continuous negative definite function $\psi: \mathbb{R}^{n} \rightarrow \mathbb{R}$ by a continuous, non-negative function $k: \mathbb{R}^{n} \rightarrow \mathbb{R}$ which has at most polynomial growth, i.e. $k(\xi) \leq c\left(1+|\xi|^{2}\right)^{\frac{\sigma_{0}}{2}}$ for some $\sigma_{0} \geq 0$ and all $\xi \in \mathbb{R}^{n}$. In order to get "good" results $k$ should satisfy Peetre's inequality, i.e. $\frac{1+k(\xi)}{1+k(\eta)} \leq \kappa_{0}(1+k(\xi-\eta))$ for all $\xi, \eta \in \mathbb{R}^{n}$ or a similar type of estimate such as temperate weight functions in the sense of Hörmander, see [18]. In particular we can include higher powers of continuous negative definite functions.

\subsection{On Fundamental Solutions of Pseudo-Differential Operators with Negative Definite Symbols}

We have seen that the generator $A$ of a $L^{2}$ sub-Markovian semigroup or of a Feller semigroup induced by a convolution semigroup $\left(\mu_{t}\right)_{t \geq 0}$ on $\mathbb{R}^{n}$ with associated continuous negative definite function $\psi: \mathbb{R}^{n} \rightarrow \mathbb{C}$ is given on $\mathcal{S}\left(\mathbb{R}^{n}\right)$ by

$$
-A u(x)=\psi(D) u(x)=(2 \pi)^{-\frac{n}{2}} \int_{\mathbb{R}^{n}} e^{i x \xi} \psi(\xi) \hat{u}(\xi) d \xi
$$

i.e. $\psi(D)$ is a pseudo-differential operator with symbol $\psi$. It is natural to study $\psi(D)$ in its own right, say as an object in the theory of (pseudo-) differential operators and the first and most natural question is whether we can extend $\psi(D)$ from $\mathcal{S}\left(\mathbb{R}^{n}\right)$ to a larger space. Having in mind the theory of distributions, the 
first idea must be to extend $\psi(D)$ by duality to $\mathcal{S}^{\prime}\left(\mathbb{R}^{n}\right)$. For $u, v \in \mathcal{S}\left(\mathbb{R}^{n}\right)$ we find

$$
\begin{aligned}
\langle\psi(D) u, v\rangle & =\int_{\mathbb{R}^{n}} \psi(D) u(x) v(x) d x=(\psi(D) u, \bar{v})_{L^{2}} \\
& =\int_{\mathbb{R}^{n}} \psi(\xi) \hat{u}(\xi) \overline{(\bar{v})^{\wedge}}(\xi) d \xi \\
& =\int_{\mathbb{R}^{n}} \hat{u}(\xi) \overline{\overline{\psi(\xi)}(\bar{v})^{\wedge}(\xi)} d \xi \\
& =(u, \bar{\psi}(D) \bar{v})_{L^{2}} \\
& =\int_{\mathbb{R}^{n}} u(x) \overline{(\bar{\psi}(D) \bar{v})}(x) d x \\
& =\left\langle u, F^{-1}\left(\psi(\cdot) F^{-1} v\right)\right\rangle,
\end{aligned}
$$

or with a slight change of the standard approach

$$
\begin{aligned}
\langle\psi(D) u, \bar{v}\rangle & =(\psi(D) u, v)_{L^{2}} \\
& =\int_{\mathbb{R}^{n}} \psi(D) u(x) \overline{v(x)} d x \\
& =\int_{\mathbb{R}^{n}} \hat{u}(\xi) \psi(\xi) \overline{\hat{v}(\xi)} d \xi \\
& =\int_{\mathbb{R}^{n}} \hat{u}(\xi) \overline{\overline{\psi(\xi)} \hat{v}(\xi)} d \xi \\
& =(u, \bar{\psi}(D) v)_{L^{2}}=\langle u, \overline{\bar{\psi}(D) v} .
\end{aligned}
$$

However for an arbitrary continuous negative definite function $\bar{\psi} \hat{v}$ or $\psi F^{-1} v$ do not belong to $\mathcal{S}\left(\mathbb{R}^{n}\right)$. Thus, both suggested formulae, either

$$
\langle\psi(D) u, v\rangle:=\left\langle u, F^{-1}\left(\psi(\cdot) F^{-1} v\right)\right\rangle
$$

or

$$
\langle\psi(D) u, v\rangle:=\langle u, \overline{\bar{\psi}(D) v\rangle}
$$

in general do not make sense for $u \in \mathcal{S}^{\prime}\left(\mathbb{R}^{n}\right)$ and $v \in \mathcal{S}\left(\mathbb{R}^{n}\right)$. Since with $\psi$ also $\bar{\psi}$ is a continuous negative definite function, and with $\hat{v}$ also $v$ belongs to $\mathcal{S}\left(\mathbb{R}^{n}\right)$, we study for a moment the term $\psi v$. In general $\psi v$ is not differentiable but just continuous. However, since $\psi$ has at most quadratic growth, $\psi v$ decays faster than any polynomial, hence is an element of $L^{1}\left(\mathbb{R}^{n}\right) \cap C_{b}\left(\mathbb{R}^{n}\right)$, and the same 
holds for $\psi \hat{v}$. For $u \in \mathcal{S}^{\prime}\left(\mathbb{R}^{n}\right), \psi u$ is not defined since the natural definition must be $\langle\psi u, v\rangle:=\langle u, \psi v\rangle$ for all $v \in \mathcal{S}\left(\mathbb{R}^{n}\right)$ which however requires $\psi v \in \mathcal{S}\left(\mathbb{R}^{n}\right)$. Moreover, even if $\psi u$ could be defined, for example if $\psi \in C^{\infty}\left(\mathbb{R}^{n}\right)$ and is a continuous negative definite function, compare with the growth of $\partial^{\alpha} \psi$ in (1.6), the equation $\psi \hat{u}=\hat{g}$ leads to problems for a function $\psi$ with a zero at, say $\xi=0$. It is known that for every polynomial $P(\xi)$ considered as element in $\mathcal{S}^{\prime}\left(\mathbb{R}^{n}\right)$ the Fourier transform in $\mathcal{S}^{\prime}\left(\mathbb{R}^{n}\right)$ is supported at 0 . Thus $\psi \hat{u}=0$ admits all polynomials at zero and therefore if $\psi \hat{E}=(2 \pi)^{-\frac{n}{2}}$ for some $E \in \mathcal{S}^{\prime}\left(\mathbb{R}^{n}\right)$ all elements $E+P \in \mathcal{S}^{\prime}\left(\mathbb{R}^{n}\right), P$ a polynomial satisfy the same equation! The main conclusion of these considerations is that it is not a good idea to search for a fundamental solution for $\psi(D)$ by trying to invert in $\mathcal{S}^{\prime}\left(\mathbb{R}^{n}\right)$ the equation $\psi(\cdot) \hat{E}=(2 \pi)^{-\frac{n}{2}}$. The situation changes dramatically if we require $\psi \hat{E}$ to be a measurable function. In this case $\psi \hat{E}$ is only defined almost everywhere and isolated zeroes of $\psi$, in fact at set of zeroes having measure zero, does not affect the equation $\psi \hat{E}=(2 \pi)^{-\frac{n}{2}}$ to be interpreted as an equation to hold almost everywhere (with respect to the Lebesgue measure). Now the spaces $H^{\psi, s}\left(\mathbb{R}^{n}\right)$ come to our help. The first observation of importance is

Theorem 4.4.1. For a continuous negative definite function $\psi: \mathbb{R}^{n} \rightarrow \mathbb{R}$, all $s \in \mathbb{R}$ and all $u \in \mathcal{S}\left(\mathbb{R}^{n}\right)$ we have the estimate

$$
\|\psi(D) u\|_{\psi, s} \leq\|u\|_{\psi, s+2},
$$

and $\psi(D)$ extends to a bounded linear operator from $H^{\psi, s+2}\left(\mathbb{R}^{n}\right)$ to $H^{\psi, s}\left(\mathbb{R}^{n}\right)$.

Proof. For $u \in \mathcal{S}\left(\mathbb{R}^{n}\right)$ we note

$$
\begin{aligned}
\|\psi(D) u\|_{\psi, s}^{2} & =\int_{\mathbb{R}^{n}}(1+\psi(\xi))^{s} \psi^{2}(\xi)|\hat{u}(\xi)|^{2} d \xi \\
& \leq \int_{\mathbb{R}^{n}}(1+\psi(\xi))^{s+2}|\hat{u}(\xi)|^{2} d \xi \\
& =\|u\|_{\psi, s+2}^{2} .
\end{aligned}
$$

The second part of the theorem follows from the fact that $\mathcal{S}\left(\mathbb{R}^{n}\right)$ is dense in $H^{\psi, s+2}\left(\mathbb{R}^{n}\right)$. 
In the following we will denote by $\psi(D)$ the extension of the originally defined operator $\psi(D): \mathcal{S}\left(\mathbb{R}^{n}\right) \rightarrow \mathcal{S}\left(\mathbb{R}^{n}\right)$ to any of the space $H^{\psi, s+2}\left(\mathbb{R}^{n}\right)$.

Remark 4.4.2. It is possible to introduce the space $H^{\psi, s}\left(\mathbb{R}^{n}\right)$ for complex-valued continuous negative definite functions $\psi: \mathbb{R}^{n} \rightarrow \mathbb{C}$ by taking as norm the expression

$$
\|u\|_{\psi, s}^{2}:=\int_{\mathbb{R}^{n}}\left(1+|\psi(\xi)|^{2}\right)^{s}|\hat{u}(\xi)|^{2} d \xi
$$

see [20]. Since our main focus will be on real-valued continuous negative definite functions we restrict ourselves also in Theorem 4.4 .1 to real-valued symbols. However, if $\psi: \mathbb{R}^{n} \rightarrow \mathbb{C}$ satisfies the sector condition

$$
|\operatorname{Im} \psi(\xi)| \leq \kappa_{0} \operatorname{Re} \psi(\xi)
$$

for all $\xi \in \mathbb{R}^{n},|\xi| \geq R \geq 0$, the operator $\psi(D)$ is continuous for every $s \in \mathbb{R}$

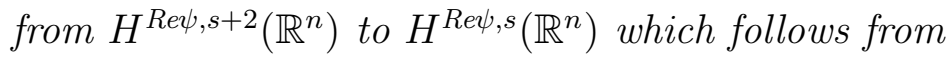

$$
\begin{aligned}
|\psi(\xi)|^{2} & =(\operatorname{Re} \psi(\xi))^{2}+(\operatorname{Im} \psi(\xi))^{2} \\
& \leq\left(1+\kappa_{0}^{2}\right)(\operatorname{Re} \psi(\xi))^{2}
\end{aligned}
$$

implying

$$
\begin{aligned}
\|\psi(D) u\|_{R e \psi, s}^{2} & =\int_{\mathbb{R}^{n}}(1+\operatorname{Re} \psi(\xi))^{2}|\psi(\xi)|^{2}|\hat{u}(\xi)|^{2} d \xi \\
& \leq\left(1+\kappa_{0}^{2}\right) \int_{\mathbb{R}^{n}}(1+\operatorname{Re} \psi(\xi))^{s+2}|\hat{u}(\xi)|^{2} d \xi
\end{aligned}
$$

Theorem 4.4.1 tells us in particular that for a continuous negative definite function $\psi: \mathbb{R}^{n} \rightarrow \mathbb{R}$ the term $\psi(\cdot) \hat{u}$ belongs to $L^{2}\left(\mathbb{R}^{n}\right)$ if $u \in H^{\psi, 2}\left(\mathbb{R}^{n}\right)$ and that $\psi(\cdot) \hat{u}$ is measurable if $u \in H^{\psi, s+2}\left(\mathbb{R}^{n}\right), s \in \mathbb{R}$. Indeed $\psi(D) u \in H^{\psi, s}\left(\mathbb{R}^{n}\right)$ means that $(1+\psi(\cdot))^{\frac{s}{2}} \psi(\cdot) \hat{u}(\cdot)$ belongs to $L^{2}\left(\mathbb{R}^{n}\right)$, hence it is measurable and since $(1+\psi(\cdot))^{-\frac{s}{2}}$ is measurable it follows that $\psi(\cdot) \hat{u}(\cdot)$ is measurable and $\psi \hat{u}=(2 \pi)^{-\frac{n}{2}}$ is an equality of measurable functions. In general $\psi$ will have zeroes, more precisely the most interesting case for us is the case where $\psi(\xi)=0$ if and only if $\xi=0$ which means that solving $\psi \hat{u}=(2 \pi)^{-\frac{n}{2}}$ and then try to find the inverse Fourier transform of $\frac{1}{(2 \pi)^{\frac{n}{2}} \psi}$ is a problem.

Since $\mathcal{S}\left(\mathbb{R}^{n}\right) \subset H^{\psi, s}\left(\mathbb{R}^{n}\right) \subset \mathcal{S}^{\prime}\left(\mathbb{R}^{n}\right)$, under the additional condition $(1+\psi(\cdot))^{-s_{0}} \in$ $L^{1}\left(\mathbb{R}^{n}\right)$, we first deduce that now $\psi(\cdot) \hat{u}=(2 \pi)^{-\frac{n}{2}}$ is an equation in $L^{\infty}\left(\mathbb{R}^{n}\right)$ or 
even in $L_{l o c}^{1}\left(\mathbb{R}^{n}\right)$ and the question arises whether we can conclude that $\frac{1}{\psi(\cdot)}$ give rise to an element in $\mathcal{S}^{\prime}\left(\mathbb{R}^{n}\right)$ such that its inverse Fourier transform can be used to define a "nice" fundamental solution for $\psi(D)$.

Example 4.4.3. For $0<\alpha<2$ the function $\xi \mapsto|\xi|^{\alpha}$ is a continuous negative definite function which for $0<\alpha \leq 1$ it is not differentiable and for $1<\alpha<2$ it is a $C^{1}$-function but not $C^{2}$. However it is a homogeneous function as $|\xi|^{-\alpha}$ is on $\mathbb{R}^{n} \backslash\{0\}$, and homogeneous functions give rise to homogeneous distributions which do belong to $\mathcal{S}^{\prime}\left(\mathbb{R}^{n}\right)$. Thus $F^{-1}\left(\frac{1}{|\cdot|^{\alpha}}\right), 0<\alpha<2$, is a well defined element in $\mathcal{S}^{\prime}\left(\mathbb{R}^{n}\right)$. Indeed $F^{-1}\left(\frac{1}{|\cdot| \alpha}\right)(x)=c_{n, \alpha}|x|^{-n+\alpha}$ and we may try to use $E_{n, \alpha}=\tilde{c}_{n, \alpha}|\cdot|^{-n+\alpha} \in \mathcal{S}^{\prime}\left(\mathbb{R}^{n}\right)$ as a fundamental solution to $-(-\Delta)^{\frac{\alpha}{2}}$. In particular we may consider $u:=E_{n, \alpha} * g$ for certain classes of distributions $g$ and try to prove that $\psi(D) u=g$. Note that the restriction $0<\alpha<2$ is not really necessary, $\alpha>0$ will not change the argument. However properties of $E_{n, \alpha}$ may change. In particular, since

$$
\int_{B_{R}(0)} E_{n, \alpha}(x) d x=c \int_{0}^{R} \rho^{-n+\alpha} \rho^{n-1} d \rho=c \int_{0}^{R} \rho^{\alpha-1} d \rho
$$

we may allow $\alpha>0$.

Let us return to the general case. The symbols we are interested in most are real-valued continuous negative definite functions and they are even, i.e. $\psi(\xi)=$ $\psi(-\xi)$. For even functions as well as for even distributions we have $F^{2} u=u$.

Theorem 4.4.4. Let $\psi: \mathbb{R}^{n} \rightarrow \mathbb{R}$ be a continuous negative definite function such that for some $s_{0}>0$ we have $(1+\psi(\cdot))^{-\frac{s_{0}}{2}} \in L^{2}\left(\mathbb{R}^{n}\right)$ and suppose $\left.\frac{1}{\psi}\right|_{B_{1}(0)} \in$ $L^{2}\left(B_{1}(0)\right)$ and $\psi(\xi)=0$ if and only if $\xi=0$. Then the distribution $E \in \mathcal{S}^{\prime}\left(\mathbb{R}^{n}\right)$ defined by $\hat{E}=\frac{1}{\psi}$ belongs to $H^{\psi,-\frac{s_{0}}{2}}\left(\mathbb{R}^{n}\right)$. 
Proof. For $v \in \mathcal{S}\left(\mathbb{R}^{n}\right)$ and $c:=\sup _{\xi \in B_{1}^{c}(0)} \frac{1}{\psi(\xi)}$ it follows that

$$
\begin{aligned}
\left|\left\langle\frac{1}{\psi}, \hat{v}\right\rangle\right| & \leq\left|\int_{B_{1}(0)} \frac{1}{\psi} \hat{v} d \xi\right|+\left|\int_{B_{1}^{c}(0)} \frac{1}{\psi} \hat{v} d \xi\right| \\
& \leq\left\|\frac{1}{\psi}\right\|_{L^{2}\left(B_{1}(0)\right)}\|\hat{v}\|_{L^{2}\left(B_{1}(0)\right)}+c \int_{B_{1}^{c}(0)}|\hat{v}(\xi)| d \xi \\
& \leq\left\|\frac{1}{\psi}\right\|_{L^{2}\left(B_{1}(0)\right)}\|\hat{v}\|_{L^{2}\left(\mathbb{R}^{n}\right)}+c \int_{B_{1}^{c}(0)}(1+\psi(\xi))^{-\frac{s_{0}}{2}}(1+\psi(\xi))^{\frac{s_{0}}{2}}|\hat{v}(\xi)| d \xi \\
& \leq\left\|\frac{1}{\psi}\right\|_{L^{2}\left(B_{1}(0)\right)}\|v\|_{L^{2}\left(\mathbb{R}^{n}\right)}+c\left\|(1+\psi(\cdot))^{-\frac{s_{0}}{2}}\right\|_{L^{2}}\|v\|_{H^{\psi}, s_{0}} \\
& \leq\left(\left\|\frac{1}{\psi}\right\|_{L^{2}\left(B_{1}(0)\right)}+c\left\|(1+\psi(\cdot))^{-\frac{s_{0}}{2}}\right\|_{L^{2}}\right)\|v\|_{H^{\psi, s_{0}}},
\end{aligned}
$$

which implies that $\frac{1}{\psi} \in H^{\psi,-s_{0}}\left(\mathbb{R}^{n}\right)$, hence $\frac{1}{\psi} \in \mathcal{S}^{\prime}\left(\mathbb{R}^{n}\right)$ and in $\mathcal{S}^{\prime}\left(\mathbb{R}^{n}\right)$ we have for $E:=F^{-1}\left(\frac{1}{\psi}\right)$ that $\hat{E}=\frac{1}{\psi}$.

Remark 4.4.5. Note that $\left.\frac{1}{\psi}\right|_{B_{1}(0)} \in L^{2}\left(B_{1}(0)\right)$ implies that $\left.\frac{1}{\psi}\right|_{B_{1}(0)} \in L^{1}\left(B_{1}(0)\right)$, hence $\frac{1}{\psi} \in L_{\text {loc }}^{1}\left(\mathbb{R}^{n}\right)$.

Now we can give

Definition 4.4.6. Let $\psi: \mathbb{R}^{n} \rightarrow \mathbb{R}$ be a continuous negative definite function such that $\psi(\xi)=0$ if and only if $\xi=0$, for some $s_{0} \in \mathbb{R}$ we have $(1+\psi(\cdot))^{-\frac{s_{0}}{2}} \in$ $L^{2}\left(\mathbb{R}^{n}\right)$, and $\left.\frac{1}{\psi}\right|_{B_{1}(0)} \in L_{l o c}^{2}\left(B_{1}(0)\right)$. Then we call the unique tempered distribution $E \in H^{\psi,-s_{0}}\left(\mathbb{R}^{n}\right)$ satisfying $\hat{E}=\frac{1}{\psi}$ the $H^{\psi, s_{0}}$-fundamental solution to $\psi(D)$.

Remark 4.4.7. Of course we obtain trivially a fundamental solution to $\psi(D)$ in $H^{\psi, s_{0}}\left(\mathbb{R}^{n}\right)$ if all conditions of Definition 4.4 .6 are satisfied but $\psi(\xi)=0$ if and only if $\xi=0$ is replaced by $\inf _{\xi \in \mathbb{R}^{n}} \psi(\xi) \geq \kappa_{0}>0$. In this case $E \in \mathcal{S}^{\prime}\left(\mathbb{R}^{n}\right)$ with $\hat{E}=\frac{1}{(2 \pi)^{\frac{n}{2}} \psi(\cdot)}$ belongs obviously to $H^{\psi,-s_{0}}\left(\mathbb{R}^{n}\right)$ since $\frac{1}{(2 \pi)^{\frac{n}{2}} \psi} \leq \frac{1}{(2 \pi)^{\frac{n}{2}} \kappa_{0}}$. Moreover, if $\psi$ is replaced by a function $k: \mathbb{R}^{n} \rightarrow \mathbb{R}$ which is continuous, having the only zero at 0 and is at most of polynomial growth, then the condition $(1+k(\cdot))^{-\frac{s_{0}}{2}} \in$ $L^{2}\left(\mathbb{R}^{n}\right)$ for some $s_{0} \in \mathbb{R}$ implies the existence of a fundamental solution $E$ in $H^{k,-s_{0}}$ with $\hat{E}=\frac{1}{(2 \pi)^{\frac{n}{2} k}}$. Again the case $k(\xi) \geq \kappa_{0}>0$ for all $\xi \in \mathbb{R}$ is trivial.

Remark 4.4.8. It is not clear whether the condition $\frac{1}{\psi} \in L_{\text {loc }}^{1}\left(\mathbb{R}^{n}\right)$ alone is sufficient to deduce that $E:=F^{-1}\left(\frac{1}{\psi}\right) \in \mathcal{S}^{\prime}\left(\mathbb{R}^{n}\right)$. However, $\left.\frac{1}{\psi}\right|_{B_{1}(0)} \in L^{p}\left(B_{1}(0)\right)$, 
$1<p<\infty$, allows the estimate

$$
\left|\int_{B_{1}(0)} \frac{1}{\psi} \hat{v} d x\right| \leq\left\|\frac{1}{\psi}\right\|_{L^{p}\left(B_{1}(0)\right)}\|\hat{v}\|_{L^{p^{\prime}\left(\mathbb{R}^{n}\right)}}
$$

with $\frac{1}{p}+\frac{1}{p^{\prime}}=1$. For $1<p \leq 2$ we have further the estimate

$$
\|\hat{v}\|_{L^{p^{\prime}}} \leq(2 \pi)^{-\frac{(2-p) n}{2 p}}\|v\|_{L^{p}}
$$

see Theorem 3.2.20 in [20]. Now one may use the theory of $\psi$-Bessel potential spaces as introduced in [6] to obtain the result of Theorem 4.4 .4 under the condition $\left.\frac{1}{\psi}\right|_{B_{1}(0)} \in L^{p}\left(B_{1}(0)\right)$ with $H^{\psi, s}\left(\mathbb{R}^{n}\right)$ replaced by $H_{p}^{\psi, s}\left(\mathbb{R}^{n}\right)$. We refer to the forthcoming paper, [8], for details.

Since under the assumption of Theorem 4.4 .4 we know that $\psi(D)$ admits a fundamental solution $E$ in $H^{\psi,-s_{0}}\left(\mathbb{R}^{n}\right) \subset \mathcal{S}^{\prime}\left(\mathbb{R}^{n}\right)$, it follows that for $g \in \mathcal{S}(\mathbb{R})$ a solution to $\psi(D) u=g$ is given by $u:=E * g$. In order to increase the class of functions $g$ as right hand sides we prove first

Proposition 4.4.9. For $v \in H^{\psi,-s}\left(\mathbb{R}^{n}\right)$ and $u \in H^{\psi, s+t}\left(\mathbb{R}^{n}\right)$ the convolution $v * u$ is defined and $(1+\psi(D))^{\frac{t}{2}}(v * u)$ belongs to $C_{b}\left(\mathbb{R}^{n}\right)$.

Proof. For $u, v \in \mathcal{S}\left(\mathbb{R}^{n}\right)$ we find by Young's inequality

$$
\begin{aligned}
\left\|(1+\psi(D))^{\frac{t}{2}}(v * u)\right\|_{\infty} & =\left\|(1+\psi(D))^{-\frac{s}{2}} v *(1+\psi(D))^{\frac{t+s}{2}} u\right\|_{\infty} \\
& \leq\left\|(1+\psi(D))^{-\frac{s}{2}} v\right\|_{L^{2}}\left\|(1+\psi(D))^{\frac{t+s}{2}} u\right\|_{L^{2}} \\
& =\|v\|_{\psi,-s}\|u\|_{\psi, s+t} .
\end{aligned}
$$

Since $\mathcal{S}\left(\mathbb{R}^{n}\right)$ is dense in $H^{\psi,-s}\left(\mathbb{R}^{n}\right)$ we first derive for all $u \in \mathcal{S}\left(\mathbb{R}^{n}\right)$ the estimate $\left\|(1+\psi(D))^{\frac{t}{2}}(v * u)\right\|_{\infty} \leq\|v\|_{\psi,-s}\|u\|_{\psi, s+t}$ for $v \in H^{\psi,-s}\left(\mathbb{R}^{n}\right)$ and now we use the density of $\mathcal{S}\left(\mathbb{R}^{n}\right)$ in $H^{\psi, s+t}\left(\mathbb{R}^{n}\right)$ to get this estimate for all $v \in H^{\psi,-s}\left(\mathbb{R}^{n}\right)$ and $u \in H^{\psi, s+t}\left(\mathbb{R}^{n}\right)$.

Corollary 4.4.10. If $g \in H^{\psi, s_{0}+t}\left(\mathbb{R}^{n}\right)$ with $\psi$ as in Theorem 4.4.4 then $u=$ $E * g \in H^{\psi, t}\left(\mathbb{R}^{n}\right)$ solves the equation $\psi(D) u=g$ which we may interpret as an equation in $H^{\psi, t-2}\left(\mathbb{R}^{n}\right)$. In particular, for $g \in H^{\psi, s_{0}+2}\left(\mathbb{R}^{n}\right)$ this equation admits a solution $u \in H^{\psi, 2}\left(\mathbb{R}^{n}\right)$ and $\psi(D) u=g$ holds in $L^{2}\left(\mathbb{R}^{n}\right)$. 
We note that the result of Corollary 4.4 .10 is not the result we shall expect in light of the results known for the Laplace operator. We want to prove that $g \in H^{\psi, t}\left(\mathbb{R}^{n}\right)$ implies $u \in H^{\psi, t+2}\left(\mathbb{R}^{n}\right)$ and will return to this question later on. Moreover, we may obtain better results than those in Proposition 4.4.9 using the $\psi$-Bessel potential spaces in an $L^{p}$ - setting. We refer to the forthcoming paper [8].

The Lévy Khinchin formula allows to represent $A^{\psi}=-\psi(D)$, the generator of the semigroup $\left(T_{t}^{\psi}\right)_{t \geq 0}$, as an integro-differential operator. Suppose $\psi$ is realvalued and has no polynomial part. In this case $\psi(D) \operatorname{admits}$ on $\mathcal{S}\left(\mathbb{R}^{n}\right)$ a representation

$$
\psi(D) u(x)=\int_{\mathbb{R}^{n}}(u(x+y)-u(x)) \tilde{\nu}(d y)
$$

with $\tilde{\nu}$ being (the modified) Lévy measure corresponding to $\psi$. From (4.14) it is clear that for $u \in C_{0}^{\infty}\left(\mathbb{R}^{n}\right)$ the function $\psi(D) u$ will in general have no compact support. This causes problems to extend $\psi(D)$ to $\mathscr{D}^{\prime}\left(\mathbb{R}^{n}\right)$. The defining formula would be

$$
\langle\psi(D) u, \varphi\rangle=\langle u, \psi(D) \varphi\rangle, \varphi \in C_{0}^{\infty}\left(\mathbb{R}^{n}\right)
$$

where we have taken into account that $\psi(D)$ is symmetric, for example on $\mathcal{S}\left(\mathbb{R}^{n}\right)$. In order that (4.15) makes sense for $u \in \mathscr{D}^{\prime}\left(\mathbb{R}^{n}\right)$ we need to have $\psi(D) \varphi \in$ $C_{0}^{\infty}\left(\mathbb{R}^{n}\right)$, which however does not hold in general. Thus, switching from the pseudo-differential operator representation of $\psi(D)$ to the Lévy operator or von Waldenfels operator representation does not change the type of problem we face when trying to define a fundamental solution to $\psi(D)$. 


\section{Chapter 5}

\section{Transient Semigroups, Potential Kernels and Fundamental Solutions}

For a continuous negative definite function $\psi: \mathbb{R}^{n} \rightarrow \mathbb{R}$ such that $(1+\psi)^{-\frac{s_{0}}{2}} \in$ $L^{2}\left(\mathbb{R}^{n}\right)$ for some $s_{0} \in \mathbb{R}$ and for which $\frac{1}{\psi} \in L_{\text {loc }}^{2}\left(\mathbb{R}^{n}\right)$ we can find a $H^{\psi, s_{0}}$ fundamental solution $E$ which admits to solve $\psi(D) u=g$ for $g \in H^{\psi, s_{0}+2}\left(\mathbb{R}^{n}\right)$ by $E * g$. The regularity assumption on $g$ is rather strong, but we shall have in mind that in the $H^{\psi, s_{0}}-H^{\psi,-s_{0}}$ context our result is also a uniqueness result. Since $E \in \mathcal{S}^{\prime}\left(\mathbb{R}^{n}\right)$ we can form further convolutions, not just $E * g$ for $g \in H^{\psi, s_{0}}\left(\mathbb{R}^{n}\right)$. For example $g \in \mathcal{E}^{\prime}\left(\mathbb{R}^{n}\right)$ is possible since the convolution of any distribution $u \in \mathscr{D}^{\prime}\left(\mathbb{R}^{n}\right)$ with a distribution of compact support is defined. However, since $u \in \mathscr{D}^{\prime}\left(\mathbb{R}^{n}\right)$ need not have a Fourier transform the convolution thereom does not apply in this situation and it is the need of the convolution theorem to hold that requires a type of duality in the regularity of $u$ and $g$ when forming $u * g$. We can form $u * g$ and apply the convolution theorem for $u \in \mathcal{S}^{\prime}\left(\mathbb{R}^{n}\right)$ and $g \in \mathcal{S}\left(\mathbb{R}^{n}\right)$, or as we have proved when working with the pairing $H^{\psi, s_{0}}$ and $H^{\psi,-s_{0}}$. A natural question is whether we can obtain more mapping properties (regularity) for $g \mapsto E * g, \hat{E}=\frac{1}{\psi}$, when $g$ belongs to larger classes than 
$H^{\psi, s_{0}}\left(\mathbb{R}^{n}\right)$ or $H^{\psi, s_{0}+t}\left(\mathbb{R}^{n}\right)$. The aim of this chapter is to identify in certain situations $E$ with the kernel of the (abstract) potential operator corresponding to the Dirichlet form associated with $\psi$. This is possible in the context of the extended Dirichlet space corresponding to $\psi$ which we will introduce now. Note that when only dealing with the extended Dirichlet space associated with $\psi$ the condition $\frac{1}{\psi} \in L_{l o c}^{1}\left(\mathbb{R}^{n}\right)$ is sufficient, but once we want to identify $F^{-1}\left(\frac{1}{\psi}\right)$ with a fundamental solution of $\psi(D)$, our results require $\frac{1}{\psi} \in L_{l o c}^{2}\left(\mathbb{R}^{n}\right)$.

\subsection{Translation Invariant Extended Dirichlet Spaces}

Let $\left(T_{t}\right)_{t \geq 0}$ be a symmetric $L^{2}$ sub-Markovian semigroup on $L^{2}\left(\mathbb{R}^{n}\right)$.

We define the operator

$$
S_{t} u:=\int_{0}^{t} T_{s} u d s, \quad t>0
$$

where the integral is defined as the Bochner integral in $L^{2}\left(\mathbb{R}^{n}\right)$. Since we have a symmetric strongly continuous contraction semigroup in $L^{2}$ the semigroup $\left(T_{t}\right)_{t \geq 0}$ extends from $L^{2}\left(\mathbb{R}^{n}\right) \cap L^{p}\left(\mathbb{R}^{n}\right)$, to $L^{p}\left(\mathbb{R}^{n}\right)$ as a sub-Markovian semigroup, $1 \leq p<\infty$, and we have the following estimate

$$
\left\|S_{t} u\right\|_{L^{p}} \leq t\|u\|_{L^{p}}
$$

In addition, $S_{t}$ is positivity preserving. We take the following definition from [11].

Definition 5.1.1. The potential operator $G$ associated with $\left(T_{t}\right)_{t \geq 0}$ is defined for $u \in L^{1}\left(\mathbb{R}^{n}\right), u \geq 0$ a.e., by

$$
G u(x):=\lim _{N \rightarrow \infty} S_{N} u(x)=\sup _{N \in \mathbb{N}} S_{N} u(x) .
$$


The limit in (5.1) exists a.e. but it may take the value $+\infty$. Moreover, since

$$
\begin{aligned}
\sup _{N \in \mathbb{N}} S_{N} u(x) & =\sup _{N \in \mathbb{N}} \sup _{\lambda>0} \int_{0}^{N} e^{-\lambda t} T_{t} u(x) d t \\
& =\sup _{\lambda>0} \sup _{N \in \mathbb{N}} \int_{0}^{N} e^{-\lambda t} T_{t} u(x) d t=\sup _{\lambda>0} R_{\lambda} u(x),
\end{aligned}
$$

we have

$$
\lim _{N \rightarrow \infty} S_{N} u(x)=\lim _{\lambda \rightarrow 0} R_{\lambda} u(x) \text { a.e. }
$$

Definition 5.1.2. If $G u<\infty$ a.e. for all $u \in L^{1}\left(\mathbb{R}^{n}\right), u \geq 0$ a.e., then we call $\left(T_{t}\right)_{t \geq 0}$ transient. In the case that for all $u \in L^{1}\left(\mathbb{R}^{n}\right), u \geq 0$ a.e., we have $G u(x) \in\{0, \infty\}$ for almost all $x \in \mathbb{R}^{n}$ we call $\left(T_{t}\right)_{t \geq 0}$ recurrent.

Any strongly continuous transient sub-Markovian semigroup $\left(T_{t}\right)_{t \geq 0}$ admits a strictly positive bounded integrable function $g$ such that $\int g \cdot G g d x \leq 1$, compare with [11]. We call $g$ a reference function of the transient semigroup $\left(T_{t}\right)_{t \geq 0}$. To construct $g$ we take a strictly positive bounded measurable function $f \in L^{1}\left(\mathbb{R}^{n}\right)$ with $\int f d x=1$ and let $g=f /(G f \vee 1)$. The function $g$ is dominated by $f$ and strictly positive a.e. and we have $\int g \cdot G g d x \leq \int f \cdot G g d x \leq \int G f \cdot(f / G f) d x=$ $\int f d x=1$. Therefore we have from [11]

Theorem 5.1.3. Let $\left(T_{t}\right)_{t \geq 0}$ be a symmetric $L^{2}$ sub-Markovian semigroup and $(\mathcal{E}, D(\mathcal{E}))$ the corresponding Dirichlet form. The semigroup is transient if and only if there exists a bounded function $g \in L^{1}\left(\mathbb{R}^{n}\right)$ which is strictly positive and satisfies for all $u \in D(\mathcal{E})$

$$
\int|u| g d x \leq \mathcal{E}^{\frac{1}{2}}(u, u)
$$

The transience of a Dirichlet form depends only on $\left(T_{t}\right)_{t \geq 0}$, which is the symmetric $L^{2}$ sub-Markovian semigroup associated with the symmetric Dirichlet form $\left(\mathcal{E}^{\text {sym }}, D(\mathcal{E})\right)$. Therefore we will study in more detail the symmetric $L^{2}$ sub-Markovian semigroups and their corresponding Dirichlet forms, leading to extended Dirichlet spaces.

Definition 5.1.4. Let $(\mathcal{E}, D(\mathcal{E}))$ be a symmetric Dirichlet form on $L^{2}\left(\mathbb{R}^{n}\right)$, i.e. $D(\mathcal{E}) \subset L^{2}\left(\mathbb{R}^{n}\right)$. The extended Dirichlet space $\mathscr{F}_{e}$ associated with $(\mathcal{E}, D(\mathcal{E}))$ is 
the family of all measurable functions $u: \mathbb{R}^{n} \rightarrow \mathbb{R},|u|<\infty$ a.e., such that there exists a sequence $\left(u_{k}\right)_{k \in \mathbb{N}}, u_{k} \in D(\mathcal{E})$, which converges almost everywhere to $u$ and which is a Cauchy sequence with respect to $\mathcal{E}$, i.e. $\mathcal{E}\left(u_{k}-u_{l}, u_{k}-u_{l}\right) \rightarrow 0$ as $k, l \rightarrow \infty$.

Note that it follows from the definition that $u \in \mathscr{F}_{e}$ is an element in $L_{l o c}^{1}\left(\mathbb{R}^{n}\right)$. However, in general $\mathscr{F}_{e}$ will not belong to $L^{2}\left(\mathbb{R}^{n}\right)$. We will now look at some basic properties of the extended Dirichlet space in the transient case. The following is taken from [11], Theorem 1.5.3.

Theorem 5.1.5. A pair $\left(\mathscr{F}_{e}, \mathcal{E}\right)$ is the extended Dirichlet space of a transient Dirichlet spacec relative to $L^{2}\left(\mathbb{R}^{n}\right)$ if and only if the following conditions are satisfied:

(i) $\mathscr{F}_{e}$ is a real Hilbert space with inner product $\mathcal{E}$;

(ii) there exists a bounded function $g$ strictly positive such that $\mathscr{F}_{e} \subset L^{1}\left(\mathbb{R}^{n}\right)$ and

$$
\int|u| g d x \leq \mathcal{E}^{\frac{1}{2}}(u, u)
$$

(iii) $\mathscr{F}_{e} \cap L^{2}\left(\mathbb{R}^{n}\right)$ is dense both in $L^{2}\left(\mathbb{R}^{n}\right)$ and in $\left(\mathscr{F}_{e}, \mathcal{E}\right)$;

(iv) every normal contraction operates on $\left(\mathscr{F}_{e}, \mathcal{E}\right)$, i.e. if $u \in \mathscr{F}_{e}$, and $v$ is a normal contraction of $u$ then $v \in \mathscr{F}_{e}$ and $\mathcal{E}(v, v) \leq \mathcal{E}(u, u)$.

In this case, $\left(\mathscr{F}_{e} \cap L^{2}\left(\mathbb{R}^{n}\right), \mathcal{E}\right)$ is a Dirichlet space relative to $L^{2}\left(\mathbb{R}^{n}\right)$ and $\left(\mathscr{F}_{e}, \mathcal{E}\right)$ is its extended Dirichlet space.

Definition 5.1.6. Let $\left(T_{t}\right)_{t \geq 0}$ be a symmetric sub-Markovian semigroup on $L^{2}\left(\mathbb{R}^{n}\right)$ with generator $(A, D(A))$, resolvent $\left(R_{\lambda}\right)_{\lambda>0}$ and potential operator $(G, D(G))$ where

$$
D(G):=\left\{u \in L^{1}\left(\mathbb{R}^{n}\right) \mid u \geq 0 \text { a.e. and } \lim _{N \rightarrow \infty} \int_{0}^{N} T_{t} u d t \text { exists in } L^{1}\left(\mathbb{R}^{n}\right)\right\} .
$$

The abstract potential operator or the resolvent at zero $\left(R_{0}, D\left(R_{0}\right)\right)$ associated with $\left(T_{t}\right)_{t \geq 0}$ is the operator $R_{0}$ defined on

$$
D\left(R_{0}\right):=\left\{u \in L^{2}\left(\mathbb{R}^{n}\right) \mid \lim _{\lambda \rightarrow 0} R_{\lambda} u \text { exists in } L^{2}\left(\mathbb{R}^{n}\right)\right\}
$$


and we set

$$
R_{0} u=\lim _{\lambda \rightarrow 0} R_{\lambda} u, u \in D\left(R_{0}\right)
$$

We want to emphasize that $D(G)$ is not a vector space while $D\left(R_{0}\right)$ is.

The following result is taken from [21].

Theorem 5.1.7. Let $\left(T_{t}\right)_{t \geq 0}$ be a symmetric transient sub-Markovian semigroup on $L^{2}\left(\mathbb{R}^{n}\right)$ with generator $(A, D(A))$. In this case the corresponding potential operator and the abstract potential operator coincide as $L^{2}$-operators and we have $A=-G^{-1}$ as well as $G=-A^{-1}$ where $A u \in D\left(R_{0}\right)$, given that $u \in D(A)$.

Let $\left(\mu_{t}\right)_{t \geq 0}$ be a symmetric convolution semigroup on $\mathbb{R}^{n}$ with associated negative definite function $\psi: \mathbb{R}^{n} \rightarrow \mathbb{R}$. Since, we assume $\psi(\xi)=0$ if and only if $\xi=0$, it follows that all the measures $\mu_{t}$ are probability measures. The following results are taken from [21], Theorem 3.5.51.

Theorem 5.1.8. Let $\left(\mu_{t}\right)_{t \geq 0}$ be a symmetric convolution semigroup of probability measures on $\mathbb{R}^{n}$ with corresponding continuous negative definite function $\psi$ : $\mathbb{R}^{n} \rightarrow \mathbb{R}$ and symmetric Dirichlet form $\left(\mathcal{E}^{\psi}, D\left(\mathcal{E}^{\psi}\right)\right)=\left(\mathcal{E}^{\psi}, H^{\psi, 1}\left(\mathbb{R}^{n}\right)\right)$, where

$$
\mathcal{E}^{\psi}(u, v)=\int_{\mathbb{R}^{n}} \psi(\xi) \hat{u}(\xi) \overline{\hat{v}(\xi)} d \xi
$$

Denote the operator semigroup $\left(T_{t}^{\psi}\right)_{\geq 0}$ with $T_{t}^{\psi}$, i.e.

$$
T_{t}^{\psi} u(x)=\int_{\mathbb{R}^{n}} u(x-y) \mu_{t}(d y)
$$

The following statements are equivalent

(i) $\left(T_{t}^{\psi}\right)_{t \geq 0}$ is transient;

(ii) for every compact set $K \subset \mathbb{R}^{n}$ we have

$$
\kappa(K):=\int_{0}^{\infty} \mu_{t}(K) d t<\infty
$$


(iii) for all $u \in C_{0}\left(\mathbb{R}^{n}\right), u \geq 0$, it follows that

$$
\int_{0}^{\infty}\left(T_{t}^{\psi} u, u\right)_{0} d t<\infty
$$

(iv) $\frac{1}{\psi} \in L_{l o c}^{1}\left(\mathbb{R}^{n}\right)$.

We will discuss the kernel defined in $(i i)$ in the next section. As a corollary to Theorem 5.1.7 we have

Corollary 5.1.9. Let $\left(T_{t}^{\psi}\right)_{t \geq 0}$ be a transient symmetric $L^{2}$ sub-Markovian semigroup associated with the convolution semigroup $\left(\mu_{t}\right)_{t \geq 0}$ and the corresponding negative definite function $\psi$. In this case the potential operator $G$ and the abstract operator $R_{0}$ are both defined as $L^{2}$-operators and coincide. Moreover $R_{0}$ is densely defined.

The following examples are taken from [20].

Example 5.1.10. Let $\psi: \mathbb{R}^{n} \rightarrow \mathbb{R}$ be a continuous negative definite function such that $\frac{1}{\psi} \in L_{\text {loc }}^{1}\left(\mathbb{R}^{n}\right)$. The symmetric Dirichlet space $(\mathcal{E}, D(\mathcal{E}))$ associated with $\psi$ is given by

$$
D(\mathcal{E})=H^{\psi, 1}\left(\mathbb{R}^{n}\right)
$$

and

$$
\mathcal{E}(u, v)=\int_{\mathbb{R}^{n}} \psi(\xi) \hat{u}(\xi) \overline{\hat{u}(\xi)} d \xi .
$$

The corresponding extended Dirichlet space is given by

$$
\mathcal{F}_{e}:=H_{e}^{\psi, 1}\left(\mathbb{R}^{n}\right):=\left\{u \in \mathcal{S}^{\prime} \mid u \in L_{l o c}^{1}\left(\mathbb{R}^{n}\right) \text { and } \hat{u} \psi^{\frac{1}{2}} \in L^{2}\left(\mathbb{R}^{n}\right)\right\}
$$

Note that $\hat{u} \psi^{\frac{1}{2}} \in L^{2}\left(\mathbb{R}^{n}\right)$ if and only if $\hat{u}$ belongs to the weighted $L^{2}$-space $L^{2}\left(\mathbb{R}^{n} ; \psi \lambda^{(n)}\right)$ with norm

$$
\|u\|_{H_{e}^{\psi, 1}}^{2}:=\|u\|_{L^{2}\left(\mathbb{R}^{n} ; \psi \lambda(n)\right)}^{2}=\int_{\mathbb{R}^{n}}|\hat{u}(\xi)|^{2} \psi(\xi) d \xi
$$

In general it is not known whether we can prove $H_{e}^{\psi, 1}\left(\mathbb{R}^{n}\right) \subset L^{p}\left(\mathbb{R}^{n}\right)$ for some $p \geq 1$, in fact we shall not expect such an inclusion in the general case. 
Example 5.1.11. Consider $\psi(\xi)=|\xi|^{\alpha}, 0<\alpha<2$, with corresponding operator semigroup

$$
T_{t}^{\psi} u(x)=T_{t}^{(\alpha)} u(x)=(2 \pi)^{-\frac{n}{2}} \int_{\mathbb{R}^{n}} e^{i x \xi} e^{-t|\xi|^{\alpha}} \hat{u}(\xi) d \xi
$$

Since

$$
\int_{B_{R}(0)} \frac{1}{|\xi|^{\alpha}} d \xi=c_{n} \int_{0}^{R} r^{n-1-\alpha} d r
$$

it follows that $\frac{1}{\psi} \in L_{\text {loc }}^{1}\left(\mathbb{R}^{n}\right)$ if and only if $\alpha<n$, i.e. $\left(T_{t}^{(\alpha)}\right)_{t \geq 0}$ is transient for all $n \geq 2$. For the potential operator $G^{(\alpha)}$ we find for $u \geq 0$ that

$$
\begin{aligned}
G^{(\alpha)} u(x) & =\int_{0}^{\infty} T_{t}^{(\alpha)} u(x) d t=(2 \pi)^{-\frac{n}{2}} \int_{0}^{\infty} \int_{\mathbb{R}^{n}} e^{i x \xi} e^{-t|\xi|^{\alpha}} \hat{u}(\xi) d \xi d t \\
& =(2 \pi)^{-\frac{n}{2}} \int_{\mathbb{R}^{n}} e^{i x \xi} \int_{0}^{\infty} e^{-t|\xi|^{\alpha}} d t \hat{u}(\xi) d \xi \\
& =(2 \pi)^{-\frac{n}{2}} \int_{\mathbb{R}^{n}} e^{i x \xi} \frac{1}{|\xi|^{\alpha}} \hat{u}(\xi) d \xi
\end{aligned}
$$

where we are able to interchange the integrals since

$$
\int_{0}^{\infty} \int_{\mathbb{R}^{n}}\left|e^{i x \xi} e^{-t|\xi|^{\alpha}} \hat{u}(\xi)\right| d \xi d t<\infty
$$

With the Riesz-kernel $R^{(\alpha)}(x)=c_{\alpha, n}|x|^{-n+\alpha}$ where

$$
c_{\alpha, n}=\frac{\Gamma\left(\frac{n-\alpha}{2}\right)}{2^{\alpha} \pi^{\frac{n}{2}} \Gamma\left(\frac{\alpha}{2}\right)},
$$

we have by the convolution theorem

$$
G^{(\alpha)}=R^{(\alpha)} * u=: R^{\alpha} u
$$

Note that $R^{(\alpha)}(x)$ is homogeneous of degree $-n+\alpha$. For $H_{e}^{\alpha}\left(\mathbb{R}^{n}\right)$, the extended Dirichlet space associated with $\left(T_{t}^{\alpha}\right)_{t \geq 0}$ in the transient case, we find

$$
H_{e}^{\alpha}\left(\mathbb{R}^{n}\right):=\left\{u \in L_{l o c}^{1}\left(\mathbb{R}^{n}\right) \mid u=R^{\frac{\alpha}{2}} g \text { for some } g \in L^{2}\left(\mathbb{R}^{n}\right)\right\} \text {. }
$$

In general, we know that $H_{e}^{\alpha}\left(\mathbb{R}^{n}\right)$ is a subspace of $L_{l o c}^{1}\left(\mathbb{R}^{n}\right)$. However, the homegenity of the kernel $R^{\alpha}$ also allows us to prove a variant of Sobolev's embedding theorem to find $H_{e}^{\alpha}\left(\mathbb{R}^{n}\right) \subset L^{p}\left(\mathbb{R}^{n}\right), p=\frac{2 n}{n-2 \alpha}$. We state Sobolev's inequality as given in [37]. 
Theorem 5.1.12. Let $1<q<p<\infty, 0<\alpha<n$ and $\frac{1}{p}=\frac{1}{q}-\frac{\alpha}{n}>0$. Then for all $u \in \mathcal{S}\left(\mathbb{R}^{n}\right)$ we have

$$
\left\|R^{\alpha} u\right\|_{L^{p}} \leq c_{0}\|u\|_{L^{q}}
$$

The proof of this theorem can be found in both [31] and [37]. The following results along with the proof of Corollary 5.1.14 is taken from [21].

Corollary 5.1.13. For $n \geq 2$ and $0<\alpha<2$ we have $H_{e}^{\frac{\alpha}{2}} \hookrightarrow L^{p}\left(\mathbb{R}^{n}\right)$ and the estimate

$$
\|u\|_{L^{p}}^{2} \leq c \mathcal{E}(u, u)
$$

holds for all $u \in H_{e}^{\frac{\alpha}{2}}\left(\mathbb{R}^{n}\right)$.

Corollary 5.1.14. Let $\psi: \mathbb{R}^{n} \rightarrow \mathbb{R}$ be a continuous negative definite function such that for some $c_{0}>0$ and $0<\alpha<2$ we have for all $\xi \in \mathbb{R}^{n}$

$$
c_{0}|\xi|^{\alpha} \leq \psi(\xi)
$$

If $n \geq 2$ then the semigroup $\left(T_{t}^{\psi}\right)_{t \geq 0}$ is transient and the extended Dirichlet space $H_{e}^{\psi, 1}\left(\mathbb{R}^{n}\right)$ is continuously embedded into $H_{e}^{\alpha}\left(\mathbb{R}^{n}\right)$, hence into $L^{p}\left(\mathbb{R}^{n}\right), p=\frac{2 n}{n-\alpha}$.

Proof. We observe that

$$
\int_{B_{R}(0)} \frac{1}{\psi(\xi)} \leq \frac{1}{c_{0}} \int_{B_{R}(0)} \frac{1}{|\xi|^{2 \alpha}} d \xi<\infty
$$

Moreover, we have

$$
\|u\|_{L^{p}}^{2} \leq c \int_{\mathbb{R}^{n}}|\xi|^{2 \alpha}|\hat{u}(\xi)|^{2} d \xi \leq \tilde{c} \int_{\mathbb{R}^{n}} \psi(\xi)|\hat{u}(\xi)|^{2} d \xi
$$

i.e.

$$
\|u\|_{L^{p}} \leq \tilde{c}\left(\mathcal{E}^{\psi}(u, u)\right)^{\frac{1}{2}}
$$

where $\mathcal{E}^{\psi}(u, v)=\int_{\mathbb{R}^{n}} \psi(\xi) \hat{u}(\xi) \overline{\hat{v}(\xi)} d \xi$ is the scalar product in the extended Dirichlet space $H_{(e)}^{\psi, 1}\left(\mathbb{R}^{n}\right)$. 


\subsection{Transient Convolution Semigroups}

In this section we mainly follow [2]. We have defined the potential operator for transient $L^{2}$ sub-Markovian semigroups. For a convolution semigroup $\left(\mu_{t}\right)_{t>0}$ we can introduce the measure

$$
\kappa=\int_{0}^{\infty} \mu_{t} d t
$$

which is known as the potential kernel for $\left(\mu_{t}\right)_{t>0}$ where the above integral is understood in terms of a convolution operator. If this measure exists, then the convolution semigroup $\left(\mu_{t}\right)_{t>0}$ is said to be transient. If $C_{0}\left(\mathbb{R}^{n}\right)$ is contained in the domain of the potential operator, then $\left(\mu_{t}\right)_{t>0}$ is said to be integrable, and it is shown that this is the case if and only if $\left(\mu_{t}\right)_{t>0}$ is transient and the potential kernel $\kappa$ tends to zero at infinity. We want to relate $\kappa$ to the resolvent. Given that $R_{\lambda}$ is a translation invariant operator, then it is a convolution operator and therefore we may assume that the operator $R_{\lambda}, \lambda>0$, admits the representation

$$
R_{\lambda} u=\rho_{\lambda} * u
$$

with

$$
\rho_{\lambda}=\int_{0}^{\infty} e^{-\lambda t} \mu_{t} d t
$$

where for all $u \in C_{0}\left(\mathbb{R}^{n}\right), u \geq 0$,

$$
\begin{aligned}
\left\langle\rho_{\lambda}, u\right\rangle & =\int_{\mathbb{R}^{n}} u(x) \rho_{\lambda}(d x) \\
& =\int_{0}^{\infty} e^{-\lambda t} \int_{\mathbb{R}^{n}} u(x) \mu_{t}(d x) d t \\
& =\int_{0}^{\infty} e^{-\lambda t}\left\langle\mu_{t}, u\right\rangle d t .
\end{aligned}
$$

Therefore, $\left(T_{t}\right)_{t \geq 0}$ is transient if and only if $\left(\mu_{t}\right)_{t \geq 0}$ is transient in the sense that

$$
\kappa(u):=\langle\kappa, u\rangle=\lim _{\lambda \rightarrow 0}\left\langle\rho_{\lambda}, u\right\rangle, \quad u \in C_{0}\left(\mathbb{R}^{n}\right)
$$

exists. We call $\kappa$ the potential kernel associated with $\left(\mu_{t}\right)_{t \geq 0}$. 
Given a continuous negative definite function on $\mathbb{R}^{n}$ associated with the convolution semigroup $\left(\mu_{t}\right)_{t>0}$, if $\psi(0)>0$ then the potential kernel $\kappa$ associated with $\left(\mu_{t}\right)_{t>0}$ is a bounded measure with total mass $\kappa\left(\mathbb{R}^{n}\right)=\frac{1}{\psi(0)}$ and its Fourier transform is given by

$$
\hat{\kappa}(\xi)=\frac{1}{\psi(\xi)} \text { for } \xi \in \mathbb{R}^{n}
$$

The potential kernel $\kappa$ for a transient convolution semigroup of probability measures on $\mathbb{R}^{n}$ is an unbounded measure, therefore the above equation has no meaning in this case. However, we are mainly interested in the case where $\psi(0)=0$. Therefore we need to generalise (5.3) to the case $\psi(0)=0$, using the theory of Fourier transformation of positive definite measures, i.e. distributions. We begin by defining a positive definite measure, taken from [2].

Definition 5.2.1. A measure $\mu$ on $\mathbb{R}^{n}$ is called positive definite if

$$
\langle\mu, g * \tilde{g}\rangle \geq 0 \text { for all } g \in C_{0}\left(\mathbb{R}^{n}\right)
$$

holds, where we denote by $\tilde{g}$ the function $\tilde{g}(x)=\overline{g(-x)}$.

Similarly, we have a positive definite distribution which we take from [20].

Definition 5.2.2. A distribution $u \in \mathscr{D}^{\prime}\left(\mathbb{R}^{n}\right)$ is called positive definite if

$$
\langle u, g * \tilde{g}\rangle \geq 0 \text { for all } g \in C_{0}^{\infty}\left(\mathbb{R}^{n}\right)
$$

holds, where we denote by $\tilde{g}$ the function $\tilde{g}(x)=\overline{g(-x)}$.

The Bochner-Schwartz Theorem, compare [34], Theorem 9.10, states

Theorem 5.2.3. A distribution $u \in \mathscr{D}^{\prime}\left(\mathbb{R}^{n}\right)$ is positive definite if and only if $u \in \mathcal{S}^{\prime}\left(\mathbb{R}^{n}\right)$ and $u$ is the Fourier transform of a positive measure of at most polynomial growth.

We find the following proposition in [2]. 
Proposition 5.2.4. Let $\psi$ be a continuous negative definite function on $\mathbb{R}^{n}$, and suppose that $\frac{1}{\psi}$ is integrable over some open, relatively compact neighbourhood of 0 . Then $\frac{1}{\psi}$ is locally integrable, and the measure $\frac{1}{\psi}$ is positive definite.

We can now link the notion to transcience and local integrability, see Theorem 13.15 in $[2]$.

Proposition 5.2.5. Let $\left(\mu_{t}\right)_{t>0}$ be a symmetric transient convolution semigroup on $\mathbb{R}^{n}$ with associated continuous negative definite function $\psi$ on $\mathbb{R}^{n}$. The function $\frac{1}{\psi}$ is locally integrable on $\mathbb{R}^{n}$.

Note that the above states that

$$
\left(\mu_{t}\right)_{t>0} \text { transient } \Longrightarrow \frac{1}{\psi} \text { locally integrable. }
$$

The converse also holds

$$
\frac{1}{\psi} \text { locally integrable } \Longrightarrow\left(\mu_{t}\right)_{t>0} \text { transient. }
$$

It is shown in [2] that the following lemma holds for the potential operator. However, it also holds for the potential kernel for the convolution semigroup $\left(\mu_{t}\right)_{t>0}$.

Lemma 5.2.6. The two conditions

(i) $C_{0}\left(\mathbb{R}^{n}\right) \subseteq D(G)$,

(ii) $C_{0}\left(\mathbb{R}^{n}\right) \subseteq D\left(R_{0}\right)$,

are equivalent.

Proof. (Compare with [2]) The zero resolvent is an extension of the potential kernel, therefore since $D(G) \subseteq D\left(R_{0}\right)$, it follows that $(i)$ implies $(i i)$. We can conclude this since if we find that the potential kernel is a subset of a continuous function with compact support, then this is also the case for the zero resolvent, 
due to the fact that the zero resolvent is a subset of the potential operator. Suppose, conversely that $C_{0}\left(\mathbb{R}^{n}\right) \subseteq D\left(R_{0}\right)$ and consider $u \in C_{0}^{+}\left(\mathbb{R}^{n}\right)$. The function $R_{0} u$ belongs to $C_{0}^{+}\left(\mathbb{R}^{n}\right)$, and for every $x \in \mathbb{R}^{n}$ we have

$$
R_{0} u(x)=\lim _{\lambda \rightarrow 0} R_{\lambda} u(x)=\int_{0}^{\infty} \mu_{t} u(x) d t
$$

by the monotone convergence theorem. Moreover we also have

$$
\lim _{s \rightarrow \infty} \int_{0}^{s} \mu_{t} u(x) d t=R_{0} u(x) \text { for } x \in \mathbb{R}^{n} .
$$

However this implies that

$$
\lim _{s \rightarrow \infty} \int_{0}^{s} \mu_{t} u d s=R_{0} u \text { uniformly on } \mathbb{R}^{n} .
$$

In addition, for a given $\varepsilon>0$ there exists a compact set $K \subseteq \mathbb{R}^{n}$ such that

$$
0 \leq R_{0} u(x) \leq \varepsilon \text { for all } x \notin K
$$

The convergence in (5.4) is increasing, and it follows that the convergence in (5.4) is uniform on $K$, i.e. there exists a $t_{0}>0$ such that

$$
\left|\int_{0}^{s} \mu_{t} u(x) d t-R_{0} u(x)\right| \leq \varepsilon \text { for } t \geq t_{0} \text { and } x \in K .
$$

This shows that

$$
\lim _{s \rightarrow \infty}\left\|\int_{0}^{s} \mu_{t} u d s-R_{0} u\right\|_{\infty}=0
$$

and it follows that $u \in D(G)$, which implies that $C_{0}\left(\mathbb{R}^{n}\right) \subseteq D(G)$.

Definition 5.2.7. A convolution semigroup $\left(\mu_{t}\right)_{t>0}$ on $\mathbb{R}^{n}$ is said to be integrable if the two conditions of Lemma 5.2.6 are satisfied.

Definition 5.2.8. A Borel measure $\mu$ is said to vanish at infinity if $\mu * f \in$ $C_{\infty}\left(\mathbb{R}^{n}\right)$ for all $f \in C_{0}\left(\mathbb{R}^{n}\right)$.

Now we can relate the transience of a convolution semigroup to its integrability which is taken from [2].

Proposition 5.2.9. A convolution semigroup $\left(\mu_{t}\right)_{t>0}$ on $\mathbb{R}^{n}$ is integrable if and only if $\left(\mu_{t}\right)_{t>0}$ is transient and the potential kernel $\kappa$ for $\left(\mu_{t}\right)_{>0}$ vanishes at infinity. 
We find the following theorem in [1] where we describe the kernel in more detail.

Theorem 5.2.10. Let $\left(\mu_{t}\right)_{t>0}$ be a convolution semigroup on $\mathbb{R}^{n}$ with associated continuous negative definite function $\psi$ on $\mathbb{R}^{n}$. If the function $\frac{1}{\psi}$ is locally integrable, then $\left(\mu_{t}\right)_{t>0}$ is integrable and the potential kernel $\kappa$ for $\left(\mu_{t}\right)_{t>0}$ is given by

$$
\kappa=F^{-1}\left(\frac{1}{\psi}\right) .
$$

The kernel (5.5) is the inverse Fourier transform of a positive definite measure. Therefore, due to Bochner-Schwartz Theorem, Theorem 5.2.3, we can conclude that the integral exists and is a positive definite tempered distribution.

Given previous results we may conclude that the following corollary holds.

Corollary 5.2.11. Let $\left(\mu_{t}\right)_{t>0}$ be a symmetric convolution semigroup on $\mathbb{R}^{n}$ with associated continuous negative definite function $\psi$ on $\mathbb{R}^{n}$. The following conditions are equivalent:

(i) $\left(\mu_{t}\right)_{t>0}$ is integrable;

(ii) $\left(\mu_{t}\right)_{t>0}$ is transient;

(iii) $\frac{1}{\psi}$ is locally integrable on $\mathbb{R}^{n}$.

Combining these results with our considerations on the $H^{\psi,-s_{0}}$-fundamental solutions for $\psi(D)$ we arrive at

Theorem 5.2.12. Let $\psi: \mathbb{R}^{n} \rightarrow \mathbb{R}$ be a continuous negative definite function such that $\frac{1}{\psi} \in L_{\text {loc }}^{2}\left(\mathbb{R}^{n}\right)$. The corresponding $L^{2}$ sub-Markovian semigroup $\left(T_{t}^{\psi}\right)_{t \geq 0}$ is transient and its potential kernel is given by $\kappa:=F^{-1}\left(\frac{1}{\psi}\right)$. In the case where $(1+\psi(\cdot))^{-\frac{s_{0}}{2}} \in L^{2}\left(\mathbb{R}^{n}\right)$ for some $s_{0}>0$ this kernel is the $H^{\psi,-s_{0}}$-fundamental solution of the operator $\psi(D)$.

Proof. The proof follows from Theorem 4.4.4 and Definition 4.4.6 along with the fact that the potential kernel defined by (5.5) in Theorem 5.2.10 gives a positive definite tempered distribution due to the Bochner-Schwartz Theorem. 
The main point of Theorem 5.2.12 is that it allows us to investigate the equation $\psi(D) u=f$ both in terms of the potential theory and the theory of distributions since the "potential theoretic inverse" of $\psi(D)$ admits on a large class of functions the same representation as the convolution operator induced by the fundamental solution, the first one is a pseudo-differential operator with symbol $\frac{1}{\psi}$, the second one is a convolution operator with kernel $F^{-1}\left(\frac{1}{\psi}\right)$.

\subsection{Fundamental Solutions as Potential Ker- nels}

Let $\psi: \mathbb{R}^{n} \rightarrow \mathbb{R}$ be a continuous negative definite function with corresponding convolution semigroup $\left(\mu_{t}\right)_{t \geq 0}$ and operator semigroup $\left(T_{t}\right)_{t \geq 0}$. We assume that $\psi(\xi)=0$ if and only if $\xi=0$ and that $\frac{1}{\psi} \in L_{l o c}^{2}\left(\mathbb{R}^{n}\right)$, i.e. $\left(\mu_{t}\right)_{t \geq 0}$ and hence $\left(T_{t}\right)_{t \geq 0}$ are transient. From Theorem 5.10 the potential kernel of $\left(\mu_{t}\right)_{t \geq 0}$ is denoted by $\kappa:=F^{-1}\left(\frac{1}{\psi}\right)$ which is on the one hand side a tempered distribution, i.e. $\kappa \in \mathcal{S}^{\prime}\left(\mathbb{R}^{n}\right)$, and on the other hand a measure which is in general unbounded. Indeed, since for $\varepsilon>0$ the function $\xi \mapsto \varepsilon+\psi(\xi)$ is a continuous negative definite function and therefore, see Corollary 1.2.7, $\xi \mapsto \frac{1}{\varepsilon+\psi(\xi)}$ is positive definite, it follows that $\frac{1}{\psi} \in L_{l o c}^{2}\left(\mathbb{R}^{n}\right) \subset \mathscr{D}^{\prime}\left(\mathbb{R}^{n}\right)$ is a positive definite distribution, hence by the Bochner-Schwartz theorem, Theorem 5.2.3, the Fourier transform of $\frac{1}{\psi}$ is a positive measure of at most polynomial growth and can be identified with tempered distributions, see [33].

These posteriori obtained informations allow us to refine the discussions of Chapter 4. Since we know now that $F^{-1}\left(\frac{1}{\psi}\right) \in \mathcal{S}^{\prime}\left(\mathbb{R}^{n}\right)$ we can form immediately the convolution $u:=F^{-1}\left(\frac{1}{\psi}\right) * g$ for $g \in \mathcal{S}\left(\mathbb{R}^{n}\right)$ and we obtain that $\hat{u}=F\left(F^{-1}\left(\frac{1}{\psi}\right) * g\right)=(2 \pi)^{\frac{n}{2}} \frac{1}{\psi} \hat{g}$ and therefore the equality $\psi(\cdot) \hat{u}=(2 \pi)^{\frac{n}{2}} \hat{g}$ is justified. In other words the condition $(1+\psi(\cdot))^{-\frac{s_{0}}{2}} \in L^{2}\left(\mathbb{R}^{n}\right)$ for some $s_{0}>0$ can be removed. It is of utmost importance to note that the general case with $\psi$ being a continuous negative definite function and hence $\frac{1}{\psi}$ being a positive 
definite distribution is not covered by the observations made above. Further, it serves the clarity of the argument to see that certain notions and results are independent of potential theoretical notions, e.g. the singular support is defined for distributions and (4.5) is best understood within the theory of distributions.

We want to take advantage from the knowledge that we can consider $\kappa$ as an element in $\mathcal{S}^{\prime}\left(\mathbb{R}^{n}\right) \cap \mathcal{M}^{+}\left(\mathbb{R}^{n}\right)$. First we note that by the Bochner-Schwartz theorem $\kappa$ is finite on compact sets and for some $M \geq 0$ we know that $\kappa\left(B_{R}(0)\right) \leq c_{0} R^{M}$ for $R \geq 1$. Now let $u: \mathbb{R}^{n} \rightarrow \mathbb{C}$ be a continuous (measurable would be sufficient) function such that for every $N \in \mathbb{N}$ there exists a constant $c_{N} \geq 0$ such that $|u(x)| \leq c_{N}\left(1+|x|^{2}\right)^{-\frac{N}{2}}$. For such a function we have for $R \geq 1$ and $N \in \mathbb{N}$ by Peetre's inequality

$$
\begin{aligned}
\left|\int_{B_{R}^{c}(0)} u(x-y) \kappa(d y)\right| & \leq c_{N} \int_{B_{R}^{c}(0)}\left(1+|x-y|^{2}\right)^{-\frac{N}{2}} \kappa(d y) \\
& \leq 2^{N} c_{N} \int_{B_{R}^{c}(0)} \frac{\left(1+|x|^{2}\right)^{\frac{N}{2}}}{\left(1+|y|^{2}\right)^{\frac{N}{2}}} \kappa(d y),
\end{aligned}
$$

and since $\kappa$ is of at most polynomial growth we can find $N$ such that the integral on the right hand side is finite. Thus we can form $\kappa * g$ for every measurable $g$ which decays faster than every polynomial which is of course a much larger class than $\mathcal{S}\left(\mathbb{R}^{n}\right)$. The more challenging problem is to go a step further: given $\kappa$, find the largest class of measurable functions for which $\kappa * g$ is defined and belongs to some well-understood function space related to $\psi($ or $\psi(D))$. In particular we would like to have $\kappa * g \in H^{\psi, 2}\left(\mathbb{R}^{n}\right)$, a goal which is quite ambitious for a general continuous negative definite function with $\frac{1}{\psi} \in L_{l o c}^{2}\left(\mathbb{R}^{n}\right)$.

Let us have a closer look at the Riesz potentials $R^{(\alpha)} * g$ where $R^{(\alpha)}(x)=$ $c_{n, \alpha}|x|^{\alpha-n}$. By an appropriate choice of $c_{n, \alpha}$ we have $\hat{R}^{(\alpha)}(\xi)=\frac{1}{|\xi|^{\alpha}}$ and hence $F^{-1}\left(\frac{1}{|\cdot|^{\alpha}}\right)(x)=R^{(\alpha)}(x)$. Note that $R^{(\alpha)}$ is for $\alpha>0$ locally integrable since

$$
\int_{0}^{M} r^{\alpha-n} r^{n-1} d r=\frac{M^{\alpha}}{\alpha}
$$

However for $\psi_{\alpha}(\xi)=|\xi|^{\alpha}$ we have

$$
\int_{\overline{B_{M}(0) \backslash B_{\varepsilon}(0)}} \frac{1}{\psi_{\alpha}(\xi)} d \xi=c \int_{\varepsilon}^{M} r^{-\alpha} r^{n-1} d r=\left.\frac{1}{n-\alpha} r^{n-\alpha}\right|_{\varepsilon} ^{M}
$$


and we have to assume $\alpha<\frac{n}{2}$ in order to have $\frac{1}{\psi} \in L_{l o c}^{2}\left(\mathbb{R}^{n}\right)$. Note that for $2<\alpha<\frac{n}{2}$ the function $|\cdot|^{\alpha}$ is not anymore a negative definite function, and $\frac{1}{|\cdot| \alpha}$ is not anymore the limt of positive definite functions, hence a positive definite distribution, but of course the operator $(-\Delta)^{\frac{\alpha}{2}}$ is still well defined on $H^{\alpha}\left(\mathbb{R}^{n}\right)$. Thus in the case of the Riesz potentials and $0<\alpha<2$ we have a locally integrable density $R^{(\alpha)}$ with respect to the Lebesgue measure for $\kappa:=F^{-1}\left(\frac{1}{\psi_{\alpha}}\right)$, i.e.

$$
\kappa=R^{(\alpha)} \lambda^{(n)} .
$$

However, we have to accept that $R^{(\alpha)}(0)$ is either not defined (when we restrict our considerations to real-valued functions) or $R^{(\alpha)}(0)=+\infty$ when allowing functions with values in $\overline{\mathbb{R}}$. The function $R^{(\alpha)}: \mathbb{R}^{n} \rightarrow \overline{\mathbb{R}}$ is lower semicontinuous but $R^{(\alpha)} \notin L^{1}\left(\mathbb{R}^{n}\right)$.

By the example of the Riesz potential we conclude that in some cases the fundamental solution to $\psi(D)$ we can identify with the potential kernel if $\frac{1}{\psi} \in L_{l o c}^{2}\left(\mathbb{R}^{n}\right)$ and this potential kernel has a not necessarily an integrable density with respect to the Lebesgue measure. The following easy to prove result is nonetheless a key theorem in our investigations when starting with our original definition of a fundamental solution.

Theorem 5.3.1. Let $\psi: \mathbb{R}^{n} \rightarrow \mathbb{R}$ be a continuous negative definite function such that $\frac{1}{\psi} \in L_{l o c}^{2}\left(\mathbb{R}^{n}\right)$ and $\psi(\xi) \geq c_{0}|\xi|^{\rho_{0}}$ for all $|\xi| \geq R, \rho_{0}>0$ and $c_{0}>0$. Then the $H^{\psi, s_{0}}$-fundamental solution $E$ to $\psi(D)$ given by $F^{-1}\left(\frac{1}{\psi}\right)$ has a density with respect to the Lebesgue measure which however need not be integrable

Proof. The condition $\psi(\xi) \geq c_{0}|\xi|^{\rho_{0}}$ for all $|\xi| \geq R$ implies that

$$
p_{t}(x):=(2 \pi)^{-n} \int_{\mathbb{R}^{n}} e^{i x \xi} e^{-t \psi(\xi)} d \xi
$$

is an $L^{1}$-density of $\mu_{t}$ where $\left(\mu_{t}\right)_{t \geq 0}$ is the convolution semigroup associated with $\psi$, i.e. $\hat{\mu}_{t}(\xi)=(2 \pi)^{-\frac{n}{2}} e^{-t \psi(\xi)}$. Thus we have $\mu_{t}=p_{t}(\cdot) \lambda^{(n)}$. Since we are in the (symmetric) transient case we have for the potential kernel (5.2), i.e.

$$
\kappa=\int_{0}^{\infty} \mu_{t} d t
$$


implying

$$
k(x):=\int_{0}^{\infty} p_{t}(x) d t
$$

is a density for $\kappa$.

Of course we may replace the condition $\psi(\xi) \geq c_{0}|\xi|^{\rho_{0}}$ for all $|\xi| \geq R$ by the assumption $F^{-1}\left(e^{-t \psi(\cdot)}\right) \in L^{1}\left(\mathbb{R}^{n}\right)$, or more generally by the Hartman-Wintner condition

$$
\lim _{|\xi| \rightarrow \infty} \frac{\psi(\xi)}{\ln (1+|\xi|)}=\infty,
$$

see [27]. However, since the growth conditions for $\psi$ as stated in the theorem will become important later on we prefer to introduce it already in our formulation of the theorem. Thus as a more general result we may state

Theorem 5.3.2. Let $\psi: \mathbb{R}^{n} \rightarrow \mathbb{R}$ be a continuous negative definite function satisfying the Hartman-Wintner condition (5.7) and has the property that $\frac{1}{\psi} \in$ $L_{l o c}^{2}\left(\mathbb{R}^{n}\right)$. Then the pseudo-differential operator $\psi(D)$ has an unique fundamental solution $E \in \mathcal{S}^{\prime}\left(\mathbb{R}^{n}\right)$ given by $E=F^{-1}\left(\frac{1}{\psi}\right)$ and $E$ coincides with the potential kernel $\kappa$ of $\left(\mu_{t}\right)_{t \geq 0}$, the convolution semigroup associated with $\psi$. The potential kernel $\kappa=F^{-1}\left(\frac{1}{\psi}\right)$ has a density $k$ given by (5.6) however in general $k$ does not belong to $L^{1}\left(\mathbb{R}^{n}\right)$.

Before we start to study the density of $\kappa=F^{-1}\left(\frac{1}{\psi}\right)$ we want to discuss some first interesting comparison results for translation invariant extended Dirichlet spaces.

\subsection{Comparison Results for Extended Dirichlet Spaces}

In Proposition 4.3.8 we have seen that if for two continuous negative definite functions $\psi_{1}, \psi_{2}: \mathbb{R}^{n} \rightarrow \mathbb{R}$ we have

$$
\lim _{|\xi| \rightarrow \infty} \frac{\psi_{2}(\xi)}{\psi_{1}(\xi)}=0
$$


then the space $H^{\psi_{1}, s}\left(\mathbb{R}^{n}\right)$ is continuously embedded into the space $H^{\psi_{2}, s}\left(\mathbb{R}^{n}\right)$ and the estimate

$$
\|u\|_{\psi_{2}, s} \leq c_{s}\|u\|_{\psi_{1}, s}
$$

holds for all $u \in H^{\psi_{1}, s}\left(\mathbb{R}^{n}\right)$. Moreover, if for $|\xi| \geq R$ we have with $0<\gamma_{0} \leq \gamma_{1}$ the estimates

$$
\gamma_{0} \leq \frac{\psi_{2}(\xi)}{\psi_{1}(\xi)} \leq \gamma_{1}
$$

the norms $\|u\|_{\psi_{2}, s}$ and $\|u\|_{\psi_{1}, s}$ are equivalent and $H^{\psi_{2}, s}\left(\mathbb{R}^{n}\right)=H^{\psi_{1}, s}\left(\mathbb{R}^{n}\right)$. We may interpret this on the level of the operators $\psi_{1}(D)$ and $\psi_{2}(D)$ as a perturbation result. Suppose that (5.8) holds and $\lim _{|\xi| \rightarrow \infty} \psi_{1}(\xi)=\infty$. Consider the operator $\psi(D)=\psi_{1}(D)+\psi_{2}(D)$. The embedding result implies that $\psi(D)$ is defined on $H^{\psi_{1}, 2}\left(\mathbb{R}^{n}\right)$ and $\psi_{2}(D)$ is an $\eta$-perturbation of $\psi_{1}(D)$ in the sense that for every $\eta>0$ there exists $c(\eta) \in \mathbb{R}$ such that

$$
\left\|\psi_{2}(D) u\right\|_{L^{2}} \leq \eta\left\|\psi_{1}(D) u\right\|_{L^{2}}+c(\eta)\|u\|_{L^{2}}
$$

holds for all $u \in H^{\psi_{1}, 2}\left(\mathbb{R}^{n}\right)$. Hence $\left(\psi_{1}(D)+\psi_{2}(D), H^{\psi_{1}, 2}\left(\mathbb{R}^{n}\right)\right)$ is closed and a generator of an $L^{2}$ sub-Markovian semigroup. Moreover, for the corresponding Dirichlet forms $\mathcal{E}^{\psi}$ and $\mathcal{E}^{\psi_{1}}$ we have

$$
\mathcal{E}^{\psi_{1}}(u, u) \leq \mathcal{E}^{\psi}(u, u) \text { for all } u \in H^{\psi_{1}, 1}\left(\mathbb{R}^{n}\right)
$$

If for $\mathcal{E}^{\psi_{1}}$ a Nash-type inequality holds, i.e. if for some $p>2$ we have

$$
\|u\|_{L^{p}}^{2} \leq c \mathcal{E}^{\psi_{1}}(u, u) \text { for all } u \in H^{\psi_{1}, 1}\left(\mathbb{R}^{n}\right)
$$

the same holds for $\mathcal{E}^{\psi}$ and consequently bounds for $\left\|T_{t}^{\psi_{1}}\right\|_{L^{1}-L^{\infty}}$ carry over to $\left\|T_{t}^{\psi}\right\|_{L^{1}-L^{\infty}}$, see [40] and [41] or [21] for a detailed discussion. Let us give a more careful analysis of the proof of (5.9). The condition (5.8) yields that for every $\eta>0$ the existence of $R(\eta) \geq 0$ such that $|\xi| \geq R(\eta)$ implies $\psi_{2}(\xi) \leq \eta \psi_{1}(\xi)$, and now we find

$$
\begin{aligned}
\|u\|_{\psi_{2}, s}^{2} & =\int_{\mathbb{R}^{n}}\left(1+\psi_{2}(\xi)\right)^{s}|\hat{u}(\xi)|^{2} d \xi \\
& =\int_{|\xi| \leq R(\eta)}\left(1+\psi_{2}(\xi)\right)^{s}|\hat{u}(\xi)|^{2} d \xi+\int_{|\xi| \geq R(\eta)}\left(1+\psi_{2}(\xi)\right)^{s}|\hat{u}(\xi)|^{2} d \xi
\end{aligned}
$$


Only the second term is of interest since the first term is immediately estimated against $\|u\|_{L^{2}}^{2}$, or with more accuracy we have

$$
\int_{|\xi| \leq R(\eta)}\left(1+\psi_{2}(\xi)\right)^{s}|\hat{u}(\xi)|^{2} d \xi \leq \max _{|\xi| \leq R(\eta)}(1+\psi(\xi))^{s}\|u\|_{L^{2}}^{2}
$$

and of course we have $\|u\|_{\psi_{1}, s}^{2} \sim\|u\|_{L^{2}}^{2}+\|u\|_{\psi_{1}, s}^{2}$. The handling of the second term however requires the estimate $1+\psi_{2}(\xi) \leq \eta\left(1+\psi_{1}(\xi)\right)$ for $|\xi| \geq R(\eta)$ and this leads to

$$
\int_{|\xi| \geq R(\eta)}\left(1+\psi_{2}(\xi)\right)^{s}|\hat{u}(\xi)|^{2} d \xi \leq \eta^{s} \int_{|\xi| \geq R(\eta)}\left(1+\psi_{1}(\xi)\right)^{s}|\hat{u}(\xi)|^{2} d \xi \leq \eta^{s}\|u\|_{\psi_{1}, s}^{2}
$$

An easy example is given by the two continuous negative definite functions $\psi_{1}(\xi)=|\xi|^{\alpha}$ and $\psi_{2}(\xi)=|\xi|^{\beta}$ with $\alpha>\beta$. We emphasize that we need no condition for the behaviour of $\frac{\psi_{2}(\xi)}{\psi_{1}(\xi)}$ as $\xi$ tends to 0 , but we note that while $|\xi|^{\beta} \leq|\xi|^{\alpha}$ for $0<\beta<\alpha$ and $|\xi| \geq 1$, for $|\xi| \leq 1$ this inequality reverses, i.e. we have now $|\xi|^{\alpha} \leq|\xi|^{\beta}$. When working with the extended Dirichlet spaces of two transient Dirichlet spaces this observation becomes important.

Let $\psi_{1}, \psi_{2}: \mathbb{R}^{n} \rightarrow \mathbb{R}$ be two continuous negative definite functions such that their only zero is $\xi=0$, and suppose that $\frac{1}{\psi_{1}}, \frac{1}{\psi_{2}} \in L_{l o c}^{1}\left(\mathbb{R}^{n}\right)$. Denote by $H_{e}^{\psi_{1}, 1}\left(\mathbb{R}^{n}\right)$ and $H_{e}^{\psi_{2}, 1}\left(\mathbb{R}^{n}\right)$ the corresponding extended Dirichlet spaces, i.e.

$$
H_{e}^{\psi_{j}, 1}\left(\mathbb{R}^{n}\right)=\left\{u \in L_{l o c}^{1}\left(\mathbb{R}^{n}\right) \mid \psi_{j} \hat{u} \in L^{2}\left(\mathbb{R}^{n}\right)\right\},
$$

and corresponding (extended) Dirichlet form by

$$
\mathcal{E}^{\psi_{j}}(u, v)=\int_{\mathbb{R}^{n}} \psi_{j}(\xi) \hat{u}(\xi) \overline{\hat{v}(\xi)} d \xi
$$

We now add the assumptions that for $j=1,2$

$$
c_{j}|\xi|^{\alpha_{j}} \leq \psi_{j}(\xi)
$$

holds for all $\psi \in \mathbb{R}^{n}$, which by Corollary 5.1 .14 implies that

$$
H_{e}^{\psi_{j}, 1}\left(\mathbb{R}^{n}\right) \subset L^{p_{j}}\left(\mathbb{R}^{n}\right), p_{j}=\frac{2 n}{n-\alpha_{j}}, n \geq 2,
$$

and

$$
\|u\|_{L^{p_{j}}}^{2} \leq \tilde{c}_{j} \mathcal{E}^{\psi_{j}}(u, u)
$$


holds for all $u \in H_{e}^{\psi_{j}, 1}\left(\mathbb{R}^{n}\right)$. In particular we find that now $\mathcal{E}^{\psi_{j}}(u, v)$ is a scalar product on $H_{e}^{\psi_{j}, 1}\left(\mathbb{R}^{n}\right)$ and we denote the corresponding norm sometimes by

$$
\|u\|_{H_{e}^{\psi_{j}, 1}}^{2}=\mathcal{E}^{\psi_{j}}(u, u)
$$

The estimate

$$
\psi_{2}(\xi) \leq \gamma_{0} \psi_{1}(\xi) \text { for all } \xi \in \mathbb{R}^{n}
$$

implies immediately

$$
\mathcal{E}^{\psi_{2}}(u, u) \leq \gamma_{0} \mathcal{E}^{\psi_{1}}(u, u) \text { for all } u \in H_{e}^{\psi_{1}, 1}\left(\mathbb{R}^{n}\right),
$$

however we cannot relax (5.11) to an inequality to hold only for all $|\xi| \geq R$ for some $R>0$. This is a dramatic change compared with the case of the two forms $\mathcal{E}_{1}^{\psi_{j}}(u, u):=\mathcal{E}^{\psi_{j}}(u, u)+(u, u)_{L^{2}}$. A more detailed analysis shows that we need the following two conditions

$$
\psi_{2}(\xi) \leq \omega_{1} \psi_{1}(\xi) \text { for all }|\xi| \leq \rho_{1}, \rho_{1}>0
$$

and

$$
\psi_{2}(\xi) \leq \omega_{2} \psi_{1}(\xi) \text { for all }|\xi| \geq \rho_{2}, \rho_{2} \geq \rho_{1} .
$$

Indeed under (5.12) and (5.13) we find

$$
\begin{aligned}
\mathcal{E}^{\psi_{2}}(u, u) & =\int_{\mathbb{R}^{n}} \psi_{2}(\xi)|\hat{u}(\xi)|^{2} d \xi \\
& =\int_{|\xi| \leq \rho_{1}} \psi_{2}(\xi)|\hat{u}(\xi)|^{2} d \xi+\int_{\rho_{1}<|\xi| \leq \rho_{2}} \psi_{2}(\xi)|\hat{u}(\xi)|^{2} d \xi \\
& +\int_{\rho_{2} \leq|\xi|} \psi_{2}(\xi)|\hat{u}(\xi)|^{2} d \xi \\
& \leq \omega_{1} \int_{|\xi| \leq \rho_{1}} \psi_{1}(\xi)|\hat{u}(\xi)|^{2} d \xi+\int_{\rho_{1}<|\xi| \leq \rho_{2}} \psi_{2}(\xi)|\hat{u}(\xi)|^{2} d \xi \\
& +\omega_{2} \int_{\rho_{2} \leq|\xi|} \psi_{1}(\xi)|\hat{u}(\xi)|^{2} d \xi .
\end{aligned}
$$

On the compact set $\overline{B_{\rho_{2}}(0)} \backslash B_{\rho_{1}}(0)$ the continuous function $\psi_{2}$ is bounded by $\left\|\psi_{2}\right\|_{\infty, \overline{B_{\rho_{2}}(0)} \backslash B_{\rho_{1}}(0)}$ and the continuous function $\psi_{1}$ which has by assumption its only zero at 0 is bounded on $\overline{B_{\rho_{2}}(0)} \backslash B_{\rho_{1}}(0)$ from below by

$$
\mathcal{M}_{\psi_{1}}:=\inf _{\xi \in \overline{B_{\rho_{2}}(0)} \backslash B_{\rho_{1}}(0)} \psi_{1}(\xi)>0 .
$$


Thus $\overline{B_{\rho_{2}}(0)} \backslash B_{\rho_{1}}(0)$ we have

$$
\psi_{2}(\xi) \leq\left\|\psi_{2}\right\|_{\infty, \overline{B_{\rho_{2}}(0)} \backslash B_{\rho_{1}}(0)} \leq\left\|\psi_{2}\right\|_{\infty, \overline{B_{\rho_{2}}(0)} \backslash B_{\rho_{1}}(0)} \cdot \frac{\psi_{1}(\xi)}{\mathcal{M}_{\psi_{1}}}
$$

i.e.

$$
\psi_{2}(\xi) \leq c_{\psi_{1}, \psi_{2}, \rho_{1}, \rho_{2}} \psi_{1}(\xi) \text { for } \xi \in \overline{B_{\rho_{2}}(0)} \backslash B_{\rho_{1}}(0)
$$

and we arrive at

$$
\int_{\rho_{1}<|\xi| \leq \rho_{2}} \psi_{2}|\hat{u}(\xi)|^{2} d \xi \leq c_{\psi_{1}, \psi_{2}, \rho_{1}, \rho_{2}} \int_{\rho_{1}<|\xi| \leq \rho_{2}} \psi_{1}(\xi)|\hat{u}(\xi)|^{2} d \xi .
$$

Hence we have proved

Theorem 5.4.1. Suppose that the continuous negative definite functions $\psi_{1}, \psi_{2}$ : $\mathbb{R}^{n} \rightarrow \mathbb{R}$ satisfy our standard condition, i.e. $\xi=0$ is their only zero, $\frac{1}{\psi_{j}} \in$ $L_{\text {loc }}^{2}\left(\mathbb{R}^{n}\right)$ and (5.10). In addition assume (5.12) and (5.13). Then we have for all $u \in H_{e}^{\psi_{1}, 1}\left(\mathbb{R}^{n}\right)$ the estimate

$$
\mathcal{E}^{\psi_{2}}(u, u) \leq \gamma \mathcal{E}^{\psi_{1}}(u, u)
$$

and $H_{e}^{\psi_{1}, 1}\left(\mathbb{R}^{n}\right)$ is continuously embedded into $H_{e}^{\psi_{2}, 1}\left(\mathbb{R}^{n}\right)$.

This theorem has the

Corollary 5.4.2. Under the assumptions of Theorem 5.4.1 the space $H_{e}^{\psi_{1}, 1}\left(\mathbb{R}^{n}\right)$ is continuously embedded into the space $L^{p_{1}}\left(\mathbb{R}^{n}\right) \cap L^{p_{2}}\left(\mathbb{R}^{n}\right)$ with $p_{j}=\frac{2 n}{n-\alpha_{j}}, n \geq 2$.

We observe that under the conditions of Theorem 5.4.1 we can obtain Nashtype estimates and consequently diagonal estimates for the sub-Markovian semigroups $\left(T_{t}^{\psi_{j}}\right)_{t \geq 0}$, in fact we can obtain comparison results for $\left\|T_{t}^{\psi_{j}}\right\|_{L^{1}-L^{\infty}}$, see [41] or [21]. We want to emphasize once more that for comparing translation invariant Dirichlet spaces a comparison of the symbols at $+\infty$ is needed, however in the case of translation invariant extended Dirichlet spaces (if they exist) we need to compare the symbols at $+\infty$ and at 0 . (We use the term symbol here for the corresponding continuous negative definite functions since they are symbols of the generators considered as pseudo-differential operators.) 
We return to the spaces $H^{\psi_{1}, 1}\left(\mathbb{R}^{n}\right)$ and $H^{\psi_{2}, 1}\left(\mathbb{R}^{n}\right)$. With $\tau(\xi):=\frac{\left(1+\psi_{1}(\xi)\right)^{\frac{1}{2}}}{\left(1+\psi_{2}(\xi)\right)^{\frac{1}{2}}}$ we find that $\tau(D): H^{\psi_{1}, 1}\left(\mathbb{R}^{n}\right) \rightarrow H^{\psi_{2}, 1}\left(\mathbb{R}^{n}\right)$ is an isometry

$$
\|\tau(D) u\|_{\psi_{2}, 1}=\left\|\tau(\cdot)\left(1+\psi_{2}(\cdot)\right)^{\frac{1}{2}} \hat{u}\right\|_{L^{2}}=\left\|\left(1+\psi_{1}(\cdot)\right)^{\frac{1}{2}} \hat{u}\right\|_{L^{2}}=\|u\|_{\psi_{1}, 1},
$$

and this isometry has a well defined inverse $\tau(D)^{-1}: H^{\psi_{2}, 1}\left(\mathbb{R}^{n}\right) \rightarrow H^{\psi_{1}, 1}\left(\mathbb{R}^{n}\right)$ with symbol $\left(\frac{1+\psi_{2}(\xi)}{1+\psi_{1}(\xi)}\right)^{\frac{1}{2}}$.

In the situation where $\psi_{1}$ and $\psi_{2}$ satisfy our standard assumptions for constructing the extended Dirichlet spaces $H_{e}^{\psi_{j}, 1}\left(\mathbb{R}^{n}\right) \subset L^{p_{j}}\left(\mathbb{R}^{n}\right)$, and under the condition

$$
\psi_{2}(\xi) \leq \gamma_{0} \psi_{1}(\xi) \text { for all } \xi \in \mathbb{R}^{n}
$$

we can conclude that $0 \leq \frac{\psi_{2}(\xi)}{\psi_{1}(\xi)} \leq \gamma_{0}$ and with

$$
0 \leq \sigma(\xi) \leq\left(\frac{\psi_{2}(\xi)}{\psi_{1}(\xi)}\right)^{\frac{1}{2}} \leq \sqrt{\gamma_{0}}
$$

we deduce

$$
\|\sigma(D) u\|_{H_{e}^{\psi_{1}, 1}}=\left\|\sigma(\cdot) \psi_{1}^{\frac{1}{2}}(\cdot) \hat{u}\right\|_{L^{2}}=\left\|\psi_{2}^{\frac{1}{2}}(\cdot) \hat{u}\right\|_{L^{2}}=\|u\|_{H_{e}^{\psi_{2}, 1}}
$$

i.e. $\sigma(D): H_{e}^{\psi_{1}, 1}\left(\mathbb{R}^{n}\right) \rightarrow H_{e}^{\psi_{2}, 1}\left(\mathbb{R}^{n}\right)$ is an isometry. However we have difficulties to form $\sigma^{-1}(\xi)=\frac{\psi_{1}(\xi)}{\psi_{2}(\xi)}$ since this expression might be undefined at $\xi=0$. We need only conisder $\psi_{2}(\xi)=|\xi|^{\alpha}$ and $\psi_{1}(\xi)=|\xi|^{\alpha}+|\xi|^{\beta}$ with $\alpha>\beta$. It follows that $\psi_{2}(\xi) \leq \psi_{1}(\xi)$ for all $\xi \in \mathbb{R}^{n}$ and if $\xi \neq 0$ we find

$$
0 \leq \frac{|\xi|^{\alpha}}{|\xi|^{\alpha}+|\xi|^{\beta}}=\frac{1}{1+|\xi|^{\beta-\alpha}} \leq 1 .
$$

Note that $\lim _{|\xi| \rightarrow 0}|\xi|^{\beta-\alpha}=\infty$ and therefore we can continuously extend $\xi \mapsto$ $\frac{|\xi|^{\alpha}}{|\xi|^{\alpha}+|\xi|^{\beta}}$ at $\xi=0$ by the value 0 . On the other hand we have for $\xi \neq 0$ that

$$
0 \leq \frac{|\xi|^{\alpha}+|\xi|^{\beta}}{|\xi|^{\alpha}}=1+|\xi|^{\beta-\alpha}
$$

and since $\lim _{\xi \rightarrow 0}|\xi|^{\beta-\alpha}=+\infty$ for $\alpha>\beta$ this quotient is unbounded at $\xi=0$, i.e. it cannot be extended to a well behaved symbol.

Let us observe that for $u \in \mathcal{S}\left(\mathbb{R}^{n}\right)\left(\right.$ or $\left.L^{1}\left(\mathbb{R}^{n}\right)\right)$ we have

$$
\hat{u}(0)=(2 \pi)^{-\frac{n}{2}} \int_{\mathbb{R}^{n}} u(x) d x,
$$


which means that up to a factor $\hat{u}(0)$ is the mean-value of $u \in \mathcal{S}\left(\mathbb{R}^{n}\right)\left(\right.$ or $\left.L^{1}\left(\mathbb{R}^{n}\right)\right)$. Let us denote the mean-value of $u \in L^{1}\left(\mathbb{R}^{n}\right)$ by $u_{\mathcal{M}}$. Suppose $v \in L^{1}\left(\mathbb{R}^{n}\right)$ has mean-value zero and $\varphi: \mathbb{R}^{n} \backslash\{0\} \rightarrow \mathbb{R}$ is a non-negative continuous function with $\lim _{|\xi| \rightarrow \infty} \varphi(\xi)=+\infty$. In this case we can define $\varphi(\xi) \hat{v}(\xi)$ for $\xi \neq 0$ and we may extend this to $\xi=0$ by setting $(\varphi \hat{v})(0)=0$. Now let $\psi_{1}$ and $\psi_{2}$ satisfy our standard conditions and in addition (5.14). For $u \in \mathcal{S}\left(\mathbb{R}^{n}\right)$ such that $u_{\mathcal{M}}=0$ the term

$$
\hat{v}(\xi):= \begin{cases}\frac{\psi_{1}^{\frac{1}{2}}(\xi)}{\psi_{2}^{\frac{1}{2}}(\xi)} \psi_{2}^{\frac{1}{2}}(\xi) \hat{u}(\xi), & \xi \neq 0 \\ 0, & \xi=0\end{cases}
$$

is well defined and an element in $L^{2}\left(\mathbb{R}^{n}\right)$. Moreover we find

$$
\|\hat{v}\|_{L^{2}}=\left\|\psi_{1}^{\frac{1}{2}} \hat{u}\right\|_{L^{2}}=\|u\|_{H_{e}^{\psi_{1}, 1}}
$$

Denote by $H_{e, 0}^{\psi_{2}, 1}\left(\mathbb{R}^{n}\right)$ the closure in $H_{e}^{\psi_{2}, 1}\left(\mathbb{R}^{n}\right)$ of the space $\left\{u \in H_{e}^{\psi_{2}, 1}\left(\mathbb{R}^{n}\right) \cap\right.$ $\left.L^{2}\left(\mathbb{R}^{n}\right) \cap L^{1}\left(\mathbb{R}^{n}\right) \mid u_{\mathcal{M}}=0\right\}$. For $u \in H_{e, 0}^{\psi_{2}, 1}\left(\mathbb{R}^{n}\right)$ we can define $\sigma^{-1}(D)$ by

$$
\sigma^{-1}(D) u=F^{-1}\left(\frac{\psi_{1}^{\frac{1}{2}}}{\psi_{2}^{\frac{1}{2}}} \hat{u}\right)
$$

and it follows that $\sigma^{-1}(D): H_{e, 0}^{\psi_{2}, 1}\left(\mathbb{R}^{n}\right) \rightarrow H_{e}^{\psi_{1}, 1}\left(\mathbb{R}^{n}\right)$ is an isometry.

Finally we want to mention that it is possible to define higher order extended Dirichlet spaces by

$$
H_{e}^{\psi, k}\left(\mathbb{R}^{n}\right):=\left\{u \in H_{e}^{\psi, k-1}\left(\mathbb{R}^{n}\right) \mid \psi^{\frac{k}{2}} \hat{u} \in L^{2}\left(\mathbb{R}^{n}\right)\right\}
$$

but we will not follow this idea further.

Let us return to the operator (2.11), i.e.

$$
q\left(x, D_{x}\right) u(x)=a(y) \psi_{1}\left(D_{z}\right) u(x)+b(z) \psi_{2}\left(D_{y}\right) u(x) .
$$

From the proof of Theorem 2.4.7 we deduce

$$
|B(u, v)| \leq c\left\|\psi^{\frac{1}{2}}(D) u\right\|_{L^{2}}\left\|\psi^{\frac{1}{2}}(D) v\right\|_{L^{2}}
$$

and moreover, from the proof of Theorem 2.4.8, i.e. (2.13), we obtain

$$
B(u, u) \geq \kappa\left\|\psi^{\frac{1}{2}}(D) u\right\|_{L^{2}}^{2},
$$


or

$$
B(u, u) \geq \kappa \int \psi(\xi)|\hat{u}(\xi)|^{2} d \xi
$$

Now, if the Dirichlet form $\left(\mathcal{E}^{\psi}, H^{\psi, 1}\left(\mathbb{R}^{n}\right)\right)$ is transient we find the estimate

$$
(B(u, u))^{\frac{1}{2}} \geq\left(\kappa \mathcal{E}^{\psi}(u, u)\right)^{\frac{1}{2}} \geq \kappa^{\frac{1}{2}} \int_{\mathbb{R}^{n}} g|u| d x
$$

or

$$
\int \tilde{g}|u| d x \leq B(u, u)^{\frac{1}{2}}
$$

for a strictly positive and bounded function $\tilde{g}=\sqrt{\kappa} g \in L^{1}\left(\mathbb{R}^{n}\right)$, hence $\left(B, H^{\psi, 1}\left(\mathbb{R}^{n}\right)\right)$ is a transient symmetric Dirichlet form too.

We may also consider the operator $q\left(x_{0}, D_{x}\right)$ with frozen coefficients, i.e.

$$
q\left(x_{0}, D_{x}\right) u(x)=a\left(y_{0}\right) \psi_{1}\left(D_{z}\right) u(x)+b\left(z_{0}\right) \psi_{2}\left(D_{y}\right) u(x) .
$$

This operator gives rise to a translation invariant symmetric Dirichlet form $\left(\mathcal{E}^{q\left(x_{0}, \cdot\right)}, H^{\psi, 1}\left(\mathbb{R}^{n}\right)\right)$ and again we may use $(5.15)$ to deduce that $\left(\mathcal{E}^{q\left(x_{0}, \cdot\right)}, H^{\psi, 1}\left(\mathbb{R}^{n}\right)\right)$ is previously developed, in particular those related to its fundamental solution and potential kernel $k\left(x_{0}, \xi\right)$. Of great intereset is to which extent one can use $k\left(x_{0}, \xi\right)$ as an approximation of a parametrix to the pseudo-differential operator $q(x, D)$, a topic which must be postponed to later investigations.

In Chapter 6 we will relate these comparison results to comparisons of potential kernels, i.e. fundamental solution. 


\section{Chapter 6}

\section{Potential Kernels and Intrinsic Metrics}

\subsection{Some First Properties of Potential Kernels}

Throughout this chapter $\psi: \mathbb{R}^{n} \rightarrow \mathbb{R}$ is a continuous negative definite function such that

i) $\psi(\xi)=0$ if and only if $\xi=0$;

ii) $\frac{1}{\psi} \in L_{l o c}^{1}\left(\mathbb{R}^{n}\right)$;

iii) for some $c_{0}>0,0<\gamma_{0}<2$, and $R_{0} \geq 0$ we have

$$
c_{0}|\xi|^{\gamma_{0}} \leq \psi(\xi) \text { for }|\xi| \geq R_{0}
$$

iv) for some $c_{1} \geq 0,0<\gamma_{1}<2$, and $R_{1} \geq 0$ we have

$$
\psi(\xi) \leq c_{1}|\xi|^{\gamma_{1}} \text { for }|\xi| \geq R_{1}
$$

Note that (6.2) is equivalent to

$$
(1+\psi(\xi))^{\frac{1}{2}} \leq \tilde{c_{1}}\left(1+|\xi|^{2}\right)^{\frac{\gamma_{1}}{2}} \text { for all } \xi \in \mathbb{R}^{n}
$$


We know that $k:=F^{-1}\left(\frac{1}{\psi}\right) \in \mathcal{S}^{\prime}\left(\mathbb{R}^{n}\right) \cap \mathcal{M}_{+}\left(\mathbb{R}^{n}\right)$ and

$$
k=k(\cdot) \lambda^{(n)}
$$

but in general $k \notin L^{1}\left(\mathbb{R}^{n}\right)$.

Note that once we want to interpret $F^{-1}\left(\frac{1}{\psi}\right)$ as a fundamental solution we need to add the assumption $\frac{1}{\psi} \in L_{l o c}^{2}\left(\mathbb{R}^{n}\right)$.

Corollary 6.1.1. Under the assumptions stated above $k$ is an even function and if $\psi$ is rotational invariant then $k$ is rotational invariant too.

Moreover we have

Lemma 6.1.2. Under the assumptions stated above we have for $\gamma_{1} \leq n$ that

$$
k(0)=\lim _{r \rightarrow \infty} \int_{B_{r}(0)} \frac{1}{\psi(\xi)} d \xi=+\infty .
$$

Proof. For $r>R_{1}$ we have

$$
\begin{aligned}
\int_{\overline{B_{r}(0)}} \frac{1}{\psi(\xi)} d \xi & \geq \int_{\overline{B_{r}(0) \backslash B_{R_{1}}(0)}} \frac{1}{\psi(\xi)} d \xi \geq \frac{1}{c_{1}} \int_{\overline{B_{r}(0)} \backslash B_{R_{1}}(0)} \frac{1}{|\xi|^{\gamma_{1}}} d \xi \\
& =\frac{\omega_{n}}{c_{1}} \int_{R^{1}}^{r} \rho^{n-1-\gamma_{1}} d \rho \\
& =\frac{\omega_{n}}{c_{1}}\left\{\begin{array}{lc}
\left.\frac{1}{n-\gamma_{1}} \rho^{n-\gamma_{1}}\right|_{R^{1}} ^{r}, & n \neq \gamma_{1} \\
\left.\ln \rho\right|_{R^{1}} ^{r}, & n=\gamma_{1}
\end{array}\right.
\end{aligned}
$$

and therefore for $\gamma_{1} \leq n$ it follows that

$$
\lim _{r \rightarrow \infty} \int_{\frac{B_{r}(0)}{\psi(\xi)}} \frac{1}{\psi(\xi=+\infty}
$$

We want to investigate the additional condition $k \in L_{l o c}^{2}\left(\mathbb{R}^{n} \backslash\{0\}\right)$. From (6.1) it follows that $e^{-t \psi} \in L^{1}\left(\mathbb{R}^{n}\right) \cap C_{b}\left(\mathbb{R}^{n}\right)$ and therefore $\mu_{t}$ has the density $p_{t} \in C_{\infty}\left(\mathbb{R}^{n}\right)$ where

$$
p_{t}(x)=(2 \pi)^{-n} \int_{\mathbb{R}^{n}} e^{i x \xi} e^{i t \psi(\xi)} d \xi
$$


and $\left(\mu_{t}\right)_{t \geq 0}$ is the convolution semigroup associated with $\psi$ by the relation

$$
\hat{\mu}_{t}(\xi)=(2 \pi)^{-\frac{n}{2}} e^{-t \psi(\xi)}
$$

It follows that

$$
k(x)=\int_{0}^{\infty} p_{t}(x) d t .
$$

For $\rho>0$ we find further by Tonelli's theorem that

$$
\begin{aligned}
\int_{\overline{B_{\rho}(0)}} k(x) d x & =\int_{\mathbb{R}^{n}} \chi_{\overline{B_{\rho}(0)}}(x)\left(\int_{0}^{\infty} p_{t}(x) d t\right) d x \\
& =\int_{0}^{\infty}\left(\int_{\mathbb{R}^{n}} p_{t}(x) \chi_{\overline{B_{\rho}(0)}}(x) d x\right) d t \\
& =\int_{0}^{\infty}\left(T_{t} \chi_{\overline{B_{\rho}(0)}}\right)(0) d t
\end{aligned}
$$

From (6.1) it follows that

$$
\|u\|_{L^{p}}^{2} \leq c \mathcal{E}(u, u) \text { for all } u \in H^{\psi, 1}\left(\mathbb{R}^{n}\right)
$$

where $p=\frac{2 n}{n-\gamma_{0}}$, compare with Corollary 5.1.13. A well known result, see [41] or [21], states that (6.3) is equivalent to

$$
\left\|T_{t}\right\|_{L^{1}-L^{\infty}} \leq \tilde{c} t^{-\frac{N}{2}}, t>0
$$

where $N=\frac{2 p}{p-2}>2$, i.e. under the assumption (6.1) we have

$$
N=\frac{2 n}{\gamma_{0}} \text { provided } n>\frac{\gamma_{0}}{2}
$$

Here $\left\|T_{t}\right\|_{L^{1}-L^{\infty}}$ denotes the $L^{1}-L^{\infty}$-operator norm of the extension of the $L^{2}$ sub-Markovian semigroup associated with $\left(\mu_{t}\right)_{t \geq 0}$ or equivalently $\psi$. Therefore we can conclude

$$
\begin{aligned}
\int_{0}^{\infty}\left(T_{t} \chi_{\overline{B_{\rho}(0)}}\right)(0) d t & =\int_{0}^{1}\left(T_{t} \chi_{\overline{B_{\rho}(0)}}\right)(0) d t+\int_{1}^{\infty}\left(T_{t} \chi_{\overline{B_{\rho}(0)}}\right)(0) d t \\
& \leq \int_{0}^{1}\left\|T_{t}\right\|_{L^{2}-L^{2}}\left\|\chi_{\overline{B_{\rho}(0)}}\right\|_{L^{2}} d t+\int_{1}^{\infty}\left\|T_{t}\right\|_{L^{1}-L^{\infty}} \| \chi_{\overline{B_{\rho}(0)} \|_{L^{1}}} d t \\
& \leq \lambda^{(n)}\left(\overline{B_{\rho}(0)}\right)^{\frac{1}{2}}+\tilde{c} \int_{1}^{\infty} t^{-\frac{2 n}{\gamma_{0}}} d t \lambda^{(n)}\left(\overline{B_{\rho}(0)}\right) \\
& =\lambda^{(n)}\left(\overline{B_{\rho}(0)}\right)^{\frac{1}{2}}+\tilde{c} \lambda^{(n)}\left(\overline{B_{\rho}(0)}\right)\left(\left.\frac{\gamma_{0}}{\gamma_{0}-2 n} t^{\frac{\gamma_{0}-2 n}{\gamma_{0}}}\right|_{1} ^{\infty}\right) \\
& =\lambda^{(n)}\left(\overline{B_{\rho}(0)}\right)^{\frac{1}{2}}+\tilde{c} \lambda^{(n)}\left(\overline{B_{\rho}(0)}\right) \frac{\gamma_{0}}{2 n-\gamma_{0}}
\end{aligned}
$$


provided $\gamma_{0}<2 n$. In this calculation we also used that $T_{t}$ is an $L^{2}$-contraction, i.e. $\left\|T_{t}\right\|_{L^{2}-L^{2}} \leq 1$. Thus we have proved

Theorem 6.1.3. Under the assumptions i)-iv) and the additional conditions $\gamma_{0}<2 n$ and $\gamma_{1} \leq n$ the potential kernel $k=F^{-1}\left(\frac{1}{\psi}\right)$ has the following properties

i) $k(0)=\infty$;

ii) $k \in L_{l o c}^{2}\left(\mathbb{R}^{n} \backslash\{0\}\right)$.

Moreover, $k$ is an even function and rotational symmetric if $\psi$ is.

Before we continue to study $k$ further, we want to discuss several examples. We want to provide some examples of continuous negative definite functions satisfying the conditions of Theorem 4.4.4.

\subsection{Examples}

Examples 6.2.1-6.2.8 are taken from [20] or are obtained by subordination using the discussion in [35]. We begin with those examples involving one variable.

Example 6.2.1. Consider the continuous negative definite function $\psi(\xi)=|\xi|^{\alpha}$, $0<\alpha<2$. For $n=1$ we obtain

$$
\int_{-1}^{1}|\xi|^{-\alpha} d \xi=2 \int_{0}^{1} \xi^{-\alpha} d \xi=\left.\frac{1}{1-\alpha} \xi^{1-\alpha}\right|_{0} ^{1} .
$$

Therefore $\frac{1}{|\xi|^{\alpha}} \in L_{\text {loc }}^{1}\left(\mathbb{R}^{n}\right)$ if and only if $1-\alpha>0$, i.e. $\alpha<1$ which implies $\frac{1}{|\xi|^{\alpha}} \in L_{l o c}^{2}\left(\mathbb{R}^{n}\right)$ if and only if $\alpha<\frac{1}{2}$. Moreover for $n=2$ we obtain

$$
\begin{aligned}
\int_{B_{1}(0)}|\xi|^{-\alpha} d \xi & =2 \pi \int_{0}^{1} r^{-\alpha} r d r=2 \pi \int_{0}^{1} r^{1-\alpha} d r \\
& =\left.2 \pi \frac{1}{2-\alpha} r^{2-\alpha}\right|_{0} ^{1}
\end{aligned}
$$


Therefore in this case we require $2-\alpha>0$, i.e. $\alpha<2$, for local integrability and $\frac{1}{|\xi|^{\alpha}} \in L_{l o c}^{2}\left(\mathbb{R}^{n}\right)$ if and only if $\alpha<1$. Moreover, for higher dimensions, i.e. $n \geq 3$, we have

$$
\begin{aligned}
\int_{B_{1}(0)}|\xi|^{-\alpha} d \xi & =c_{n} \int_{0}^{1} r^{-\alpha} r^{n-1} d r=c_{n} \int_{0}^{1} r^{n-1-\alpha} d r \\
& =\left.c_{n} \frac{1}{n-\alpha} r^{n-\alpha}\right|_{0} ^{1}
\end{aligned}
$$

Hence given that $n-\alpha>0$, i.e. $\alpha<n$ we can conclude that $\frac{1}{|\xi|^{\alpha}} \in L_{l o c}^{1}\left(\mathbb{R}^{n}\right)$. Moreover $\frac{1}{|\xi|^{\alpha}} \in L_{\text {loc }}^{2}\left(\mathbb{R}^{n}\right)$ if and only if $\alpha<\frac{n}{2}$ and therefore the conditions in Theorem 4.4.6 are satisfied.

Example 6.2.2. Consider the continuous negative definite function $\psi(\xi)=$ $\frac{|\xi|^{\alpha}}{1+|\xi|^{\alpha}}$. Firstly we state that in general

$$
\frac{s}{1+s} \geq \frac{s}{2} \text { for } s<1 \text {. }
$$

Therefore we can estimate

$$
\int_{B_{1}(0)}\left(\frac{|\xi|^{\alpha}}{1+|\xi|^{\alpha}}\right)^{-1} d \xi \leq 2 \int_{B_{1}(0)}|\xi|^{-\alpha} d \xi
$$

Hence $\left(\frac{|\xi|^{\alpha}}{1+|\xi|^{\alpha}}\right)^{-1} \in L_{l o c}^{1}\left(\mathbb{R}^{n}\right)$ given the following situations:

$$
\begin{aligned}
& n=1: \alpha<1 ; \\
& n=2: \alpha<2 ; \\
& n \geq 3: \alpha<n .
\end{aligned}
$$

Moreover $\left(\frac{|\xi|^{\alpha}}{1+|\xi|^{\alpha}}\right)^{-1} \in L_{\text {loc }}^{2}\left(\mathbb{R}^{n}\right)$ given the following situations:

$$
\begin{aligned}
& n=1: \alpha<\frac{1}{2} \\
& n=2: \alpha<1 \\
& n \geq 3: \alpha<\frac{n}{2} .
\end{aligned}
$$


Example 6.2.3. Consider the continuous negative definite function $\psi(\xi)=$ $|\xi|^{\frac{\alpha}{2}} \arctan |\xi|^{\frac{\alpha}{2}}$. In general we have for $0 \leq s \leq 1$

$$
\begin{aligned}
s^{\frac{1}{2}} \arctan s^{\frac{1}{2}} & =s^{\frac{1}{2}} \int_{0}^{s^{\frac{1}{2}}} \frac{1}{1+x^{2}} d x \geq s^{\frac{1}{2}} \int_{0}^{s^{\frac{1}{2}}} \frac{1}{1+s} d x \\
& =\frac{s}{1+s} \geq \frac{s}{2}
\end{aligned}
$$

Therefore we have

$$
\int_{B_{1}(0)} \frac{1}{|\xi|^{\frac{\alpha}{2}} \arctan |\xi|^{\frac{\alpha}{2}}} d \xi \leq 2 \int_{B_{1}(0)}|\xi|^{-\alpha} d \xi
$$

Hence $\frac{1}{|\xi|^{\frac{\alpha}{2}} \arctan |\xi|^{\frac{\alpha}{2}}} \in L_{l o c}^{1}\left(\mathbb{R}^{n}\right)$ given that

$$
\begin{aligned}
& n=1: \alpha<1 \\
& n=2: \alpha<2 \\
& n \geq 3: \alpha<n .
\end{aligned}
$$

Moreover $\frac{1}{|\xi|^{\frac{\alpha}{2}} \arctan |\xi|^{\frac{\alpha}{2}}} \in L_{\text {loc }}^{2}\left(\mathbb{R}^{n}\right)$ given that

$$
\begin{aligned}
& n=1: \alpha<\frac{1}{2} \\
& n=2: \alpha<1 \\
& n \geq 3: \alpha<\frac{n}{2}
\end{aligned}
$$

We will now look at similar examples with regard to two variables. In order to discuss this we need to introduce a new notation

$$
B_{1}^{\left(n_{1}, n_{2}\right)}(0)=\left\{(x, y) \in \mathbb{R}^{n_{1}} \times\left.\mathbb{R}^{n_{2}}|| x\right|^{2}+|y|^{2}<1\right\}
$$

for the unit ball in $\mathbb{R}^{n}=\mathbb{R}^{n_{1}+n_{2}}$. Let $\alpha>\beta, 0<\alpha<1$. For $|\xi|^{2}+|\eta|^{2} \leq 1$ the following inequality also holds:

$$
\begin{aligned}
|\xi|^{\alpha}+|\eta|^{\beta} & \geq|\xi|^{\alpha}+|\eta|^{\alpha} \\
& \geq\left(|\xi|^{2}+|\eta|^{2}\right)^{\frac{\alpha}{2}}
\end{aligned}
$$


Example 6.2.4. Consider the continuous negative definite function $\psi(\xi, \eta)=$ $|\xi|^{\alpha}+|\eta|^{\beta}$ for $\alpha>\beta$ and $0<\alpha<1$. Therefore given (6.6) we can write

$$
\begin{aligned}
\int_{B_{1}^{\left(n_{1}, n_{2}\right)}(0)} \frac{1}{|\xi|^{\alpha}+|\eta|^{\beta}} d \xi d \eta & \leq \int_{B_{1}^{\left(n_{1}, n_{2}\right)}(0)}\left(|\xi|^{2}+|\eta|^{2}\right)^{-\frac{\alpha}{2}} d \xi d \eta \\
& =c_{n_{1}+n_{2}} \int_{0}^{1} r^{-\alpha} r^{n_{1}+n_{2}-1} d r \\
& =\left.c_{n_{1}+n_{2}} \frac{1}{n_{1}+n_{2}-\alpha} r^{n_{1}+n_{2}-\alpha}\right|_{0} ^{1} .
\end{aligned}
$$

Therefore $\frac{1}{|\xi|^{\alpha}+|\eta|^{\beta}} \in L_{\text {loc }}^{1}\left(\mathbb{R}^{n}\right)$ given that $\alpha<n_{1}+n_{2}$ which implies $\frac{1}{|\xi|^{\alpha}+|\eta|^{\beta}} \in$ $L_{\text {loc }}^{2}\left(\mathbb{R}^{n}\right)$ if and only if $\alpha<\frac{n_{1}+n_{2}}{2}$. However, for $0<\beta<\alpha<2$ requires cases.

Example 6.2.5. Consider the continuous negative definite function $\psi(\xi, \eta)=$ $\frac{|\xi|^{\alpha}+|\eta|^{\beta}}{1+|\xi|^{\alpha}+|\eta|^{\beta}}$ for $\alpha>\beta$ and $0<\alpha<1$. Then we can write using (6.4) and (6.6)

$$
\begin{aligned}
\int_{B_{1}^{\left(n_{1}, n_{2}\right)}(0)}\left(\frac{|\xi|^{\alpha}+|\eta|^{\beta}}{1+|\xi|^{\alpha}+|\eta|^{\beta}}\right)^{-1} d \xi d \eta & \leq 2 \int_{B_{1}^{\left(n_{1}, n_{2}\right)}(0)} \frac{1}{|\xi|^{\alpha}+|\eta|^{\beta}} d \xi d \eta \\
& \leq 2 \int_{B_{1}^{\left(n_{1}, n_{2}\right)}(0)}\left(|\xi|^{2}+|\eta|^{2}\right)^{-\frac{\alpha}{2}} d \xi d \eta \\
& =2 c_{n_{1}+n_{2}} \int_{0}^{1} r^{-\alpha} r^{n_{1}+n_{2}-1} d r \\
& =\left.2 c_{n_{1}+n_{2}-\alpha} \frac{1}{n_{1}+n_{2}-\alpha} r^{n_{1}+n_{2}-\alpha}\right|_{0} ^{1}
\end{aligned}
$$

Therefore $\left(\frac{|\xi|^{\alpha}+|\eta|^{\beta}}{1+|\xi|^{\alpha}+|\eta|^{\beta}}\right)^{-1} \in L_{l o c}^{1}\left(\mathbb{R}^{n}\right)$ if and only if $\alpha<n_{1}+n_{2}$ which implies $\left(\frac{|\xi|^{\alpha}+|\eta|^{\beta}}{1+|\xi|^{\alpha}+|\eta|^{\beta}}\right)^{-1} \in L_{l o c}^{2}\left(\mathbb{R}^{n}\right)$ if and only if $\alpha<\frac{n_{1}+n_{2}}{2}$.

Example 6.2.6. Consider the continuous negative definite function $\psi(\xi, \eta)=$ $\left(|\xi|^{\alpha}+|\eta|^{\beta}\right)^{\frac{1}{2}} \arctan \left(|\xi|^{\alpha}+|\eta|^{\beta}\right)^{\frac{1}{2}}$ for $\alpha>\beta$ and $0<\alpha<1$. Then we can write using (6.5) and (6.6)

$$
\begin{aligned}
\int_{B_{1}^{\left(n_{1}, n_{2}\right)}(0)} \frac{1}{\left(|\xi|^{\alpha}+|\eta|^{\beta}\right)^{\frac{1}{2}} \arctan \left(|\xi|^{\alpha}+|\eta|^{\beta}\right)^{\frac{1}{2}}} & \leq 2 \int_{B_{1}^{\left(n_{1}, n_{2}\right)}(0)} \frac{1}{|\xi|^{\alpha}+|\eta|^{\beta}} d \xi d \eta \\
& \leq 2 \int_{B_{1}^{\left(n_{1}, n_{2}\right)}(0)}\left(|\xi|^{2}+|\eta|^{2}\right)^{-\frac{\alpha}{2}} d \xi d \eta \\
& =2 c_{n_{1}+n_{2}} \int_{0}^{1} r^{-\alpha} r^{n_{1}+n_{2}-1} d r \\
& =\left.2 c_{n_{1}+n_{2}} \frac{1}{n_{1}+n_{2}-\alpha} r^{n_{1}+n_{2}-\alpha}\right|_{0} ^{1}
\end{aligned}
$$


Therefore $\frac{1}{\left(|\xi|^{\alpha}+|\eta|^{\beta}\right)^{\frac{1}{2}} \arctan \left(|\xi|^{\alpha}+|\eta|^{\beta}\right)^{\frac{1}{2}}} \in L_{l o c}^{1}\left(\mathbb{R}^{n}\right)$ if and only if $\alpha<n_{1}+n_{2}$ which implies $\frac{1}{\left(|\xi|^{\alpha}+|\eta|^{\beta}\right)^{\frac{1}{2}} \arctan \left(|\xi|^{\alpha}+|\eta|^{\beta}\right)^{\frac{1}{2}}} \in L_{l o c}^{2}\left(\mathbb{R}^{n}\right)$ if and only if $\alpha<\frac{n_{1}+n_{2}}{2}$.

We will now discuss examples involving general Bernstein functions.

Example 6.2.7. Consider a Bernstein function $f$, and the continuous negative definite function $\psi(\xi)=|\xi|^{2}$. The function $f(\psi(\xi))=f\left(|\xi|^{2}\right)$ is again a continuous negative definite function. If we assume that

$$
f(s) \geq s^{\rho_{0}}, s<1, \rho_{0}>0
$$

then we have for $n=1$

$$
\begin{aligned}
\int_{-1}^{1} \frac{1}{f\left(|\xi|^{2}\right)} d \xi & \leq \int_{-1}^{1}|\xi|^{-2 \rho_{0}} d \xi=2 \int_{0}^{1} \xi^{-2 \rho_{0}} d \xi \\
& =\left.\frac{2}{1-2 \rho_{0}} \xi^{1-2 \rho_{0}}\right|_{0} ^{1} .
\end{aligned}
$$

Therefore $\frac{1}{f\left(|\xi|^{2}\right)} \in L_{\text {loc }}^{1}\left(\mathbb{R}^{n}\right)$ if and only if $1-2 \rho_{0}>0$, i.e. $\rho_{0}<\frac{1}{2}$ which implies $\frac{1}{f\left(|\xi|^{2}\right)} \in L_{\text {loc }}^{2}\left(\mathbb{R}^{n}\right)$ if and only if $\rho_{0}<\frac{1}{4}$. Moreover for $n=2$ we obtain

$$
\begin{aligned}
\int_{B_{1}(0)} \frac{1}{f\left(|\xi|^{2}\right)} & \leq \int_{B_{1}(0)}|\xi|^{-2 \rho_{0}} d \xi=2 \pi \int_{0}^{1} r^{-2 \rho_{0}} r d r \\
& =2 \pi \int_{0}^{1} r^{1-2 \rho_{0}} d r=\left.2 \pi \frac{1}{2-2 \rho_{0}} r^{2-2 \rho_{0}}\right|_{0} ^{1}
\end{aligned}
$$

Therefore in this case we require $2-2 \rho_{0}>0$, i.e. $\rho_{0}<1$, for local integrability. $\frac{1}{f\left(|\xi|^{2}\right)} \in L_{\text {loc }}^{2}\left(\mathbb{R}^{n}\right)$ if and only if $\rho_{0}<\frac{1}{2}$. Moreover, for higher dimensions, i.e. $n \geq 3$, we have

$$
\begin{aligned}
\int_{B_{1}(0)} \frac{1}{f\left(|\xi|^{2}\right)} & \leq \int_{B_{1}(0)}|\xi|^{-2 \rho_{0}} d \xi=c_{n} \int_{0}^{1} r^{-2 \rho_{0}} r^{n-1} d r \\
& =c_{n} \int_{0}^{1} r^{n-1-2 \rho_{0}} d r=\left.c_{n} \frac{1}{n-2 \rho_{0}} r^{n-2 \rho_{0}}\right|_{0} ^{1}
\end{aligned}
$$

Hence given that $n-2 \rho_{0}>0$, i.e. $\rho_{0}<\frac{n}{2}$ we can conclude that $\frac{1}{f\left(|\xi|^{2}\right)} \in L_{\text {loc }}^{1}\left(\mathbb{R}^{n}\right)$ which implies $\frac{1}{f\left(|\xi|^{2}\right)} \in L_{\text {loc }}^{2}\left(\mathbb{R}^{n}\right)$ if and only if $\rho_{0}<\frac{n}{4}$.

Example 6.2.8. Consider a Bernstein function $f$, and the continuous negative definite function $\psi(\xi, \eta)=|\xi|^{\alpha}+|\eta|^{\beta}$, and the subordinate continuous negative 
definite function $f(\psi(\xi, \eta))=f\left(|\xi|^{2}+|\eta|^{\beta}\right)$. Using (6.6) and (6.7) we obtain

$$
\begin{aligned}
\int_{B_{1}^{\left(n_{1}, n_{2}\right)}(0)} \frac{1}{f\left(|\xi|^{\alpha}+|\eta|^{\beta}\right)} d \xi d \eta & \leq \int_{B_{1}^{\left(n_{1}, n_{2}\right)}(0)} \frac{1}{\left(|\xi|^{\alpha}+|\eta|^{\beta}\right)^{\rho_{0}}} d \xi d \eta \\
& \leq \int_{B_{1}^{\left(n_{1}, n_{2}\right)}(0)}\left(|\xi|^{2}+|\eta|^{2}\right)^{-\frac{\alpha \rho_{0}}{2}} d \xi d \eta \\
& =c_{n_{1}+n_{2}} \int_{0}^{1} r^{-\alpha \rho_{0}} r^{n_{1}+n_{2}-1} d r \\
& =\left.c_{n_{1}+n_{2}} \frac{1}{n_{1}+n_{2}-\alpha \rho_{0}} r^{n_{1}+n_{2}-\alpha \rho_{0}}\right|_{0} ^{1} .
\end{aligned}
$$

Hence $\frac{1}{f\left(|\xi|^{\alpha}+|\eta|^{\beta}\right)} \in L_{\text {loc }}^{1}\left(\mathbb{R}^{n}\right)$ if and only if $n_{1}+n_{2}-\alpha \rho_{0}>0$, i.e. $\rho_{0}<\frac{n_{1}+n_{2}}{\alpha}$ which implies $\frac{1}{f\left(|\xi|^{\alpha}+|\eta|^{\beta}\right)} \in L_{l o c}^{2}\left(\mathbb{R}^{n}\right)$ if and only if $\rho_{0}<\frac{n_{1}+n_{2}}{2 \alpha}$.

For the following example we will discuss a continuous negative definite function introduced in [24].

Example 6.2.9. Consider a continuous negative definite function $\psi_{E R}: \mathbb{R}^{n} \rightarrow$ $\mathbb{R}$

$$
\psi_{E R}(\xi, \eta)=\left(|\xi|^{\alpha_{1}}+|\eta|^{\beta_{1}}\right)^{\gamma_{1}}+\left(|\xi|^{\alpha_{2}}+|\eta|^{\beta 2}\right)^{\gamma_{2}}
$$

for $1<\alpha_{1}, \alpha_{2}<2,1<\beta_{1}, \beta_{2}<2,0<\gamma_{1}, \gamma_{2}<1, \alpha_{1} \gamma_{1}=\beta_{2} \gamma_{2}, \alpha_{2} \gamma_{2}=\beta_{1} \gamma_{1}$, $\alpha_{1} \gamma_{1}>\alpha_{2} \gamma_{2}$ and $\alpha_{i} \gamma_{i}>1, \beta_{i} \gamma_{i}>1$ for $i=1,2$. Then for all $(\xi, \eta) \in \mathbb{R}^{n}$

$$
\kappa_{0}\left(|\xi|^{2}+|\eta|^{2}\right)^{\frac{\alpha_{1} \gamma_{1}}{2}} \leq \psi_{E R}(\xi, \eta)
$$

for some $\kappa_{0}>0$. Therefore we obtain

$$
\begin{aligned}
\int_{B_{1}^{\left(n_{1}, n_{2}\right)}(0)} \frac{1}{\psi_{E R}(\xi, \eta)} & \leq \frac{1}{\kappa_{0}} \int_{B_{1}^{\left(n_{1}, n_{2}\right)}(0)} \frac{1}{\left(|\xi|^{2}+|\eta|^{2}\right)^{\frac{\alpha_{1} \gamma_{1}}{2}}} d \xi d \eta \\
& =\frac{c_{n_{1}+n_{2}}}{\kappa_{0}} \int_{0}^{1} r^{-\alpha_{1} \gamma_{1}} r^{n_{1}+n_{2}-1} d r \\
& =\left.\frac{c_{n_{1}+n_{2}}}{\kappa_{0}} \frac{1}{n_{1}+n_{2}-\alpha_{1} \gamma_{1}} r^{n_{1}+n_{2}-\alpha_{1} \gamma_{1}}\right|_{0} ^{1} .
\end{aligned}
$$

Hence $\frac{1}{\psi_{E R}(\xi, \eta)} \in L_{l o c}^{1}\left(\mathbb{R}^{n}\right)$ if and only if $n_{1}+n_{2}-\alpha_{1} \gamma_{1}>0$, i.e. $\gamma_{1}<\frac{n_{1}+n_{2}}{\alpha_{1}}$. For local integrability in $L^{2}$ we need $n_{1}+n_{2}-2 \alpha_{1} \gamma_{1}>0$. Therefore we can conclude that $\frac{1}{\psi_{E R}(\xi, \eta)} \in L_{l o c}^{2}\left(\mathbb{R}^{n}\right)$ if and only if $\gamma_{1}<\frac{n_{1}+n_{2}}{2 \alpha_{1}}$.

All the examples discussed satisfy the condition of local integrability and therefore by Theorem 4.4 .4 the tempered distribution $E$ defined by $\hat{E}=\frac{1}{\psi}$ is a $H^{\psi, s_{0}}$-fundamental solution. 
Example 6.2.10. In $\mathbb{R}^{2}$ we consider the two convolution semigroups $\left(\mu_{t}^{(1)}\right)_{t \geq 0}$ and $\left(\mu_{t}^{(2)}\right)_{t \geq 0}$ associated with the continuous negative definite functions $\psi^{(1)}(\xi, \eta)=$ $\left(|\xi|^{2}+|\eta|^{2}\right)^{\frac{1}{2}}$ and $\psi^{(2)}(\xi, \eta)=|\xi|+|\eta|$, respectively. The corresponding densities are

$$
p_{t}^{(1)}(x, y)=\frac{1}{2 \pi} \frac{t}{\left(\left(x^{2}+y^{2}\right)+t^{2}\right)^{\frac{3}{2}}}
$$

and

$$
p_{t}^{(2)}(x, y)=\frac{1}{\pi^{2}} \frac{t^{2}}{\left(x^{2}+t^{2}\right)\left(y^{2}+t^{2}\right)}
$$

We note the estimates

$$
\frac{1}{\sqrt{2}} \psi^{(2)}(\xi, \eta) \leq \psi^{(1)}(\xi, \eta) \leq \psi^{(2)}(\xi, \eta)
$$

as well as the different asymptotic behaviour of $p_{t}^{(1)}$ and $p_{t}^{(2)}$, namely for $y=0$ and $x \rightarrow \infty$ we have

$$
p_{1}^{(1)}(x, 0) \sim|x|^{-3}, p_{1}^{(2)}(x, 0) \sim|x|^{-2}
$$

compare with [23], p.1112. Thus, although the symbols $\psi^{(1)}$ and $\psi^{(2)}$ are comparable in the sense that (6.8) holds, the transition densities are not. We now want to study the corresponding potential kernels. Of course $\psi^{(1)}$ is the symbol of the two-dimensional Cauchy process, the corresponding semigroup $\left(T_{t}^{(1)}\right)_{t \geq 0}$ is transient, hence by (6.8) the semigroup $\left(T_{t}^{(2)}\right)_{t \geq 0}$ corresponding to $\psi^{(2)}$ is transient too, and the potential kernel associated with $\left(T_{t}^{(1)}\right)_{t \geq 0}$ is given by

$$
\begin{aligned}
k^{(1)}(x, y) & =\int_{0}^{\infty} p_{t}^{(1)}(x, y) d t \\
& =\frac{1}{2 \pi} \int_{0}^{\infty} \frac{t}{\left(\left(x^{2}+y^{2}\right)+t^{2}\right)^{\frac{3}{2}}} d t=\frac{1}{2 \pi} \frac{1}{\sqrt{x^{2}+y^{2}}} .
\end{aligned}
$$

Note that in this case we have

$$
k^{(1)}(x, y)=\frac{1}{2 \pi} \frac{1}{\psi^{(1)}(x, y)} .
$$

Now we calculate $k^{(2)}(x, y)$ :

$$
k^{(2)}(x, y)=\int_{0}^{\infty} p_{t}^{(2)}(x, y) d t=\frac{1}{\pi^{2}} \int_{0}^{\infty} \frac{t^{2}}{\left(x^{2}+t^{2}\right)\left(y^{2}+t^{2}\right)} d t .
$$


We will use 2.161.3 on p.67 of [12] which gives

$$
\int \frac{t^{2}}{a+b t^{2}+t^{4}} d t=\frac{\sqrt{g}}{\sqrt{c} h} \arctan \frac{\sqrt{c} t}{\sqrt{g}}-\frac{\sqrt{f}}{\sqrt{c} h} \arctan \frac{\sqrt{c} t}{\sqrt{f}}
$$

where for $b^{2}-4 a c>0$

$$
h=\sqrt{b^{2}-4 a c}, \quad f=\frac{b}{2}-\frac{1}{2} h, g=\frac{b}{2}+\frac{1}{2} h .
$$

In our case we have

$$
x^{2} y^{2}+\left(x^{2}+y^{2}\right) t^{2}+t^{4}=a+b t^{2}+c t^{4},
$$

i.e. $c=1, b=x^{2}+y^{2}$ and $a=x^{2} y^{2}$ which implies

$$
\left(x^{2}+y^{2}\right)^{2}-4 x^{2} y^{2}=\left(x^{2}-y^{2}\right)>0 \text { for } x^{2} \neq y^{2}
$$

and for $x \neq y$ we have

$$
\begin{aligned}
& h=\left|x^{2}-y^{2}\right| \\
& f=\frac{x^{2}+y^{2}-\left|x^{2}-y^{2}\right|}{2}=x^{2} \wedge y^{2} \\
& g=\frac{x^{2}+y^{2}+\left|x^{2}-y^{2}\right|}{2}=x^{2} \vee y^{2} .
\end{aligned}
$$

It follows that for $x^{2} \neq y^{2}$

$$
\begin{aligned}
k^{(2)}(x, y) & =\frac{1}{\pi^{2}} \int_{0}^{\infty} \frac{t^{2}}{\left(x^{2}+t^{2}\right)\left(y^{2}+t^{2}\right)} d t \\
& =\frac{1}{2 \pi h}(\sqrt{g}-\sqrt{f}) \\
& =\frac{1}{2 \pi\left|x^{2}-y^{2}\right|}\left(\sqrt{x^{2} \vee y^{2}}-\sqrt{x^{2} \wedge y^{2}}\right) \\
& =\frac{1}{2 \pi\left|x^{2}-y^{2}\right|} \frac{\left(\sqrt{x^{2} \vee y^{2}}-\sqrt{x^{2} \wedge y^{2}}\right)\left(\sqrt{x^{2} \vee y^{2}}+\sqrt{x^{2} \wedge y^{2}}\right)}{\sqrt{x^{2} \vee y^{2}}+\sqrt{x^{2} \wedge y^{2}}}=\frac{1}{\pi} \frac{1}{\sqrt{x^{2} \vee y^{2}}+\sqrt{x^{2} \wedge y^{2}}} \\
& =\frac{\left|x^{2}-y^{2}\right|}{2 \pi\left|x^{2}-y^{2}\right|} \cdot \frac{1}{\sqrt{x^{2} \vee y^{2}}+\sqrt{x^{2} \wedge y^{2}}} \\
& =\frac{1}{2 \pi} \frac{1}{|x|+|y|},
\end{aligned}
$$

and we find eventually

$$
k^{(2)}(x, y)=\frac{1}{2 \pi} \frac{1}{\psi^{(2)}(x, y)},
$$


provided $x^{2} \neq y^{2}$. However, for $x^{2}=y^{2}$ we have

$$
\begin{aligned}
\frac{1}{\pi^{2}} \int_{0}^{\infty} \frac{t^{2}}{\left(x^{2}+t^{2}\right)^{2}} d t & =\left.\frac{1}{\pi^{2}}\left(-\frac{t}{2\left(x^{2}+t^{2}\right)}+\frac{1}{2|x|} \arctan \frac{t}{|x|}\right)\right|_{0} ^{\infty} \\
& =\frac{1}{4 \pi} \frac{1}{|x|}=\frac{1}{2 \pi} \frac{1}{|x|+|x|},
\end{aligned}
$$

and therefore (6.10) holds for all $x, y \in \mathbb{R}$. Note that

$$
k^{(j)}(x, y)=F^{-1}\left(\frac{1}{\psi^{(j)}}\right)(x, y)
$$

and since $n=2$ and both $\psi^{(1)}$ and $\psi^{(2)}$ are homogeneous of degree 1 we expect $k^{(j)}$ to be homogeneous of degree -1 . This example shows that in general the smoothness of $\psi$ gives a limitation of the smoothness of $k$ in the sense that the singular support of $k$ will in general depend on the singular support of $\psi$. In the case of $\psi^{(2)}(\xi, \eta)=|\xi|+|\eta|$ we have

$$
\operatorname{sing} \operatorname{supp} \psi^{(2)}=(\{0\} \times \mathbb{R}) \cup(\mathbb{R} \times\{0\})
$$

and

$$
\operatorname{sing} \operatorname{supp} k^{(2)}=\{(0,0)\} \cup \operatorname{sing} \operatorname{supp} \psi^{(2)} \text {, }
$$

recall that $k^{(2)}=E^{(2)}$ is the unique fundamental solution as considered before and the local $C^{\infty}$-regularity of solutions of the equation

$$
\psi^{(2)}(D) u=f
$$

is given by the condition

$$
\begin{aligned}
\text { sing supp } u & \subset \text { sing supp } E^{(2)}+\text { sing supp } f \\
& =(\{0\} \times \mathbb{R}) \cup(\mathbb{R} \times\{0\})+\text { sing supp } f,
\end{aligned}
$$

provided $u=E^{(2)} * f$.

The formulae (6.9) and (6.10) are in some sense accidental, but not quite. When considering the continuous negative definite function $\psi_{n, \alpha}(\xi)=|\xi|^{\alpha}, 0<\alpha \leq 2$ in $\mathbb{R}^{n}$ and restrict ourselves to the transient cases, i.e. $n>\alpha$, then we have always

$$
k_{n, \alpha}(x)=c_{n, \alpha}|x|^{\alpha-n}
$$


and for $n=2, \alpha=1$ we have $\alpha-n=-1$, i.e.

$$
k_{2,1}(x)=c_{2,1} \frac{1}{\psi_{2,1}(x)}, n=2,
$$

but also for $n=3, \alpha=\frac{3}{2}$ we find with $\alpha-n=\frac{3}{2}$ that

$$
k_{3, \frac{3}{2}}(x)=c_{3, \frac{3}{2}} \frac{1}{\psi_{3, \frac{3}{2}}(x)}, n=3 .
$$

We know that the potential kernel corresponding to

$$
\varphi(\xi, \eta, \zeta)=|\xi|^{\frac{3}{2}}+|\eta|^{\frac{3}{2}}+|\zeta|^{\frac{3}{2}}
$$

must be homogeneous of degree $-\frac{3}{2}$ since it is given by $F^{-1}\left(\frac{1}{\varphi}\right)$, however we could not find an explicit formula. In light of these observations it might be more insightful to write for $k_{(\alpha)}$

$$
k_{n, \alpha}(x)=c_{n, \alpha} \frac{1}{\left(|x|^{\alpha}\right)^{\frac{n}{\alpha}-1}}=c_{n, \alpha} \frac{1}{\left(\psi_{n, \alpha}(x)\right)^{\frac{n}{\alpha}-1}} .
$$

Note that for $n=4$ and $\alpha=2$, i.e. the Gaussian semigroup in $\mathbb{R}^{4}$, this formula gives again

$$
k_{4,2}(x)=c_{4,2} \frac{1}{|x|^{2}}=c_{4,2} \frac{1}{\psi_{4,2}(x)} .
$$

These observations lead to two problems:

$\underline{\text { Problem } 1}$ Find all continuous real-valued negative definite functions $\psi$ corresponding to a transient convolution semigroup with the property that

$$
F^{-1}\left(\frac{1}{\psi}\right)=c_{\psi} \frac{1}{\psi} .
$$

The reader should note that although (6.11) admits the interpretation of an eigenvalue problem, it must be considered in $\mathcal{S}^{\prime}\left(\mathbb{R}^{n}\right) \cap L_{l o c}^{1}\left(\mathbb{R}^{n}\right)$ and it cannot be reduced to the one in $L^{2}\left(\mathbb{R}^{n}\right)$.

$\underline{\text { Problem } 2}$ Find all continuous real-valued negative definite functions $\psi$ corresponding to a transient convolution semigroup with the properties

$$
d_{\psi}(\xi, \eta)=\psi^{\frac{1}{2}}(\xi-\eta) \text { is a metric }
$$

and for the corresponding potential kernel we find

$$
k(x)=g\left(\frac{1}{\psi(x)}\right)
$$


with a strictly increasing continuous function $g$ such that $g(0)=0$.

Note that for continuous negative definite functions solving Problem 2 we can study the local regularity of solutions to the equation $\psi(D) u=f$ by imposing regularity conditions on $f$ in terms of $d_{\psi}$.

In the moment we cannot resolve either of these problems, but some first partial results are available.

We first want to study rotational invariant continuous negative definite functions $\psi: \mathbb{R}^{n} \rightarrow \mathbb{R}$. Each such a function can be written as

$$
\psi(\xi)=f\left(|\xi|^{2}\right)
$$

where $f$ is a Bernstein function, see [35]. For the corresponding potential kernel (in the case that $\left.\frac{1}{\psi} \in L_{l o c}^{1}\left(\mathbb{R}^{n}\right)\right)$ we have

$$
k(x)=F^{-1}\left(\frac{1}{\psi}\right)(x) .
$$

Note that given the subordination $\sqrt{ } \cdot \circ f=f^{\frac{1}{2}}$ which is also a Bernstein function, we have the potential kernel in the case that $\frac{1}{\psi} \in L_{l o c}^{2}\left(\mathbb{R}^{n}\right)$ for $\psi(\xi)=f^{\frac{1}{2}}\left(|\xi|^{2}\right)$. As Fourier transform of a rotational invariant distribution the kernel $k$ must be rotational invariant too, i.e.

$$
k(x)=g\left(|x|^{2}\right)
$$

for a suitable function $g:[0, \infty) \rightarrow \mathbb{R} \cup\{+\infty\}$. Assuming that $f$ has an inverse we can write

$$
k(x)=\left(g \circ f^{-1}\right)\left(f\left(|x|^{2}\right)=\left(\left(g \circ f^{-1}\right) \psi\right)(x) .\right.
$$

Under suitable assumptions on $f$, see [23], we know that

$$
d_{\psi}(\xi, \eta)=\sqrt{f\left(|\xi-\eta|^{2}\right)}
$$

defines a metric on $\mathbb{R}^{n}$ and it follows that

$$
k(x-y)=\left(g \circ f^{-1}\right)\left(d_{\psi}^{2}(x, y)\right)=\left(g \circ f^{-1}\right)\left(|x-y|^{2}\right),
$$

i.e. $k(x)$ is a function of $d_{\psi}^{2}(x, 0)=|x|^{2}$. Moreover, potentials, i.e. functions or distributions of type $k * h$ are given by

$$
(k * u)(x)=\int\left(g \circ f^{-1}\right)\left(d_{\psi}^{2}(x, y)\right) u(y) d y
$$


and their properties, given $u$, depend on $d_{\psi}$. Note that we can deduce more properties of $k(x)=g\left(|x|^{2}\right)$ by using the representation

$$
k(x)=\int_{0}^{\infty} p_{t}^{\psi}(x) d t
$$

where $p_{t}^{\psi}(\cdot)$ is the density corresponding to the convolution semigroup $\left(\mu_{t}^{\psi}\right)_{t \geq 0}$, which we assume to exist, e.g. we may assume $e^{-f\left(|\cdot|^{2}\right)} \in L^{1}\left(\mathbb{R}^{n}\right)$. In our case $p_{t}^{\psi}$ is given by

$$
p_{t}^{\psi}(x)=\int_{0}^{\infty} \frac{1}{(4 \pi s)^{\frac{n}{2}}} e^{-\frac{|x|^{2}}{4 s}} \eta_{t}(d s)
$$

where $\left(\eta_{t}\right)_{t \geq 0}$ is the convolution semigroup supported on $[0, \infty)$ corresponding to $f$. Since the Gauss kernel is strictly positive, $p_{t}^{\psi}$ is strictly positive on $\mathbb{R}^{n}$ and this implies already that $\left(T_{t}^{\psi}\right)_{t \geq 0}$ is irreducible. Furthermore we have for $t>0$

$$
\left\|p_{t}^{\psi}(\cdot)\right\|_{\infty}=\int_{0}^{\infty} \frac{1}{(4 \pi s)^{\frac{n}{2}}} \eta_{t}(d s)=p_{t}(0) .
$$

Clearly $p_{t}^{\psi}(x)$ is rotational invariant and if we assume that $r \mapsto \tilde{p}_{t}^{\psi}(r)=p_{t}^{\psi}(x)$, $r=|x|$, is strictly decreasing in $r$ for $t$ fixed it follows that $r \mapsto g\left(r^{2}\right)$ is strictly decreasing, hence $g \circ f^{-1}$ is strictly decreasing. In summary, this example shows that interpreting $\psi$ as a square of a metric gives the possibility to interpret both, $p_{t}$ and $k$, as a function of this metric, i.e. it allows a geometric interpretation of $p_{t}$ and $k$.

Let $f$ be a Bernstein function such that $f(0)=0$ and $(\xi, \eta) \mapsto \frac{1}{f(|\xi|+|\eta|)}$ is an element in $L_{l o c}^{1}\left(\mathbb{R}^{2}\right)$. Hence the corresponding semigroup is transient and $F^{-1}\left(\frac{1}{f(|\xi|+|\eta|)}\right)(x, y)$ is the potential kernel, thus fundamental solution to $\psi(D)$, $\psi(\xi, \eta)=f(|\xi|+|\eta|)$. We have to find the integral

$$
2 \pi F^{-1}\left(\frac{1}{f(|\xi|+|\eta|)}\right)(x, y)=\int_{\mathbb{R}^{2}} e^{i x \xi} e^{i y \eta} \frac{1}{f(|\xi|+|\eta|)} d(\xi, \eta) .
$$

First we consider with $\mathbb{R}_{+}=(0, \infty)$

$$
\int_{\left(\mathbb{R}_{+}\right)^{2}} e^{i x \xi} e^{i y \eta} \frac{1}{f(|\xi|+|\eta|)} d(\xi, \eta)
$$

and introduce the new coordinates

$$
s=\xi+\eta, r=\xi-\eta
$$


or

$$
\xi=\frac{s+r}{2}, \eta=\frac{s-r}{2} .
$$

The set $\left(\mathbb{R}_{+}\right)^{2}$ is mapped onto the set $\left\{(r, s) \in \mathbb{R}^{2} \mid s>0\right.$ and $\left.|r|<s\right\}$ and by the transformation theorem we find

$$
\begin{aligned}
\int_{\left(\mathbb{R}_{+}\right)^{2}} e^{i x \xi} e^{i y \eta} \frac{1}{f(|\xi|+|\eta|)} d(\xi, \eta) & =\int_{\left(\mathbb{R}_{+}\right)^{2}} e^{i x \xi} e^{i y \eta} \frac{1}{f(\xi+\eta)} d(\xi, \eta) \\
& =\frac{1}{2} \int_{0}^{\infty} e^{i\left(\frac{x+y}{2}\right) s} \frac{1}{f(s)}\left(\int_{-s}^{s} e^{i\left(\frac{x-y}{2}\right) r} d r\right) d s .
\end{aligned}
$$

We note that

$$
\int_{-s}^{s} e^{i\left(\frac{x-y}{2}\right) r} d r=\int_{-s}^{s} \cos \left(\frac{x-y}{2}\right) r d r=2 \frac{1}{\left(\frac{x-y}{2}\right)} \sin \left(\frac{x-y}{2}\right) s
$$

which yields

$$
\int_{\left(\mathbb{R}_{+}\right)^{2}} e^{i x \xi} e^{i y \eta} \frac{1}{f(\xi+\eta)} d(\xi, \eta)=\frac{1}{\frac{(x-y)}{2}} \int_{0}^{\infty} e^{i\left(\frac{x+y}{2}\right) s} \frac{1}{f(s)} \sin \left(\frac{x-y}{2}\right) s d s .
$$

Since $e^{i\left(\frac{x+y}{2}\right) s} \sin \left(\frac{x-y}{2}\right) s=\frac{1}{2}(\sin x s-\sin y s)+\frac{i}{2}(\cos y s-\cos x s)$ it follows that

$$
\int_{\left(\mathbb{R}_{+}\right)^{2}} e^{i x \xi} e^{i y \eta} \frac{1}{f(\xi+\eta)} d(\xi, \eta)=\frac{1}{2\left(\frac{x-y}{2}\right)} \int_{0}^{\infty}(\sin x s-\sin y s+i(\cos y s-\cos x s)) \frac{1}{f(s)} d s .
$$

Now we return to (6.12) and note that the integral on the right hand side is given by

$$
\begin{aligned}
& \int_{\mathbb{R}^{2}} e^{i x \xi} e^{i y \eta} \frac{1}{f(|\xi|+|\eta|)} d(\xi, \eta) \\
& =\left(\int_{\left(\mathbb{R}_{+}\right)^{2}}+\int_{\mathbb{R}_{+} \times \mathbb{R}_{-}}+\int_{\mathbb{R}_{-} \times \mathbb{R}_{+}}+\int_{\left(\mathbb{R}_{-}\right)^{2}}\right)\left(e^{i x \xi} e^{i y \eta} \frac{1}{f(|\xi|+|\eta|)}\right) d(\xi, \eta) \\
& =I_{1}+I_{2}+I_{3}+I_{4}
\end{aligned}
$$

with

$$
\begin{gathered}
I_{1}=\frac{1}{2\left(\frac{x-y}{2}\right)} \int_{0}^{\infty}(\sin x s-\sin y s+i(\cos y s-\cos x s)) \frac{1}{f(s)} d s, \\
I_{2}=\frac{1}{2\left(\frac{x+y}{2}\right)} \int_{0}^{\infty}(\sin x s+\sin y s+i(\cos y s-\cos x s)) \frac{1}{f(s)} d s, \\
I_{3}=\frac{1}{2\left(\frac{-x-y}{2}\right)} \int_{0}^{\infty}(-\sin x s-\sin y s+i(\cos y s-\cos x s)) \frac{1}{f(s)} d s
\end{gathered}
$$


and

$$
I_{4}=\frac{1}{2\left(\frac{-x+y}{2}\right)} \int_{0}^{\infty}(-\sin x s+\sin y s+i(\cos y s-\cos x s)) \frac{1}{f(s)} d s
$$

Adding (6.13)-(6.16) we arrive at

$$
\begin{aligned}
& \int_{\mathbb{R}^{2}} e^{i x \xi} e^{i y \eta} \frac{1}{f(|\xi|+|\eta|)} d(\xi, \eta) \\
& =\frac{2}{x-y} \int_{0}^{\infty}(\sin x s-\sin y s) \frac{1}{f(s)} d s+\frac{2}{x+y} \int_{0}^{\infty}(\sin x s+\sin y s) \frac{1}{f(s)} d s
\end{aligned}
$$

or

$$
2 \pi F^{-1}\left(\frac{1}{f(|\xi|+|\eta|)}\right)(x, y)=\frac{4}{x^{2}-y^{2}} \int_{0}^{\infty}(x \sin x s-y \sin y s) \frac{1}{f(s)} d s .
$$

Thus, in order to find the potential kernel associated with $f(|\xi|+|\eta|)$ we need to evaluate the integral

$$
\int_{0}^{\infty} \frac{\sin a s}{f(s)} d s, a>0 .
$$

Note that it is sometimes helpful to write the right hand side of (6.17) as

$$
\frac{4}{|x|^{2}-|y|^{2}} \int_{0}^{\infty}(|x| \sin |x| s-|y| \sin |y| s) \frac{1}{f(s)} d s .
$$

We want to apply (6.18) to $f(s)=s^{\alpha}, 0<\alpha<1$. For this we note, compare [12], 3.7614, p.420, that

$$
\int_{0}^{\infty} \frac{\sin a s}{s^{\alpha}} d s=\Gamma(1-\alpha) \sin \left(\frac{1-\alpha}{2} \pi\right) \frac{1}{a^{1-\alpha}} .
$$

Now we obtain

Example 6.2.11. The potential kernel $k$ corresponding to $\psi(\xi, \eta)=(|\xi|+|\eta|)^{\alpha}$, $0<\alpha<1$, is given by

$$
\begin{aligned}
k(x, y) & =F^{-1}\left(\frac{1}{(|\xi|+|\eta|)^{\alpha}}\right)(x, y)=\frac{2}{\pi} \frac{1}{|x|^{2}-|y|^{2}} \int_{0}^{\infty}(|x| \sin |x| s-|y| \sin |y| s) \frac{1}{s^{\alpha}} d s \\
& =\frac{2}{\pi} \Gamma(1-\alpha) \sin \left(\frac{1-\alpha}{2} \pi\right) \frac{1}{|x|^{2}-|y|^{2}}\left(\frac{|x|}{|x|^{1-\alpha}}-\frac{|y|}{|y|^{1-\alpha}}\right) \\
& =\frac{2}{\pi} \Gamma(1-\alpha) \sin \left(\frac{1-\alpha}{2} \pi\right) \frac{1}{|x|^{2}-|y|^{2}}\left(|x|^{\alpha}-|y|^{\alpha}\right) \\
& =\frac{2}{\pi} \Gamma(1-\alpha) \sin \left(\frac{1-\alpha}{2} \pi\right) \frac{1}{|x|+|y|}\left(\frac{|x|^{\alpha}-|y|^{\alpha}}{|x|-|y|}\right),|x| \neq|y| .
\end{aligned}
$$


We note that $k$ is homogeneous of degree $-2+\alpha$ and since $\frac{1}{(|\xi|+|\eta|)^{\alpha}}$ is homogeneous of degree $-\alpha$, this is exactly what we expect. Moreover, $|x|>|y|$ implies $|x|^{\alpha}>|y|^{\alpha}$ and therefore $k(x, y) \geq 0$, in fact $k(x, y)>0$ for $|x| \neq|y|$. Since for $t>0$ we have

$$
\frac{d}{d t} t^{\alpha}=\alpha t^{\alpha-1}=\lim _{s \rightarrow t} \frac{t^{\alpha}-s^{\alpha}}{t-s}
$$

we find for $|x|,|y|>0$ that

$$
\lim _{|y| \rightarrow|x|} \frac{1}{|x|+|y|}\left(\frac{|x|^{\alpha}-|y|^{\alpha}}{|x|-|y|}\right)=\frac{\alpha}{2}|x|^{-1}|x|^{\alpha-1}=\frac{\alpha}{2}|x|^{\alpha-2},
$$

and therefore we define

$$
k(x, x):=\frac{\alpha}{\pi} \Gamma(1-\alpha) \sin \left(\frac{1-\alpha}{2} \pi\right) \frac{1}{|x|^{2-\alpha}} .
$$

For general $0<\alpha<1$ we do not see a simple way to relate $k(x, y)$ in Example 6.2.11 to $\psi(\xi, \eta)=(|\xi|+|\eta|)^{\alpha}$. However, there are some interesting special cases worth discussing:

1. For $\alpha=\frac{1}{2}$ we have $\psi(\xi, \eta)=(|\xi|+|\eta|)^{\frac{1}{2}}$ and

$$
\begin{aligned}
k_{\frac{1}{2}}(x, y) & =\frac{2}{\pi} \Gamma\left(\frac{1}{2}\right) \sin \left(\frac{\pi}{4}\right) \frac{1}{|x|+|y|}\left(\frac{|x|^{\frac{1}{2}}-|y|^{\frac{1}{2}}}{|x|-|y|}\right) \\
& =\sqrt{\frac{2}{\pi}} \frac{1}{|x|+|y|} \frac{|x|^{\frac{1}{2}}-|y|^{\frac{1}{2}}}{\left(|x|^{\frac{1}{2}}-|y|^{\frac{1}{2}}\right)\left(|x|^{\frac{1}{2}}+|y|^{\frac{1}{2}}\right)} \\
& =\sqrt{\frac{2}{\pi}} \frac{1}{(|x|+|y|)\left(|x|^{\frac{1}{2}}+|y|^{\frac{1}{2}}\right)} .
\end{aligned}
$$

Thus we have the exact formula

$$
k_{\frac{1}{2}}(x, y)=\sqrt{\frac{2}{\pi}} \frac{1}{(|x|+|y|)\left(|x|^{\frac{1}{2}}+|y|^{\frac{1}{2}}\right)} .
$$

Since

$$
\frac{1}{2}\left(|x|^{\frac{1}{2}}+|y|^{\frac{1}{2}}\right)^{2} \leq|x|+|y| \leq\left(|x|^{\frac{1}{2}}+|y|^{\frac{1}{2}}\right)^{2}
$$

or

$$
\frac{1}{\left(|x|^{\frac{1}{2}}+|y|^{\frac{1}{2}}\right)^{2}} \leq \frac{1}{|x|+|y|} \leq \frac{2}{\left(|x|^{\frac{1}{2}}+|y|^{\frac{1}{2}}\right)^{2}}
$$

we find also the two estimates

$$
\sqrt{\frac{2}{\pi}} \frac{1}{\left(|x|^{\frac{1}{2}}+|y|^{\frac{1}{2}}\right)^{3}} \leq k_{\frac{1}{2}}(x, y) \leq \frac{2 \sqrt{2}}{\sqrt{\pi}} \frac{1}{\left(|x|^{\frac{1}{2}}+|y|^{\frac{1}{2}}\right)^{3}}
$$


and

$$
\frac{1}{\sqrt{2 \pi}} \frac{1}{(|x|+|y|)^{\frac{3}{2}}} \leq k_{\frac{1}{2}}(x, y) \leq \sqrt{\frac{2}{\pi}} \frac{1}{(|x|+|y|)^{\frac{3}{2}}} .
$$

The latter estimates we can write however as

$$
\frac{1}{\sqrt{2 \pi}} \frac{1}{\psi(x, y)^{3}} \leq k_{\frac{1}{2}}(x, y) \leq \sqrt{\frac{2}{\pi}} \frac{1}{\psi(x, y)^{3}},
$$

or with $d_{\psi}\left(\left(x_{1}, y_{1}\right),\left(x_{2}, y_{2}\right)\right)=\psi^{\frac{1}{2}}\left(x_{1}-x_{2}, y_{1}-y_{2}\right)$ we obtain

$$
\frac{1}{\sqrt{2 \pi}} \frac{1}{d_{\psi}^{6}\left(\left(x_{1}, y_{1}\right),\left(x_{2}, y_{2}\right)\right)} \leq k_{\frac{1}{2}}\left(x_{1}-x_{2}, y_{1}-y_{2}\right) \leq \sqrt{\frac{2}{\pi}} \frac{1}{d_{\psi}^{6}\left(\left(x_{1}, y_{1}\right),\left(x_{2}, y_{2}\right)\right)}
$$

2. For $\alpha=\frac{1}{4}$, i.e. $\psi(\xi, \eta)=(|\xi|+|\eta|)^{\frac{1}{4}}$, we find

$$
\begin{aligned}
k_{\frac{1}{4}}(x, y) & =\frac{2}{\pi} \Gamma\left(\frac{3}{4}\right) \sin \frac{3}{8} \pi \frac{1}{|x|+|y|} \frac{|x|^{\frac{1}{4}}-|y|^{\frac{1}{4}}}{|x|-|y|} \\
& =\frac{\sqrt{2+\sqrt{2}}}{\pi} \Gamma\left(\frac{3}{4}\right) \frac{1}{|x|+|y|} \frac{|x|^{\frac{1}{4}}-|y|^{\frac{1}{4}}}{\left(|x|^{\frac{1}{2}}+|y|^{\frac{1}{2}}\right)\left(|x|^{\frac{1}{4}}+|y|^{\frac{1}{4}}\right)\left(|x|^{\frac{1}{4}}-|y|^{\frac{1}{4}}\right)} \\
& =\frac{\sqrt{2+\sqrt{2}}}{\pi} \Gamma\left(\frac{3}{4}\right) \frac{1}{(|x|+|y|)\left(|x|^{\frac{1}{2}}+|y|^{\frac{1}{2}}\right)\left(|x|^{\frac{1}{4}}-|y|^{\frac{1}{4}}\right)}
\end{aligned}
$$

and now we look at $\alpha=\frac{1}{2^{m}}, m \in \mathbb{N}$. We note that

$$
|x|-|y|=\left(\prod_{l=1}^{m}\left(|x|^{\frac{1}{2} l}+|y|^{\frac{1}{2} l}\right)\right)\left(|x|^{\frac{1}{2} m}-|y|^{\frac{1}{2} m}\right)
$$

which follows by induction and therefore we get

$$
\frac{|x|^{\frac{1}{2} m}-|y|^{\frac{1}{2} m}}{|x|-|y|}=\frac{1}{\prod_{l=1}^{m}\left(|x|^{\frac{1}{2} l}+|y|^{\frac{1}{2} l}\right)}
$$

and

$$
k_{\frac{1}{2^{m}}}(x, y)=\frac{2}{\pi} \Gamma\left(1-\frac{1}{2^{m}}\right) \sin \left(\left(\frac{1-2^{-m}}{2}\right) \pi\right)\left(\frac{1}{|x|+|y|}\right) \frac{1}{\prod_{l=1}^{m}\left(|x|^{\frac{1}{2} l}+|y|^{\frac{1}{2} l}\right)}
$$

or

$$
k_{\frac{1}{2^{m}}}(x, y)=\frac{2}{\pi} \Gamma\left(1-\frac{1}{2^{m}}\right) \sin \left(\left(\frac{1-2^{-m}}{2}\right) \pi\right) \frac{1}{\prod_{l=0}^{m}\left(|x|^{\frac{1}{2} l}+|y|^{\frac{1}{2} l}\right)} .
$$

With the help of (6.19) we deduce the existence of two constants $0<A_{l}^{(1)}<A_{l}^{(2)}$ such that

$$
A_{l}^{(1)} \frac{1}{\left(|x|^{\frac{1}{2} l}+|y|^{\frac{1}{2} l}\right)} \leq \frac{1}{(|x|+|y|)^{\frac{1}{2} l}} \leq A_{l}^{(2)} \frac{1}{\left(|x|^{\frac{1}{2} l}+|y|^{\frac{1}{2} l}\right)}
$$


implying

$$
\prod_{l=0}^{m} A_{l}^{(1)} \frac{1}{\left(|x|^{\frac{1}{2} l}+|y|^{\frac{1}{2} l}\right)} \leq \prod_{l=0}^{m} \frac{1}{(|x|+|y|)^{\frac{1}{2} l}} \leq \prod_{l=0}^{m} A_{l}^{(2)} \frac{1}{\left(|x|^{\frac{1}{2} l}+|y|^{\frac{1}{2} l}\right)},
$$

and with

$$
\begin{gathered}
\kappa_{m}:=\sum_{l=0}^{m} \frac{1}{2^{l}}=2-\frac{1}{2^{m}} \\
\sigma_{m}:=\prod_{l=0}^{m} A_{l}^{(1)}, \quad \tau_{m}=\prod_{l=0}^{m} A_{l}^{(2)}
\end{gathered}
$$

we have

$$
\sigma_{m} \prod_{l=0}^{m} \frac{1}{\left(|x|^{\frac{1}{2} l}+|y|^{\frac{1}{2} l}\right)} \leq \frac{1}{(|x|+|y|)^{\kappa_{m}}} \leq \tau_{m} \prod_{l=0}^{m} \frac{1}{\left(|x|^{\frac{1}{2} l}+|y|^{\frac{1}{2} l}\right)} .
$$

Eventually we arrive at

$$
\tilde{\sigma}_{m} \frac{1}{(|x|+|y|)^{\kappa_{m}}} \leq k_{\frac{1}{2^{m}}}(x, y) \leq \tilde{\tau}_{m} \frac{1}{(|x|+|y|)^{\kappa_{m}}}
$$

i.e.

$$
\sigma_{m}^{*} \frac{1}{\psi(x, y)^{2^{m} \kappa_{m}}} \leq k_{\frac{1}{2^{m}}}(x, y) \leq \tau_{m}^{*} \frac{1}{\psi(x, y)^{2^{m} \kappa_{m}}}
$$

or

$$
\sigma_{m}^{*} \frac{1}{d_{\psi}^{2\left(2^{m+1}-1\right)}((x, y),(0,0))} \leq k_{\frac{1}{2^{m}}}(x, y) \leq \tau_{m}^{*} \frac{1}{d_{\psi}^{2\left(2^{m+1}-1\right)}((x, y),(0,0))}
$$

which are for $m=1$ the estimates (6.20). Moreover $\frac{1}{d_{\psi}^{2\left(2^{m+1}-1\right)}((x, y),(0,0))}$ is homogeneous of degree $\left(-2^{m+1}+1\right) \frac{1}{2^{m}}=-2+\frac{1}{2^{m}}$, as we expect.

So far we do not have a similar result for the case of a general $\alpha \in(0,1)$.

\subsection{Interpretations and Generalization}

In section 6.1 we have outlined some properties of the potential kernel (of a transient convolution semigroup). The examples in section 6.2 indicate that if the continuous negative definite function $\psi: \mathbb{R}^{n} \rightarrow \mathbb{R}$ gives rise to a metric, the potential kernel is either a function of this metric, or it admits (two-sided) controls by this metric. We have formulated this observation in two problems, 
Problem 1 and Problem 2, in section 6.2. In the moment we are far away to provide for these problems a general solution. However, the positive examples are motivation enough to explore corresponding ideas and this section is devoted to some consequences in cases where the problems admit a solution as desired.

First let us assume that the potential kernel $k$ is given

$$
k(x-y)=g\left(\frac{1}{\psi(x-y)}\right)=h\left(\frac{1}{d_{\psi}(x, y)}\right)
$$

where $h(r)=g\left(r^{2}\right)$. Assuming that $k$ gives a fundamental solution to $\psi(D)$, a solution to $\psi(D) u=v$ is given by

$$
u=E * v=k * v
$$

or (at least formally)

$$
u(x)=\int_{\mathbb{R}^{n}} h\left(\frac{1}{d_{\psi}(x, y)}\right) v(y) d y=\int_{\mathbb{R}^{n}} h\left(\frac{1}{d_{\psi}(y, 0)}\right) v(x-y) d y .
$$

We expect $h$ to have a singularity at 0 , in fact by Lemma 6.1 .2 we must have $h(0)=+\infty$. From (6.23) we deduce for $\eta \in \mathbb{R}^{n}$ that

$$
u(x)-u(x+\eta)=\int_{\mathbb{R}^{n}} h\left(\frac{1}{d_{\psi}(x, z)}\right)(v(z)-v(z+\eta)) d z .
$$

So far we have not stated proper assumptions on $v$, we only implicitly have assumed that (6.22) or (6.23) makes sense. Starting with (6.24) we now can try to derive conditions on $v$ and on $h$ in order to deduce regularity properties of (6.24). For this we make the standard classical approach and split the integral in $(6.24)$ :

$$
\begin{aligned}
& \int_{\mathbb{R}^{n}} h\left(\frac{1}{d_{\psi}(x, z)}\right)(v(z)-v(z+\eta)) d z \\
& =\int_{d_{\psi}(x, z)<r} h\left(\frac{1}{d_{\psi}(x, z)}\right)(v(z)-v(z+\eta)) d z \\
& +\int_{d_{\psi}(x, z) \geq r} h\left(\frac{1}{d_{\psi}(x, z)}\right)(v(z)-v(z+\eta)) d z .
\end{aligned}
$$

Let us consider the first integral in (6.25), i.e.

$$
\int_{d_{\psi}(x, z)<r} h\left(\frac{1}{d_{\psi}(x, z)}\right)(v(z)-v(z+\eta)) d z .
$$


Suppose we can find a non-negative continuous function $\omega: \mathbb{R} \rightarrow \mathbb{R}$ such that $\omega(0)=0, \omega(t)>0$ for $t \neq 0$, and for some $\alpha>0$

$$
\omega(\delta)^{\alpha} \int_{\frac{\delta}{2} \leq d_{\psi}(z, 0)<r} h\left(\frac{1}{d_{\psi}(z, 0)}\right) d z \leq M(r)<\infty
$$

for all $\delta \leq r$. We now consider bounded functions $v: \mathbb{R}^{n} \rightarrow \mathbb{R}$ such that

$$
|v(x)-v(y)| \leq\left(\omega\left(d_{\psi}(x, y)\right)^{\beta}, \quad \alpha<\beta\right.
$$

Let $0<\gamma<\beta-\alpha$ and $0<\delta<r$. It follows with the help of (6.26) that

$$
\begin{aligned}
& \left|\frac{1}{\omega\left(d_{\psi}(x, x+\eta)\right)^{\gamma}} \int_{\frac{\delta}{2} \leq d_{\psi}(x, z)<r} h\left(\frac{1}{d_{\psi}(x, z)}\right)(v(z)-v(z+\eta)) d z\right| \\
& =\left|\frac{1}{\omega\left(d_{\psi}(\eta, 0)\right)^{\gamma}} \int_{\frac{\delta}{2} \leq d_{\psi}(z, 0)<r} h\left(\frac{1}{d_{\psi}(z, 0)}\right)(v(x-z)-v(x-z+\eta)) d z\right| \\
& \leq \frac{1}{\omega\left(d_{\psi}(\eta, 0)\right)^{\gamma}} \int_{\frac{\delta}{2} \leq d_{\psi}(z, 0)<r} h\left(\frac{1}{d_{\psi}(z, 0)}\right)|v(x-z)-v(x-z+\eta)| d z \\
& \leq \frac{1}{\omega\left(d_{\psi}(\eta, 0)\right)^{\gamma}} \int_{\frac{\delta}{2} \leq d_{\psi}(z, 0)<r} h\left(\frac{1}{d_{\psi}(z, 0)}\right)\left(\omega\left(d_{\psi}(\eta, 0)\right)^{\beta} d z\right. \\
& =\left(\int_{\frac{\delta}{2} \leq d_{\psi}(z, 0)<r} h\left(\frac{1}{d_{\psi}(z, 0)}\right) d z\left(\omega\left(d_{\psi}(\eta, 0)\right)\right)^{\alpha}\right)\left(\omega\left(d_{\psi}(\eta, 0)\right)\right)^{\beta-\alpha-\gamma} .
\end{aligned}
$$

The continuity of $\omega$ implies that for $\varepsilon>0$ there exists $\delta>0$ such that $d_{\psi}(\eta, 0)<$ $\delta$ implies $\omega\left(d_{\psi}(\eta, 0)\right)<\varepsilon^{\frac{1}{\beta-\alpha-\gamma}}$ which implies

$$
\begin{aligned}
& \left|\frac{1}{\left(\omega\left(d_{\psi}(x, x+\eta)\right)\right)^{\gamma}} \int_{\frac{\delta}{2} \leq d_{\psi}(x, z)<r} h\left(\frac{1}{d_{\psi}(x, z)}\right)(v(z)-v(z+\eta)) d z\right| \\
& \leq\left(\left((\omega(\delta))^{\alpha} \int_{\frac{\delta}{2} \leq d_{\psi}(z, 0)<r} h\left(\frac{1}{d_{\psi}(z, 0)}\right) d z\right)\left(\omega\left(d_{\psi}(\eta, 0)\right)\right)^{\beta-\alpha-\gamma}\right. \\
& \leq M(r) \varepsilon,
\end{aligned}
$$

provided $|v(x)-v(x+\eta)| \leq\left(\omega\left(d_{\psi}(\eta, 0)\right)\right)^{\beta} \leq(\omega(\delta))^{\beta}$, which we can achieve if we add to $\omega$ the condition to be increasing on $[0, R]$ for some $R>0$. 
Now we turn to the second integral in (6.25), i.e. to

$$
\begin{aligned}
& \int_{d_{\psi}(x, z) \geq r} h\left(\frac{1}{d_{\psi}(x, z)}\right)(v(z)-v(z+\eta)) d z \\
& =\int_{d_{\psi}(x, z) \geq r} h\left(\frac{1}{d_{\psi}(x, z)}\right) v(z) d z-\int_{d_{\psi}(x, z) \geq r} h\left(\frac{1}{d_{\psi}(x, z)}\right) v(z+\eta) d z \\
& =\int_{d_{\psi}(y, 0) \geq r} h\left(\frac{1}{d_{\psi}(y, 0)}\right) v(x-y) d y-\int_{d_{\psi}(y, 0) \geq r} h\left(\frac{1}{d_{\psi}(y, 0)}\right) v(x+\eta-y) d y .
\end{aligned}
$$

If $v \in C_{b}\left(\mathbb{R}^{n}\right)$ and $y \mapsto h\left(\frac{1}{d_{\psi}(y, 0)}\right) \chi_{\mathbb{R}^{n} \backslash B_{r}^{d_{\psi}}(0)}(y)$ belongs to $L^{1}\left(\mathbb{R}^{n} \backslash B_{r}^{d_{\psi}}(0)\right)$ then the first integral in (6.28) is a continuous function with respect to $x$ and the second integral in (6.28) is a continuous function with respect to $x$ and $\eta$, compare with Theorem 8.1 in [22].

Thus we have proved

Theorem 6.3.1. Let $k(x)=h\left(\frac{1}{d_{\psi}(x, 0)}\right)$ be the potential kernel corresponding to the transient semigroup $\left(T_{t}^{\psi}\right)_{t \geq 0}$ such that $k$ is also a fundamental solution to $\psi(D)$. Assume for some increasing continuous function $w:[0, \infty) \rightarrow \mathbb{R}$ such that $w(0)=0$ and $w(s)>0$ for $s>0$ that (6.26) holds and that for $r>0$ the function $y \mapsto h\left(\frac{1}{d_{\psi}(y, 0)}\right) \chi_{\mathbb{R}^{n} \backslash B_{r}^{d_{\psi}}(0)}(y)$ belongs to $L^{1}\left(\mathbb{R}^{n} \backslash B_{r}^{d_{\psi}}(0)\right)$. If $v \in C_{b}\left(\mathbb{R}^{n}\right)$ satisfies (6.27) then the function $u: \mathbb{R}^{n} \rightarrow \mathbb{R}, u(x):=(k * v)(x)$ is continuous on $\mathbb{R}^{n}$ and for $x$ in a compact set $K \subset \mathbb{R}^{n}$ we have for every $0<\gamma<\beta-\alpha$

$$
\frac{u(x)-u(x+\eta)}{\left(\omega\left(d_{\psi}(\eta, 0)\right)\right)^{\gamma}} \rightarrow 0 \text { as } \omega\left(d_{\psi}(\eta, 0)\right) \rightarrow 0 \text {. }
$$

Remark 6.3.2. A. Note that (6.29) is a type of local Hölder continuity of $u$ with respect to $d_{\psi}$

B. Our derivation of Theorem 6.3.1 follows of course the ideas of the standard proof given in classical potential theory for the Riesz potentials, see [28].

To verify the conditions of Theorem 6.3.1 requires some knowledge of $k(\cdot)$. For rotational invariant symbols $\psi(\xi)$ we can check these conditions in the following way. In this case the metric $d_{\psi}$ is given by

$$
d_{\psi}(x, y)=d_{\psi}(x-y, 0)=\left(f\left(|x-y|^{2}\right)\right)^{\frac{1}{2}}
$$


for some Bernstein function $f$ and we have

$$
k(x)=h\left(\frac{1}{\left(f\left(|x|^{2}\right)\right)^{\frac{1}{2}}}\right)=\tilde{h}\left(\frac{1}{|x|}\right)
$$

for a suitable function $\tilde{h}$. Once we know that $\tilde{h}$ satisfies the two estimates

$$
c_{R} \rho^{-\kappa-n+1} \leq \tilde{h}\left(\frac{1}{\rho}\right), 0<\kappa<1,0<\rho \leq R
$$

and

$$
\tilde{h}\left(\frac{1}{\rho}\right) \leq \tilde{c}_{R} \rho^{-n+\varepsilon}, \rho \geq R, \varepsilon>0,
$$

we can apply Theorem 6.3.1.

However, if we replace in (6.30) and (6.31) the function $\tilde{h}$ by $h\left(\frac{1}{d_{\psi}(x, 0)}\right), \rho=|x|$, we still can apply Theorem 6.3.1. This is possible in the case of Example 6.2.11 for $k_{\frac{1}{2}}$, i.e.

$$
k_{\frac{1}{2}}(x, y)=\sqrt{\frac{2}{\pi}} \frac{1}{(|x|+|y|)\left(|x|^{2}+|y|^{2}\right)^{\frac{1}{2}}},
$$

which is the potential kernel associated with $\psi: \mathbb{R}^{2} \rightarrow \mathbb{R}, \psi(\xi, \eta)=(|\xi|+|\eta|)^{\frac{1}{2}}$. We have now the estimates (6.20) and with $n=2, \kappa=\frac{1}{2}$ and $\varepsilon=\frac{1}{2}$ we obtain (6.30) and (6.31).

Similarly, we can state the same for $k_{\frac{1}{2^{m}}}$, i.e.

$$
k_{\frac{1}{2^{m}}}(x, y)=\frac{2}{\pi} \Gamma\left(1-\frac{1}{2^{m}}\right) \sin \left(\left(\frac{1-2^{-m}}{2}\right) \pi\right) \frac{1}{\prod_{l=0}^{m}\left(|x|^{\frac{1}{2} l}+|y|^{\frac{1}{2} l}\right)} .
$$

where we have the estimates (6.21) and with $n=2, \kappa=-\frac{1}{2^{m}}+1$ and $\varepsilon=\frac{1}{2^{m}}$ we obtain (6.30) and (6.31). 


\section{Bibliography}

[1] Berg, C., Sur les semi-groupes de convolution. Lecture Notes in Mathematics 404, 1-26, Springer, Berlin 1974.

[2] Berg, C., and Forst, G., Potential theory on locally compact Abelian groups. Springer Verlag, Berlin 1975.

[3] Beurling, A., and Deny, J., Espaces de Dirichlet. I. Le cas élémentaire. Acta Math. 99 (1958), 203-224.

[4] Beurling, A., and Deny, J., Dirichlet spaces, Proc. Natl. Acad. Sci. U.S.A. 45 (1959), 208-215.

[5] Bochner, S., Monotone Funktionen, Stieltjessche Integrale and harmonische Analyse. Math. Ann. 108 (1933), 378-410.

[6] Davies, E.B., Heat kernels and spectral theory. Cambridge Tracts in Mathematics, Vol. 92, Cambridge University Press, Cambridge 1989.

[7] Ethier, S.N., and Kurtz, T.G., Markov processes-characterization and convergence, Wiley Series in Probabability and Mathematical Statistics, John Wiley \& Sons, New York 1986.

[8] Evans, K., Fry, H., and Jacob, N., Fundamental Solutions in $\psi$-Bessel Potential Spaces of Certain Generators of Transient Lévy Processes.

[9] Farkas, W., Jacob, N., and Schilling, R.L., Function spaces related to continuous negative definite functions: $\psi$-Bessel potential spaces. Dissertationes Mathematicae 2001, 393, 1-62. 
[10] Friedlander, F.G., and Joshi, M., Introduction to the theory of distributions. 2nd edition. Cambridge University Press, Cambridge, 1998.

[11] Fukushima, M., Oshima, Y., and Takeda, M., Dirichlet forms and symmetric Markov processes. 2nd edition. de Gruyter Studies in Mathematics, Vol. 19. Walter de Gruyter Verlag, Berlin, 2011.

[12] Gradshteyn, I.S., and I.M. Ryzhik, Table of integrals, series, and products. Corrected and enlarged edition. Academic Press, San Diego 1980.

[13] Heinonen, J., Lectures on analysis on metric space. Springer-Verlag, New York 2001.

[14] Hirsch, F., Quotients de fonctions définies-négatives et synthèse spectrale. Ann. Inst. Fourier (Grenoble) 30.4 (1980), 75-96.

[15] Hoh, W. A symbolic calculus for pseudo differential operators generating Feller semigroups. Osaka J. Math, 35 (1998), 798-820.

[16] Hörmander, L., On the theory of general partial differential operators. Acta Math. 94, (1955), 161-248.

[17] Hörmander, L., The analysis of linear partial differential operators I. Grundlehren der mathematischen Wissenschaften, Vol. 256, Springer Verlag, Berlin 1983.

[18] Hörmander, L., The analysis of linear partial differential operators II. Grundlehren der mathematischen Wissenschaften, Vol. 257, Springer Verlag, Berlin 1983.

[19] Jacob, N., Dirichlet forms and pseudo differential operators. Exop. Math. 6 (1988), 313-351.

[20] Jacob, N., Pseudo-differential operators and Markov processes. Vol. I: Fourier analysis and semigroups. Imperial College Press, London 2001.

[21] Jacob, N., Pseudo-differential operators and Markov processes. Vol. II: Generators and their potential theory. Imperial College Press, London 2002. 
[22] Jacob, N., and Evans, K.P., A Course in analysis. Vol. III. Fourier analysis, ordinary differential equations, calculus of variations. World Scientific. 2017

[23] Jacob, N., Knopova, V., Landwehr, and S., Schilling, R.L., A geometric interpretation of the transition density of a symmetric Lévy process. Sci. China Math. 55, (2012), 1099-1126.

[24] Jacob, N., and Rhind, E.O.T., Aspects of Micro-local Analysis and Geometry in the Study of Lévy-type Generators. In: Open Quantum Systems. A Mathematical Perspective (eds. D.Bahns, A.Pohl, I.Witt). Birkhäuser Verlag, Basel 2019, 77-140.

[25] Jacob, N., and Schilling R.L., An analytic proof of the Lévy-Khinchin formula on $\mathbb{R}^{n}$. Publ. Math. Debrecen 53 (1998), 69-89.

[26] Kato, T., Perturbation theory for linear operators. Grundlehren der mathematischen Wissenshaften Vol. 132, Springer Verlag, Berlin 1966

[27] Knopova, V., and Schilling, R.L., A note on the existence of transition probability densities for Lévy processes. Forum Math. 25 (2013), 125-149.

[28] Landkof, N.S., Foundations of modern potential theory. Grundlehren der mathematischen Wissenschaften, Vol. 180, Springer Verlag, Berlin 1972.

[29] Landwehr S., On the geometry related to jump processes. PhD Thesis. Swansea: Swansea University, 2010.

[30] Ma, Z., and Röckner, M. Introduction to the theory of (non-symmetric) Dirichlet forms. Universitext, Springer Verlag, Berlin 1992.

[31] Mizuta, Y., Potential theory in Euclidean spaces. Gakuto International Series, Mathematical Sciences and Applications, Vol. 6, Gakkōtosho, Tokyo 1996.

[32] Pazy, A., Semigroups of linear operators and applications to partial differential equations. Applied Mathematical Sciences, Vol. 44, Springer Verlag, New York 1983 
[33] Reed, M., and Simon, B. Methods of modern mathematical physics. Vol. I. Functional analysis. Academic Press, New York 1972.

[34] Reed, M., and Simon, B. Methods of modern mathematical physics. Vol. II. Fourier analysis, self adjointness. Academic Press, New York 1975.

[35] Schilling, R.L., Song, R., and Vondracek, Z., Bernstein functions. Theory and applications. Walter de Gruyter. Berlin 2010.

[36] Schoenberg, I.J., Metric spaces and positive definite functions. Trans. Amer. Math. Soc. 44 (1938), 522-536.

[37] Stein, E.M., Singular integrals and differetiability properties of functions. Princeton Mathetmatical Series, Vol. 30, Princeton University Press, Princeton NJ 1970.

[38] Tanabe, H., Equations of evolution. Monographs and Studies in Mathematics, Vol. 6, Pitman Publishing, London 1979.

[39] Triebel, H., Interpolation theory, function spaces, differential operators. North Holland Mathematical Library, Vol. 18, North-Holland Publishing Company, Amsterdam 1978.

[40] Varopoulos, N. Th., Hardy-Littlewood theory for semigroups. J. Funct. Anal. 63 (1985), 240-260.

[41] Varopoulos, N. Th., Saloff-Coste, L., and Coulhon, T., Analysis and geometry on groups. Cambridge Tracts in Mathematics, Vol. 100, Cambridge University Press. Cambridge 1992. 